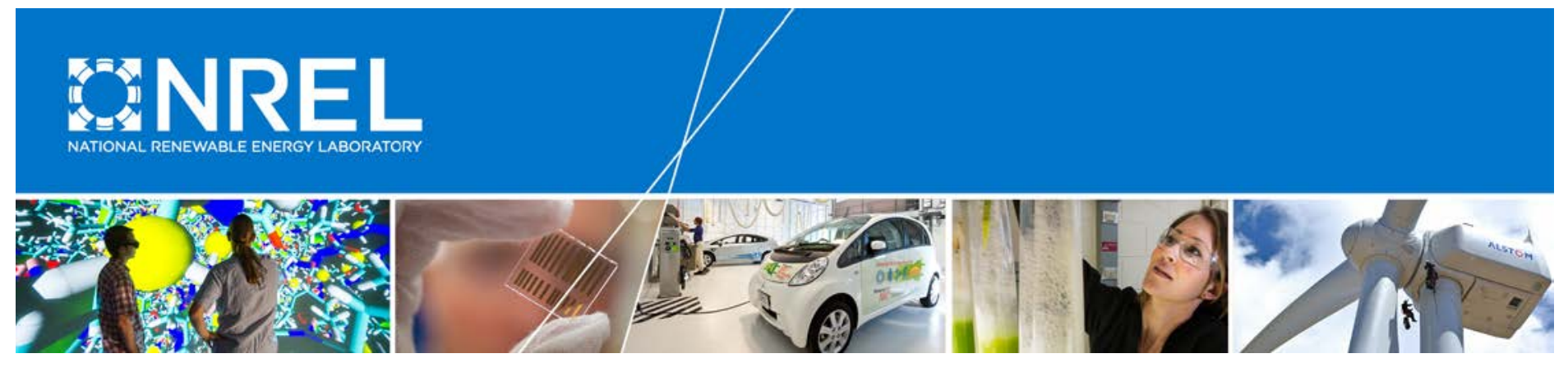

\title{
Best Practices Handbook for the Collection and Use of Solar Resource Data for Solar Energy Applications: Second Edition
}

Edited by Manajit Sengupta, ${ }^{1}$ Aron Habte, ${ }^{1}$ Christian Gueymard, ${ }^{2}$ Stefan Wilbert, ${ }^{3}$ and Dave Renné ${ }^{4}$

${ }_{2}^{1}$ National Renewable Energy Laboratory

${ }^{2}$ Solar Consulting Services

${ }^{3}$ German Aerospace Center (DLR)

${ }^{4}$ Dave Renné Renewables, LLC

This update was prepared in collaboration with the International Energy Agency Solar Heating and Cooling Programme: Task 46

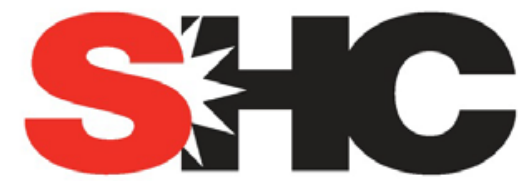

SOLAR HEATING \& COOLING PROGRAMME INTERNATIONAL ENERGY AGENCY

NREL is a national laboratory of the U.S. Department of Energy Office of Energy Efficiency \& Renewable Energy Operated by the Alliance for Sustainable Energy, LLC

This report is available at no cost from the National Renewable Energy Laboratory (NREL) at www.nrel.gov/publications.

Technical Report

NREL/TP-5D00-68886

December 2017

Contract No. DE-AC36-08GO28308 


\section{Best Practices Handbook for the Collection and Use of Solar Resource Data for Solar Energy Applications: Second Edition}

Manajit Sengupta, ${ }^{1}$ Aron Habte, ${ }^{1}$ Christian Gueymard, ${ }^{2}$ Stefan Wilbert, ${ }^{3}$ and Dave Renné ${ }^{4}$

${ }^{1}$ National Renewable Energy Laboratory

${ }^{2}$ Solar Consulting Services

${ }^{3}$ German Aerospace Center (DLR)

${ }^{4}$ Dave Renné Renewables, LLC

Prepared under Task No. SETP.10304.28.01.10
NREL is a national laboratory of the U.S. Department of Energy Office of Energy Efficiency \& Renewable Energy Operated by the Alliance for Sustainable Energy, LLC

This report is available at no cost from the National Renewable Energy Laboratory (NREL) at www.nrel.gov/publications.
National Renewable Energy Laboratory 15013 Denver West Parkway Golden, CO 80401

303-275-3000 • www.nrel.gov

\section{Technical Report}

NREL/TP-5D00-68886

December 2017

Contract No. DE-AC36-08GO28308 


\title{
NOTICE
}

This report was prepared as an account of work sponsored by an agency of the United States government. Neither the United States government nor any agency thereof, nor any of their employees, makes any warranty, express or implied, or assumes any legal liability or responsibility for the accuracy, completeness, or usefulness of any information, apparatus, product, or process disclosed, or represents that its use would not infringe privately owned rights. Reference herein to any specific commercial product, process, or service by trade name, trademark, manufacturer, or otherwise does not necessarily constitute or imply its endorsement, recommendation, or favoring by the United States government or any agency thereof. The views and opinions of authors expressed herein do not necessarily state or reflect those of the United States government or any agency thereof.

This report is available at no cost from the National Renewable Energy Laboratory (NREL) at www.nrel.gov/publications.

Available electronically at SciTech Connect http:/www.osti.gov/scitech

Available for a processing fee to U.S. Department of Energy and its contractors, in paper, from:

\author{
U.S. Department of Energy \\ Office of Scientific and Technical Information \\ P.O. Box 62 \\ Oak Ridge, TN 37831-0062 \\ OSTI http://www.osti.gov \\ Phone: 865.576.8401 \\ Fax: 865.576.5728 \\ Email: reports@osti.gov
}

Available for sale to the public, in paper, from:

\author{
U.S. Department of Commerce \\ National Technical Information Service \\ 5301 Shawnee Road \\ Alexandria, VA 22312 \\ NTIS http://www.ntis.gov \\ Phone: 800.553 .6847 or 703.605 .6000 \\ Fax: 703.605.6900 \\ Email: orders@ntis.gov
}




\section{Foreword for the Handbook}

Designing, financing, and operating successful solar heating, concentrating solar power, and photovoltaic systems requires reliable information about the solar resource available and its variability over time. In the past, seasonal and daily variability has been studied and understood; however, with new solar technologies becoming more important in energy supply grids, small time-scale effects are critical to successful deployment of these important low carbon technologies. A vital part of the bankability of solar projects is to understand the variability of the solar resource so that supply and storage technologies can be optimized.

This handbook is the result of 10 years of international collaboration carried out by experts from the International Energy Agency's (IEA's) Solar Heating and Cooling (SHC), Solar PACES, and Photovoltaic Power Systems Technology Collaboration Programmes. Under IEA SHC Task 46: Solar Resource Assessment and Forecasting, experts from 11 countries produced information products and best practices on solar energy resources that will greatly benefit project developers and system operators as well as assist policymakers in advancing renewable energy programs worldwide.

Meteorologists, mathematicians, solar technology specialists, and other key solar resource experts from around the world joined forces to further our understanding of the sun's temporal and spatial variability through benchmarking satellite-derived solar resource data and solar forecasts, developing best practices for measuring the solar resource, and conducting research to improve satellite-based algorithms. The results of IEA SHC Task 46 are useful to a wide range of users of solar heating and cooling, photovoltaics, and concentrating solar power systems and of building developers and owners as well as anyone else who needs to understand and predict sunlight for agricultural or other purposes.

The earlier edition of the handbook, which was published in 2015 , is used worldwide as a reference for each stage of a solar energy project. Since that time, there has been substantial growth in the interest in high-quality "bankable" solar resource data. This revision adds significant new methods so it will be even more useful.

This publication is a summary that details the fundamentals of solar resources as well as captures the state of the art. For those wanting more depth, it also provides the references where more detailed information can be found.

I would like to acknowledge the leadership of the National Renewable Energy Laboratory and express appreciation to the U.S. Department of Energy for producing the handbook and incorporating results from IEA SHC Task 46.

Ken Guthrie

Chair, IEA Solar Heating and Cooling Technology Collaboration Programme June 2017 


\section{Preface}

\section{Dave Renné}

Dave Renné Renewables, $L L C$

As the world looks for carbon-free sources to meet final energy demand associated with heat, electrical power, and transport, energy from the sun stands out as the single most abundant energy resource on earth. Harnessing this energy is the challenge and opportunity for achieving a carbon-free energy supply by the end of this century. Photovoltaics, solar heating and cooling, and concentrating solar power (CSP) are the primary forms of energy applications using sunlight. These solar energy systems use different technologies, collect different fractions of the solar resource, and have different siting requirements and production capabilities. Reliable information about the solar resource is required for every solar energy application. This holds true for small installations on a rooftop as well as for large solar power plants. However, solar resource information is of particular interest for large installations because it requires a substantial investment, sometimes exceeding $\$ 1$ billion in construction costs. Before such a project is undertaken, the best possible information about the quality and reliability of the fuel source must be made available. That is, project developers need to have reliable data about the solar resource available at specific locations, including historic trends with seasonal, daily, hourly, and (preferably) sub-hourly variability to predict the daily and annual performance of a proposed power plant. Without these data, an accurate financial analysis is not possible.

In response to a meeting of prominent CSP developers and stakeholders hosted by the U.S. Department of Energy (DOE) in September 2008, the National Renewable Energy Laboratory (NREL) produced the first edition of a handbook to provide best practices for the use of solar resource data, which was titled Concentrating Solar Power: Best Practices Handbook for the Collection and Use of Solar Resource Datal ${ }^{1}$. The content was based on the experiences of scientists and engineers from industry, academia, and DOE for identifying the sources, quality, and methods for applying solar and meteorological data to CSP projects. During this same time period, the International Energy Agency's (IEA's) Solar Heating and Cooling Programme (SHC) was hosting a task on solar resource knowledge management (Task 36, which ran from 2005-2011) and then a task on solar resource assessment and forecasting (Task 46, which ran from 20112016). These tasks brought together the world's foremost experts in solar energy meteorology. This group of experts felt the need to create a collective document to disseminate the knowledge that was being developed through these tasks. It was decided that combining the efforts of the experts involved in the IEA tasks to build on the information in NREL's first version of the handbook would provide the best use of resources and deliver a handbook of outstanding quality to users. It was also decided that additional solar technologies, such as photovoltaics, would be incorporated along with additional aspects of energy meteorology that have become extremely important, such as solar forecasting. As a result, a second edition of the handbook, titled Best Practices

\footnotetext{
${ }^{1}$ https://www.nrel.gov/docs/fy10osti/47465.pdf
} 
Handbook for the Collection and Use of Solar Resource Data for Solar Energy Applications, was published by NREL in 2015. This served as an interim deliverable for Task 46, with the intention of producing a second edition as a final deliverable for the task after the task's conclusion at the end of 2016. The second edition of the handbook is presented here.

The solar energy industry has developed rapidly throughout the last few years, and there have been significant enhancements in the body of knowledge in the areas of solar resource assessment and forecasting. Thus, this second edition of the handbook updates and enhances the initial versions and presents the state of the art in a condensed form for all of its users. Chapter 1 lays out the need for high-quality and reliable solar resource data to support the rapidly growing industry, and Chapter 2, as with the previous editions, provides a basic tutorial on solar resources. Chapter 3 presents a comprehensive overview of best practices for measuring solar resources, including information gained under collaborative work completed under Task 46, and Chapter 4 summarizes techniques used to develop estimates of solar resources from weather satellite data and numerical model predictions. Chapter 5 lists a number of commonly used data sets available both in the public and private sectors (updated from the previous editions), and Chapter 6 provides important information on both measured and modeled solar data uncertainty. Chapter 7 provides an update on recent developments in the ability to forecast solar resources over time horizons spanning nowcasting to hours-ahead and days-ahead. All of this information leads up to Chapter 8 , which provides data application techniques for the various stages of project development, from pre-feasibility to routine operations, as shown in Figure P-1. The outlook for future work is summarized in Chapter 9.

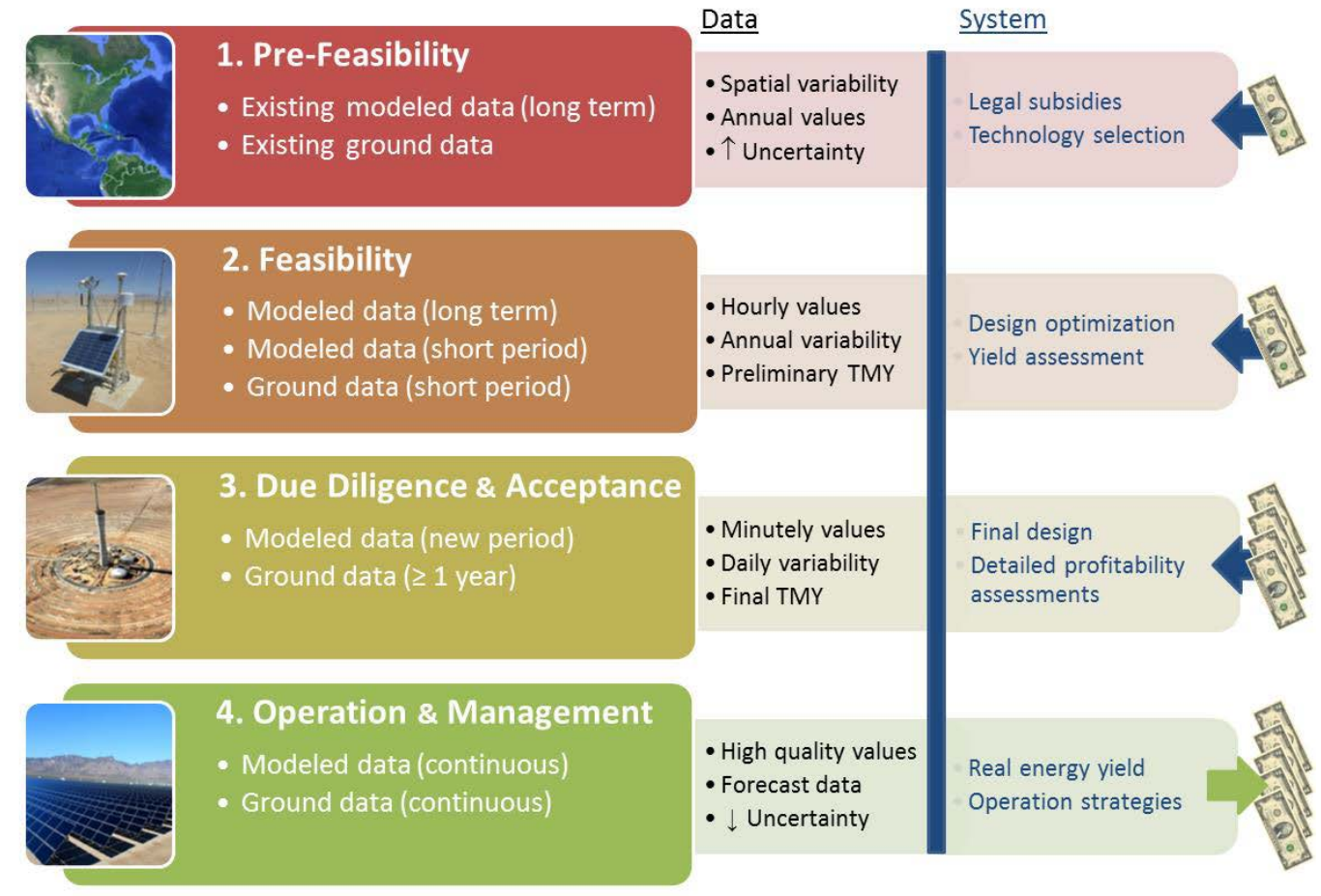

Figure P-1. Data application techniques for the various stages of project development 
Project developers, engineering procurement construction firms, utility companies, system operators, energy suppliers, financial investors, organizations involved in planning and managing solar research programs, and others involved in solar energy systems planning and development will find this handbook to be a valuable resource for the collection and interpretation of solar resource data. Readers are encouraged to provide feedback to the authors for future revisions and an expansion of the handbook's scope and content. 


\section{Acknowledgements}

This work was supported by the U.S. Department of Energy under Contract No. DE-AC36-08GO28308 with the National Renewable Energy Laboratory (NREL). The authors acknowledge the Solar Energy Technologies Office for supporting this research. Specifically, the authors are grateful to Dr. Anastasios Golnas, technology manager for the Systems Integration team of DOE, Office of Energy Efficiency and Renewable Energy, Solar Energy Technologies Office, for his support and encouragement.

The authors also thank the Solar Heating and Cooling Programme of the International Energy Agency for facilitating Task 46, without which this publication would not have been possible.

The authors also thank NREL Communications for their tireless work during the publication process.

Finally, special thanks to the following experts who spent hours of their valuable time reviewing the chapters and making excellent suggestions that significantly improved the quality of this handbook:

- Stephen Wilcox, Solar Resource Solutions, LLC

- Hans Georg Beyer, University of the Faroe Islands

- Thomas Stoffel, Solar Resource Solutions, LLC

- Marion Schroedter-Homscheidt, German Aerospace Center (DLR)

- Lucien Wald, MINES ParisTech

- Jan Remund, Meteotest

- Mike Dooraghi, National Renewable Energy Laboratory

- Detlev Heinemann, Oldenburg University

- Jan Kleissl, University of California, San Diego. 


\section{List of Acronyms}

3D

$\mathrm{ABI}$

$\mathrm{AC}$

ACR

AERONET

AHF

AHI

AM

AM1

AM2

AOD

APOLLO

ARM

Ås

ASTM

AU

AVHRR

BIPM

BSRN

CAMS

$\mathrm{C}_{\text {cal }}$

CDF

CERES

CFSR

CI

CIEMAT

CIMO

CMV

$\mathrm{COV}$

$\mathrm{CPC}$

CPV

CSP

CST

DC

DEM

DHI

DLR

DNI

DOE

DRY

ECMWF three-dimensional

Advanced Baseline Imager

alternating current

active cavity radiometer

Aerosol Robotic Network

Automatic Hickey-Frieden

Advanced Himawari Imager

air mass

air mass one

air mass two

aerosol optical depth

AVHRR processing scheme over clouds, land, and ocean

Atmospheric Radiation Measurement

Ångström Scale

American Society for Testing and Materials

astronomical unit

Advanced Very High Resolution Radiometer

International Bureau of Weights and Measures

Baseline Surface Radiation Network

Copernicus Atmospheric Monitoring Service

Calibration Factor

cumulative distribution function

Cloud and Earth's Radiant Energy System

Climate Forecast System Reanalysis

confidence interval

Centro de Investigaciones Energéticas,

Medioambientales y Tecnológicas

Commission for Instruments and Methods of

Observation

cloud motion vector

coefficient of variation

compound parabolic collector

concentrating photovoltaic

concentrating solar power

concentrating solar technologies

direct current

digital elevation model

diffuse horizontal irradiance

German Aerospace Center

direct normal irradiance

U.S. Department of Energy

design reference years

European Center for Medium-Range Weather

Forecasts 


$\begin{array}{ll}\text { ED } & \text { electrodialysis } \\ \text { ERY } & \text { energy reference years } \\ \text { ETR } & \text { extraterrestrial radiation } \\ \text { ETS } & \text { extraterrestrial spectrum } \\ \text { EUMETSAT } & \text { European Organization for the Exploitation of } \\ & \text { Meteorological Satellites Union } \\ \text { FARMS } & \text { Fast All-sky Radiation Model for Solar } \\ & \text { Applications } \\ \text { FLASHFlux } & \text { Fast Longwave and Shortwave Radiative Fluxes } \\ \text { FOV } & \text { field of view } \\ \text { GEM } & \text { Global Environmental Multiscale } \\ \text { GFS } & \text { Global Forecast System } \\ \text { GHI } & \text { global horizontal irradiance } \\ \text { GIS } & \text { geographic information system } \\ \text { GOES } & \text { Geostationary Operational Environmental Satellite } \\ \text { GTI } & \text { global tilted irradiance } \\ \text { GUM } & \text { The Guide to the Expression of Uncertainty in } \\ \text { HIRLAM } & \text { Measurements } \\ \text { IAU } & \text { High-Resolution Limited Area Model } \\ \text { IDMP } & \text { International Astronomical Union } \\ \text { IEA } & \text { International Daylight Measurement Program } \\ \text { IEC } & \text { International Energy Agency } \\ \text { IFS } & \text { International Electro-technical Commission } \\ \text { IPCC } & \text { Integrated Forecast System } \\ \text { IPS } & \text { International Panel of Climate Change } \\ \text { IrSoLaV } & \text { International Pyrheliometric Scale } \\ \text { ISCCP } & \\ \text { ISIS } & \text { International Satellite Cloud Climatology Project } \\ \text { ISO } & \text { Integrated Surface Irradiance Study } \\ \text { JPSS } & \text { International Organization for Standardization } \\ \text { Kd } & \text { Joint Polar Satellite System } \\ \text { Kn } & \text { Clearness index or Diffuse horizontal transmittance } \\ \text { KS } & \text { Clearness index or Direct normal transmittance } \\ \text { KSI } & \text { Kolmogorov-Smirnov } \\ \text { Kt } & \text { Kolmogorov-Smirnov integral } \\ \text { LAT } & \text { Clearness index or global horizontal transmittance } \\ \text { LCOE } & \text { local apparent time } \\ \text { LES } & \text { levelized cost of electricity } \\ \text { LiDAR } & \text { Large Eddy Simulation } \\ \text { LRS } & \text { light detection and ranging } \\ & \text { land constrains, radiation, and slope potential } \\ \text { LST } & \text { assessment model } \\ \text { MACC } & \text { local standard time } \\ \text { MACC-RAD } & \text { Monitoring Atmospheric Composition and Climate } \\ & \text { Monitoring Atmospheric Composition and Climate } \\ & \text { - Radiation } \\ & \end{array}$




MADCast
MAE
MBE
McICA
MCP
MED
MENA
MERRA-2
MESoR
METEOSAT
METSTAT
MFG
MISR
MM5
MMDT
MODIS
MOS
MSE
MSF
MSG
MTG
MTSAT
NASA
NCAR
NESDIS
NIP
NOAA
NPV
NREL
NSRDB
NWP
O\&M
OMPS
P
PATMOS-x
PDF
POA
POE
PR
PSA
PSM
PSP
PV
PVGIS
M

Multisensor Advection Diffusion nowCast mean absolute error mean bias error Monte Carlo Independent Column Approximation measure-correlate-predict Multi Effect Distillation Middle East and North Africa region Modern Era Retrospective analysis for Research and Applications, Version 2 Management and Exploitation of Solar Resource Knowledge

METeorolgoical STATistical METEOSAT first generation Multi-angle Imaging SpectroRadiometer mesoscale model of fifth generation monthly means of daily totals Moderate Resolution Imaging Spectroradiometer model output statistic mean square error Multi Stage Flash distillation METEOSAT second generation METEOSAT third generation Multifunction Transport Satellite National Aeronautics and Space Administration National Center for Atmospheric Research NOAA's Satellite and Information Service normal incidence pyrheliometer National Oceanic and Atmospheric Administration net present value

National Renewable Energy Laboratory National Solar Radiation Database numerical weather prediction operations and maintenance Ozone Mapping and Profile Suite percentile

Pathfinder Atmospheres Extended probability density function plane of array probability of exceedance performance ratio

Plataforma Solar de Almería Physical Solar Model Precision Spectral Pyranometer photovoltaic photovoltaic geographical information system 


$\begin{array}{ll}\text { RBR } & \text { red-to-blue } \\ \text { REST2 } & \text { Reference Evaluation of Solar Transmittance, } 2 \\ & \text { bands } \\ \text { RMSD } & \text { root mean square deviation } \\ \text { RMSE } & \text { root mean square error } \\ \text { RO } & \text { reverse osmosis } \\ \text { RReDC } & \text { Renewable Resource Data Center } \\ \text { RRTM } & \text { Rapid Radiation Transfer Model } \\ \text { RRTMG } & \text { Rapid Radiation Transfer Model for General } \\ & \text { Circulation Models } \\ \text { Rs } & \text { Responsivity } \\ \text { RSI } & \text { rotating shadowband irradiometer } \\ \text { RSP } & \text { rotating shadowband pyranometer } \\ \text { RSR } & \text { rotating shadowband radiometer } \\ \text { RSY } & \text { representative solar year } \\ \text { SAM } & \text { System Advisory Model } \\ \text { SC } & \text { solar constant } \\ \text { SCAPP } & \text { Scanning Pyrheliometer/Pyranometer } \\ \text { SHC } & \text { Solar Heating and Cooling Programme } \\ \text { Si } & \text { silicon } \\ \text { SI } & \text { Système International } \\ \text { SO } & \text { sulfur dioxide } \\ \text { SODA } & \text { Solar radiation data } \\ \text { SOLEMI } & \text { Solar Energy Mining } \\ \text { SOLMET } & \text { Solar and Meteorological } \\ \text { SRB } & \text { Surface Radiation Budget } \\ \text { SS } & \text { Smithsonian Scale } \\ \text { SSE } & \text { surface meteorology and solar energy } \\ \text { STC } & \text { Standard Test Condition } \\ \text { STE } & \text { solar thermal electricity } \\ \text { SUNY } & \text { States University of New York } \\ \text { Suomi NPP } & \text { Suomi National-Polar-Orbiting Partnership } \\ \text { SURFRAD } & \text { Surface Radiation Budget Network } \\ \text { SWERA } & \text { Solar and Wind Energy Resource Assessment } \\ \text { SZA } & \text { solar zenith angle } \\ \text { TDY } & \text { typical direct normal irradiance year } \\ \text { TGY } & \text { typical global horizontal irradiance year } \\ \text { TMM } & \text { typical meteorological month } \\ \text { TMY } & \text { typical meteorological year } \\ \text { TOA } & \text { top of atmosphere } \\ \text { TRY } & \text { typical reference year } \\ \text { TSI } & \text { total solar irradiance } \\ \text { TSR } & \text { Thermopile Shadowband Radiometer } \\ \text { UPS } & \text { uninterruptible power supply } \\ \text { UT } & \text { Universal Time } \\ \text { UTC } & \text { Coordinated Universal Time } \\ & \\ & \end{array}$


UV

VIIRS

WCRP

WMO

WRC

WRDC

WRF

WRR ultraviolet

Visible Infrared Imaging Radiometer Suite

World Climate Research Programme

World Meteorological Organization

World Radiation Center

World Radiation Data Center

Weather Research and Forecasting

World Radiometric Reference 


\section{Table of Contents}

Chapter 1: Why Solar Resource Data Are Important to Solar Power ................................................... 1-1

Chapter 2: Overview of Solar Radiation Resource Concepts ............................................................ 2-1

Chapter 3: Measuring Solar Radiation and Relevant Atmospheric Parameters................................... 3-1

Chapter 4: Modeling Solar Radiation-Current Practices ................................................................ 4-1

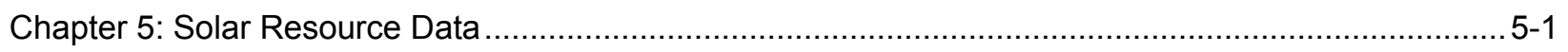

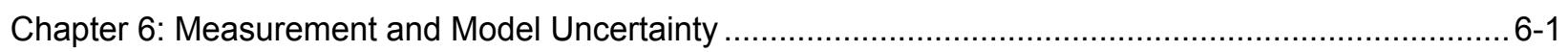

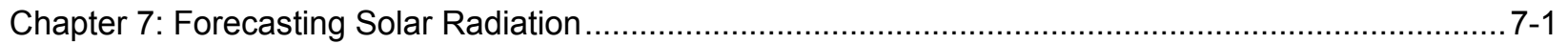

Chapter 8: Applying Solar Resource Data to Solar Energy Projects .................................................... 8-1

Chapter 9: Future Work............................................................................................................ 9-1 


\title{
1 Why Solar Resource Data Are Important to Solar Power
}

\author{
Richard Meyer $^{1}$ and Manajit Sengupta ${ }^{2}$ \\ 1. Suntrace $\mathrm{GmbH}$ \\ 2. National Renewable Energy Laboratory
}

Sunlight is the fuel for all solar energy generation technologies. For any generation source, knowledge of the quality and future reliability of the fuel is essential for accurate analyses of system performance and to determine the financial viability of a project. For solar energy systems, the variability of the supply of sunlight probably represents the single greatest uncertainty in a solar power plant's predicted performance. Solar resource data and modeling factor into three elements of a solar project's life:

- Historical long-term data for site selection during feasibility studies

- Prediction of power plant output for plant design and financing

- Real-time measurement and solar forecasting for plant and grid operations.

Site selection includes numerous location properties, including current land use, grid access, and proximity to load centers, but a top priority is determining if an adequate solar resource exists for a proposed project. For site selection, average solar irradiation at the site is the first selection criterion. Geographical latitude is also considered because sites close to the equator have advantages such as lower geometrical losses and lower shadowing. Lower seasonal variability at locations near the equator could also be advantageous because of a more consistent match to the power demand. As weather patterns may change from year to year, many years of data are required for determining reliable average irradiation conditions and inter-annual variability. For this purpose, satellite-derived, high-quality historic solar radiation data sets covering over 10 years are usually considered necessary for site selection, although site-specific climate conditions or design criteria may allow a shorter period.

As flat-priced electricity feed-in-tariff regulations get phased out, the economic yield of solar power systems depends more and more on the solar production during various times of the year as well as on its availability during specific parts of the day. Thus, for solar projects with variable prices, the temporal distribution of solar irradiance may be critical, even during site selection, to estimate potential yields among alternative sites. At this early stage of project development, it is sufficient to study the temporal variability of the energy output throughout the year and typical daily cycles. As an alternative to multiple-year data sets, typical meteorological year (TMY) data for each site may be sufficient at this stage, although the TMY will not characterize inter-annual variability.

If a site is found to be feasible and a power project is to be developed, more precise and detailed data sets are required. For the site-specific techno-economic optimization of a solar system, availability of higher time resolution data is always beneficial. Advanced modeling techniques allow developing such data based on satellite-derived time series. For financing large solar plants, data sets that are validated by ground measurements on or near the site become essential to lower the yield risk. In addition to precise solar radiation measurements, specialized 
meteorological stations usually provide additional environmental parameters that help to optimize the sizing and proper selection of plant components.

Precise solar and meteorological stations are also valuable during plant commissioning; reliable measurements are the base for acceptance testing to demonstrate proof of fulfillment of technical specifications for heat or electric output. Although temporary measurement equipment may be used for acceptance testing, reliable measurements are essential for estimating real-time plant output to assure high efficiency of the plant throughout its service life. Evaluation of plant output as a function of solar irradiance is the most important indicator of power plant performance. A drop in overall efficiency implies a degradation of one or more power plant components or poor maintenance or operation. Although remotely sensed data may be used for smaller rooftop systems where performance accuracy can be relaxed, larger solar systems usually rely on ground-based measurements, which may be combined with near-real-time satellite-derived solar radiation data. Local ground measurements also assist in site-specific model validation and improvements of solar forecasting.

Proper and accurate solar forecasts are important for ideal use of solar plants both economically and operationally. They help to improve system operations such as optimal use of a storage tank in a solar thermal water heating system, a molten salt system for high-temperature applications, or a battery system in an off-grid photovoltaic (PV) system. With the fast growth of gridconnected solar electrical systems, solar radiation forecasts have become highly important for safe grid operations and efficient operations among power plants, which might be necessary to balance solar fluctuations.

This handbook covers all pertinent aspects of solar radiation, which are relevant for the planning and operation of solar thermal heating and cooling systems, as well as for concentrating solar thermal and PV plants. Chapter 2 explains the basic concepts and terms, which are essential for understanding subsequent chapters. Chapter 3 describes the state of the art in measuring solar radiation on the Earth's surface and offers methods and protocols to produce a data set that withstands the scrutiny of due diligence. Chapter 4 focuses on modeling solar radiation. It provides an introduction to the theory of radiative transfer in the atmosphere, aiming to provide an understanding of current practices for deriving solar radiation values at the Earth's surface. Several examples of solar resource data sets derived from satellites and ground-measured data are presented in Chapter 5. It is important to understand the uncertainty of any data set produced by either measurement or modeling. Chapter 6 provides an understanding of how to estimate and interpret uncertainty in both measured and modeled data sets. Today, many data sets are retrieved from operational meteorological satellites applying radiative theory. Radiative transfer calculations, similar to those described in Chapter 4, are used to forecast the intensity of solar radiation, which is described in Chapter 7. Nowcasting a few hours ahead by extrapolating satellite and ground-mounted observations is now state of the art, while solar radiation forecasts beyond the first few hours are estimated by numerical weather prediction models. Chapter 8 summarizes the various techniques and data sets and recommends best practices for the various stages of a solar power project. Significant work remains to improve the accuracy, reliability, and level of detail of solar resource products. It is recognized that many open questions remain in the field of solar resource assessment. Chapter 9 gives an overview of how these outstanding issues may be solved in the future through the development of new or improved techniques and 
applying new measurement techniques, new meteorological satellites, improved weather models, or, ideally, a smart combination of these approaches. 


\title{
2 Overview of Solar Radiation Resource Concepts
}

\author{
Aron Habte, ${ }^{1}$ Thomas Stoffel, ${ }^{2}$ Richard Perez, ${ }^{3}$ Daryl Myers, ${ }^{4}$ Christian Gueymard, ${ }^{5}$ \\ Philippe Blanc, ${ }^{6}$ and Stefan Wilbert ${ }^{7}$ \\ 1. National Renewable Energy Laboratory \\ 2. Solar Resource Solutions, $L L C$ \\ 3. State University of New York, ASRC \\ 4. Retired, National Renewable Energy Laboratory \\ 5. Solar Consulting Services \\ 6. German Aerospace Center (DLR)
}

\subsection{Introduction}

Describing the relevant concepts and applying a consistent terminology are important to the usefulness of any handbook. This chapter uses a standard palette of terms to provide an overview of the key characteristics of solar radiation - the fuel for all solar technologies.

Beginning with the sun as the source, we present an overview of the effects of Earth's orbit and atmosphere on the possible types and magnitudes of solar radiation available for energy conversion. An introduction to the concepts of measuring and modeling solar radiation is intended to prepare the reader for the more in-depth treatments in Chapter 3-5. This overview concludes with an important discussion of the estimated uncertainties associated with solar resource data, as affected by the experimental and modeling methods used to produce the data. The reader is referred to Chapter 6 for a detailed treatment of uncertainty in measurement and modeling.

\subsection{Radiometric Terminology}

Before continuing our discussion of solar radiation, it is important to understand basic radiometric terms. Radiant energy, flux, power, and other concepts used in this handbook are summarized in Table 2-1.

Table 2-1. Radiometric Terminology and Units

\begin{tabular}{|l|l|l|l|l|}
\hline \multicolumn{1}{|c|}{ Quantity } & Symbol & \multicolumn{1}{|c|}{ SI Unit } & Abbreviation & Description \\
\hline $\begin{array}{l}\text { Radiant } \\
\text { energy }\end{array}$ & Q & Joule & $\mathrm{J}$ & Energy \\
\hline $\begin{array}{l}\text { Radiant } \\
\text { flux }\end{array}$ & $\Phi$ & Watt & $\mathrm{W}$ & $\begin{array}{l}\text { Radiant energy per unit of time } \\
\text { (radiant power) }\end{array}$ \\
\hline $\begin{array}{l}\text { Radiant } \\
\text { intensity }\end{array}$ & $\mathrm{I}$ & Watt per steradian & $\mathrm{W} / \mathrm{sr}$ & Power per unit solid angle \\
\hline $\begin{array}{l}\text { Radiant } \\
\text { emittance }\end{array}$ & $\mathrm{M}$ & $\begin{array}{l}\text { Watt per square } \\
\text { meter }\end{array}$ & $\mathrm{W} / \mathrm{m}^{2}$ & Power emitted from a surface \\
\hline Radiance & L & $\begin{array}{l}\text { Watt per steradian } \\
\text { per square meter }\end{array}$ & $\mathrm{W} /\left(\mathrm{m}^{2} \cdot \mathrm{sr}\right)$ & $\begin{array}{l}\text { Power per unit solid angle per unit of } \\
\text { projected source area }\end{array}$ \\
\hline Irradiance & $\mathrm{E}, \mathrm{I}$ & $\begin{array}{l}\text { Watt per square } \\
\text { meter }\end{array}$ & $\mathrm{W} / \mathrm{m}^{2}$ & Power incident on a unit area surface \\
\hline
\end{tabular}




\begin{tabular}{|l|l|l|l|l|}
\hline $\begin{array}{l}\text { Spectral } \\
\text { irradiance }\end{array}$ & $\mathrm{E}_{\lambda}$ & $\begin{array}{l}\text { Watt per square } \\
\text { meter per nanometer }\end{array}$ & $\mathrm{W} /\left(\mathrm{m}^{2} \cdot \mathrm{nm}\right)$ & $\begin{array}{l}\text { Power incident on a unit area surface per } \\
\text { unit wavelength measured in nanometers }\end{array}$ \\
\hline Irradiation & $\mathrm{H}$ & $\begin{array}{l}\text { Joule per square } \\
\text { meter }\end{array}$ & $\mathrm{J} / \mathrm{m}^{2}$ & $\begin{array}{l}\text { Energy accumulated on a unit area surface } \\
\text { over a period; a more practical energy unit is } \\
\text { kilowatt-hours }\left(1 \mathrm{kWh} / \mathrm{m}^{2}=3.6 \mathrm{MJ} / \mathrm{m}^{2}\right)\end{array}$ \\
\hline
\end{tabular}

\subsection{Extraterrestrial Irradiance}

Any object with a temperature above absolute zero emits radiation. With an effective surface temperature of $\approx 5,800 \mathrm{~K}$, the sun behaves like a quasi-static blackbody and emits radiation over a wide range of wavelengths, with a distribution that is close to what Planck's law predicts (Figure 2-1). This constitutes the solar spectral power distribution, or solar spectrum. For terrestrial applications, the useful solar spectrum, also called shortwave spectrum, includes the spectral regions called ultraviolet (UV), visible, and near-infrared from about 290-4,000 nm (Figure 2-1). In contrast, the longwave (or infrared) spectrum includes wavelengths ranging from about 4-50 $\mu \mathrm{m}$. Based on a recent determination (Gueymard 2004), most of the spectral irradiance $(98.5 \%)$ of the extraterrestrial spectrum (ETS) is contained in the wavelength range of 290-4,000 nm. In what follows, broadband solar radiation will always refer to this spectral range unless specified otherwise.

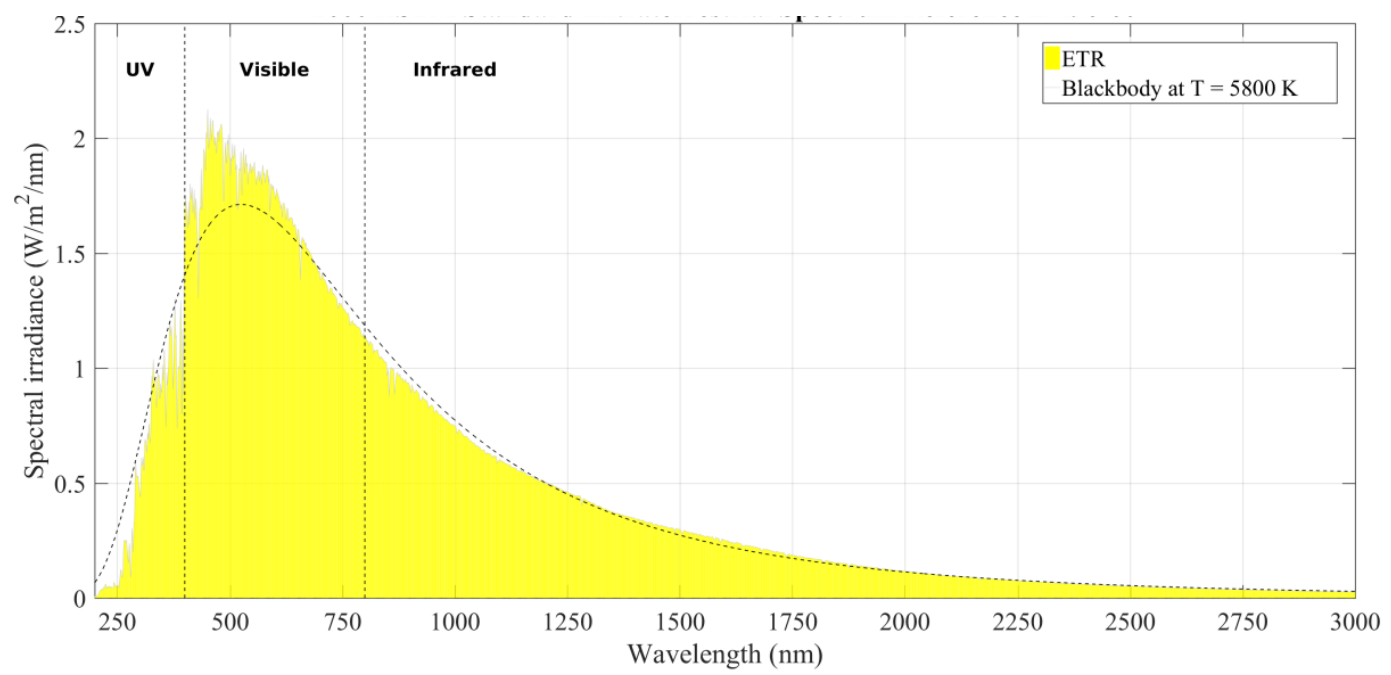

Figure 2-1. Reference extraterrestrial spectrum (ASTM E-490-06) and 5,800 K blackbody distribution using Planck's law. Image by Philippe Blanc, MINES-ParisTech / ARMINES.

Various ETS distributions have been derived based on ground measurements, extraterrestrial measurements, and physical models of the sun's output. Some historical perspective is offered by Gueymard (2004, 2006). All distributions contain some deviations from the current standard extraterrestrial spectra used by the American Society for Testing and Materials (ASTM) Standard E490 (2006) (cf. Figure 2-1). This standard was actually first published in 2000 and is based on older data than the 2004 ETS. Some of its shortcomings have been discussed (Gueymard 2004, 2006). A new generation of ETS distributions is now in preparation based on recent spectral measurements from space. 


\subsection{Solar Constant}

The total radiant power from the sun is nearly constant. In fact, the solar output (radiant emittance), which can be obtained as the integration of the ETS over all wavelengths, has commonly been called the solar constant (SC) until its short-term and long-term variations were identified and quantified. To account for this variability, the solar output at any moment is now referred to as total solar irradiance (TSI). There are cycles of about 11 years in solar activity, which are accompanied by a varying number of sunspots (cool, dark areas on the sun) and faculae (hot, bright spots). TSI increases during high-activity periods because the numerous faculae more than counterbalance the effect of sunspots. The concept of SC is still useful and can be considered as the long-term TSI average. Figure 2-2 shows the latest version of a composite TSI time series based on multiple space-borne instruments that have monitored TSI since 1978, using a variety of instruments and absolute scales. Estimates are also used for the period 19751978 to make this time series start at the onset of sun cycle 21. TSI is normalized to 1 astronomical unit (AU), the average Earth-sun distance. Figure 2-2 shows SC (always evaluated at $1 \mathrm{AU}$ ) as a horizontal solid green line. Its value is $\approx 1,366 \mathrm{~W} / \mathrm{m}^{2}$ according to the scale on the left $\mathrm{y}$-axis (old reference) and $\approx 1,361 \mathrm{~W} / \mathrm{m}^{2}$ according to that on the right y-axis (new reference).

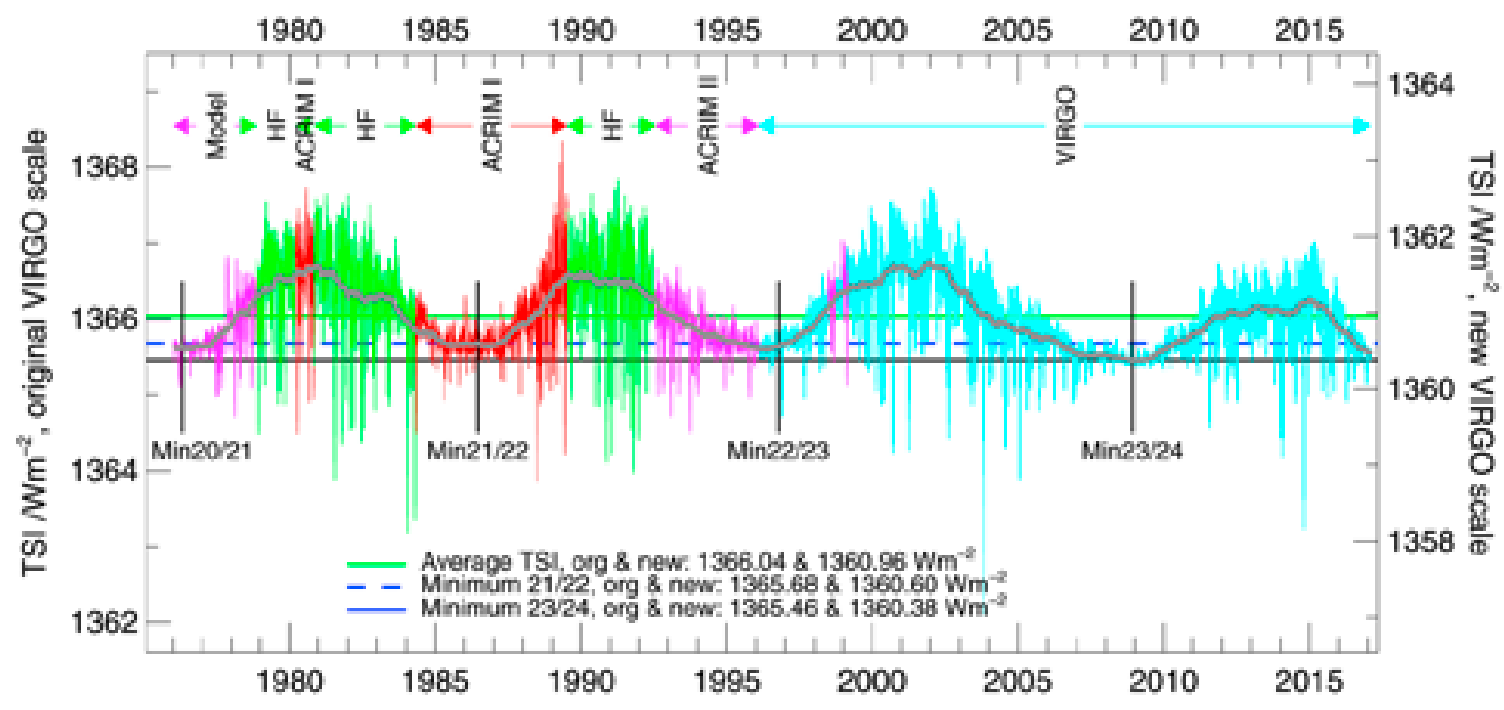

Figure 2-2. Four solar cycles show the temporal variations of TSI in composite measurements from satellite-based radiometers (color coded) and model results produced by the World Radiation Center (WRC). ${ }^{1}$ Image used with permission from the Physical Meteorological Observatory in Davos, Switzerland

On a daily basis, the passage of large sunspots results in much lower TSI values than the SC. The measured variation in TSI resulting from the sunspot cycle is at most $\pm 0.2 \%$, only twice the precision (i.e., repeatability - not total absolute accuracy, which is approximately $\pm 0.5 \%$ ) of the most accurate radiometers measuring TSI in space. There is, however, some large variability in a few spectral regions—especially the UV (wavelengths less than $400 \mathrm{~nm}$ ) —caused by solar activity.

\footnotetext{
${ }^{1}$ See http://www.pmodwrc.ch/pmod.php?topic=tsi/composite/SolarConstant.
} 
Historic determinations of SC have fluctuated over time (Gueymard 2006). At the onset of the $21^{\text {st }}$ century, it was considered to be $1,366.1 \pm 7 \mathrm{~W} / \mathrm{m}^{2}$ (ASTM 2000; Gueymard 2004).

However, more recent satellite observations using advanced sensors and better calibration methods have shown that SC was actually somewhat lower, $\approx 1,361 \mathrm{~W} / \mathrm{m}^{2}$. For instance, Gueymard (2012) proposed a revised value of 1,361.2 W/m². According to astronomical computations such as those made by the National Renewable Energy Laboratory's (NREL's) solar position software, ${ }^{2}$ using $\mathrm{SC} \approx 1,361 \mathrm{~W} / \mathrm{m}^{2}$, the variation in the Earth-sun distance causes the ETR to vary from $\approx 1,409 \mathrm{~W} / \mathrm{m}^{2}$ around January 3 to $\approx 1,315 \mathrm{~W} / \mathrm{m}^{2}$ around July 4 . The latter variation is systematic and deterministic. Hence, it does not include the daily (somewhat random) or cyclical variability in TSI induced by solar activity, which was discussed above. This variability being less than $\pm 0.2 \%$ is simply ignored in the practice of solar resource assessments.

\subsection{Solar Geometry}

The amount of radiation exchanged between two objects is affected by their separation distance. The Earth's elliptical orbit (eccentricity 0.0167 ) brings it closest to the sun in January and farthest from the sun in July. This annual variation results in a $\pm 3.4 \%$ difference in the Earth's solar irradiance. The average Earth-sun distance is close to the new definition of the AU, which is exactly $149,597,870,700 \mathrm{~km}$, as introduced in 2012 by the International Astronomical Union (IAU) and recognized as a Système International (SI) unit in 2014 by the International Bureau of Weights and Measures (BIPM). Figure 2-3 shows the Earth's orbit in relation to the northern hemisphere's seasons, caused by the average $\approx 23.5^{\circ}$ tilt of the Earth's rotational axis with respect to the plane of the orbit. The solar irradiance available at the top of atmosphere (TOA) is called the extraterrestrial radiation (ETR). ETR (see Equation 2-1) is the power per unit area, or flux density, in Watts per square meter $\left(\mathrm{W} / \mathrm{m}^{2}\right)$, radiated from the sun and available at the TOA. Just like ETS, ETR varies with the Earth-sun distance $(r)$ and annual mean distance $\left(\mathrm{r}_{0}\right)$ :

$$
\mathrm{ETR}=\mathrm{TSI}\left(\mathrm{r}_{0} / \mathrm{r}\right)^{2}
$$

\footnotetext{
${ }^{2}$ See http://www.nrel.gov/midc/spa/.
} 


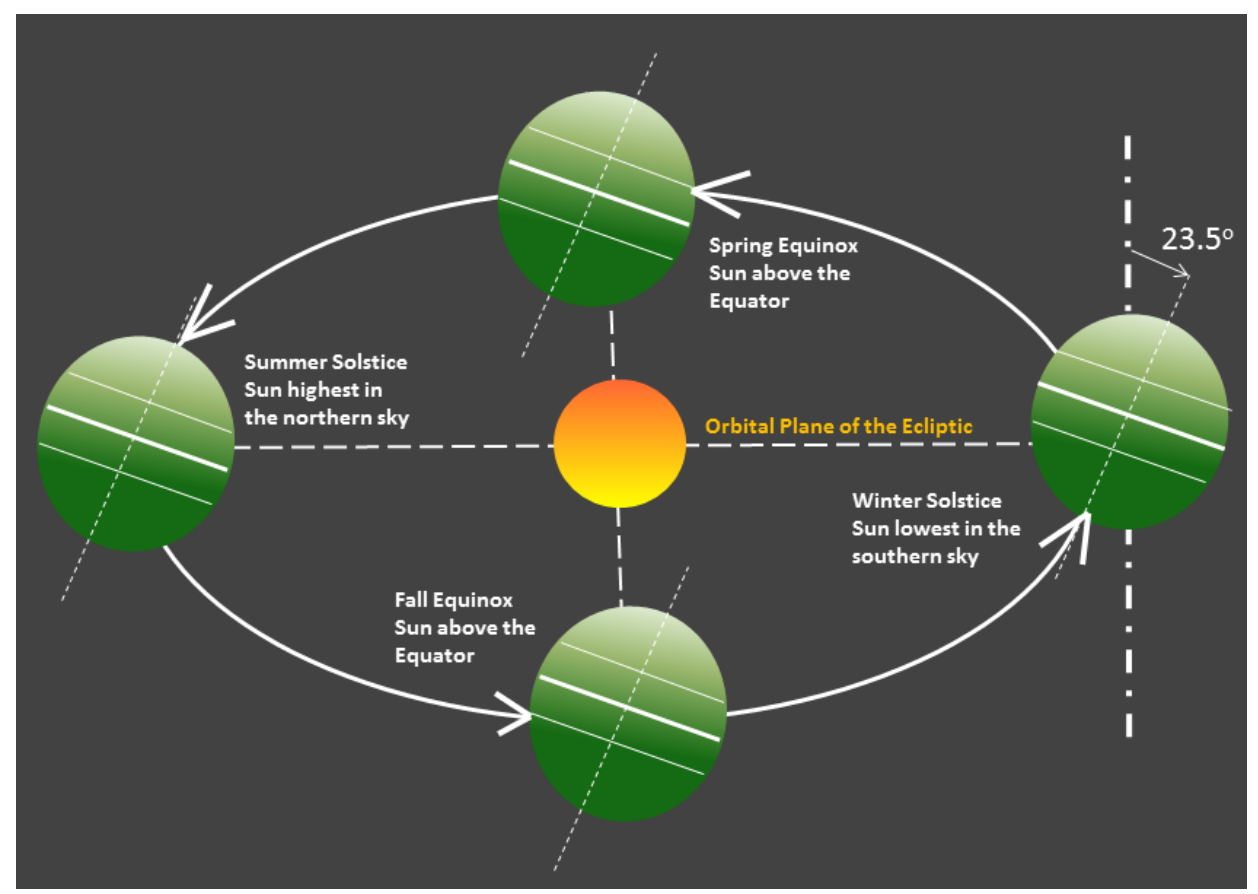

Figure 2-3. Schematic of the Earth's orbit. Image by NREL

From the top of the atmosphere, the sun appears as a very bright disk with an approximate angular diameter of $0.5^{\circ}$ (the actual diameter varies by a small amount as the Earth-sun distance varies) surrounded by a completely black sky (apart from the light coming from stars and planets). This angle can be determined from the distance between the Earth and the sun and the sun's visible diameter. A point at the top of the Earth's atmosphere intercepts a cone of light from the hemisphere of the sun facing the Earth with a total angle of $0.5^{\circ}$ at the apex and a divergence angle from the center of the disk of $0.266^{\circ}$ (half the apex angle, yearly average). Because the divergence angle is very small, the rays of light from the sun are nearly parallel; these are called the solar beam. The interaction of the solar beam with the terrestrial atmosphere is discussed next.

\subsection{Solar Radiation and the Earth's Atmosphere}

The Earth's atmosphere is a continuously variable filter for the solar ETR as it reaches the surface. Figure 2-4 illustrates the "typical" absorption of solar radiation by ozone, oxygen, water vapor, and carbon dioxide. The amount of atmosphere the solar photons must traverse, also called the atmospheric path length or air mass (AM), depends on the relative position of the observer with respect to the sun's position in the sky (Figure 2-4). By convention, air mass one (AM1) is defined as the amount of atmospheric path length observed when the sun is directly overhead. As a first approximation, and for low zenith angles, AM is geometrically related to the solar zenith angle (SZA) because AM = secant of SZA, or $1 / \cos (\mathrm{SZA})$. Because SZA is the complement of the solar elevation angle, $A M$ is also equal to $1 /$ sin (solar elevation angle). Air mass two (AM2) occurs when SZA is $\approx 60^{\circ}$ and has twice the path length of AM1. By extrapolation, one refers to the value at TOA as AM0. 
The cloudless atmosphere also contains gaseous molecules, dust, aerosols, and particulates, for example, which reduce the ETR as it moves through the atmosphere. This reduction is caused mostly by scattering (essentially a complex sort of reflection) and also by absorption (capturing the radiation). Finally, clouds are the major elements that modify the ETR (also by scattering and absorption) on its way to the surface or to a solar collector.

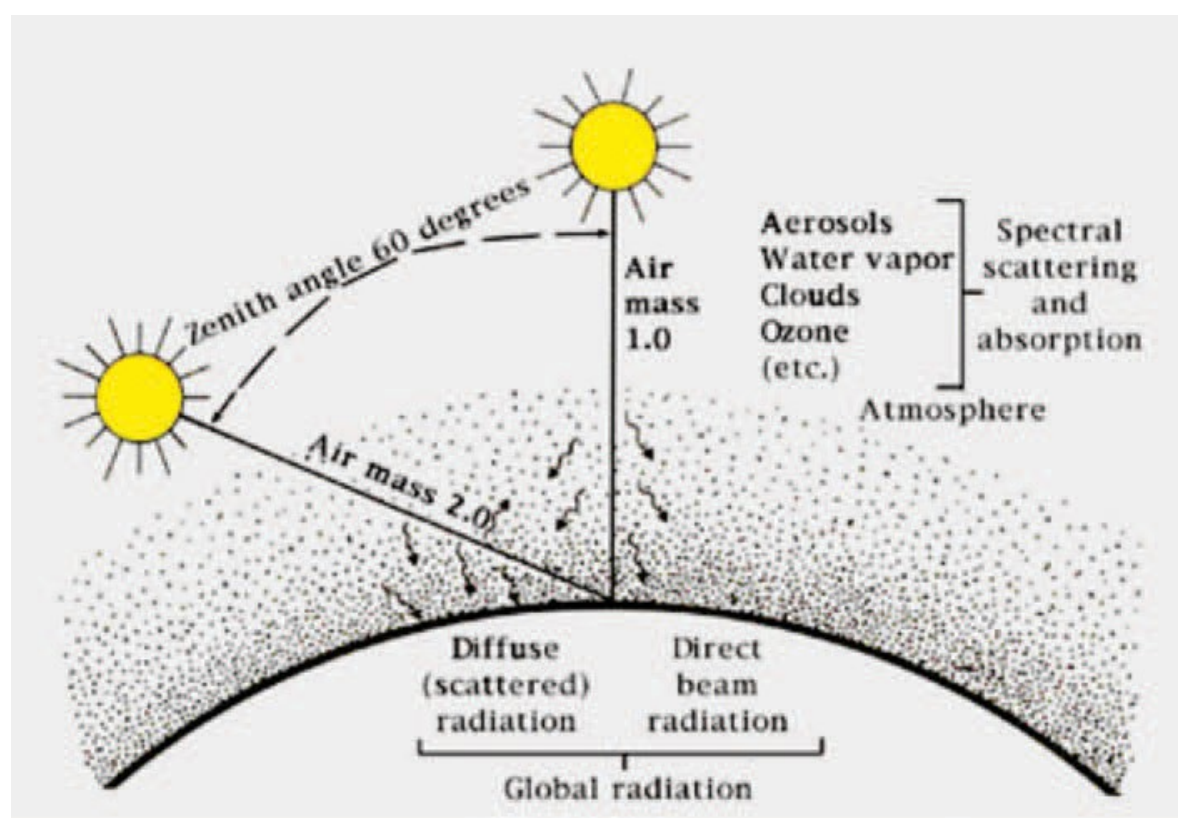

Figure 2-4. Scattering of the direct-beam photons from the sun by the atmosphere produces diffuse radiation that varies with AM (Marion et al. 1992). Image by NREL

Absorption converts part of the incoming solar radiation into heat and raises the temperature of the absorbing medium. Scattering redistributes the radiation in the hemisphere of the sky dome above the observer, including reflecting part of the radiation back into space. The longer the path length through the atmosphere, the more radiation is absorbed and scattered. The probability of scattering - and hence of geometric and spatial redistribution of the solar radiation-increases as the path (AM) from the TOA to the ground increases.

Part of the radiation that reaches the Earth's surface is eventually reflected back into the atmosphere. A fraction of this actually returns to the surface, through a process known as backscattering. The actual geometry and flux density of the reflected and scattered radiation depend on the reflectivity and physical properties of the ground and constituents in the atmosphere, especially clouds and aerosols.

Based on these interactions between the radiation and the atmosphere, the terrestrial solar radiation is divided into two components: direct beam radiation, which refers to solar photons that reach the surface without being scattered or absorbed, and diffuse radiation, which refers to photons that reach the observer after one or more scattering events with atmospheric constituents. These definitions and their usage for solar energy are discussed in detail in Section 2.7 . 
Ongoing research continues to better understand the properties of atmospheric constituents, ways to estimate them, and their impact on the magnitude of solar radiation in the atmosphere at various atmospheric levels and at the surface. This is of great importance to those who measure and model solar radiation fluxes (see Chapters 3-5).

\subsubsection{Relative Motions of the Earth and Sun}

The amount of solar radiation available at the TOA is a function of TSI and the Earth-sun distance at the time of interest. The slightly elliptical orbit of the Earth around the sun was briefly described above and is shown in Figure 2-3. The Earth rotates around an axis through the geographical north and south poles, inclined at an average angle of $\approx 23.5^{\circ}$ to the plane of the Earth's orbit. The resulting yearly variation in the solar input creates seasonal variations in climate and weather at each location. The axial tilt of the Earth's rotation also results in daily variations in the solar geometry throughout the course of one year.

In the northern hemisphere, at latitudes above the Tropic of Cancer $\left(23.437^{\circ} \mathrm{N}\right)$ near midday, the sun is low on the horizon during winter and high in the sky during summer. Summer days are longer than winter days due to progressive changes in where the sun rises and sets. Similar transitions take place in the southern hemisphere. All these changes result in changing geometry of the solar position in the sky with respect to time of year and specific location. The solar position in the sky corresponds to topocentric angles, including:

- The solar elevation is defined as the angle formed by the direction of the sun and the local horizon. Its complementary angle is the SZA.

- The solar azimuth angle is defined as the angle formed by the projection of the direction of the sun on the horizontal plane defined eastward from north, following the International Organization for Standardization (ISO) 19115 standard.

These apparent sun path variations are depicted in Figure 2-5. Because of their significance in performing any analysis of solar radiation data or any radiation model calculation, they must be accounted for by using solar position calculations of sufficient accuracy, such as those derived from NREL's Solar Position Algorithm ${ }^{3}$ (Reda and Andreas 2003, 2004). This algorithm predicts solar zenith and azimuth angles, as well as other related parameters such as the Earthsun distance and the solar declination. All this is possible in the period from $-2,000-6,000$ with an SZA standard deviation of approximately $0.0003^{\circ}(1 ")$. To achieve such accuracy over a long time period, this algorithm is very time consuming, with approximately 2,300 floating operations and more than 300 direct and inverse trigonometric functions at each time step. Other algorithms exist, differing in the attained accuracy and in their period of validity. Various strategies exist to reduce operations, such as reducing the period of validity while still maintaining a high accuracy (Blanc and Wald 2012; Grena 2008; Blanco-Muriel et al. 2001) or keeping a large period while reducing the accuracy (Michalsky 1988).

\footnotetext{
${ }^{3}$ See http://www.nrel.gov/midc/spa/.
} 


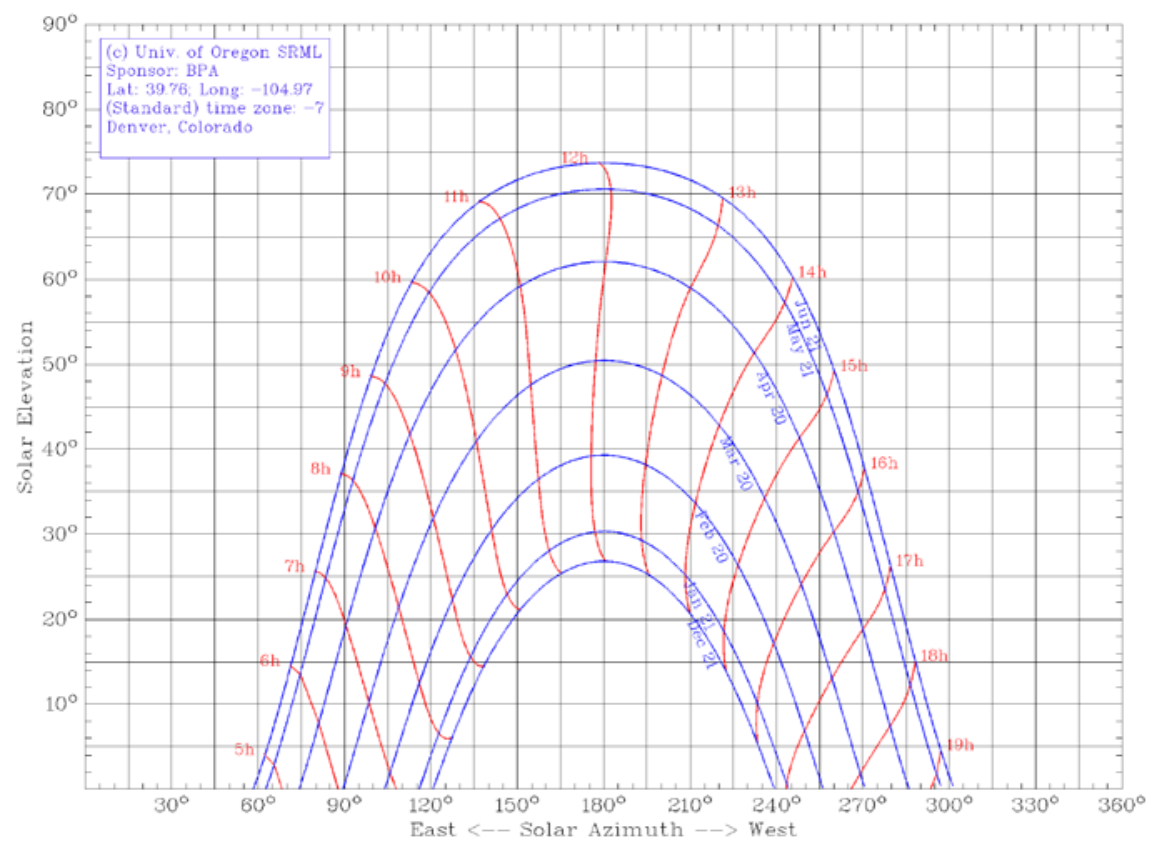

Figure 2-5. Apparent sun path variations during one year in Denver, Colorado. Image from the University of Oregon Solar Radiation Monitoring Laboratory (http://solardata.uoregon.edu/SunChartProgram.php)

\subsection{The Solar Resource and its Components}

Radiation can be transmitted, absorbed, or scattered in varying amounts by an attenuating medium, depending on wavelength (see Figure 2-1). Complex interactions of the Earth's atmosphere with solar radiation result in three fundamental broadband components of interest to solar energy conversion technologies:

- Direct normal irradiance (DNI) - Solar (beam) radiation from the sun's disk itself (of particular interest to concentrating solar power [CSP] and concentrating photovoltaic [CPV] technologies)

- Diffuse horizontal irradiance (DHI) - Scattered solar radiation from the sky dome (excluding the sun and thus DNI)

- Global horizontal irradiance (GHI) - Geometric sum of the direct and diffuse components (also called total hemispheric irradiance).

These basic solar components are related to SZA by the fundamental expression

$$
\mathrm{GHI}=\mathrm{DNI} \times \cos (\mathrm{SZA})+\mathrm{DHI}
$$

These three components are shown in Figure 2-6. 


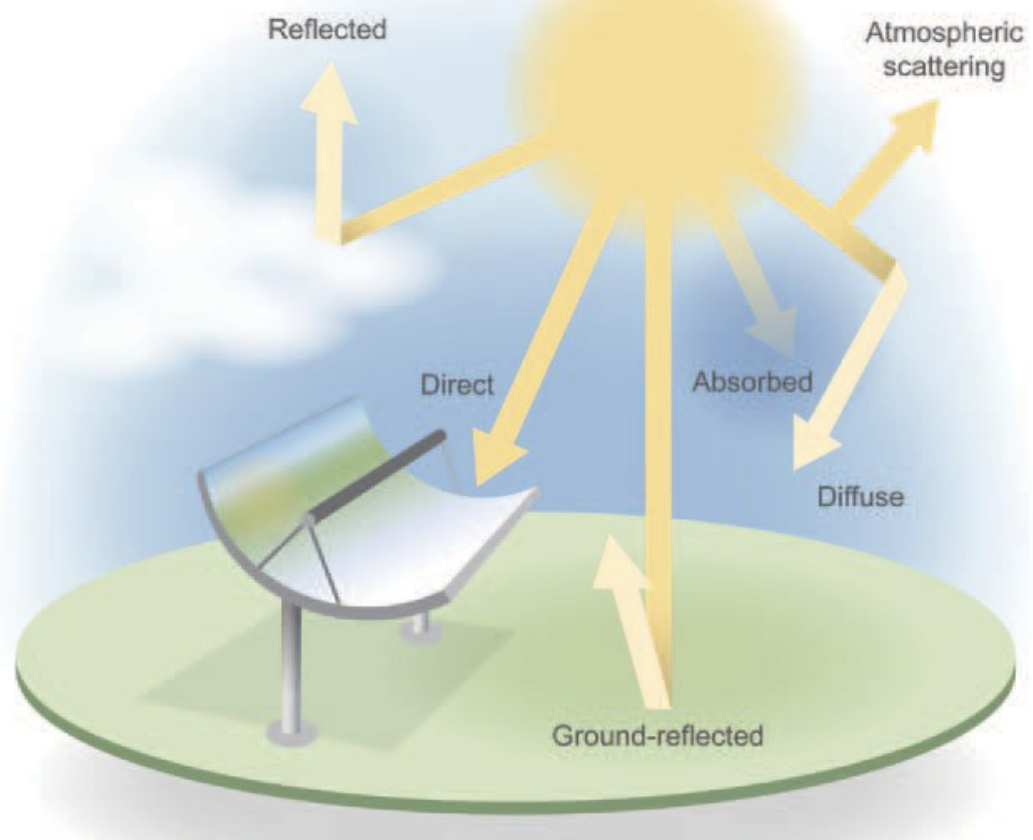

Figure 2-6. Solar radiation components resulting from interactions with the atmosphere. Image by Al Hicks, NREL

\subsubsection{DNI and Circumsolar Irradiance}

By definition, DNI is the irradiance on a surface perpendicular to the vector from the observer to the center of the sun caused by radiation that did not interact with the atmosphere (WMO 2008). This strict definition is useful for atmospheric physics and radiative transfer models, but it results in a complication for ground observations: It is not possible to measure whether or not a photon was scattered if it reaches the observer from the direction in which the solar disk is seen. Therefore, DNI is interpreted in a less stringent way in the world of solar energy. Direct solar radiation is understood as the "radiation received from a small solid angle centered on the sun's disk" (ISO 1990). The size of this "small solid angle" for DNI measurements is recommended to be $5 \cdot 10^{-3} \mathrm{sr}$ (corresponding to $\approx 2.5^{\circ}$ half angle) (WMO 2008). This recommendation is approximately 10 times larger than the radius of the solar disk itself, whose yearly average is $0.266^{\circ}$. This relaxed definition is necessary for practical reasons because instruments for DNI measurements (pyrheliometers) have to track or follow the sun throughout its path of motion in the sky, and small tracking errors are to be expected. The relatively large field of view (FOV) of pyrheliometers reduces the effect of such tracking errors. Similarly, DHI must be obtained by masking the sun with a small shade. An FOV with a radius of $2.5^{\circ}$ is necessary to avoid the impact of tracking errors (e.g., wind-induced tracking errors) and to maintain an FOV complementary to that of the pyrheliometer.

To understand the definition of DNI and how it is measured by pyrheliometers in more detail, the role of circumsolar radiation has to be discussed. Because of forward scattering of direct sunlight in the atmosphere, the circumsolar region closely surrounding the solar disk (solar aureole) looks very bright. The radiation coming from this region is called circumsolar radiation. For the typical 
FOV of modern pyrheliometers $\left(2.5^{\circ}\right)$, circumsolar radiation contributes a variable amount, depending on atmospheric conditions, to the DNI measurement. Determining the magnitude of circumsolar radiation is of particular interest for concentrating solar technologies (CSP or CPV) because it tends to overestimate the yield of most concentrating power plants. This is due to the fact that the FOV of concentrators (typically of the order of $1^{\circ}$ ) is much smaller than that of the pyrheliometers that are used on site to determine the incident DNI.

The circumsolar contribution to the observed DNI can be quantified if the radiance distribution within the circumsolar region and the so-called penumbra function of the pyrheliometer are known. The latter is normally a characteristic of the instrument and can be derived from the manufacturer's data. The former, however, is difficult to determine experimentally with current instrumentation. For instance, a method based on two commercial instruments (a sun and aureole measurement system and a sun photometer) was presented (Gueymard 2010; Wilbert et al. 2013). Other instruments that can measure the circumsolar irradiance are documented in Wilbert et al. (2012, 2013), Kalapatapu et al. (2012), and Wilbert (2014), but all these solutions are expensive and also require additional modeling effort.

To avoid such sophisticated and costly instrumentation, substantial modeling effort would be required with input data that are rarely accessible in real time, particularly in the case when a thin ice cloud (cirrus) reduces DNI but considerably increases the circumsolar contribution. Considering these difficulties, more research would be necessary before the circumsolar fraction can be easily determined by analysts at any location and any instant in their practice of solar resource assessments. For more details, the reader is referred to the detailed discussion (based on both experimental and modeling results) that can be found in Blanc et al. (2014).

\subsubsection{Diffuse Irradiance}

A cloudless atmosphere absorbs and scatters radiation out of the direct beam before it reaches the Earth's surface. Scattering occurs in essentially all directions, away from the specific path of this beam radiation. This scattered radiation is what constitutes the sky diffuse radiation in the hemisphere above the surface. In particular, the Rayleigh scattering theory explains why the sky appears blue (short wavelengths, in the blue and violet parts of the spectrum, are scattered more efficiently by atmospheric molecules) and why the sun's disk appears yellow-red at sunrise and sunset (blue wavelengths are mostly scattered out of the direct beam, whereas the longer red wavelengths are mostly unscattered, resulting in a red shift). The sky radiation in the hemisphere above the local surface is the DHI. A more technical and practical definition of DHI is that it represents all radiation from the sky dome except what is considered DNI. Hence, in practice, DHI is the total diffuse irradiance from the whole sky hemisphere minus the $2.5^{\circ}$ annulus around the sun center.

DHI includes radiation scattered by molecules (Rayleigh effect), aerosols (Mie effect), and clouds (if present). It also includes the backscattered radiation that is first reflected by the surface and then re-reflected downward by the atmosphere or clouds. The impact of clouds is difficult to model because they have optical properties that can vary rapidly over time and can also vary considerably over the sky hemisphere. Whereas a single and homogenous cloud layer can be modeled with good accuracy, complex three-dimensional cloud scenes cannot. 


\subsubsection{Global Irradiance}

The total hemispherical solar radiation on a horizontal surface, or GHI, is the sum of DHI and the projected DNI to the horizontal surface, as expressed by Equation 2-2. This fundamental equation is the basis of most solar radiation measurement system designs, data quality assessments, and atmospheric radiative transfer models addressing the needs for solar resource data. Because GHI is easier to measure than DNI or DHI, most radiometric stations in the world only provide GHI data. It is then necessary to estimate DNI and DHI by using an appropriate conversion model, as discussed in the next section.

\subsubsection{Solar Radiation Resources for Solar Energy Conversion}

Obtaining data time series or temporal averages of the solar radiation components - most importantly GHI and DNI - that relate to a conversion system is the first step before that system can be simulated and its design criteria and performance evaluated. Systems with concentrating optics rely solely on DNI. Low-concentration systems may also be able to use some sky diffuse radiation. Flat-plate collectors, fixed or tracking, can use all radiation components as well as radiation reflected from the ground if in the collector's FOV.

Solar radiation data are required at all stages of a solar project. Before construction, long time series of historical data are necessary to quantify the solar resource and its variability. During operation, real-time data are typically necessary to verify the performance of the system and detect problems. In both cases, the required data may be obtained from measurement, modeling, or a combination of both. Actually, measurements are usually not exclusively used for different reasons: (1) long time series of measured data do not exist at most locations in the world; (2) even if they exist, they most likely contain gaps that must be filled by modeling; and (3) conducting quality measurements is considerably more costly than operating models (assuming, of course, that the otherwise prohibitively high costs of satellite operations and data management are borne by other agencies). Quality measurements remain essential, however, because their uncertainty is normally significantly lower than that of modeled data, and thus, they can serve to validate models and even improve the quality of long-term modeled time series through a "site adaptation" process described in Section 7.3.3

GHI is measured at a relatively large number of stations in the world. However, the quality of such data remains to be verified at the vast majority of these stations. Assuming that good-quality GHI data are available at a station of interest, how can the analyst derive the two other components-DNI and DHI? There are two possible solutions to this frequent situation. The first one is to temporarily ignore the existing GHI data and obtain time series of GHI, DNI, and DHI from a reputable source of satellite-derived data. The modeled and measured GHI data can then be compared for quality assurance and possible bias corrections to the modeled data or, conversely, to determine the quality of the measured data. The second solution consists in using one of the numerous "separation" or "decomposition" models, about which considerable literature exists. Gueymard and Ruiz-Arias (2016) reviewed 140 such models and quantified their performance at 54 high-quality radiometric stations over all continents, using data with high temporal resolution (1minute in most cases). Previous evaluations had targeted a limited number of models, exclusively using the more conventional hourly resolution (e.g., Ineichen 2008; Jacovides et al. 2010; Perez et al. 1990; Ruiz-Arias et al. 2010). All current models of this type being empirical in nature are not of "universal" validity and thus might not be optimized for the specific location under scrutiny, 
particularly under adverse situations (e.g., sub-hourly data or high surface albedo) that can trigger significant biases and random errors. Hence, the most appropriate way to deal with the component separation problem cannot be ascertained. The solar radiation scientific research community, peer-reviewed publications, and published reports are presently used to evaluate, validate, and assess the quality of these conversion algorithms. In general, the higher the time resolution, the larger random errors in the estimated DNI or DHI will be. Even large biases can appear at sub-hourly resolutions if the models used are not appropriate for that. This issue is discussed by Gueymard and Ruiz-Arias $(2014,2016)$, who showed that not all hourly models are appropriate for higher temporal resolutions and that large errors may occur under cloudenhancement situations. A new avenue of research is to optimally combine the estimates from multiple models using advanced artificial intelligence techniques (Aler et al. 2017). More information on this type of calculation is provided in Chapter 4.

\subsubsection{Terrestrial Solar Spectra}

Many solar energy applications rely on collectors or systems that have a pronounced spectral response. This is most importantly the case of the solar cells that constitute the building blocks of photovoltaic (PV) systems. Each solar cell technology has a specific spectral dependence. To allow the comparison and rating of solar cells or modules, it is thus necessary to rely on reference spectral conditions. To this end, various international standardization bodies (ASTM, IEC, and ISO) have promulgated standards that describe such reference terrestrial spectra. In turn, these spectra are mandated to test the performance of any solar cell, using either indoor or outdoor testing methods. Currently, all standard reference spectra are for an air mass of 1.5 (noted AM1.5). The reason for this, as well as historical perspectives on the evolution of these standards, is discussed by Gueymard et al. (2002). The standard reference spectra of relevance to the solar energy community are the following:

- $\quad$ ASTM G173, for DNI and GHI on a $37^{\circ}$-tilted surface

- $\quad$ ASTM G197, for DNI, DHI, and GHI on surfaces tilted at $20^{\circ}$ and $90^{\circ}$

- IEC 60904-3, similar to ASTM G173 with only slightly different values, lower by $0.29 \%$

- ISO 9845-1, an earlier version of ASTM G159, now deprecated and replaced by G173; ISO is currently in the process of preparing an update.

In addition, ASTM G177 defines a "high-UV" spectrum for material degradation purposes.

\subsection{Spatial and Temporal Variability of the Solar Resource}

Variability is a wide-ranging term that may characterize the solar resource in many ways, either from a spatial or temporal perspective. In the latter case, all temporal scales can be considered depending on context, from sub-second to multi-year scales. Temporal variability, if well characterized for a climate region, can be useful to determine the suitability of a short-term data set to produce valid long-term statistics. For instance, the term can be applied to refer to the inter-annual variability of the resource. The example in Figure 2-7 shows the inter-annual variability in monthly DNI in Daggett, California. The coefficient of variation (COV; the ratio of the standard deviation to the mean of a set of given averages) can be used to quantify this variability. Studies of GHI and DNI distributions in the United States show that the range of GHI's inter-annual variability is typically $8 \%-10 \%$. This is generally approximately half or less of the variability of 
DNI, which can be $15 \%$ or more at the $68 \%$ confidence interval (Gueymard and Wilcox 2011). Long-term trends in GHI and DNI are also of importance because of the succession of periods known as "dimming" and "brightening," which affect both climate change and the extrapolation of the historical solar resource into the future (Müller et al. 2014; Wild et al. 2015).

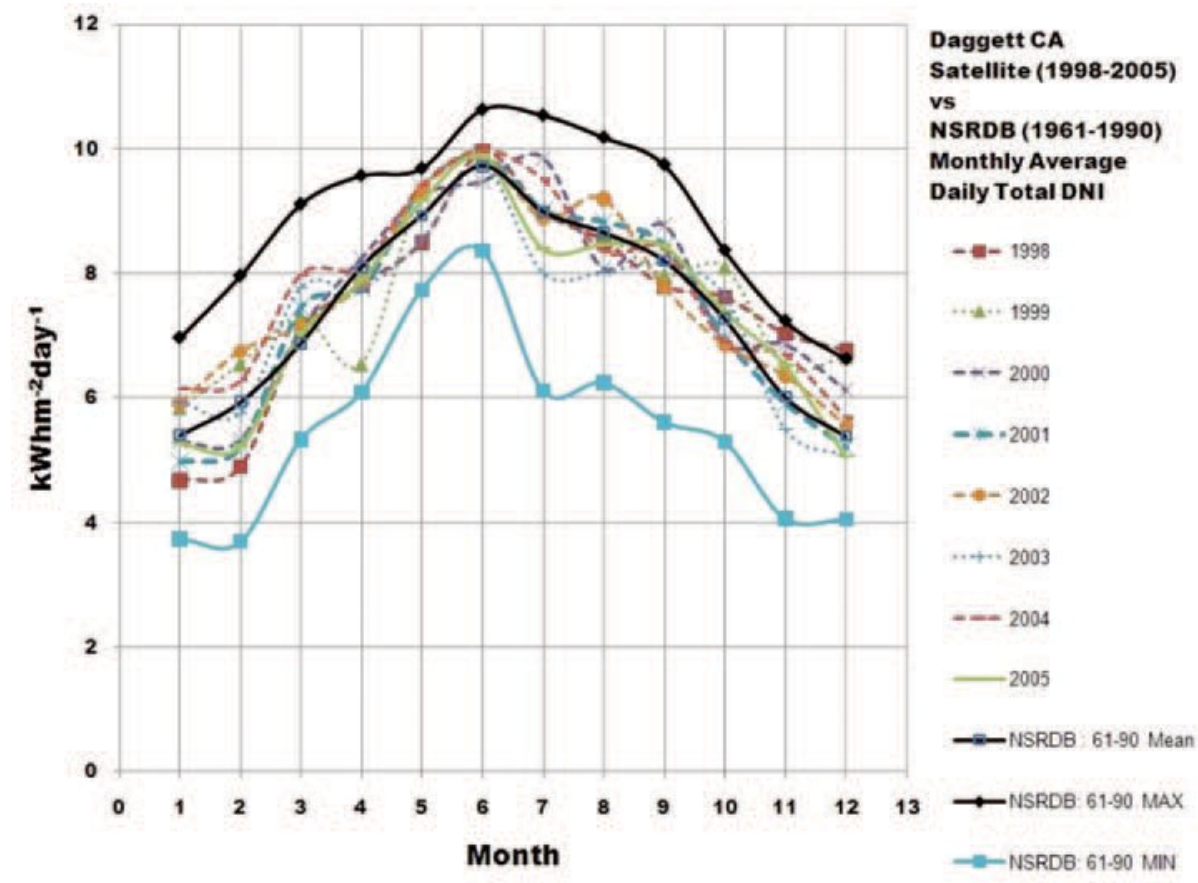

Figure 2-7. Example of direct-beam monthly average daily total $\left(\mathrm{kWh} / \mathrm{m}^{2} /\right.$ day) inter-annual variability from 1961-2005 in Daggett, California. Data from Wilcox et al. (2007). Image by NREL

The term "variability" is also used to describe the spatial variability of the resource in a climatological context. Spatial variability can help determine the applicability of a particular data set for a nearby location, possibly saving the need for additional measurements. In this case, variability characterizes microclimatic features and regional resource gradients. An example is provided in Figure 2-8, which shows the climatological GHI resource distribution over the Island of Oahu in Hawaii. Similarly, Figure 2-9 shows the spatial variability of DNI and global tilted irradiance (GTI) over areas of 50x50 km throughout the United States in terms of COV (Gueymard and Wilcox 2011). ${ }^{4}$

\footnotetext{
${ }^{4}$ Such spatial and temporal variability maps are available from http://rredc.nrel.gov/solar/new_data/variability/.
} 

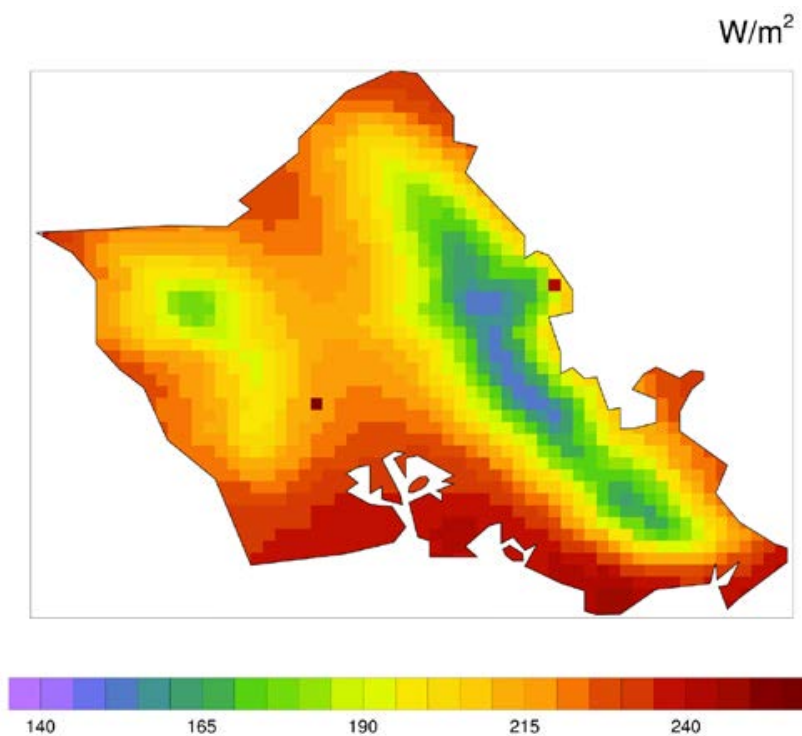

Figure 2-8. Example of microclimatic spatial variability for the Island of Oahu. The 1-km highresolution map displays mean hourly GHI. Image from SolarAnywhere V3.0, 2015
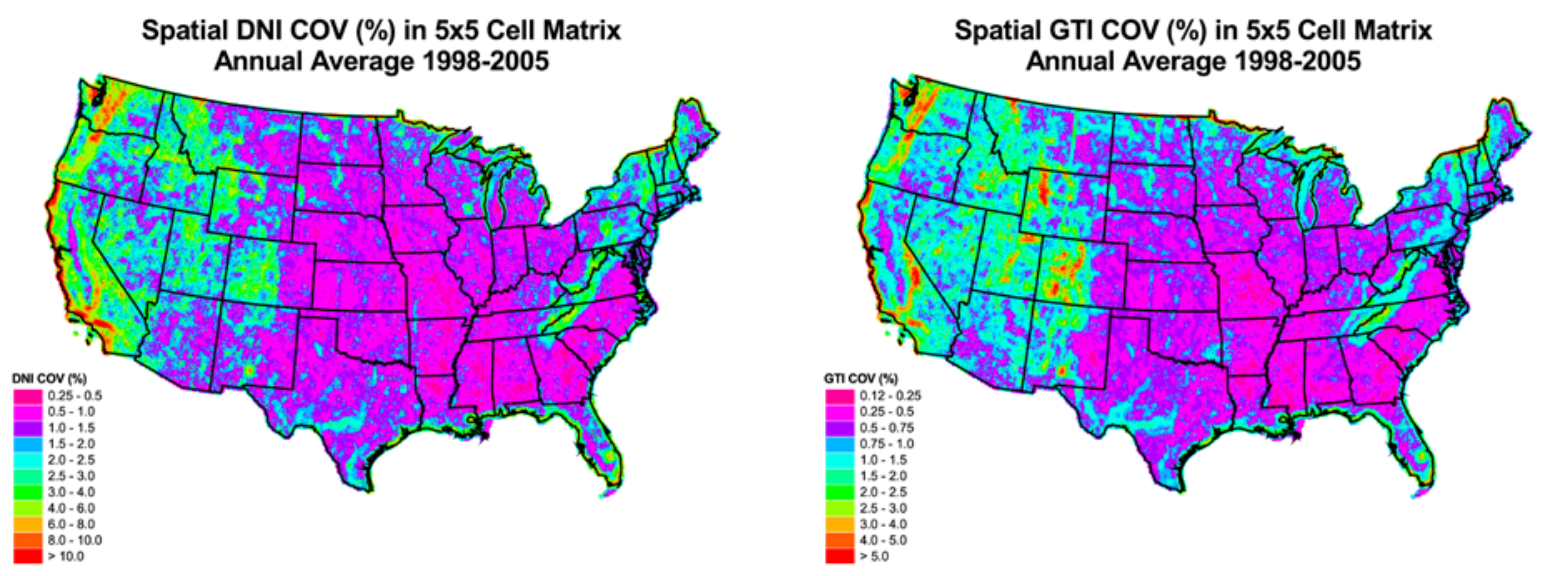

Fig. 2-9. Spatial variability in DNI (left) and GTI (right) over the continental United States, in terms of COV. Images from Gueymard and Wilcox, 2011, Image by NREL

More fundamentally, however, the term variability also refers to the time/space impact of weather and the cycle of days and seasons on the output of solar systems. This output can vary from zero to full power, outside the control of plant operators. Understanding the solar resource's variability is key to optimally integrate the power output of PV systems onto electrical grids. This is discussed further in Section 2.8.2.

Space/time variability has two causes. One is fully predictable and is the result of the apparent seasonal and daily motion of the sun in the sky and the Earth's distance from the sun. The other is less predictable. It is the result of the motion of clouds and, to a lesser extent, of aerosols, in relation to weather systems. 
It is useful to first have a look at the temporal and spatial scales involved and how they impact the resource.

Temporal scale: Beginning with an intuitive example (Figure 2-10), a single location on a partly cloudy day will experience a high degree of temporal variability due to changes in the sun's position and the motion of clouds. However, the solar energy accumulated over several days at that same location exhibits less variability. Variability in GHI becomes small as the temporal integration increases to one year and beyond, but that in DNI or GTI can still be significant (Gueymard and Wilcox 2011; see also Vignola 2001).

Spatial scale: Increasing the solar generation footprint from a single location to a region, and even to a continent, reduces intermittency considerably. Increasing this footprint to the entire planet eliminates it almost entirely (Figure 2-11). This spatial integration effect is often referred to as the "smoothing effect," which is discussed below.

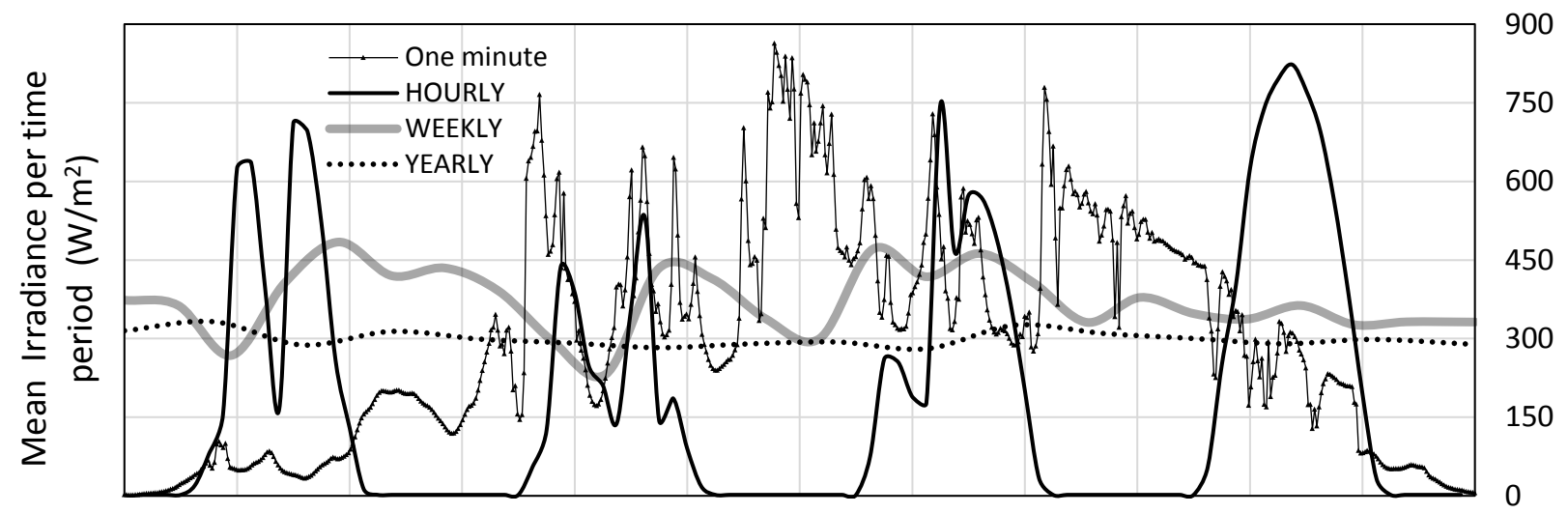

Fig. 2-10. Variability of global irradiance time series at a North American location, as a function of integration time. The figure includes one day of 1-minute data, 4 days of hourly data, 26 weeks of weekly data, and 16 years of yearly integrated data. Image from Perez et al. (2016)

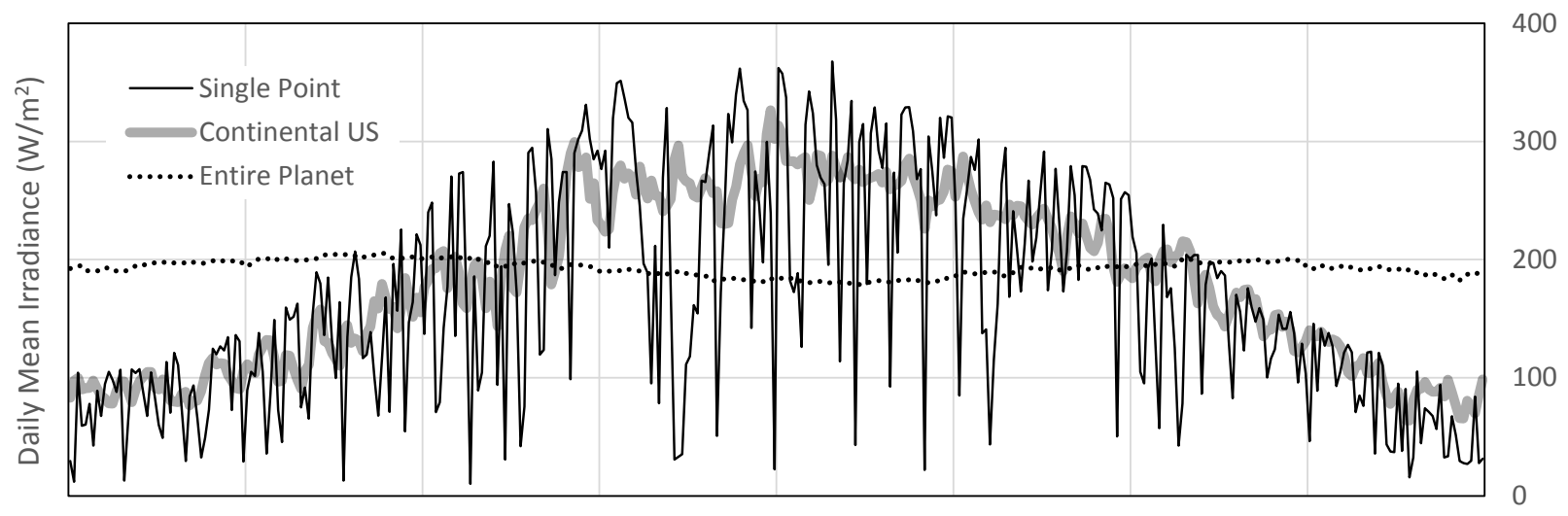

Fig. 2-11. Variability of daily global irradiance time series for one year as a function of the considered footprint. Image from Perez et al. (2016) 


\subsubsection{Quantifying Variability}

The variability quantifying metric should adapt to a wide range of temporal and spatial scales and embed: (1) the physical quantity that varies, (2) the variability time scale, and (3) the time span over which variability is assessed.

Physical quantity: For energy producers and grid operators, the pertinent quantity is the power output, $\boldsymbol{p}$, of a power plant or of a fleet of power plants at a given point in time. The power output variability reflects the underlying variability of irradiance impinging on the plant(s). Therefore, understanding and quantifying the variability of irradiance amounts to quantifying and understanding the variability of $\boldsymbol{p}$. DNI's variability is relevant for concentrating technologies, whereas the variability in GHI or GTI is representative of flat-plate technologies. This section focuses on the latter.

Solar geometry-induced variability is fully predictable. Here, the focus is on cloud/weatherinduced variability that is stochastic in nature. In order to better understand this variability component, it is useful to first remove the solar-geometry effects. The clearness index, Kt (ratio between GHI and its extraterrestrial counterpart), or the clear-sky index, Kt* (ratio between GHI and its clear-sky counterpart), both embed the stochastic variability of irradiance but are largely independent of solar geometry. The use of $K t^{*}$ is preferable in general because it more effectively removes solar geometry effects at low solar elevations (Perez et al. 1990). Nevertheless, its use implies that the clear-sky irradiance can be accurately estimated, which represents an additional step that many analysts try to avoid.

Time scale: The intuitive temporal example presented above suggests that the temporal scale of the selected physical quantity's time series, $\Delta t$, is a fundamental factor. Depending on the application, $\Delta t$ can range from one second or less to hours and more. The change of $K t^{*}$ corresponding to the selected time scale $\Delta t$ is noted as $\Delta K t_{\Delta t}^{*}$. On short scales (milliseconds to minutes), this change is often referred to as the ramp rate.

Time span: A proper measure of variability should include ramp events covering a statistically significant time span. This time span should be a large multiple of $\Delta t$.

Nominal variability metric: Nominal variability refers to the variability of the selected dimensionless clear-sky index. The maximum or mean $\Delta K t_{\Delta t}^{*}$ ramp rate over a given time span has been proposed as such a measure (Hoff and Perez 2010). However, most authors have recently settled on the ramp rate's variance, or its square root- the ramp rate standard deviation —over a given time span as the preferred metric for variability.

$$
\text { Nominal Variability }=\sigma\left(\Delta K t_{\Delta t}^{*}\right)=\sqrt{\operatorname{Var}\left[\Delta K t_{\Delta t}^{*}\right]}
$$

Power output (absolute) variability metric: Eqation 2-3 describes a nominal dimensionless metric. When dealing with power generation, it is necessary to scale up the nominal metric and quantify power variability in absolute terms. This is expressed by Equation 2-4.

$$
\text { Power Variability }=\sigma\left(\Delta \boldsymbol{p}_{\Delta t}\right)=\sqrt{\operatorname{Var}\left[\Delta \boldsymbol{p}_{\Delta t}\right]}
$$


Recall that $\boldsymbol{p}$ can be modeled from $K t^{*}$ via extraction of GHI, extrapolation of plane-of-array irradiance, and inclusion of PV specifications (i.e., without changing the inherent cause of variability). Hence, Equation 2-4 does not include additional intrinsic variability information relative to Equation 2-3.

\subsubsection{Variability Impacts}

Both the temporal variability and the spatial variability are specific to a site (or area) and period. Temporal variability may change seasonally (e.g., from low variability during summer to higher variability [due to increased cloudiness] during winter). The two types of variability directly affect solar resource analyses for various reasons, including:

- Measured data sets often contain data gaps due to instrument failure or various sources of error. To avoid any discontinuity in the time series, analysts are typically tempted to use some form of temporal interpolation to fill the gaps. This is convenient but significantly increases the overall uncertainty.

- If no onsite measurements exist at the project's site, but some do exist at one or more "nearby" sites some distance away, analysts are tempted to extrapolate or average the data from those alternate sites. Depending on distance and spatial variability over that region, this may or may not introduce significant errors.

- Inter-annual and long-term variability (decadal trends) need to be considered to correctly project the measurements or modeled data of the past into the future for design and bankability purposes.

This explains why an evaluation of variability is an important step for accurate solar resource assessments.

From an application perspective, the solar resource variability translates into power production variability, which may impact the stability of electric grids, for instance. One important question that has received specific attention is: How much is the temporal variability at one PV site correlated with that of another PV site some distance away? A high correlation would tend to destabilize the grid, which needs to be asserted.

Based on extensive studies (Hoff and Perez 2012, 2013), it appears that the variability of a fleet of $N$ PV plants over a given region will be reduced by the inverse of the square root of $N$ if the plants' variability is uncorrelated and if they experience similar natural variability. This is a consequence of the spatial smoothing effect noted by many (e.g., Marcos et al. 2012; Murata et al. 2009; Woyte et al. 2007; Wiemken et al. 2001). This result means that nearby locations are highly correlated, experiencing the same ramp rates at nearly the same time and varying in sync. In contrast, the time series from distant locations are uncorrelated. Partial correlation exists between these two extremes. Hoff and Perez (2012b) used 10-km hourly satellite-derived irradiances over the continental United States. They observed a similar asymptotic decay with distance and a predictable dependence of this decay upon $\Delta t$ for time intervals of 1,2 , and 3 hours. They also noted that the rate of decrease of correlation with distance was different for various U.S. regions and attributed these differences to prevailing regional cloud speeds. This is confirmed by Hoff and Norris (2010). Perez et al. (2011) analyzed high-resolution, highfrequency satellite-derived irradiances $(1 \mathrm{~km}, 1$ minute $)$ in climatically distinct regions of North 
America and Hawaii to investigate the site-pair correlation decay as a function of distance, time scale, and mean monthly regional cloud speed (see Figure 2-12), itself independently derived from satellite cloud-motion vectors. Interestingly, as shown in Figure 2-12 for various areas and periods, the rate of decrease of this correlation with distance is a strong function of the data's temporal resolution. A distance of $\approx 5 \mathrm{~km}$ may be sufficient to smooth out fluctuations on a 1minute time scale, whereas distances over $50 \mathrm{~km}$ would be needed to smooth out hourly fluctuations. 


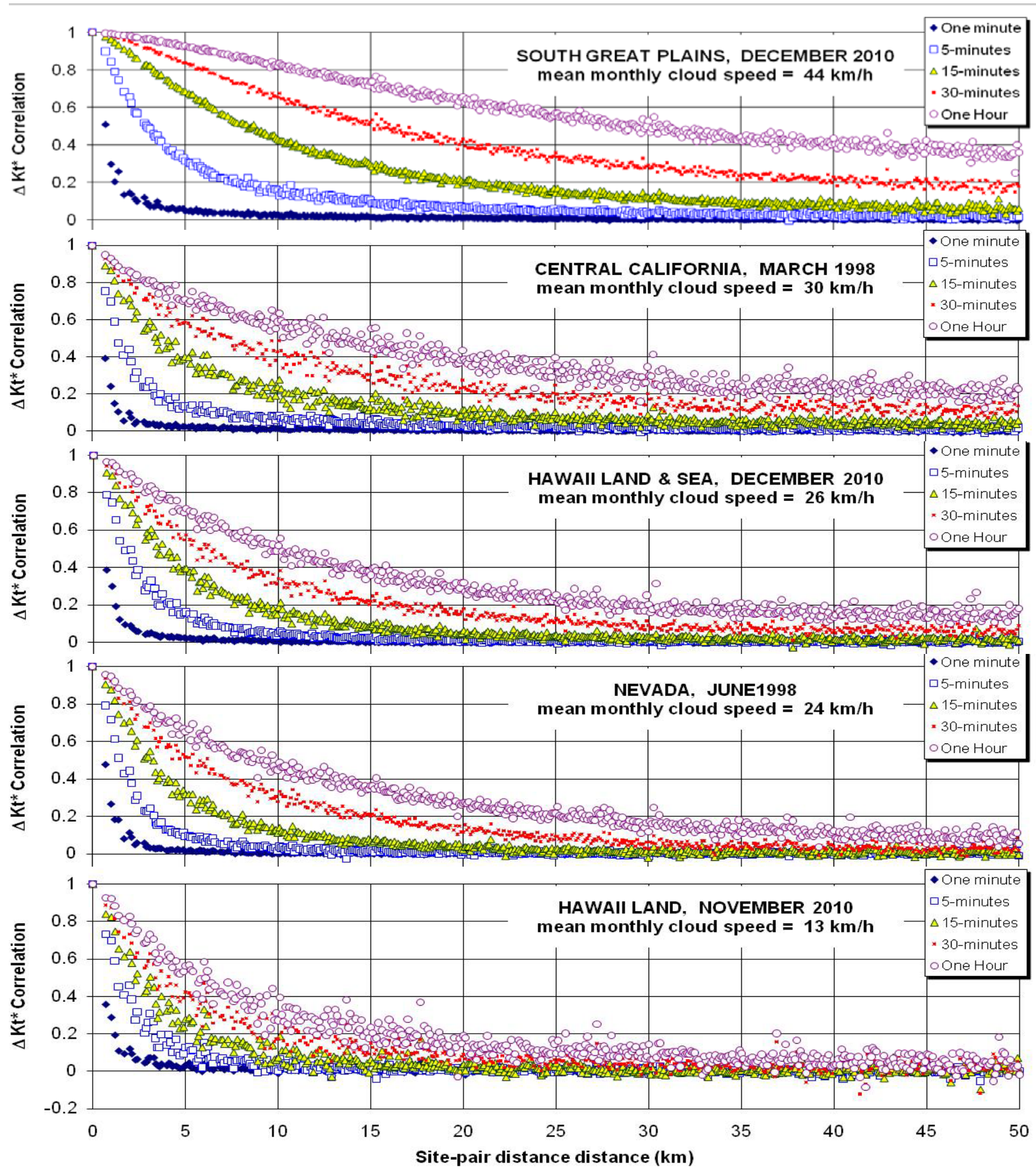

Figure 2-12. Site-pair correlation as a function of time period and distance for sample regions in North America and Hawaii. Mean monthly cloud speed was estimated from satellite-derived cloud motion vectors computed for each data point. Image from Perez et al. (2011) 


\section{References}

Aler, R., I. M. Galván, J. A. Ruiz-Arias, and C. A. Gueymard. 2017. "Improving the Separation of Direct and Diffuse Solar Radiation Components Using Machine Learning by Gradient Boosting." Solar Energy 150: 558-69.

Blanc, P., and L. Wald. 2012. "The SG2 Algorithm for a Fast and Accurate Computation of the Position of the Sun for Multi-Decadal Time Period." Solar Energy 88(10): 3072-83. doi; 10.1016/j.solener.2012.07.018

Blanc, P., Espinar, B., Geuder, N., Gueymard, C., Meyer, R., Pitz-Paal, R., Reinhardt, B., Renné, D., Sengupta, M., Wald, L., Wilbert, S., 2014. "Direct normal irradiance related definitions and applications: The circumsolar issue.” Solar Energy, 110, 561-577, ISSN 0038-092X, http://dx.doi.org/10.1016/j.solener.2014.10.001.

Blanco-Muriel, M., D. C. Alarcón-Padilla, T. López-Moratalla, and M. Lara-Coira. 2001. "Computing the Solar Vector." Solar Energy 70(5): 431-441.

Gueymard, C.A. 2004. “The Sun's Total and Spectral Irradiance for Solar Energy Applications and Solar Radiation Models.” Solar Energy 76: 423-452.

_. 2006. "Reference Solar Spectra: Their Evolution, Standardization Issues, and Comparison to Recent Measurements." Advances in Space Research 37: 323-40. doi:10.1016/j.asr.2005.03.104.

- 2010. "Spectral Circumsolar Radiation Contribution to CPV." Proceedings CPV6 Conference, Freiburg, Germany.

- 2012. "Solar Radiation, Introduction." In: Encyclopedia of Sustainability Science and Technology, R.A. Meyers (Ed.), Springer.

Gueymard, C. A., D. Myers, and K. Emery. 2002. "Proposed Reference Irradiance Spectra for Solar Energy Systems Testing.” Solar Energy 73(6): 443-67.

Gueymard, C.A., and J. A. Ruiz-Arias. 2014. "Performance of Separation Models to Predict Direct Irradiance at High Frequency: Validation Over Arid Areas." In: Proc. EuroSun 2014, ISES, Aix-les-Bains, France.

_. 2016. "Extensive Worldwide Validation and Climate Sensitivity Analysis of Direct Irradiance Predictions From 1-Min Global Irradiance.” Solar Energy 128: 1-30.

Gueymard, C., and S. Wilcox. 2011. "Assessment of Spatial and Temporal Variability in the US Solar Resource From Radiometric Measurements and Predictions From Models Using GroundBased or Satellite Data." Solar Energy 85(5): 1068-84.

Hoff, T.E., and B. Norris. 2010. "Mobile High-Density Irradiance Sensor Network: Cordelia Junction Results. Personal Communication." 
Hoff, T. E., and R. Perez. 2010. “Quantifying PV power Output Variability.” Solar Energy 84: 1782-93.

Hoff, T., and R. Perez. 2012. “Modeling PV Fleet Output Variability.” Solar Energy 86(8): 2177-89.

Kalapatapu, R., M. Chiesa, P. Armstrong, and S. Wilbert. 2012. "Measurement of DNI Angular Distribution With a Sunshape Profiling Irradiometer." Proc. SolarPACES Conf., Marrakech, Morocco.

Marcos, J., L. Morroyo, E. Lorenzo, and M. Garcia. 2012. "Smoothing of PV Power Fluctuations by Geographical Dispersion." Prog. Photovolt: Res. Appl. 20: 226-37.

Michalsky, J. 1988. "The Astronomical Almanac's Algorithm for Approximate Solar Position (1950-2050)." Solar Energy 40(3): 227-35.

Müller, B., M. Wild, A. Driesse, and K. Behrens. 2014. "Rethinking Solar Resource Assessments in the Context of Global Dimming and Brightening." Solar Energy 99: 272-82.

Murata, A., H. Yamaguchi, and K. Otani. 2009. "A Method of Estimating the Output Fluctuation of Many Photovoltaic Power Generation Systems Dispersed in a Wide Area." Electrical Engineering in Japan 166(4): 9-19.

Perez, R., P. Ineichen, R. Seals, and A. Zelenka. 1990. "Making Full Use of the Clearness Index for Parameterizing Hourly Insolation Conditions." Solar Energy 45: 111-4.

Perez, R., T. Hoff, and S. Kivalov. 2011. "Spatial \& Temporal Characteristics of Solar Radiation Variability." Proc. of International Solar Energy (ISES) World Congress, Kassel, Germany.

Perez, R., M. David, T. Hoff, S. Kivalov, J. Kleissl, M. Jamaly, P. Lauret, and M. Perez. 2016. "Spatial and Temporal Variability of Solar Energy." Foundations and Trends in Renewable Energy 1(1): 1-44.

Reda, I., and A. Andreas. 2003. Solar Position Algorithm For Solar Radiation Applications. Solar Energy, 76:577-589.

SolarAnywhere. 2015. https://www.solaranywhere.com/. V3.

Vignola, F. 2001. "Variability of Solar Radiation Over Short Time Intervals." Proc. Solar 2001, American Solar Energy Society Conf., Washington, D.C.

Wiemken, E., H. G. Beyer, W. Heydenreich, and K. Kiefer. 2001. "Power Characteristics of PV Ensembles: Experience from the Combined Power Productivity of 100 Grid-Connected Systems Distributed Over Germany." Solar Energy 70: 513-9.

Wilbert, S., R. Pitz-Paal and J. Jaus, Joachim . 2012. "Circumsolar Radiation and Beam Irradiance Measurements for Focusing Collectors.” ES1002: Cost Wire Workshop, 22.-23. May 2012, Risö. 
Wilbert, S., B. Reinhardt, J. DeVore, M. Röger, R. Pitz-Paal, and C. A. Gueymard. 2013. "Measurement of Solar Radiance Profiles With the Sun and Aureole Measurement System." Journal of Solar Energy Engineering 135: 041002.

Wild, M., D. Folini, F. Henschel, N. Fischer, and B. Müller. 2015. "Projections of Long-Term Changes in Solar Radiation Based on CMIP5 Climate Models and Their Influence on Energy Yields of Photovoltaic Systems.” Solar Energy 116: 12-24.

Woyte, A., R. Belmans, and J. Nijs. 2007. "Fluctuations in Instantaneous Clearness Index: Analysis and Statistics.” Solar Energy 81(2): 195-206. 


\title{
3 Measuring Solar Radiation and Relevant Atmospheric Parameters
}

\author{
Stefan Wilbert, ${ }^{1}$ Thomas Stoffel, ${ }^{2}$ Daryl Myers, ${ }^{3}$ Stephen Wilcox, ${ }^{2}$ Aron Habte, ${ }^{4}$ Frank \\ Vignola, ${ }^{5}$ John Wood, ${ }^{6}$ and Luis Martin Pomares ${ }^{7}$ \\ 1. German Aerospace Center (DLR) \\ 2. Solar Resource Solutions, $L L C$ \\ 3. Retired, National Renewable Energy Laboratory \\ 4. National Renewable Energy Laboratory \\ 5. University of Oregon \\ 6. Peak Design Ltd. \\ 7. Qatar Environment and Energy Research Institute (QEERI)
}

Accurate measurements of the incoming irradiance are essential to solar power plant project design and implementation. Because irradiance measurements are relatively complex, and therefore expensive, compared to other meteorological measurements, they are available for only a limited number of locations. This holds true especially for direct normal irradiance (DNI). Developers utilize irradiance data for:

- Site resource analysis

- System design

- Plant operation.

Irradiance measurements are also essential for:

- Developing and testing models that use remote satellite sensing techniques or available surface meteorological observations

- Developing solar resource forecasting techniques.

This chapter focuses on the instrument selection, characterization, installation, design, and operations and maintenance (O\&M), including calibration of measurement systems suitable for collecting irradiance resource measurements for renewable energy technology applications.

\subsection{Instrumentation Selection Options}

Before considering instrumentation options and the associated costs, the user must first evaluate the data accuracy or uncertainty levels that will satisfy the ultimate analyses based on the radiometric measurements. This ensures that the best value can be achieved after considering the various available measurement and instrumentation options

By first establishing the project needs for solar resource data accuracy, the user can base instrument selection and the associated levels of effort necessary to operate and maintain the measurement system on an overall cost-performance determination. Specifically, the most accurate instrumentation (see Section 3.2.2 for descriptions of International Organization for Standardization (ISO) 9060 first class; World Meteorological Organization (WMO) "high quality"; or ISO 9060 secondary standard) should not be purchased if the project resources cannot support the maintenance required to ensure measurement quality consistent with the radiometer design specifications and manufacturer recommendations. In such cases, alternative instrumentation designed for lower maintenance requirements and reduced measurement 
performance, such as radiometers with photodiode-based detectors and diffuser disks or integrated measurement systems such as rotating shadowband irradiometers (RSIs) could produce more consistent results.

Redundant instrumentation is another important consideration to ensure confidence in data quality. Multiple radiometers at the project site and/or providing for the measurement of the solar irradiance components (global horizontal irradiance [GHI], diffuse horizontal irradiance [DHI], DNI, and plane of array [POA]), regardless of the primary measurement need, can greatly enhance opportunities for post-measurement data quality assessment required to provide confidence in the resource data.

Measuring other meteorological parameters relevant to the amounts and types of solar irradiance available at a specific time and location can also provide opportunities for post-measurement data quality assessment (see Section 3.3).

\subsection{Instrument Types}

Instruments designed to measure any form of radiation are called radiometers. The earliest developments of instrumentation for measuring solar radiation were designed to meet the needs of agriculture for bright sunshine duration to understand evaporation and by physicists for determining the solar constant. During the $19^{\text {th }}$ and $20^{\text {th }}$ centuries, the most widely deployed instrument for measuring solar radiation was the Campbell-Stokes sunshine recorder (Iqbal 1983; Vignola, Michalsky, and Stoffel 2012). This analog device focuses the direct beam by a simple spherical lens (glass ball) to create burn marks during clear periods (when DNI exceeds $120 \mathrm{Wm}^{-2}$ ) on a sensitized paper strip placed daily in the focus curve. By comparing the total burn length to the corresponding day length, records of percent possible sunshine from stations around the world became the basis for characterizing the global distribution of solar radiation (Löf et al. 1966). The earliest pyrheliometers (from the Greek for fire, sun, and measure) were based on calorimetry and used by scientists to measure brief periods of DNI from various locations, generally at high elevations to minimize the effects of a thick atmosphere on the transmission of solar radiation. By the early $20^{\text {th }}$ century, scientists had developed pyranometers (from the Greek for fire, above, and measure) to measure GHI for understanding the Earth's energy budget (Vignola, Michalsky, and Stoffel 2012).

This section summarizes the types of commercially available radiometers most commonly used to measure solar radiation resources for solar energy technology applications. Solar resource assessments are exclusively based on broadband measurements (i.e., encompassing the whole shortwave spectrum [0.29-4 $\mu \mathrm{m}]$ ). More specialized instruments (spectroradiometers) are needed to evaluate the spectral distribution of this irradiance, which in turn is useful to investigate the spectral response of photovoltaic (PV) cells, for instance. Such instruments, however, are typically only deployed at research centers and are thus excluded from this presentation.

\subsubsection{Pyrheliometers and Pyranometers}

Pyrheliometers and pyranometers are two types of radiometers used to measure solar irradiance. Their ability to receive solar radiation from two distinct portions of the sky distinguishes their designs. As described in Chapter 2, pyrheliometers are used to measure DNI, and pyranometers are used to measure GHI, DHI, or global tilted irradiance (GTI; also known as POA). Table 3-1 summarizes some key attributes of these two radiometers. 
Table 3-1. Overview of Solar Radiometer Types and Their Applications

\begin{tabular}{|l|l|l|l|}
$\begin{array}{c}\text { Radiometer } \\
\text { Type }\end{array}$ & Measurement & $\begin{array}{c}\text { Field of View } \\
\text { (Full Angle) }\end{array}$ & \multicolumn{1}{|c|}{ Installation } \\
\hline Pyrheliometer & DNI & $5^{\circ}-6^{\circ}$ & $\begin{array}{l}\text { Mounted on automatic solar tracker } \\
\text { for alignment with the solar disk }\end{array}$ \\
\hline Pyranometer & GHI & $2 \pi$ steradians & $\begin{array}{l}\text { Mounted on stable horizontal } \\
\text { surface free from local obstructions }\end{array}$ \\
\hline Pyranometer & DHI & $2 \pi$ steradians & $\begin{array}{l}\text { Mounted on automatic solar tracker } \\
\text { fitted with shading mechanism or on } \\
\text { a manually adjusted shadowband } \\
\text { platform to block DNI from detector } \\
\text { surface }\end{array}$ \\
\hline Pyranometer & GTI & $2 \pi$ steradians & $\begin{array}{l}\text { Mounted in the POA of the flat-plate } \\
\text { solar collector (fixed or tracked in } \\
\text { one or two axes) }\end{array}$ \\
\hline
\end{tabular}

${ }^{a}$ Optionally thermopile pyranometers are installed with powered ventilator and heating system to reduce contamination of optical surfaces and thermal errors. It must be made sure that the base of thermopile pyranometer is shielded from direct sunlight.

Pyrheliometers and pyranometers commonly use either a thermoelectric or photoelectric passive sensor to convert solar irradiance $\left(\mathrm{W} / \mathrm{m}^{2}\right)$ into a proportional electrical signal (microvolts $[\mu \mathrm{V}]$ direct current $[\mathrm{DC}]$ ). Thermoelectric sensors have an optically black coating that allows for a broad and uniform spectral response to all solar radiation wavelengths between approximately $300 \mathrm{~nm}$ and 3,000 nm (Figure 3-1, left). The most common thermoelectric sensor used in radiometers is the thermopile. There are "all-black thermopile sensors" used in pyrheliometers and pyranometers, as well as "black-and-white thermopile pyranometers." In "all-black thermopile sensors" the surface exposed to solar radiation is completely covered by the absorbing black coating. The absorbed radiation creates a temperature difference between the black side of the thermopile and the other side. The temperature difference causes a voltage signal. In black-and-white thermopiles, the surface exposed to radiation is partly black and partly white. In this case the temperature difference between the black and the white surfaces creates the voltage signal. Because of the relatively large thermal mass of thermopiles, their $95 \%$ response times are typically $1-30$ seconds. ${ }^{1}$ That is, the output signal lags the changes in solar flux. Some instruments include a signal post processing that tries to reduce this time lag. Recently, new smaller thermopile sensors with response times of approximately 0.5 seconds have been made commercially available.

In contrast to thermopiles, common photoelectric sensors generally respond to only the visible and near-infrared spectral regions from approximately 400-1,100 nm (Figure 3-1, right; Figure 3-2). Examples of photoelectric sensors are silicon ( $\mathrm{Si}$ ) pyranometers, photodiode pyranometers, and solid-state pyranometers. These sensors have very fast time-response characteristics — on the order of microseconds.

\footnotetext{
${ }^{1}$ The given response time represents the time it takes the instrument to reach $95 \%$ of the final value. Typically, a step-like change of the incoming irradiance is used to determine the response time.
} 
For either thermopile or Si sensor in commercially available instruments, the electrical signal generated by exposure to solar irradiance levels of approximately $1,000 \mathrm{~W} / \mathrm{m}^{2}$ is on the order of 10 millivolts $(\mathrm{mV})$ DC (assuming no amplification of the output signal and an appropriate shunt resistor for photodiode sensors). This rather low-level signal requires proper electrical grounding and shielding considerations during installation. Several manufacturers now offer pyranometers with built-in amplifiers and/or digital outputs.
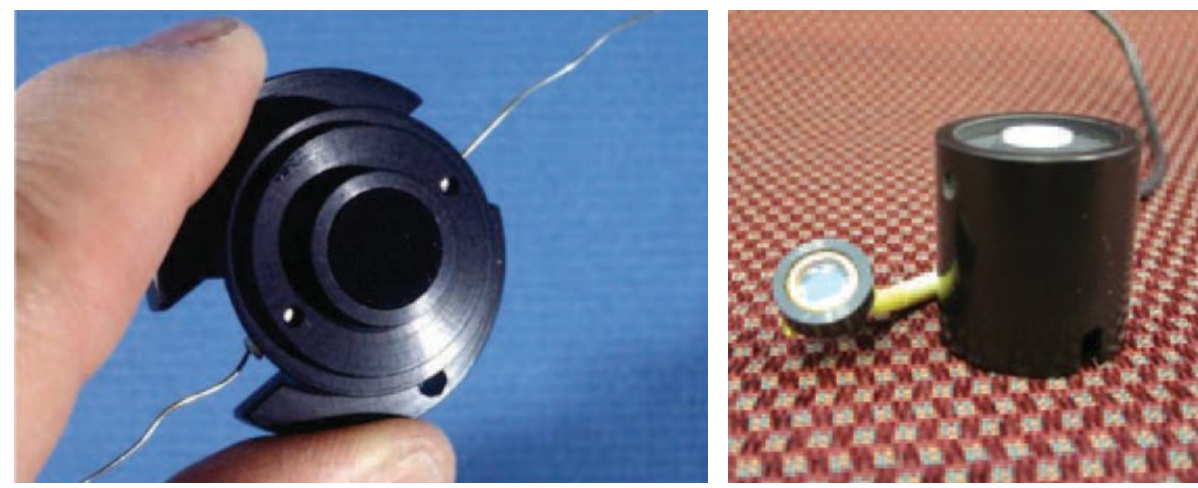

Figure 3-1. (Left) Thermopile assembly used in an Eppley Laboratory, Inc., model precision spectral pyranometer (PSP); (right) typical photodiode detector. Photograph used with permission from LICOR, Inc.

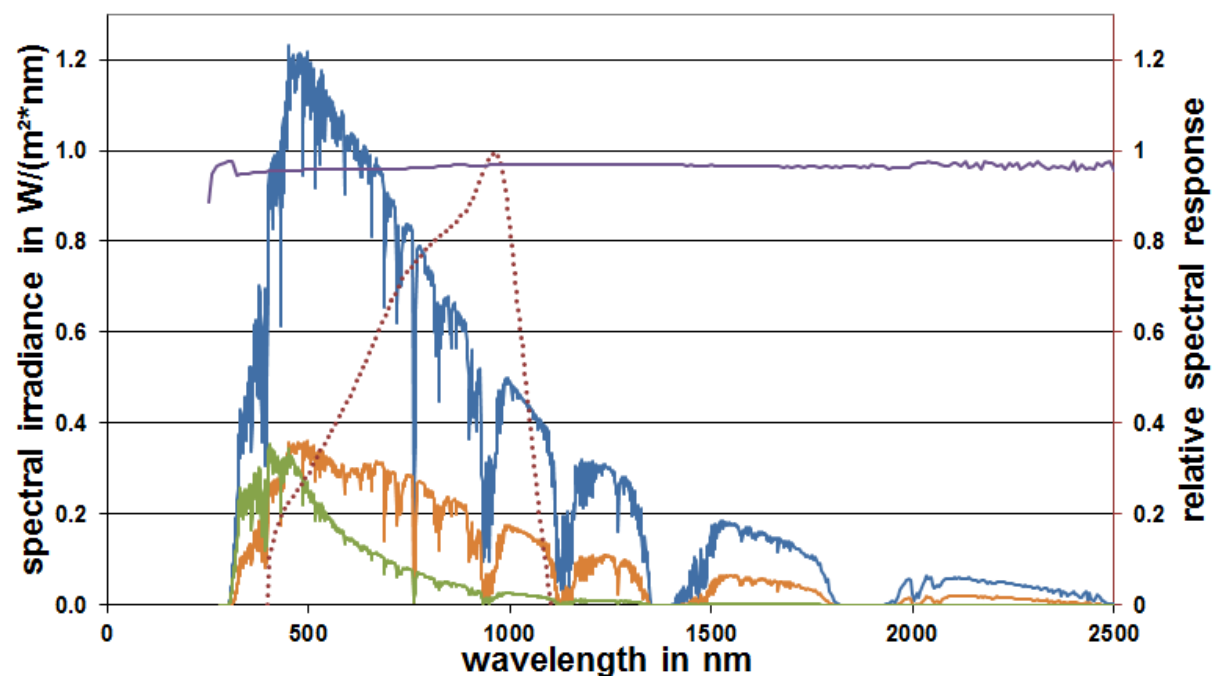

- $\mathrm{GHI}, \mathrm{AM}=1.5$
- $\mathrm{GHI}, \mathrm{AM}=4$
-DHI, AM=1.5
‥ spectral response
LICOR
-spectral response
CM21

Figure 3-2. Spectral response of LI-COR pyranometers LI200SA and Kipp and Zonen CM21 thermopile pyranometer plotted next to different GHI and DHI spectra. Image from Wilbert et al. 2016

\subsubsection{Pyrheliometers}

All modern pyrheliometers should have a $5^{\circ} \mathrm{FOV}$, following the WMO (2014) recommendations. However, the field of view (FOV) of older instruments may be larger, such as $5.7^{\circ}-10^{\circ}$ full angle. Pyrheliometers are mounted on automatic solar trackers to maintain the instrument's alignment with the solar disk and fully illuminate the detector from sunrise to sunset (Figure 3-3 and Figure 3-4). Alignment of the pyrheliometer with the solar disk is determined by a simple diopter - a sighting device in which a small spot of light (the solar image) falls on a mark in the center of a target located near the rear of the instrument (Figure 3-5). Modern sun 
trackers also include software to detect and precisely track the sun position even if the sun is masked. By convention, and to allow for small variations in tracker alignment, view-limiting apertures inside a pyrheliometer allow for the detection of radiation in a narrow annulus of sky around the sun (WMO 2014), called circumsolar region. This circumsolar radiation component is the result of forward scattering of radiation near the solar disk, itself caused by cloud particles, atmospheric aerosols, and other constituents that can scatter solar radiation. Depending on the FOV - or, to be more precise, the sensor's penumbra function (see Section 2.7 on "DNI and Circumsolar Irradiance" and references therein)_and tracker alignment, pyrheliometer measurements include varying amounts of circumsolar irradiance contributions to DNI.

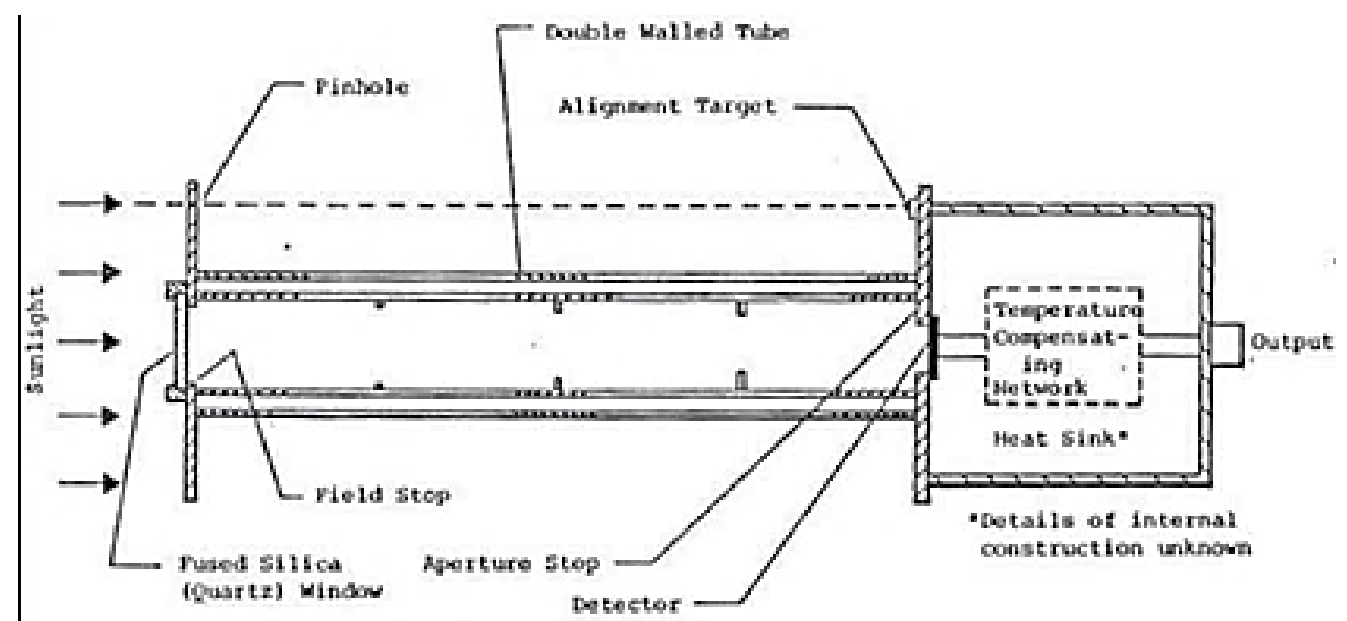

Figure 3-3. Schematic of an Eppley Laboratory, Inc., model normal incidence pyrheliometer (NIP) (Bahm and Nakos 1979). Image from the former U.S. Energy Research and Development Administration, now DOE 


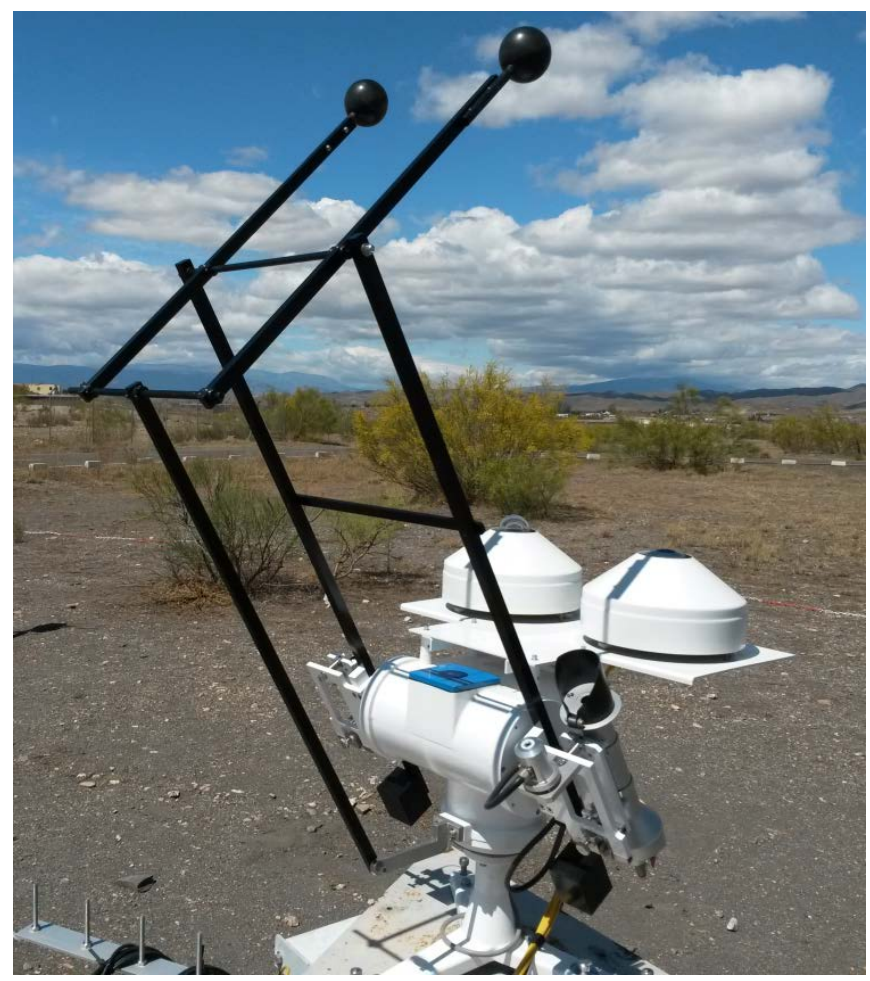

Figure 3-4. Pyrheliometer, a shaded pyranometer and a shaded pyrgeometer mounted on an automatic solar tracker. Photo from DLR
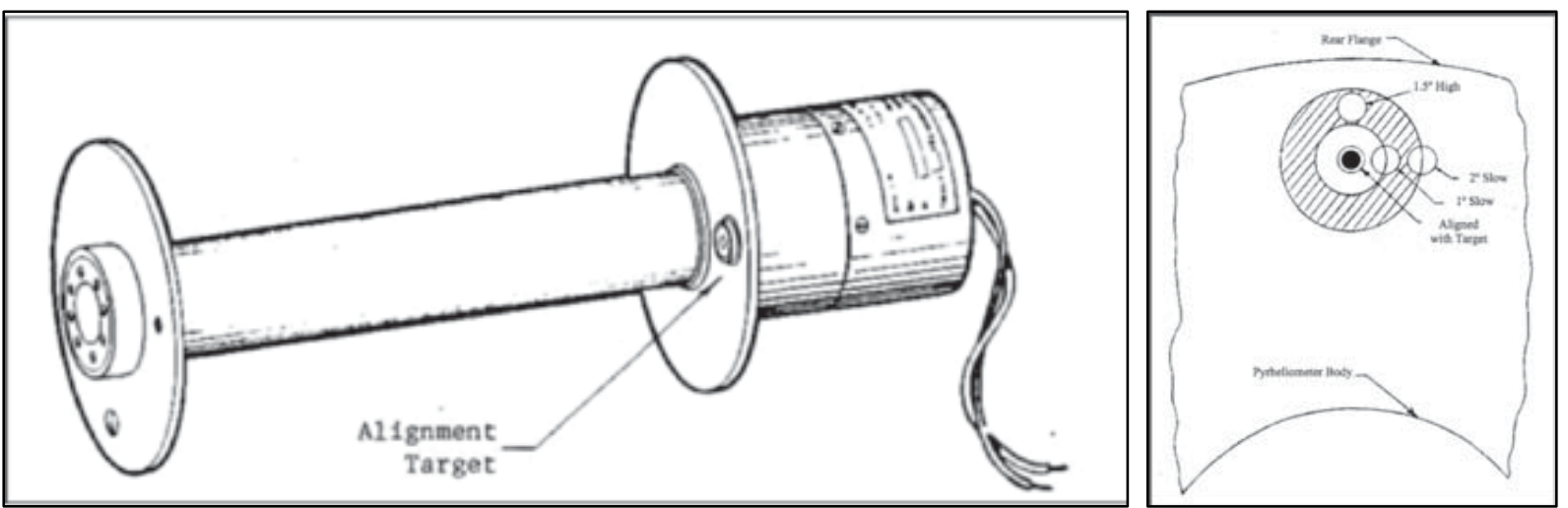

Figure 3-5. Schematics of pyrheliometer alignment diopter configuration including alignment errors for selected positions of the light spot. Image used with permission from Leonard Micek (Micek 1981)

The most accurate measurements of DNI are accomplished using an electrically self-calibrating absolute cavity radiometer (ACR; see Figure 3-6). This advanced type of radiometer is the basis for the World Radiometric Reference (WRR), the internationally recognized detector-based measurement standard for DNI (Fröhlich 1991). The WMO World Standard Group of ACRs is shown in Figure 3-7. By design, ACRs have no windows and are therefore generally limited to fully attended operation during clear-sky conditions to protect the integrity of the receiver cavity (Figure 3-8). Removable windows and temperature-controlled all-weather designs are available for automated continuous operation of these pyrheliometers; however, the installation of the protective window nullifies the "absolute" nature of the DNI measurement. The window 
introduces additional measurement uncertainties associated with the optical transmittance properties of the window (made from either quartz or calcium fluoride) and the changes to the internal heat exchange due to the now sealed system. Moreover, ACRs need some periods of self-calibration during which no exploitable measurement is possible. This creates discontinuities in the high-accuracy DNI time series that could be measured with windowed ACRs, unless a regular pyrheliometer is also present to provide the necessary redundancy (Gueymard and RuizArias 2015). Combined with their very high cost of ownership and operation, this explains why ACRs are not routinely used to measure DNI in the field, with rare exceptions.

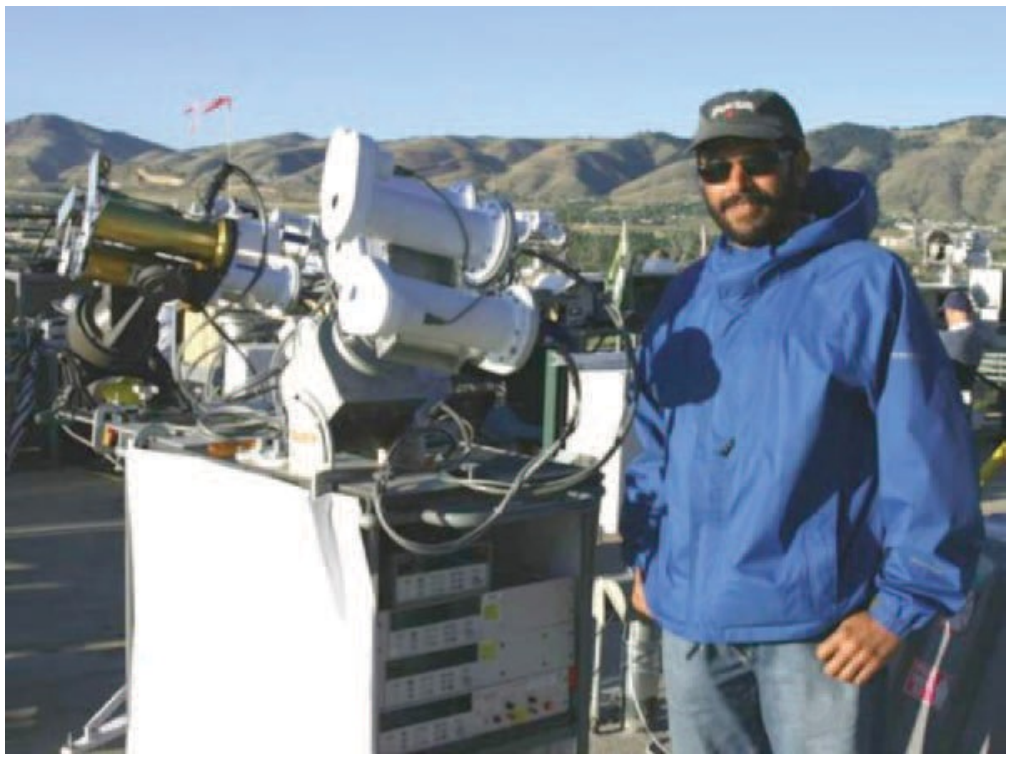

Figure 3-6. Multiple electrically self-calibrating absolute cavity radiometers mounted on solar trackers with control and data acquisition electronics. Image by NREL 


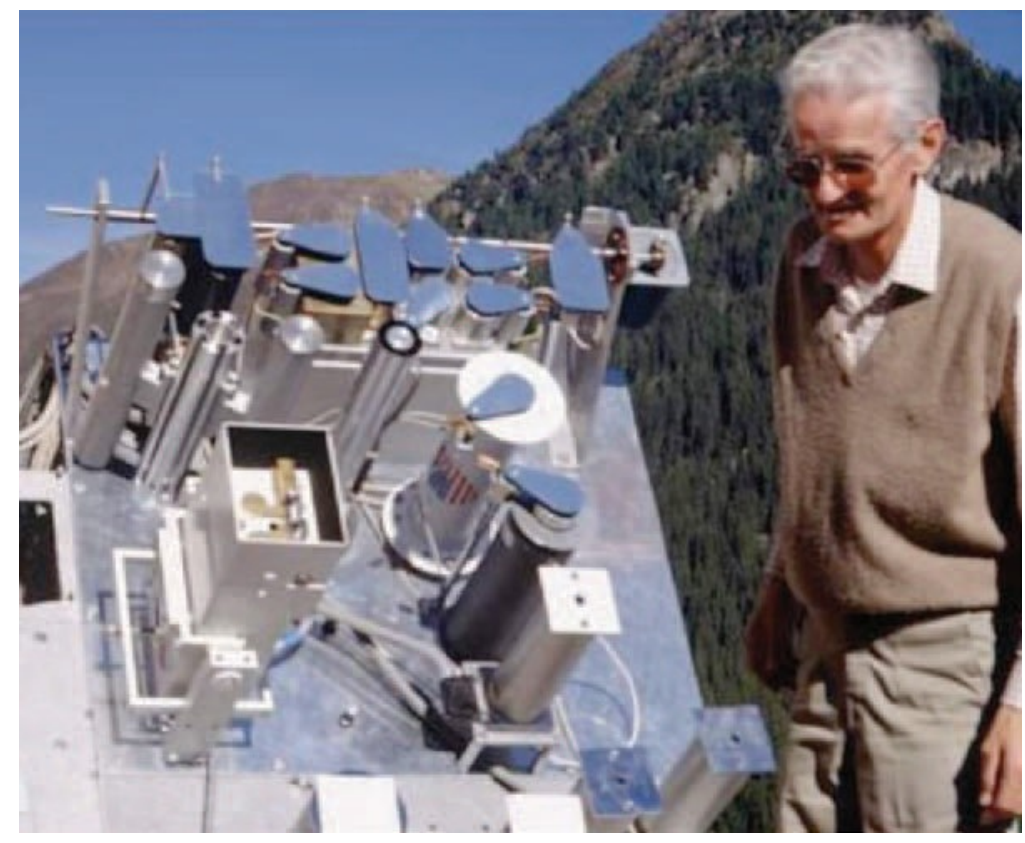

Figure 3-7. The World Standard Group of six absolute cavity radiometers is used to define the WRR or DNI measurement standard. Image by NREL 


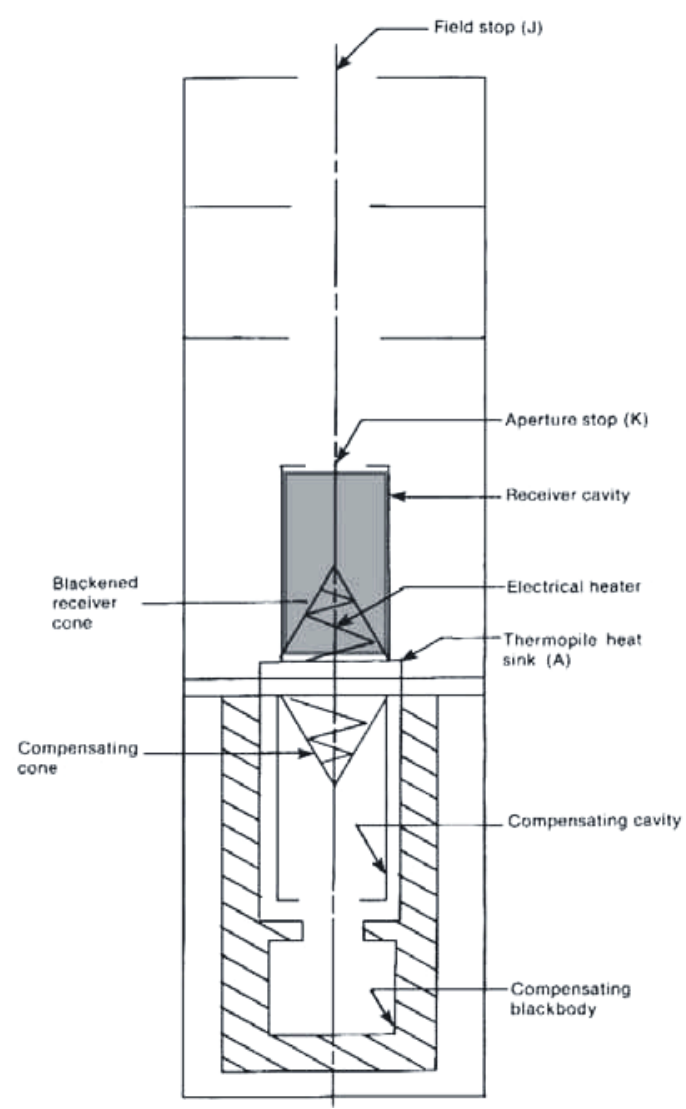

Figure 3-8. Schematic of the Eppley Laboratory, Inc., model automatic Hickey-Frieden (AHF) absolute cavity radiometer. Image modeled from Reda 1996

\subsubsection{Pyranometers}

A pyranometer has a thermoelectric or photoelectric detector with a hemispherical FOV $\left(360^{\circ}\right.$ or $2 \pi$ steradians) (see Figures 3-4 and 3-9). This type of radiometer is mounted horizontally to measure GHI. In this horizontal mount, the pyranometer has a complete view of the sky dome. Ideally, the mounting location for this instrument is free of natural or artificial obstructions on the horizon. Alternatively, the pyranometer can be mounted at a tilt to measure GTI, in the case of, for example, latitude-tilt or vertical solar systems. In an upside-down position, it measures the reflected irradiance. The local albedo is simply obtained by dividing the latter by GHI.

The pyranometer detector is mounted under a protective dome (made of precision quartz or other high-transmittance optical material) or a diffuser. Both designs protect the detector from the weather and provide optical properties consistent with receiving hemispheric solar radiation. Pyranometers can be fitted with ventilators that constantly blow air-sometimes heated-from under the instrument and over the dome (Figure 3-10). The ventilation reduces the potential for contaminating the pyranometer optics caused by dust, dew, frost, snow, ice, insects, or other materials. Ventilators and heating also affect the thermal offset characteristics of pyranometers with single all-black detectors (Vignola et al. 2009). The ventilation devices can, particularly when heated, require a significant amount of electrical power $(5-20 \mathrm{~W})$, adding to the required capacity for onsite power generation in remote areas. Both DC and alternating current (AC) ventilators exist, but current research indicates that DC ventilators are preferable. 
Photodiode detectors employ a diffuser above the detector (Figure 3-11), which is consistent with their low-cost design. Acrylic diffusers can be more dust tolerant than optical glass domes (Maxwell et al. 1999) but may degrade faster over time.

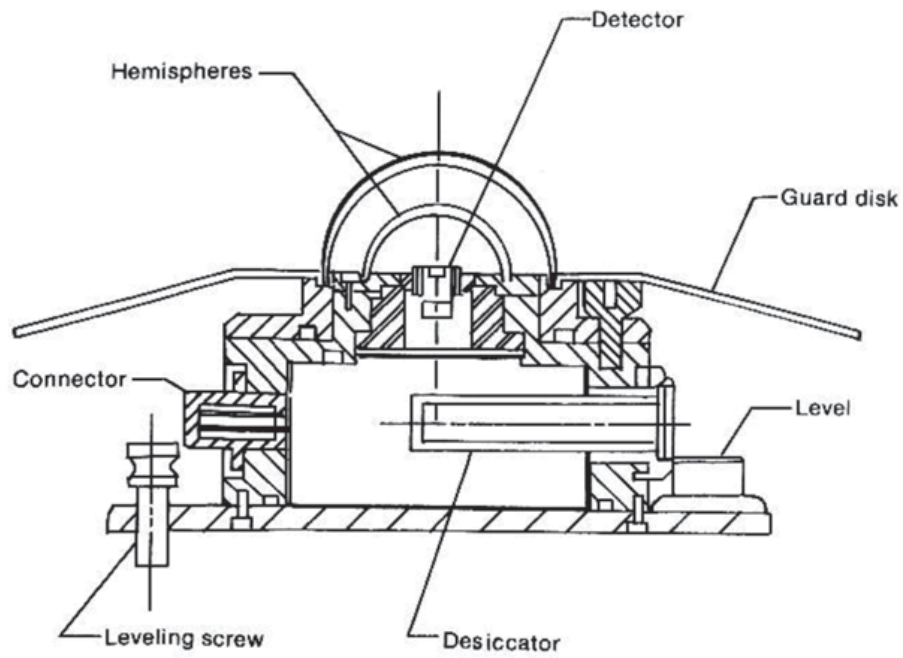

Figure 3-9. Schematic of the Eppley Laboratory, Inc., Precision Spectral Pyranometer (PSP) model. Image by NREL 


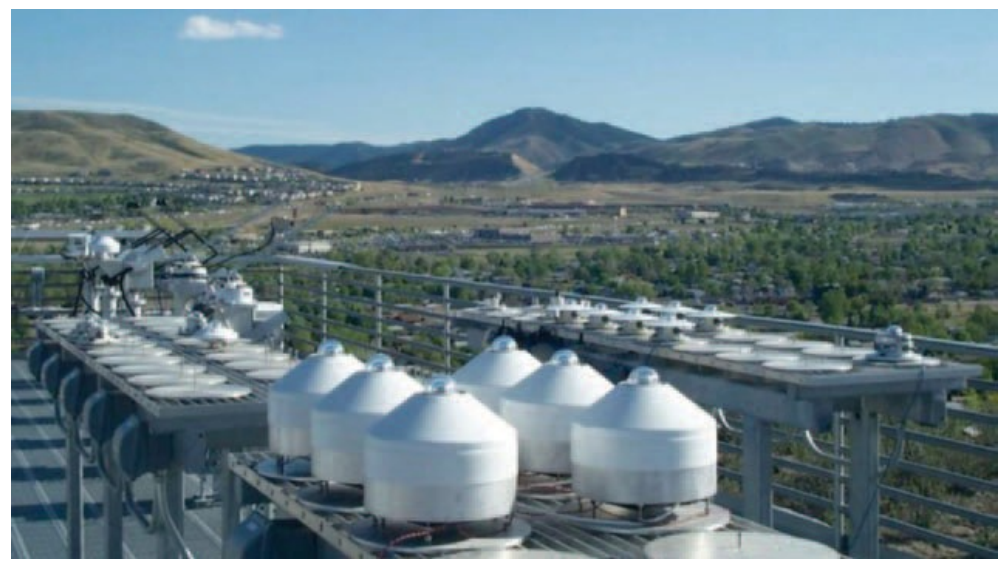

Figure 3-10. Kipp \& Zonen model CM22 pyranometers installed in ventilated CV2 enclosures. Photo by NREL
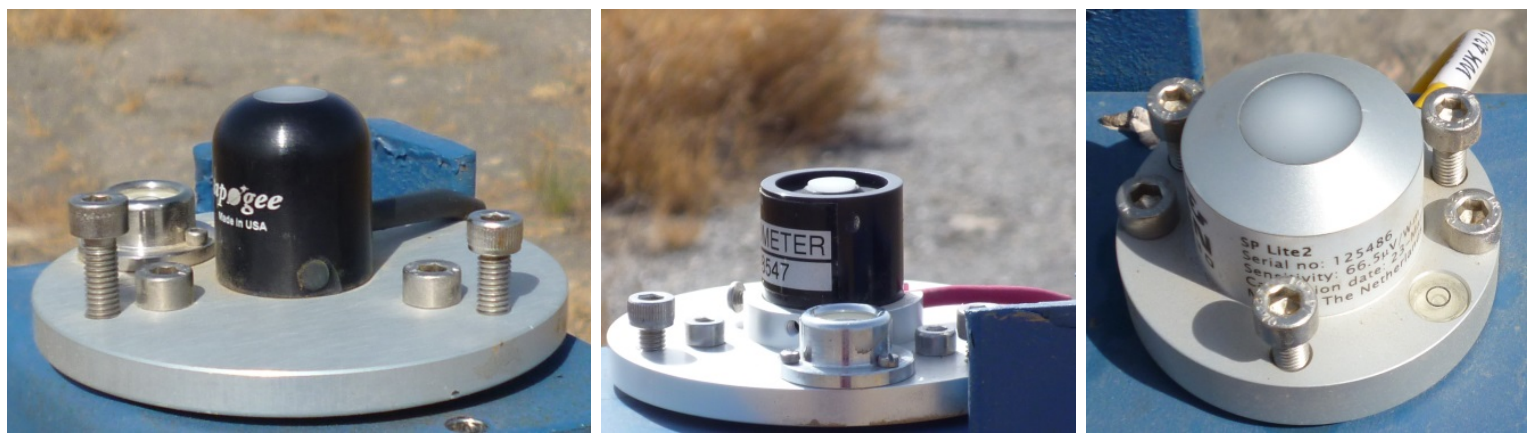

Figure 3-11. Selected photodiode sensors with different diffusor geometries. Photos from Stefan Wilbert, DLR

Pyranometers can also be used to measure the diffuse irradiance. The required device for this measurement is known as a difusometer. It consists of a pyranometer and a shading structure that blocks the direct radiation on its way to the sensor. Shading balls, shading disks, shading rings, or shadowbands are used for that purpose. Shading balls and shading disks must track the sun and cover only a small part of the sky corresponding to the angular region defined for measuring DNI (normally $5^{\circ}$ ). Shading rings cover the complete solar path during a day as seen from the pyranometer. They are actually designed such as to cover the sun's path on consecutive days so that readjustments of the shading ring position are only required every other day. This makes shading rings also block a significant part of sky diffuse radiation. Therefore, correction functions are necessary to determine DHI from the shading ring setup. In turn, this explains why the accuracy of such a DHI determination is lower than that of a DHI measurement with a shading disk or a shading ball. Shadowbands are further described in connection with the RSIs in Section 3.2.5.

\subsubsection{Pyrheliometer and Pyranometer Classifications}

Both ISO and WMO have long established classifications and specifications for the measurement of solar irradiance. The relevant publications are ISO 9060 (ISO 1990) and the Commission for Instruments and Methods of Observation (CIMO) guide (WMO 2014). The reader is encouraged to review these documents in more detail as part of project planning for solar resource measurements before acquiring pyrheliometers or pyranometers. 
Several instrument properties are used as the basis for these pyrheliometer and pyranometer classifications. The WMO characteristics of operational pyrheliometers and pyranometers are presented in Table 3-2 and Table 3-3, respectively. Alternatively, the ISO specifications for these radiometers are presented in Table 3-4 and Table 3-5. These classifications are provided here to address questions about differences in data quality and to give the reader a better understanding of the data quality afforded by particular instrument classes. In the tables, Rs denotes responsivity as, for example, given in $\mu \mathrm{V}$ per $\mathrm{W} / \mathrm{m}^{2}$.

Unfortunately, the current version of ISO 9060 from 1990 and the WMO classification of radiometers have to be considered incomplete and partly outdated. There are several shortcomings in the current classifications:

- Missing categories for fast-response radiometers with solid-state sensors

- Unclear specifications of instrument properties such as the spectral selectivity

- Missing discussion on shading devices for radiometers

- Missing discussion on correction functions for systematic errors

- Contradictions between both classification schemes.

Therefore, working groups have drafted a new American Society for Testing and Materials (ASTM) classification standard and an update of the ISO 9060 standard. Additionally, the recent standard from the International Electrotechnical Commission(IEC), IEC 61724-1 (2017), provides instrumentation guidelines for a particular application (monitoring of PV systems).

Fast sensors with diffusor disks such as pyranometers with small thermopile sensors and $\mathrm{Si}$ photodiode pyranometers are frequently used in solar resource assessments due to their low price, their robustness against sensor soiling, and their fast response time. Whereas some thermopile pyrheliometers and pyranometers recently introduced on the market have sub-second time constants, only fast Si-based sensors can be used for accurate monitoring of extremely rapid fluctuations of solar irradiance. Under such circumstances, typically caused by cloud enhancement events, side-by-side thermopile and Si radiometers may disagree by a significant margin (Gueymard 2017a, 2017b). Si-based sensors were so far excluded from ISO 9060 because of their spectral selectivity. The spectral selectivity was defined in ISO (1990) and McArthur (2005) as the deviation of the product of spectral absorptance and spectral transmittance from the corresponding mean in the range $0.35-1.5 \mu \mathrm{m}$. WMO (2014) uses a significantly broader interval from $0.3-3 \mu \mathrm{m}$ together with strict limits, which are not appropriate for sensor classification. It is important to mention that the spectral selectivity is neither the spectral error nor the spectral range.

Additional classes are proposed in the new draft standards for sensors with response times below 1 second, which include sensors with arbitrary spectral selectivity. The previously existing classes for spectrally nearly flat sensors will be slightly updated. The definition of the spectral selectivity in the new ISO draft is updated to cover solid-state sensors and a more representative wavelength range from $0.33-2.6 \mu \mathrm{m}$. As the final form of these changes is not yet agreed, readers should consult the new standards when they are released. 
As the most accurate way to determine GHI involves the combination of DNI and DHI (ISO 1990; Michalsky et al. 1999), the required shading devices should also be mentioned in a standard. Shading devices such as shading balls, shading disks, shading masks, and rotating shadowbands used in RSIs are also included in the current revised ISO 9060 draft.

As explained above, many radiometers produce systematic errors, and hence, correction functions must be applied to reduce the uncertainties in the measurements. The new draft standards state that, for classification purposes, the final signal of a sensor can be used after application of specific correction functions if a tool to carry out these corrections is provided with the instrument. The corrections can be provided either as a software code for the instrument's internal processors, for the data logger or control units, or as external software. However, only referring to a publication is not acceptable. This prevents the situation that customers would buy an instrument advertised as accurate, to learn later that they can only reach the expected accuracy after investing their own personnel resources to implement the necessary corrections.

Even within each instrument class, there can be some measurement uncertainty variations. The user should research various instrument models to gain familiarity with the design and measurement performance characteristics in view of a particular application (Myers and Wilcox 2009; Wilcox and Myers 2008; Gueymard and Myers 2009; Habte et al. 2014). Furthermore, the accuracy of an irradiance measurement depends on the instrument itself as well as on its alignment, maintenance, data logger calibration, appropriate wiring, and other conditions and effects. The accuracy of radiometers is further discussed in Chapter 6. 
Table 3-2. WMO Characteristics of Operational Pyrheliometers for Measuring DNI ${ }^{\mathrm{a}}$

\begin{tabular}{|c|c|c|c|}
\hline \multicolumn{2}{|c|}{ Characteristic } & $\begin{array}{l}\text { High } \\
\text { Quality }^{\mathrm{a}}\end{array}$ & $\begin{array}{l}\text { Good } \\
\text { Quality }^{a}\end{array}$ \\
\hline \multicolumn{2}{|c|}{ Response time (95\% response) } & $<15 \mathrm{~s}$ & $<30 \mathrm{~s}$ \\
\hline \multicolumn{2}{|c|}{ Zero offset-response to $5-\mathrm{K} / \mathrm{h}$ change in ambient temperature } & $2 \mathrm{~W} / \mathrm{m}^{2}$ & $4 \mathrm{~W} / \mathrm{m}^{2}$ \\
\hline \multicolumn{2}{|c|}{ Resolution—smallest detectable change in $\mathrm{W} / \mathrm{m}^{2}$} & 0.51 & 1 \\
\hline \multicolumn{2}{|c|}{ Stability_change per year, percentage of full scale } & 0.1 & 0.5 \\
\hline \multicolumn{2}{|c|}{$\begin{array}{l}\text { Temperature response-percentage maximum error caused by any } \\
\text { change of ambient temperature within an interval of } 50 \mathrm{~K}\end{array}$} & 1 & 2 \\
\hline \multicolumn{2}{|c|}{$\begin{array}{l}\text { Nonlinearity-percentage deviation from the responsivity at } 500 \mathrm{~W} / \mathrm{m}^{2} \\
\text { caused by any change of irradiance within the range of } 100-1,100 \\
\mathrm{~W} / \mathrm{m}^{2}\end{array}$} & 0.2 & 0.5 \\
\hline \multicolumn{2}{|c|}{$\begin{array}{l}\text { Spectral sensitivity-percentage deviation of the product of spectral } \\
\text { absorptance and spectral transmittance from the corresponding mean } \\
\text { within the range of } 300-3,000 \mathrm{~nm}\end{array}$} & 0.5 & 1.0 \\
\hline \multicolumn{2}{|c|}{$\begin{array}{l}\text { Tilt response-percentage deviation from the responsivity at } 0^{\circ} \text { tilt } \\
\text { (horizontal) caused by a change in tilt from } 0^{\circ}-90^{\circ} \text { at } 1,000 \mathrm{~W} / \mathrm{m}^{2}\end{array}$} & 0.2 & 0.5 \\
\hline \multicolumn{4}{|c|}{ Achievable uncertainty ( $95 \%$ confidence level): } \\
\hline 1-minute totals & $\begin{array}{l}\text { Percent } \\
\mathrm{kJ} / \mathrm{m}^{2} \\
\mathrm{Wh} / \mathrm{m}^{2}\end{array}$ & $\begin{array}{l}0.9 \\
0.56 \\
0.16 \\
\end{array}$ & $\begin{array}{l}1.8 \\
1 \\
0.28 \\
\end{array}$ \\
\hline 1-hour totals & $\begin{array}{l}\text { Percent } \\
\mathrm{kJ} / \mathrm{m}^{2} \\
\mathrm{Wh} / \mathrm{m}^{2}\end{array}$ & $\begin{array}{l}0.7 \\
21 \\
5.83\end{array}$ & $\begin{array}{l}1.5 \\
54 \\
15.0\end{array}$ \\
\hline Daily totals & $\begin{array}{l}\text { Percent } \\
\mathrm{kJ} / \mathrm{m}^{2} \\
\mathrm{Wh} / \mathrm{m}^{2}\end{array}$ & $\begin{array}{l}0.5 \\
200 \\
55.6\end{array}$ & $\begin{array}{l}1 \\
400 \\
111.1\end{array}$ \\
\hline
\end{tabular}

${ }^{a}$ High quality means "near state of the art"; good quality refers to instruments for network operation. 
Table 3-3. WMO Characteristics of Operational Pyranometers used to measure GHI

\begin{tabular}{|c|c|c|c|}
\hline Characteristic & $\begin{array}{l}\text { High } \\
\text { Quality }\end{array}$ & $\begin{array}{l}\text { Good } \\
\text { Quality }\end{array}$ & $\begin{array}{c}\text { Moderate } \\
\text { Quality }\end{array}$ \\
\hline Response time-95\% response & $<15 \mathrm{~s}$ & $<30 \mathrm{~s}$ & $<60 \mathrm{~s}$ \\
\hline $\begin{array}{l}\text { Zero offset-Response to } 200 \mathrm{~W} / \mathrm{m}^{2} \text { net thermal } \\
\text { radiation (ventilated); response to } 5-\mathrm{K} / \mathrm{h} \text { change in } \\
\text { ambient temperature }\end{array}$ & $\begin{array}{l}7 \mathrm{~W} / \mathrm{m}^{2} \\
2 \mathrm{~W} / \mathrm{m}^{2}\end{array}$ & $\begin{array}{l}15 \mathrm{~W} / \mathrm{m}^{2} \\
4 \mathrm{~W} / \mathrm{m}^{2}\end{array}$ & $\begin{array}{l}30 \mathrm{~W} / \mathrm{m}^{2} \\
8 \mathrm{~W} / \mathrm{m}^{2}\end{array}$ \\
\hline Resolution-smallest detectable change & $1 \mathrm{~W} / \mathrm{m}^{2}$ & $5 \mathrm{~W} / \mathrm{m}^{2}$ & $10 \mathrm{~W} / \mathrm{m}^{2}$ \\
\hline Stability_change per year, percentage of full scale & 0.8 & 1.5 & 3.0 \\
\hline $\begin{array}{l}\text { Directional response for beam radiation-the range of } \\
\text { errors caused by assuming that the normal incidence } \\
R s \text { is valid for all directions when measuring, from any } \\
\text { direction, a beam radiation that has a normal incidence } \\
\text { irradiance of } 1,000 \mathrm{~W} / \mathrm{m}^{2}\end{array}$ & $10 \mathrm{~W} / \mathrm{m}^{2}$ & $20 \mathrm{~W} / \mathrm{m}^{2}$ & $30 \mathrm{~W} / \mathrm{m}^{2}$ \\
\hline $\begin{array}{l}\text { Temperature response-percentage maximum error } \\
\text { caused by any change of ambient temperature within } \\
\text { an interval of } 50 \mathrm{~K}\end{array}$ & 2 & 4 & 8 \\
\hline $\begin{array}{l}\text { Nonlinearity-percentage deviation from the } R s \text { at } 500 \\
\mathrm{~W} / \mathrm{m}^{2} \text { caused by any change of irradiance within the } \\
\text { range from } 100-1,000 \mathrm{~W} / \mathrm{m}^{2}\end{array}$ & 0.5 & 1 & 3 \\
\hline $\begin{array}{l}\text { Spectral sensitivity-percentage deviation of the } \\
\text { product of spectral absorptance and spectral } \\
\text { transmittance from the corresponding mean within the } \\
\text { range from } 300-3,000 \mathrm{~nm}\end{array}$ & 2 & 5 & 10 \\
\hline $\begin{array}{l}\text { Tilt response-percentage deviation from the } R s \text { at } \\
0^{\circ} \text { tilt (horizontal) caused by a change in tilt from } 0^{\circ}- \\
90^{\circ} \text { at } 1,000 \mathrm{~W} / \mathrm{m}^{2}\end{array}$ & 0.5 & 2 & 5 \\
\hline $\begin{array}{l}\text { Achievable uncertainty-95\% confidence level } \\
\text { Hourly totals } \\
\text { Daily totals }\end{array}$ & $\begin{array}{l}3 \% \\
2 \% \\
\end{array}$ & $\begin{array}{l}8 \% \\
5 \% \\
\end{array}$ & $\begin{array}{l}20 \% \\
10 \% \\
\end{array}$ \\
\hline
\end{tabular}


Table 3-4. ISO 9060 Specifications Summary for Pyrheliometers Used To Measure DNI

\begin{tabular}{|c|c|c|c|}
\hline \multicolumn{4}{|c|}{ Pyrheliometer Specification List } \\
\hline \multirow[b]{2}{*}{ Specification } & \multicolumn{3}{|c|}{ Class of Pyrheliometer } \\
\hline & $\begin{array}{c}\text { Secondary } \\
\text { Standard Class }\end{array}$ & First Class & $\begin{array}{l}\text { Second } \\
\text { Class }\end{array}$ \\
\hline Response time- $-95 \%$ response & $<15 \mathrm{~s}$ & $<20 \mathrm{~s}$ & $<30 \mathrm{~s}$ \\
\hline $\begin{array}{l}\text { Zero offset } \\
\text { Response to } 5-\mathrm{K} \mathrm{h}-1 \text { change in } \\
\text { ambient temperature }\end{array}$ & $\pm 1 \mathrm{Wm}^{-2}$ & $\pm 3 \mathrm{Wm}^{-2}$ & $\pm 6 \mathrm{Wm}^{-2}$ \\
\hline $\begin{array}{l}\text { Stability-percentage of full scale, } \\
\text { change/year }\end{array}$ & $\pm 0.5 \%$ & $\pm 1 \%$ & $\pm 2 \%$ \\
\hline $\begin{array}{l}\text { Nonlinearity-percentage deviation } \\
\text { from the responsivity at } 500 \mathrm{~W} / \mathrm{m}^{2} \\
\text { because of change in irradiance } \\
\text { between } 100 \mathrm{Wm}^{-2} \text { and } 1,000 \mathrm{Wm}\end{array}$ & $\pm 0.2 \%$ & $\pm 0.5 \%$ & $\pm 2 \%$ \\
\hline $\begin{array}{l}\text { Spectral selectivity-percentage } \\
\text { deviation of the product of the spectral } \\
\text { absorptance and the spectral } \\
\text { transmittance from the corresponding } \\
\text { mean between } 0.35 \mu \mathrm{m} \text { and } 1.5 \mu \mathrm{m}\end{array}$ & $\pm 0.5 \%$ & $\pm 1 \%$ & $\pm 5 \%$ \\
\hline $\begin{array}{l}\text { Temperature response-total } \\
\text { percentage deviation because of } \\
\text { change in ambient temperature within } \\
\text { an interval of } 50 \mathrm{~K}\end{array}$ & $\pm 1 \%$ & $\pm 2 \%$ & $\pm 10 \%$ \\
\hline $\begin{array}{l}\text { Tilt response-percentage deviation } \\
\text { from the responsivity at } 0^{\circ} \text { tilt } \\
\text { (horizontal) because of change in tilt } \\
\text { from } 0^{\circ}-90^{\circ} \text { at } 1,000 \mathrm{~W} / \mathrm{m}^{-2} \text { irradiance }\end{array}$ & $\pm 0.2 \%$ & $\pm 0.5 \%$ & $\pm 2 \%$ \\
\hline $\begin{array}{l}\text { Traceability-maintained by periodic } \\
\text { comparison }\end{array}$ & $\begin{array}{l}\text { With a primary } \\
\text { standard } \\
\text { pyrheliometer }\end{array}$ & $\begin{array}{l}\text { With a } \\
\text { secondary } \\
\text { standard } \\
\text { pyrheliometer }\end{array}$ & $\begin{array}{l}\text { With a first- } \\
\text { class } \\
\text { pyrheliometer } \\
\text { or better }\end{array}$ \\
\hline
\end{tabular}


Table 3-5. ISO 9060 Specifications Summary for Pyranometers

\begin{tabular}{|c|c|c|c|}
\hline \multicolumn{4}{|c|}{ Pyrheliometer Specification List } \\
\hline \multirow[b]{2}{*}{ Specification } & \multicolumn{3}{|c|}{ Class of Pyrheliometer $^{a}$} \\
\hline & $\begin{array}{c}\text { Secondary } \\
\text { Standard Class }\end{array}$ & First Class & $\begin{array}{l}\text { Second } \\
\text { Class }\end{array}$ \\
\hline Response time-95\% response & $<15 \mathrm{~s}$ & $<30 \mathrm{~s}$ & $<60 \mathrm{~s}$ \\
\hline $\begin{array}{l}\text { Zero offset } \\
\quad \text { Response to } 200 \mathrm{Wm}^{-2} \text { net thermal }\end{array}$ & $\pm 7 \mathrm{Wm}^{-2}$ & $\pm 15 \mathrm{Wm}^{-2}$ & $\pm 30 \mathrm{Wm}^{-2}$ \\
\hline $\begin{array}{l}\text { Response to } 5-\mathrm{Kh}^{-1} \text { change in } \\
\text { ambient temperature }\end{array}$ & $\pm 2 \mathrm{Wm}^{-2}$ & $\pm 4 \mathrm{Wm}^{-2}$ & $\pm 8 \mathrm{Wm}^{-2}$ \\
\hline $\begin{array}{l}\text { Stability-percentage change in } \\
\text { responsivity per year }\end{array}$ & $\pm 0.8 \%$ & $\pm 1.5 \%$ & $\pm 3 \%$ \\
\hline $\begin{array}{l}\text { Nonlinearity-percentage deviation } \\
\text { from the responsivity at } 500 \mathrm{~W} / \mathrm{m}^{2} \\
\text { because of change in irradiance } \\
\text { between } 100 \mathrm{Wm}^{-2} \text { and } 1,000 \mathrm{Wm}^{-2}\end{array}$ & $\pm 0.5 \%$ & $\pm 1 \%$ & $\pm 3 \%$ \\
\hline $\begin{array}{l}\text { Directional response for beam radiation } \\
\text { (the range of errors caused by } \\
\text { assuming that the normal incidence } \\
\text { responsivity is valid for all directions } \\
\text { when measuring, from any direction, a } \\
\text { beam radiation that has a normal } \\
\text { incidence irradiance of } 1,000 \mathrm{Wm}^{-2}\end{array}$ & $\pm 10 \mathrm{Wm}^{-2}$ & $\pm 20 \mathrm{Wm}^{-2}$ & $\pm 30 \mathrm{Wm}^{-2}$ \\
\hline $\begin{array}{l}\text { Spectral selectivity-percentage } \\
\text { deviation of the product of the spectral } \\
\text { absorptance and the spectral } \\
\text { transmittance from the corresponding } \\
\text { mean between } 0.35 \mu \mathrm{m} \text { and } 1.5 \mu \mathrm{m}\end{array}$ & $\pm 3 \%$ & $\pm 5 \%$ & $\pm 10 \%$ \\
\hline $\begin{array}{l}\text { Temperature response-total } \\
\text { percentage deviation because of } \\
\text { change in ambient temperature within } \\
\text { an interval of } 50 \mathrm{~K}\end{array}$ & $2 \%$ & $4 \%$ & $8 \%$ \\
\hline $\begin{array}{l}\text { Tilt response-percentage deviation } \\
\text { from the responsivity at } 0^{\circ} \text { tilt } \\
\text { (horizontal) because of change in tilt } \\
\text { from } 0^{\circ}-90^{\circ} \text { at } 1,000 \mathrm{~W} / \mathrm{m}^{-2} \text { irradiance }\end{array}$ & $\pm 0.5 \%$ & $\pm 2 \%$ & $\pm 5 \%$ \\
\hline
\end{tabular}

${ }^{\mathrm{a}}$ The highest category for pyranometers is the secondary standard because the most accurate determination of GHI has been shown to be the sum of the DNI measured by an absolute cavity radiometer and of the DHI measured by a secondary standard pyranometer shaded from the DNI by a disk.

\subsubsection{Pyrheliometer and Pyranometer Calibrations}

As stated above, the signal of field radiometers is a voltage or a current that is proportional to the solar irradiance reaching the detector. A calibration factor is required to convert the current or voltage to a solar irradiance. The calibration factor, $C_{c a l}$, is the inverse of the responsivity, $R s$. 
For example, the responsivity of a thermopile pyrheliometer is given in $\mu \mathrm{V}$ per $\mathrm{W} / \mathrm{m}^{2}$. The irradiance, $E$, can be obtained from the voltage signal, $V_{p y r}$, or from the instrument's responsivity as

$$
E=V_{p y r} / R s=V_{p y r} \cdot C_{c a l}
$$

These calibration constants are not temporally stable over long periods, as demonstrated by the time series plot of calibration responsivities of two pyrheliometers in Figure 3-12. The instability can be due to changes in the instrument, meteorological conditions at the time of calibration, stability of the calibration reference radiometer(s), performance of the data acquisition system, and other factors included in the estimated uncertainty of each calibration result.

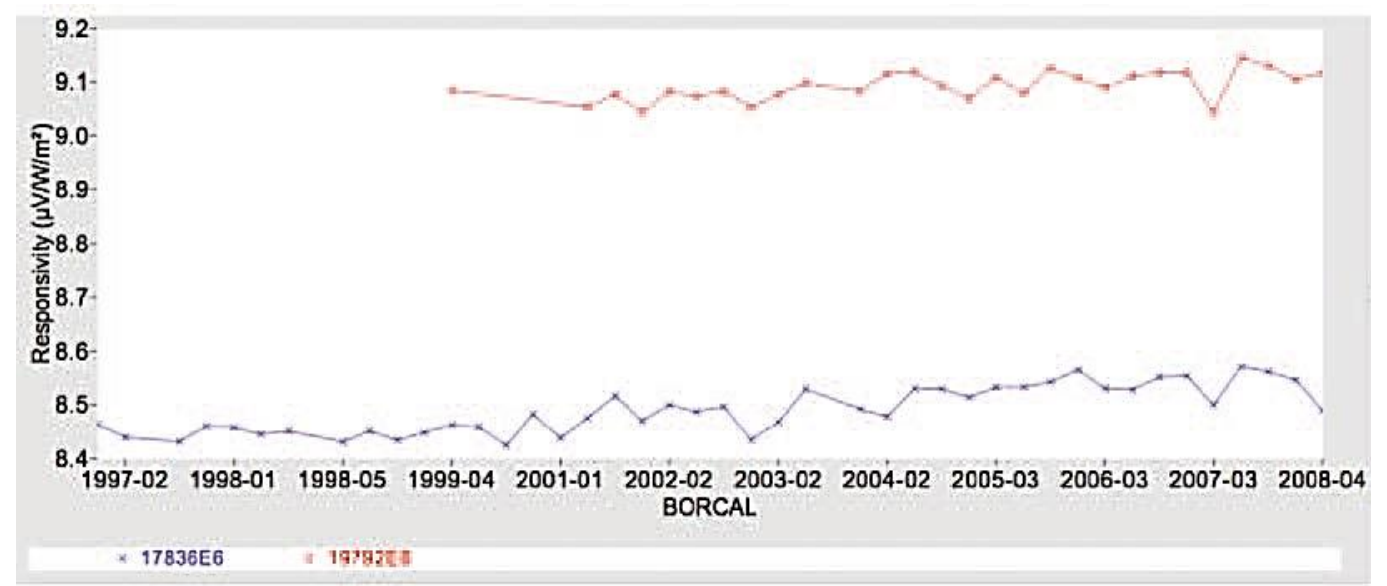

Figure 3-12. Calibration histories for two pyrheliometer control instruments spanning 12 years. Image by NREL

The calibration of pyrheliometers and pyranometers is described in detail in international standards ASTM G167-05, ASTM E816-05, ASTM E824-05, ASTM G183-05, ISO 9059, ISO 9846, and ISO 9847. The calibration methods described in ISO 9846 (ISO 1993) for pyranometers and in ISO 9059 (ISO 1990) for pyrheliometers are based on simultaneous solar irradiance measurements with test and reference instruments. ISO 9847 (ISO 1992) describes pyranometer calibrations using a reference pyranometer.

Pyrheliometers are calibrated following ISO 9059 by comparing the voltage signal of the tracked test pyrheliometer to the reference DNI from one or a group of reference pyrheliometers. For each simultaneous measurement pair, a preliminary responsivity can be calculated as the ratio of the test instrument's voltage and the reference DNI (Figure 3-13, right). After rejecting outliers and data collected during inappropriate conditions, an average responsivity can be determined. Because some pyrheliometers show a noticeable dependence on the solar zenith angle (SZA), specific angular responsivities can also be derived (Figure 3-13, left and bottom). For this calibration method, it is important that clouds do not mask the sun or the circumsolar region. The calibration can be affected if significant levels of circumsolar radiation prevail during the calibration. This risk increases with the instrument's FOV. Hence, Linke turbidities should be less than 6 according to the standard method. The Linke turbidity coefficient, $T_{L}$, is a measure of atmospheric attenuation under cloudless conditions. It represents the number of clean-and-dry 
atmospheres that would result in the same attenuation as the real cloudless atmosphere. One method to derive the Linke turbidity from DNI is presented in Ineichen and Perez (2002).

\section{Calibration Results \\ 19047E6 Eppley NIP}

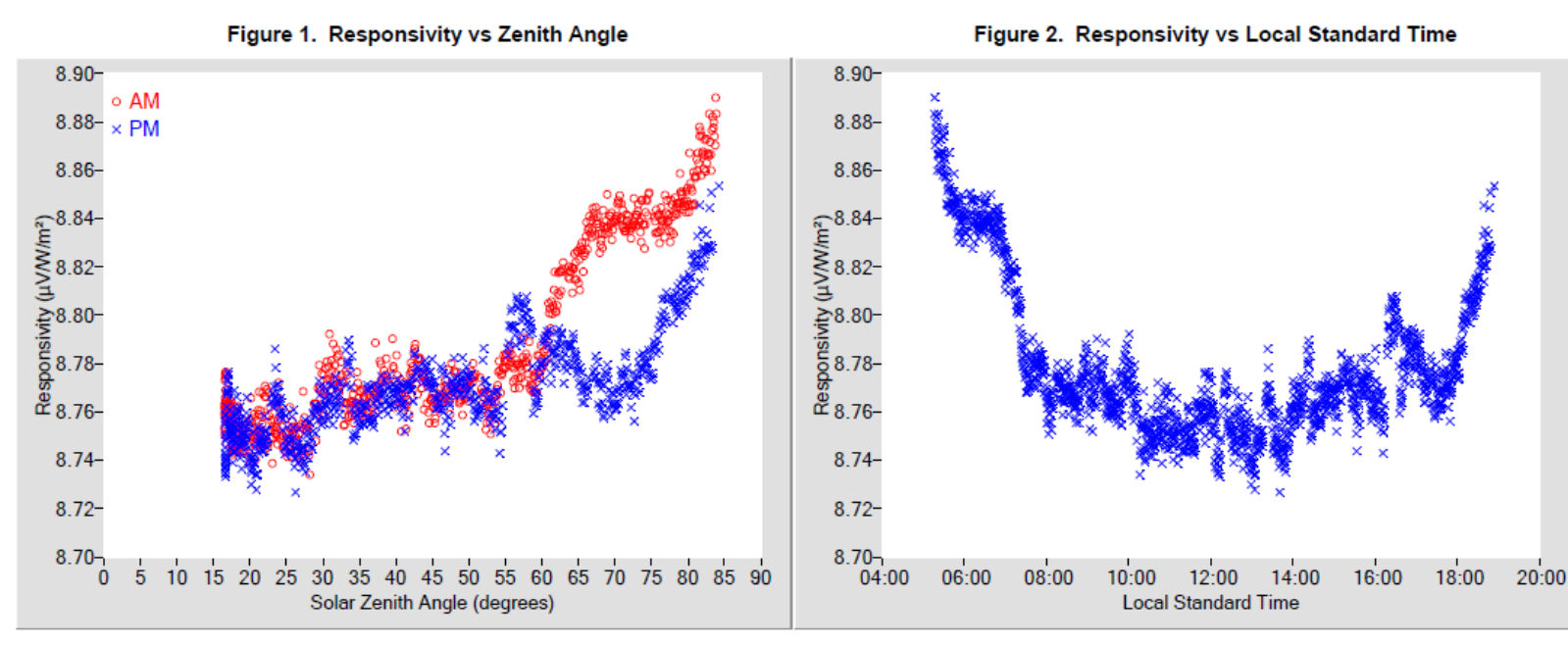

\begin{tabular}{|ccc|}
\hline \multicolumn{3}{|c|}{ Table 2. Calibration Label Values } \\
\hline RS @ $45^{\circ}\left(\mu \mathrm{V} / \mathrm{W} / \mathrm{m}^{2}\right) \ddagger$ & U95 $(\%) \dagger$ & Tilt / Azm \\
\hline 8.7678 & $+0.80 /-0.62$ & N/A \\
\hline \hline Valid zenith angle range: & $30.0^{\circ}$ to $60.0^{\circ}$ & \\
$\ddagger$ Estimated thermal offset error during calibration is unspecified. &
\end{tabular}

Figure 3-13. Pyrheliometer calibration results summarizing (left) Rs compared to SZA and (right) compared to local standard time. Image by NREL

As mentioned above, the WRR must be used as traceable reference for the calibration of all terrestrial broadband radiometers, as stipulated by the internationally accepted Système International (SI). This internationally recognized measurement reference is a detector-based standard maintained by a group of electrically self-calibrating absolute cavity pyrheliometers at the WRC by the Physical Meteorological Observatory in Davos, Switzerland. The present accepted inherent uncertainty in the WRR is $\pm 0.3 \%$ (Finsterle 2011). Even though all radiometer calibrations must be traceable to the WRR, it does not mean that all radiometers are calibrated directly against the WRR. The calibration chain from the WRR to a field instrument can have several steps. For example, reference ACRs are used as national and institutional standards, and these instruments are calibrated by comparison to the WRR during international pyrheliometer comparisons conducted by the WRC once every 5 years. Pyranometers calibrated against traceable WRR reference pyrheliometers make these pyranometer calibrations WRR-traceable.

Pyranometers can be calibrated outdoors with three different methods. One option, as described in ISO 9846, is to compare the DNI output from a reference pyrheliometer to that derived from the test pyranometer using the shade-unshade method. The successive voltages $V_{\text {unshade }}$ and $V_{\text {shade }}$ are proportional to GHI (unshaded) and DHI (shaded), respectively. Using the reference DNI and 
the relationship described in Section 2.4, the responsivity $R s$ of the pyranometer under test for one measurement sequence can be derived.

$$
R s=\left[\left(\mathrm{V}_{\text {unshade }}-\mathrm{V}_{\text {shade }}\right) / \cos (\mathrm{SZA})\right] / \mathrm{DNI}
$$

This method is described in more detail by Reda et al. (2003).

For this calibration method, virtually constant atmospheric conditions during the pair of shaded and unshaded measurements are required. Such conditions can only be found during clear-sky days. In addition to cloud cover, aerosol and water vapor variations might also affect the calibration. This explains why only data collected for a low $T_{L}$ (below 6) should be used for the calibration.

Another option offered by ISO 9846 consists of comparing the voltage signal of the test pyranometer obtained in the GHI measurement position to the GHI calculated from the DNI and DHI measurements of a reference pyrheliometer and a shaded reference pyranometer. The $R s$ of a pyranometer under calibration for one simultaneous set of three measurements can be computed from their unshaded signal $\left(\mathrm{V}_{\text {unshaded }}\right)$.

$$
R s=\mathrm{V}_{\text {unshaded }} /[\mathrm{DNI} \cdot \cos (\mathrm{SZA})+\mathrm{DHI}]
$$

Computing the $R s$ this way is called the component-summation calibration technique. Here again, $T_{L}$ should be below 6 and clear-sky conditions around the sun should exist during the whole calibration period.

The third option to calibrate pyranometers outdoors is described in ISO 9847. It compares a test pyranometer to a reference pyranometer while both sensors are in the same measurement position (either GHI or GTI). The $R s_{i}$ is then obtained as the ratio of the test signal to the reference irradiance. For outdoor pyranometer calibrations using a reference pyranometer (ISO 1992), the sky conditions are less precisely defined than for the other methods described above. The calibration interval is adjusted depending on sky conditions.

The indoor calibration methods from ISO 9847 use irradiance measurements under an artificial light source. For the first option, measurements are taken simultaneously after ensuring that the test and the reference pyranometer receive the same irradiance from an integrating sphere. This is done by switching pyranometer positions. The other option is to take consecutive measurements by mounting the test and the reference instrument one after the other in the same position under a direct beam. The indoor calibrations are carried out in a controlled environment that is independent from external meteorological conditions. However, if consecutive measurements are made over a long period, instabilities of the artificial light source increase the calibration uncertainty compared to outdoor calibrations. If simultaneous measurements are used, an additional uncertainty contribution comes from the fact that the test and the reference pyranometer can never receive exactly the same irradiance from the artificial light source. Furthermore, the incident angle of the radiation is usually not well defined for indoor calibrations. Because pyranometers deviate from true cosine response, this means that there is no exact one-to-one comparison between indoor calibrations and outdoor measurements. 
Therefore, in general, thorough outdoor calibrations with accurate reference instruments have lower uncertainties than indoor calibrations and thus are preferable.

\section{Calibration Results 31400F3 Eppley PSP}
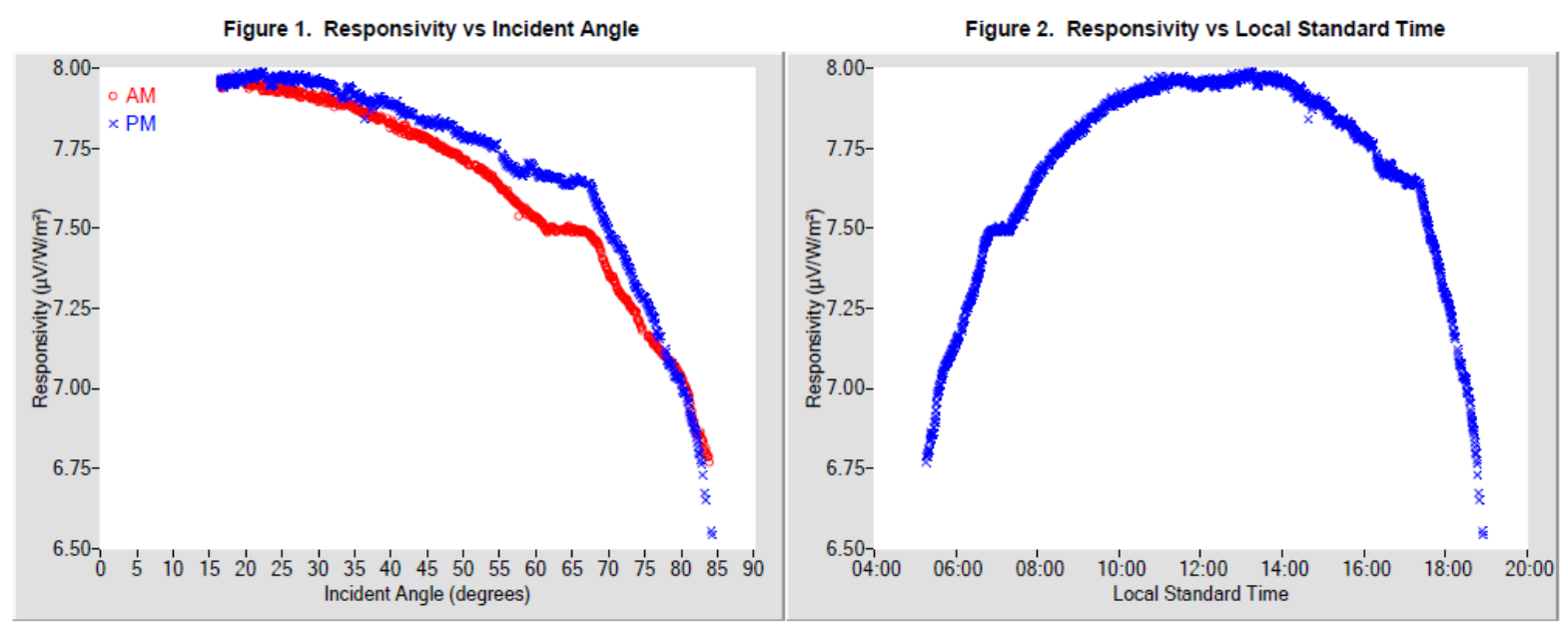

Table 2. Calibration Label Values
\begin{tabular}{|c|c|c|}
\hline RS @ $45^{\circ}\left(\mu \mathrm{V} / \mathrm{W} / \mathrm{m}^{2}\right) \ddagger$ & U95 $(\%) \dagger$ & Tilt $/$ Azm \\
\hline 7.8028 & $+2.61 /-4.08$ & $0.0^{\circ} / 0.0^{\circ}$ \\
\hline
\end{tabular}

† Valid incident angle range: $30.0^{\circ}$ to $60.0^{\circ}$

$\ddagger$ Estimated thermal offset error during calibration $=-20.000 \mathrm{~W} / \mathrm{m}^{2}$

Figure 3-14. Pyranometer calibration results summarizing Rs compared to (left) SZA and (right) local standard time. Image by DaryI Myers, NREL

The shade/unshade and component summation techniques, when conducted throughout a range of SZA, show that pyranometer responsivities are a function of it. The variation of $R s$ as a function of SZA is like a fingerprint or signature of each individual specimen of pyranometer (Figure 3-14), which means that the angular responsivities of different specimens of the same model can differ. Variations of pyranometer $R s$ can be symmetrical with respect to solar noon or highly skewed, depending on the mechanical alignment of the pyranometer, detector surface structure, and detector absorber material properties. Similar to what can be done in the case of pyrheliometer calibration, the pyranometer's $R s$ values can be combined into a function $R s=\mathrm{f}(\mathrm{SZA})$, representing the average responsivity for various incidence angle intervals. This function can be fitted to a polynomial curve to easily derive $R s$ as a function of the incidence angle. The best possible accuracy in GHI measurement results from using an SZA-dependent calibration factor for each individual measurement. This method, however, is only applicable to conditions with high DNI because the variation of responsivity with SZA is mostly caused by DNI and the associated cosine error. Another option to treat this incidence angle dependence is to determine a calibration factor for a given small incidence angle range and to apply correction factors depending on the angle. For situations when thick clouds mask the sun or for DHI measurements, no useful incidence angle can be found. In these cases, the angular distribution of the incoming irradiance cannot be approximated well by one incidence angle. For DHI measurements, it is recommended to use the $R s$ for a $45^{\circ}$ incidence angle. 
Accurate solid-state pyranometer calibration is not possible by simple application of these standards due to the uneven spectral response. A specific calibration method is discussed with that of Rotating Shadowband Irradiometer (RSI) instruments in Section 3.2.5.

\subsubsection{Correction Functions for Systematic Errors of Radiometers}

Some of the pyrheliometer and pyranometer measurement errors are systematic and can be reduced by applying correction functions. An example is the correction of the SZA or cosine response, as mentioned above. Some manufacturers provide one calibration constant for a pyranometer and additional correction factors for different intervals of SZA. This treatment of the incidence angle dependence is mathematically the same as using an incidence-angledependent responsivity.

Moreover, an additional temperature correction can be applied if the internal temperature of pyranometers or pyrheliometers is measured using a temperature-dependent resistor close to the sensor. Correction coefficients are often supplied by the manufacturer.

Measurements from only black (as opposed to black and white receivers) pyranometers can be corrected for the expected thermal offset using additional measurements from pyrgeometers (Figure 3-4, right). Pyrgeometers allow the determination of the downward longwave irradiance between approximately $4.5 \mu \mathrm{m}$ and $40 \mu \mathrm{m}$, based on their sensor (thermopile) signal and the instrument's body temperature. The thermopile is positioned below an opaque window that is only transparent to the specified infrared radiation wavelength range, while excluding all visible near and far infrared radiation. Most pyrgeometers must be positioned below a shading ball or disk to limit window heating by the DNI. Ventilation units are also used for pyrgeometers, just as in the case of pyranometers. If no pyrgeometer is available, a less accurate correction for the thermal offset can be made based on estimations of the thermal offset from the typically negative measurements collected during the night (Dutton et al. 2000).

Correction functions for photodiode pyranometers are presented in Section 3.2.5 on RSI.

\subsubsection{Systems for Determining Solar Irradiance Components}

A measurement system that independently measures the basic solar components, GHI, DNI, and DHI, will produce data with the lowest uncertainty if the instruments are properly installed and maintained. Alternatives exist to reduce the overall cost of such a system while offering potentially acceptable data accuracies. These alternatives are designed to eliminate the needs for a pyrheliometer mounted in an automatic solar tracker.

\subsubsection{Rotating Shadowband Irradiometers}

RSIs use a pyranometer that is periodically shaded by a motorized shadowband, which rapidly moves back and forth across the detector's FOV (Figure 3-15). The principle of operation of these RSIs is to measure GHI when unshaded and DHI when shaded. The DNI is calculated using the fundamental closure equation relating these three components:

$$
\mathrm{DNI}=(\mathrm{GHI}-\mathrm{DHI}) / \cos (\mathrm{SZA})
$$

RSIs are often called rotating shadowband radiometers (RSRs) or rotating shadowband pyranometers (RSPs), depending on the instrument manufacturer. RSI refers to all such 
instruments measuring irradiance by use of a rotating shadowband. There are two types of RSIs: RSIs with continuous rotation and RSIs with discontinuous rotation.
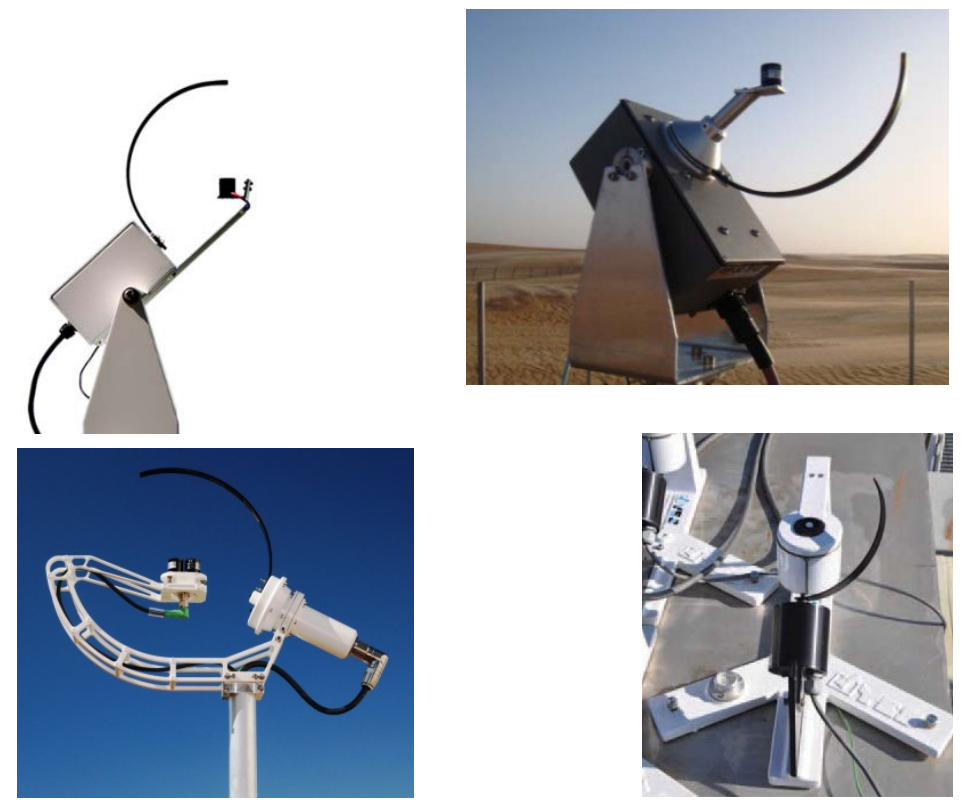

Figure 3-15. Four commercially available RSIs: (clockwise from upper left) Irradiance, Inc., model RSR2; Reichert GmbH RSP 4G (previously used by SM-AG); Yankee Environmental Systems, Inc., model SDR-1; and CSP-Services GmbH Twin-RSI. Photos by (clockwise from top left) Irradiance, Inc.; Reichert GmbH RSP 4G; NREL; and CSP-Services

The operational principle of RSIs with continuous rotation is shown in Figure 3-16. At the beginning of each rotation cycle, the shadowband is below the pyranometer in its rest position. The rotation is performed with constant angular velocity and takes approximately 1 second. During the rotation, the irradiance is measured with a high and constant sampling rate (approximately $1 \mathrm{kHz}$ ). This measurement is called a burst or sweep. At the beginning of the rotation, the pyranometer measures GHI. In the moment when the center of the shadow falls on the center of the sensor, it approximately detects DHI; however, the shadowband covers some portion of the sky, so that the minimum of the burst is actually less than DHI. Thus, so-called shoulder values are determined by curve analysis algorithms. Such algorithms are usually implemented in the data logger program and use the maximum of the absolute value of the burst's slope to find the position of the "shoulder values." The difference between GHI and the average of the two shoulder values is added to the minimum of the curve to obtain the "actual" DHI. Subsequently, DNI is calculated by the data logger using GHI, DHI, and the actual SZA calculated by the known time and coordinates of the location, as stated above. All of the RSIs shown in Figure 3-15 (except for the SDR-1 model) work with a continuous rotation. 


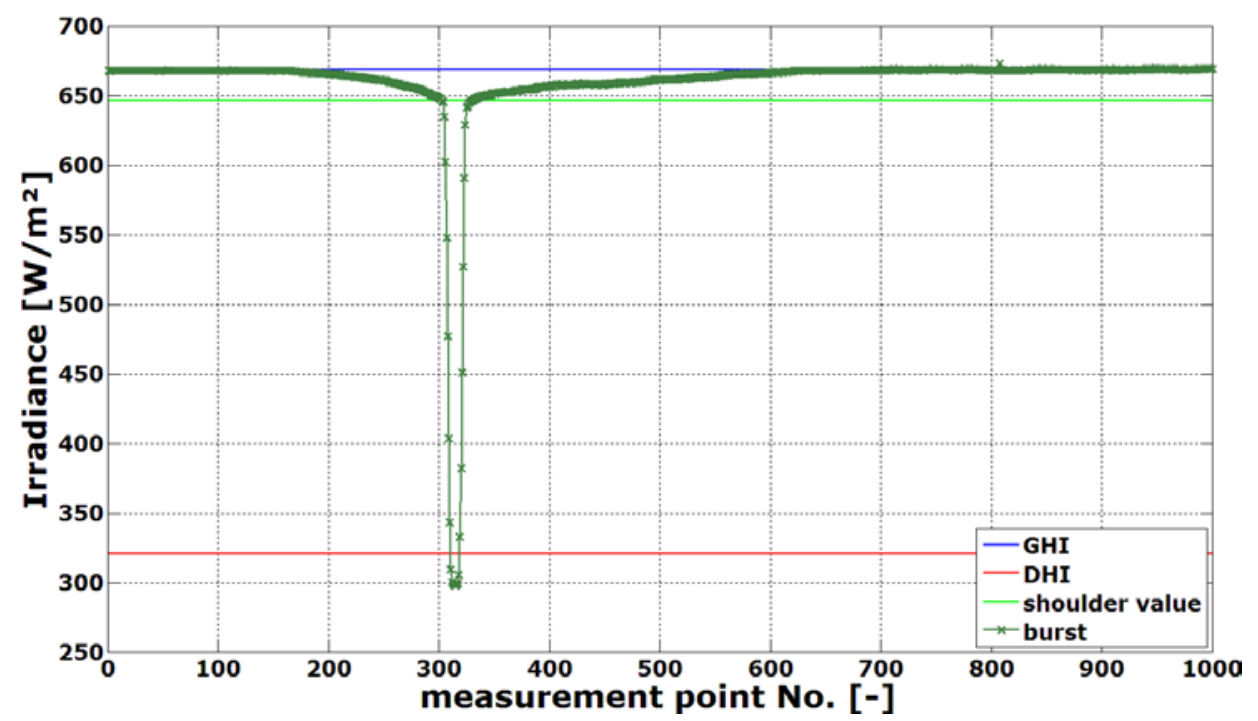

Figure 3-16. Burst (sweep) with sensor signal and the derived GHI, shoulder values, and DHI. Image from Wilbert (2014)

RSIs with discontinuous rotation do not measure the complete burst - only four points of it. First, the GHI is measured while the shadowband is in the rest position. Then the shadowband rotates from the rest position toward the position at which it nearly shades the diffuser, stops, and a measurement is taken (e.g., during 1 second for the SDR-1 in Figure 3-15). Then it continues the rotation toward the position at which the shadow lies centered on the diffuser, and another measurement is taken. The last point is measured in a position at which the shadow just passed the diffuser. The measurement with the completely shaded diffuser is used equivalently to the minimum of the burst, as shown in Figure 3-16. The two measurements for which the shadow is close to the diffuser are used equivalently to the shoulder values to correct for the portion of the sky blocked by the shadowband.

The two types of RSIs just described have advantages and disadvantages. An RSI with continuous rotation needs a detector with a fast response time (much less than 1 second; e.g., approximately $10 \mu \mathrm{s}$ ). Because thermopile sensors cannot be used, semiconductor sensors are used instead - most likely using Si. An example is the Si-based radiometer model LI-200SA shown in Figure 3-11. Because of the nonhomogeneous spectral response of such Si sensors (see Figure 3-2), the measurement accuracy of secondary-standard thermopile pyranometers cannot be reached. Correction functions for this and other systematic errors have to be applied to reach the accuracy required in resource assessments, albeit still not on par with the accuracy of thermopile instruments. These correction functions are discussed below.

RSIs with discontinuous rotation can use sufficiently long measurement times for each of the four points to allow the use of a thermopile detector (e.g., the Yankee TSR-1 thermopile shadowband radiometer). Thus, the spectral error of a Si detector can be avoided — at least partly. So far, RSIs with discontinuous rotation typically rely on a diffuser, which has its own uneven spectral transmittance over the shortwave spectrum. Hence, the spectral error of such RSIs cannot be neglected. Furthermore, the discontinuous rotation is connected to other disadvantages compared to the continuous rotation. While RSIs with continuous rotation are not affected by small azimuth alignment errors (within approximately $\pm 5^{\circ}$ ), the azimuth alignment of RSIs with 
discontinuous rotation is crucial for their accuracy. Moreover, the accuracy of the sensor's coordinates and sweep time is more important for the discontinuous rotation. If the shadowband stops at the wrong position, the DHI measurement is incorrect. Further, the duration of the measurement with a discontinuous rotation increases the measurement uncertainty. This is especially relevant if the RSI uses a thermopile sensor and if sky conditions are not stable (e.g., cloud passages). If GHI and the sky radiance distribution change during the four-point measurement, the data used to determine DHI will deviate noticeably from the true value. In contrast, this complication is not relevant in the case of continuously rotating RSIs because their rotation takes approximately only 1 second.

DHI is typically determined one or four times per minute, but GHI measurements can be sampled at a higher frequency whenever the shadowband does not rotate-for example, every second. The temporal variation of GHI also contains some information about any concomitant change in DNI. Different algorithms are used to determine the averages of DHI and DNI between two DHI measurements using the more frequent GHI measurements.

The initially lower accuracy of RSIs compared to ISO 9060 first-class pyrheliometers and secondary-standard pyranometers is often compensated by some unique advantages of RSIs. Their low soiling susceptibility (Pape et al. 2009; Geuder and Quaschning 2006; Maxwell et al. 1999), low power demand, and comparatively lower cost (instrumentation and O\&M), provide significant advantages compared to thermopile sensors, at least when operated under the measurement conditions of remote weather stations, where power and daily maintenance requirements constitute key (and potentially very costly) issues.

Without application of any correction of the systematic deviations and a matched calibration method, RSIs yield only an uncertainty of $5 \%-10 \%$ under the best circumstances. This accuracy is notably improved, to $\approx 2 \%-3 \%$, with proper calibration and application of advanced correction functions (Wilbert et al. 2016), which are described in what follows. Most instrument providers also offer post-processing software or services that include these correction functions. Users should ask the manufacturer whether such post-processing is part of the instrument package and is readily available.

Because of the stated disadvantages of RSIs with discontinuous rotation and the higher relevance of RSIs with continuous rotation for solar energy applications, the focus is placed on RSIs with Si detectors and continuous rotation in the following. More information about RSIs with discontinuous rotation can be found in Harrison et al. (1994). Further general information on the accuracy of RSIs can be found in Chapter 6.

\subsubsection{Correction Functions for RS/s}

The main systematic errors of RSIs with $\mathrm{Si}$ sensors are caused by the spectral response of the $\mathrm{Si}$ detector, its cosine response, and its temperature dependence.

Several research groups have developed correction functions that reduce systematic errors in RSI readings. In all cases, the Si detector of the RSI is a LICOR LI-200SA. Whereas temperature correction is similar in all versions (King and Myers 1997; Geuder et al. 2008), the methods for the spectral and cosine corrections vary among these authors. 
Alados et al. (1995) used tabular factors for different sky clearness and skylight brightness parameters as well as a functional correction depending on SZA. King and Myers (1997) proposed functional corrections in dependence on air mass (AM) and SZA, primarily targeting GHI. This approach was further developed by Augustyn et al. (2002) and Vignola (2006), including diffuse and subsequently direct beam irradiance. The combination of the GHI correction of Augustyn et al. (2002) and of the diffuse correction from Vignola (2006) provides a complete set of corrections for LI-200SA-based RSIs. Independently, a method for DNI, GHI, and DHI correction was developed by the German Aerospace Agency, Deutsches Zentrum für Luft- und Raumfahrt (DLR), using functional corrections that include a particular spectral parameter obtained from GHI, DHI, and DNI (Geuder et al. 2008). Additional corrections in dependence on AM and SZA were used. Another set of correction functions was later presented in Geuder et al. (2011). Further new correction methods are on their way (Vignola et al. 2016a, 2016b).

\subsubsection{Calibration Methods for RS/s}

In addition to the corrections above, special calibration techniques are required for RSIs. As of this writing, RSIs with continuous rotation are equipped with LI-200SA Si detectors. They usually come with pre-calibration values from the manufacturer (LI-COR) for GHI, based on outdoor comparisons with an Eppley pyranometer (PSP) with an accuracy stated as better than 5\% (LI-COR Biosciences 2005). Considering that the PSP has only limited performance (e.g., Gueymard and Myers 2009), an additional calibration (e.g., onsite or with respect to DHI, DNI, or GHI independently) of the RSIs can noticeably improve their accuracy (Wilbert et al. 2016).

Because of the rather narrow and inhomogeneous spectral response of the photodiodes and the combined measurement of DHI and GHI, only some aspects of the existing ISO standards for pyrheliometer and pyranometer calibration can be transferred to RSI calibration. Calibrating RSI instruments involves field calibrating them for DNI, DHI, and GHI independently. Each of these three steps is challenging because each irradiance component has a distinct spectral composition that can change over the day or from location to location. Because of the spectral response of the $\mathrm{Si}$ detectors and/or diffusers, it is problematic to calibrate an RSI based on only a few series of measurements and under the special conditions defined in ISO 9847 and ISO 9059. This is possible for thermopile sensors because of their homogenous spectral response covering at least $300-3,000 \mathrm{~nm}$ (which amounts to $>99 \%$ of the ASTM G173 DNI spectrum). A similar calibration method of RSIs would need the additional — but incorrect — assumption that all RSIs from a single manufacturer have exactly the same nominal spectral and cosine response, which would then be well described by nominal correction functions. Hence, using separate calibration constants for at least two of the three components (GHI, DHI, and DNI) is recommended. However, some RSI calibration methods purport only to GHI calibration. The current best practice is to consider a sufficiently long calibration period, so as to include a wide variety of meteorological conditions, conducive to a large range of distributions in the incident spectrum. Such conditions should be assessed and characterized wisely during the calibration process. The calibration accuracy generally improves when the atmospheric conditions during the calibration closely represent those at the site where the RSI is intended to be operated later. In addition to cloud cover, the effects of aerosols, water vapor, and site altitude on the solar spectrum have to be considered (Myers 2011; Wilbert et al. 2016). Calibrations with artificial radiation sources that lack the spectral power distributions of natural solar radiation components usually also lack 
the variety of natural irradiation conditions; therefore, field calibrations under natural irradiation conditions should yield more accurate calibrations and are thus preferable.

Outdoor RSI calibrations are performed at a small number of laboratories in the world, such as the National Renewable Energy Laboratory (NREL) in Golden, Colorado, and DLR at the Plataforma Solar de Almería (PSA) in Spain. Additionally, onsite calibrations are performed by a small number of specialized companies. At PSA, for instance, RSIs are operated parallel to ISO9060 first-class pyrheliometers and secondary-standard pyranometers under real-sky conditions (Figure 3-17). The duration of RSI calibrations is from several hours up to more than one year. These longer calibration periods provide a database for the analysis of systematic signal deviations and measurement accuracy. An analysis of the dependence of the calibration constants on the duration of the calibration period, as well as more details on two possible calibration methods, are presented in Jessen et al. (2016) and Geuder et al. (2012). Data quality is analyzed and compared to the reference irradiances. RSI calibrations are performed according to the different methods described above. All published calibration techniques are based on the comparison of corrected RSI signals (using the existing correction functions described above) to reference irradiance measurements obtained with thermopile sensors.

Depending on the calibration method, one, two, or even three calibration constants are defined. The motivation for determining one calibration constant is that only one pyranometer is used and that the calibration based on GHI is less time consuming than separate calibrations for GHI, DHI, and DNI. Because of the Si detector's spectral response, the spectral sensitivities for DHI, GHI, and DNI are not the same. Hence, the application of two or three calibration constants is physically reasonable, even though only one sensor is used.

Examples of drift in the GHI calibration constants obtained from Geuder et al. (2008) were later investigated for nine sensors in Geuder et al. (2010) and Jessen et al. (2016). For recalibration periods between 2 years and 3.75 years, changes in this GHI calibration constant were below 1\% in most cases. Recalibration is recommended at least every 2 years. An overview of current RSI calibration methods is presented in Jessen et al. (2016), and more details can be found in Geuder et al. $(2008,2010)$ and Kern (2010). A case study for the accuracy achievable by different combinations of correction functions and calibration methods is summarized in Chapter 6 .

The calibration techniques for RSIs can partially be used for other solid-state radiometers. Further details on RSIs and RSI-specific measurement best practices can be found in Wilbert et al. (2015). 


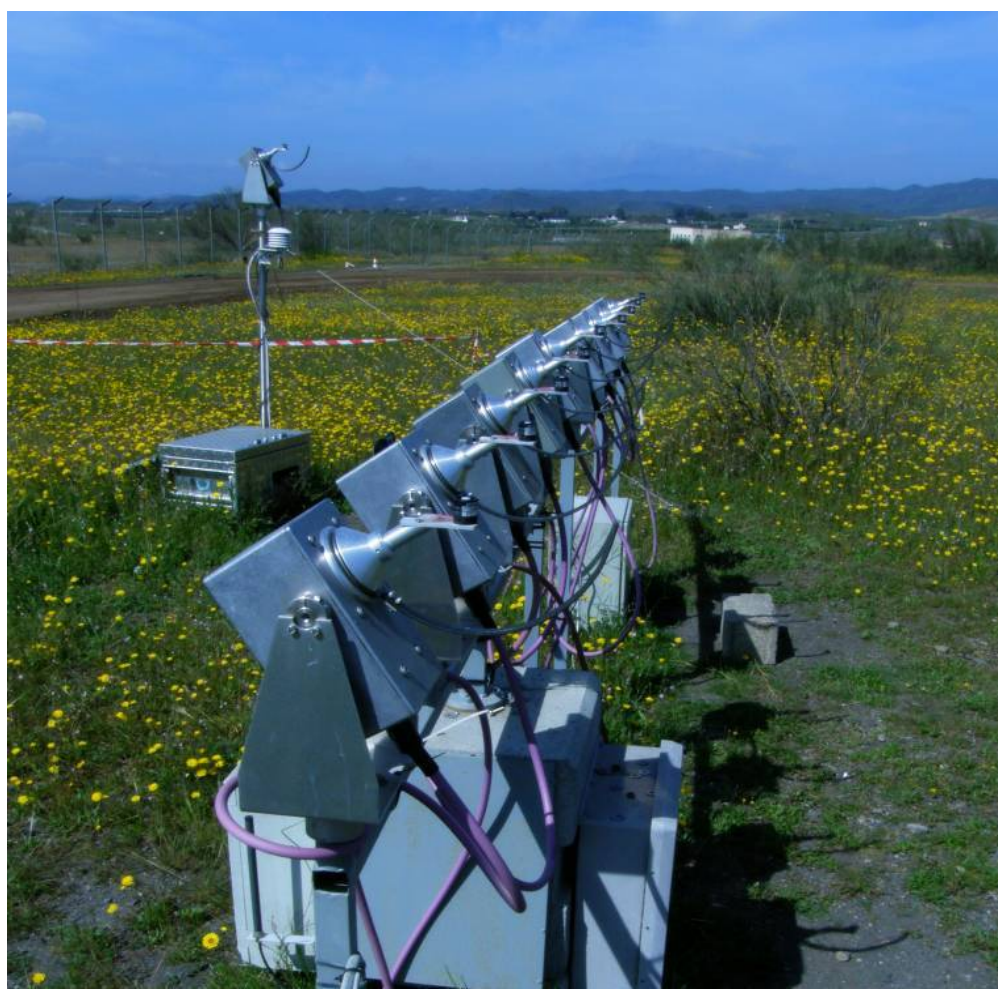

Figure 3-17. RSI calibration station at Plataforma Solar de Almería. Photo from Stefan Wilbert, DLR

\subsubsection{Other Instruments Used to Derive DHI and DNI}

In addition to the radiometers described above, there are other instruments that can be used to derive DHI or DNI from irradiance measurements. For example, the Scanning Pyrheliometer/Pyranometer (SCAPP) (Bergholter and Dehne 1994) or the sunshine duration sensor Soni e3 (Lindner 1984) can be used to derive DNI. These two sensors, however, only reach lower accuracies when compared to tracked pyrheliometers, thermopile pyranometers with shading balls, or even RSIs, as documented in Geuder et al. (2006). As an historical note, it should also be mentioned that researchers have developed methods for estimating daily integrated values of DNI from the vast archive of measurements from Campbell-Stokes sunshine recorders (Stanhill 1998; Painter 1981).

Recently, the EKO MS-05 instrument (Figure 3-18) has been introduced, based on an earlier sunshine recorder sensor (MS-093). The revised design uses a rotating mirror within a fixed glass tube tilted to latitude $\left(-58^{\circ}-+58^{\circ}\right)$. The mirror reflects the direct beam onto a broadband pyroelectric detector that senses DNI four times per minute. Preliminary tests have been conducted against a reference pyrheliometer (EKO MS-57) during the North American Pyrheliometer Comparison held at NREL during September 2016. The tests show satisfying results for a simple non-tracking instrument when DNI exceeds $600 \mathrm{~W} / \mathrm{m}^{2}$ (Figure 3-19). 


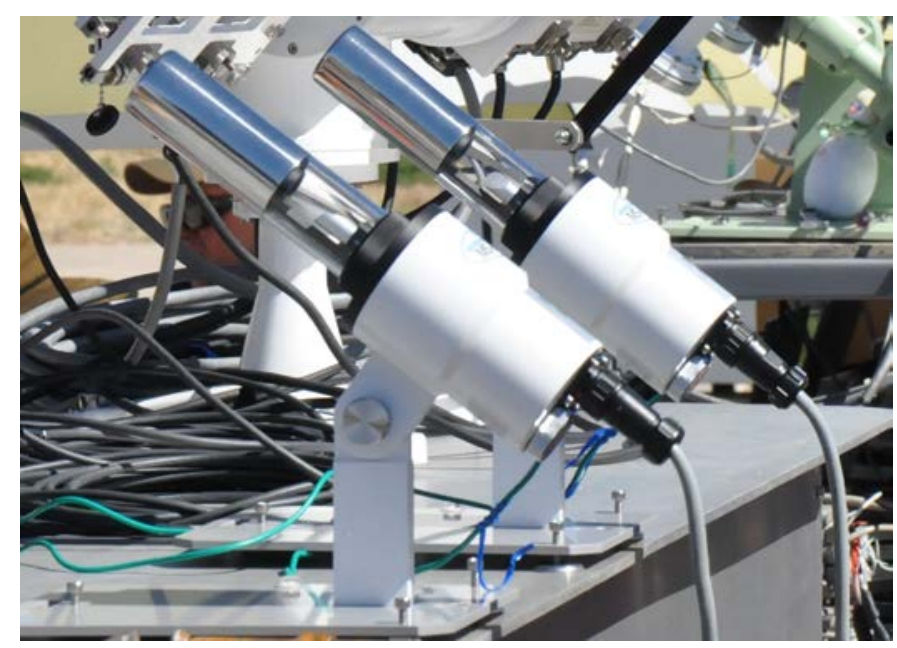

Figure 3-18. EKO MS-05. Image by NREL.

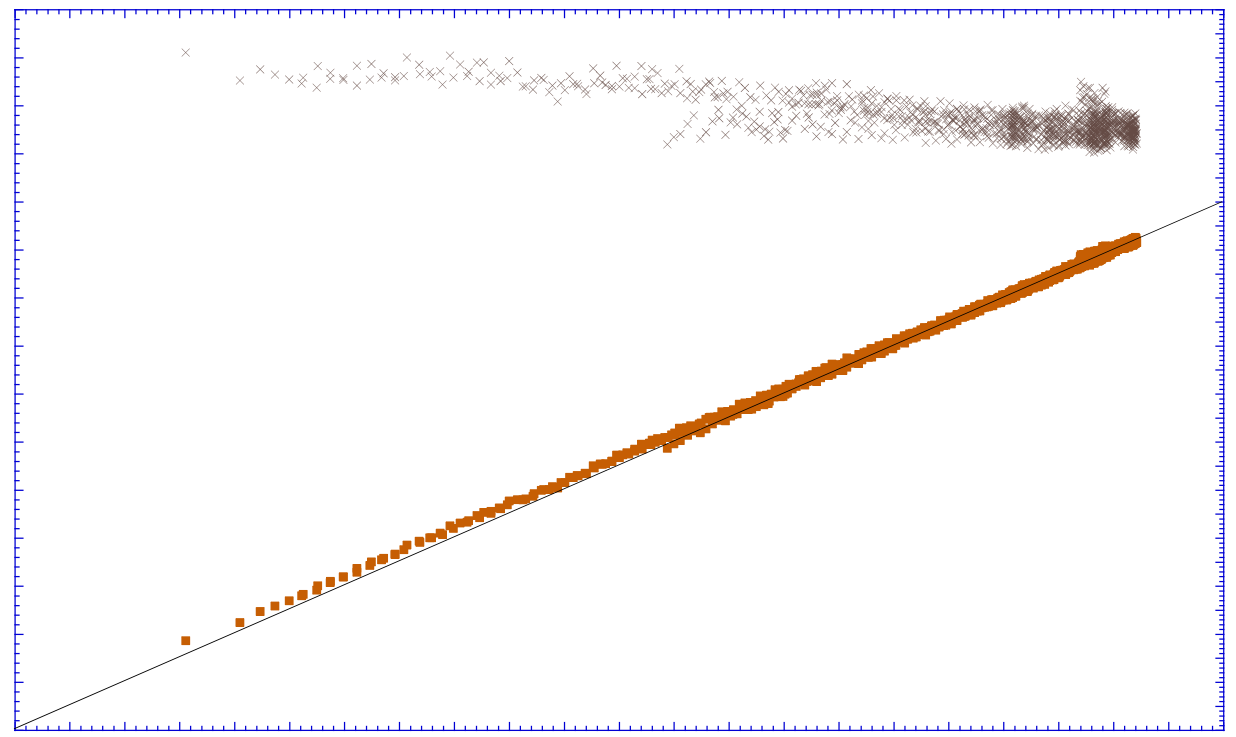

Figure 3-19. Comparison between 1-minute DNI measurements obtained with an EKO MS-05 and an MS-57 reference pyrheliometer at NREL during a 2-day test period

Recently, all-sky imagers have also been used to measure solar irradiance. The accuracy of such measurements is still too low for their application in resource assessment but of interest for forecasting applications. Hence, these instruments are discussed in Chapter 8 on solar forecasting.

Another option for estimating DNI from measurements of both DHI and GHI by a single instrument is the SPN1 (Figure 3-20). 


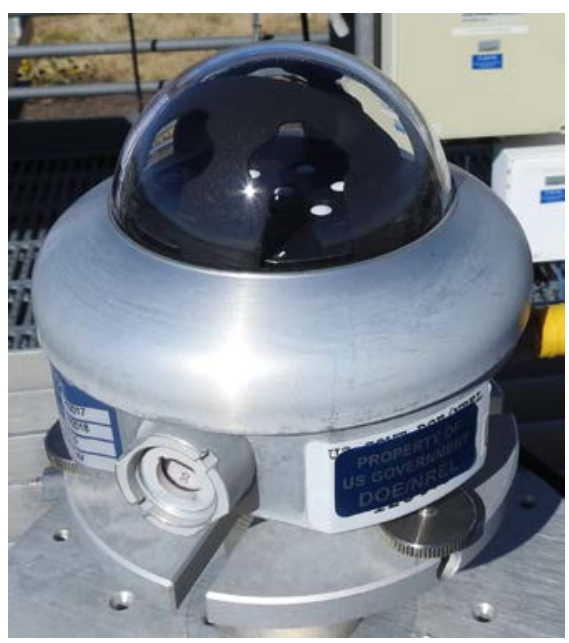

Figure 3-20. Delta-T Devices, Ltd., SPN1. Image by NREL.

The SPN1 is an instrument that consists of an array of seven fast-response thermopile radiation detectors that are distributed in a hexagonal pattern under a glass dome. The detectors are positioned under diffuser disks and a special hemispherical shadow mask. The shape of the mask is selected such that for any position of the sun in the sky there will always be one or more detectors that are fully shaded from the sun and exposed to approximately half of the diffuse radiance (for completely overcast skies). Also, one or more detectors are exposed to the full solar beam for all positions. The minimum and the maximum readings of the seven detectors are used to derive GHI and DHI.

With this principle of operation, GHI, DHI, and DNI can be derived without any moving parts and without needing alignment other than horizontal leveling. Further, the SPN1's low power demand (the temperature-controlled dome prevents dew and frost) increases its suitability for operation in remote sites compared to DNI or DHI measurements involving solar trackers. Test results indicate that the accuracy of the SPN1's GHI is comparable with RSIs, but its DNI and DHI readings have higher errors compared to the DNI measured with RSIs (Vuilleumier et al. 2012). Further, SPN1 performance results obtained at six different locations worldwide can be found in Badosa et al. (2015). An additional comparison with traditional radiometers is presented by Habte et al. (2016). The sensitivity of the SPN1 to sensor soiling is currently being investigated, as well as the possibility of improving its accuracy with more sophisticated corrections. The manufacturer publishes a guide to the SPN1's use, calibration, and expected accuracy (Wood 2015).

\subsection{Measuring Relevant Atmospheric Properties}

Several methods for estimating solar resources from available meteorological observations and satellite remote-sensing data have been and continue to be developed to address the absence of ground-based solar irradiance measurements (Myers 2013). In this section, we will describe the importance of atmospheric aerosols on the amount of DNI reaching the surface. Other atmospheric properties, such as total precipitable water vapor, total column ozone, and cloud amount, and standard meteorological observations, such as horizontal visibility, surface temperature, relative humidity, and wind conditions, are also useful for interpreting the quality of solar resource data. 
Measurements from sun photometers are used to determine aerosol optical properties such as spectral aerosol optical depth (AOD), single scattering albedo, asymmetry factor, or scattering phase functions. The most important result of these measurements for solar energy applications is the spectral AOD. One type of sun photometer measures spectral DNI and the spectral sky radiance at several different wavelengths (Figure 3-21). Other sun photometers only measure spectral DNI. Both types of sun photometers consist of one or more photodetectors positioned behind spectral filters and a collimator system. Additional polarization filters are optionally used. Solid-state sensors, such as photodiodes, are used for signal detection. A programmed tracking system is needed that directs the sun photometer to the selected sky coordinates. For those sun photometers that only measure spectral DNI, such a tracker can be the same as those used for pyrheliometers. For sky radiance measurements, special tracking systems are used. A spectral version of RSI called a multi-filter rotating shadowband radiometer can be used to derive both broadband and spectral DNI without a tracker. Because the above-mentioned aerosol properties are highly wavelength dependent, it is necessary to make measurements at more than a single wavelength. The energetically most relevant part of the solar spectrum from the UV to $\approx 1,600$ $\mathrm{nm}$ is covered by $4-10$ filters in most sun photometers.

Spectral DNI measurements are obtained by directing the sun photometer to the sun, just as in the case of a pyrheliometer. Sky radiance observations alternatively consist in either almucantar or principal plane measurements. A principal plane measurement is a series of sky radiance measurements with changing instrument zenith angle at the current solar azimuth angle. Conversely, an almucantar measurement results from a series of sky radiance measurements with varying instrumental azimuth angles at a fixed solar zenith angle. The direct-sun irradiance measurements are used to derive basic information on aerosols. First, the total optical depth is calculated. Then AOD is determined by subtracting the optical depths of all other atmospheric constituents such as molecules, water vapor, ozone, or nitrogen dioxide. While most of these optical depths are obtained from other sources (e.g., satellite retrievals or atmospheric models), sun photometers also include a water vapor channel whose central wavelength $(\approx 940 \mathrm{~nm})$ lies in a strong water vapor absorption band. The Ångström exponent can then be derived by fitting the spectral AOD data to the equation describing Ångström's law. In a separate step, the direct-sun measurements can be combined with the concomitant radiance measurements to derive the aerosol single scattering albedo, asymmetry factor, aerosol phase function, and other parameters using inversion algorithms (see, e.g., Dubovik 2006). These inversions often make use of sky radiance measurements. 


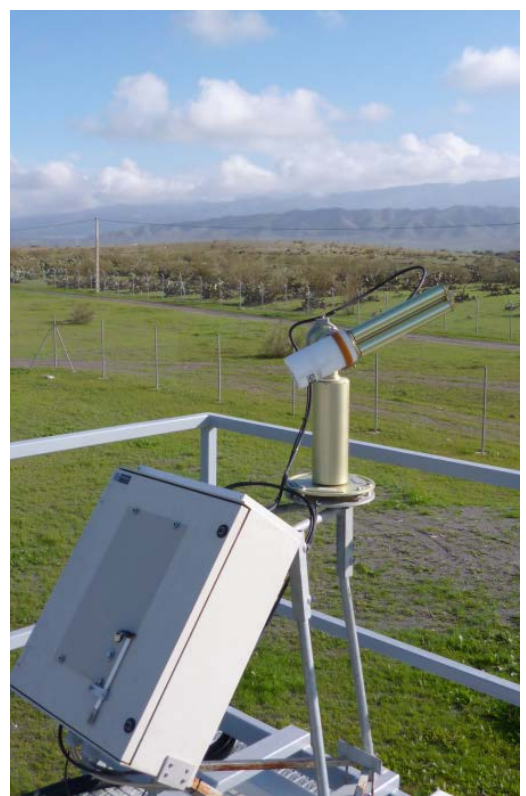

Figure 3-21. AERONET sun photometric station at the Plataforma Solar de Almería. Photo from $D L R$

It is appropriate to mention the two main sun photometer networks in the world: AERONET (http://aeronet.gsfc.nasa.gov/new web/index.html) and SKYNET (http://atmos2.cr.chibau.jp/skynet/). These networks are important for solar resource assessment due to the large number of available observing stations and the applied quality assurance and calibration methods.

The proper determination of aerosol properties can only be done if the solar disk is not covered by clouds. Therefore, cloud filters are used to post-process the data. With AERONET, for example, the spectral DNI measurements are taken in so-called direct-sun triplets. In such a triplet, three series of measurements are made directly after each other. In each series, all different filters are used. By comparing the total optical depth derived from the spectral data of the three series to each other and to defined limits, cloud episodes can be detected (see Smirnov et al. 2000). This cloud screening relies on the higher temporal variability and higher value of cloud optical depth compared to AOD. Data from measurement networks are available in different levels of quality control. Besides cloud screening, the quality control procedures involve various other criteria. In the case of AERONET, for instance, the best data quality ("Level 2") includes manual outlier rejection and correction for the change of the calibration constants before and after a measurement period of one year (Holben et al. 2006). The instrument's calibration is obviously of central importance for the overall data accuracy and thus is performed by each network in a specific way. Whenever available, the highest data quality level should be used.

\subsection{Measurement Station Design Considerations}

To collect useful solar resource data, the successful design and implementation of a solar resource measurement station or network of stations requires careful consideration of the elements summarized in this subsection. 


\subsubsection{Location}

The primary purpose of setting up a solar resource measurement station is to collect data that allow an analyst to accurately characterize the solar irradiance and relevant meteorological parameters at a particular location. Ideally, the instruments would be collocated with the targeted analysis area. In some cases, however, separation distances may be tolerated depending on the complexities of local climate and terrain variations. Lower variability in terrain and climate generally translates to lower variability in the solar resource over larger spatial scales. These effects should be well understood before determining the final location of a measurement station. Refer to Chapters 2 and 8 for more discussion of the effect of distance between station and plant site. The proximity to the target area must be weighed against operational factors, such as availability of power, communications, and access for maintenance, as discussed below. Considerations should also include the possible effects of local sources of pollution or dust-for example, traffic on a nearby dirt road that could impact the measurements.

When measurement stations are constructed in metropolitan areas, industrial areas, or even electrical substations, consideration should be given to possible sources of radio frequency signals that could impart unwanted noise in sensors or cables. For example, the same high building that would provide an attractive unobstructed site for solar measurements may also be the ideal location for radio or television broadcast towers or some other communication apparatus. Such sites should be investigated for interference with the irradiance sensors and monitoring station.

Instrument selection is a fundamental consideration because measurements with greater accuracy will better reflect the actual resource; however, instrument placement is also an important consideration. If nearby objects - such as trees or buildings - shade the instruments for some period of time during the day, the resulting measurement will not truly represent the available solar resource. Distant objects - especially mountains - may be legitimate obstructions, as the shadows they cast are likely to produce an influence beyond the area local to the instruments. Conversely, nearby objects can potentially reflect solar radiation onto the instruments, hence resulting in measurements that do not represent the local natural environment. Such cases may include a nearby wall, window, or other highly reflective object. The best practice is to locate instruments away from any objects that are in view of the instrument detector. The recommendations from WMO (2010) for radiation and all other measurands apply.

The easiest way to determine the quality of solar access is to scan the horizon for a full $360^{\circ}$ of azimuth and note the elevation of any objects protruding into the sky above the local horizon. Look for buildings, trees, antennae, power poles, and even power lines. Most locations will have some obstructions but whether they will be significant in the context of the necessary measurements must be determined. Generally, pyranometers are very insensitive to sky blockage within approximately $5^{\circ}$ elevation above the horizon. Pyrheliometers, however, are more sensitive because objects can completely block DNI, depending on the daily path of the sun throughout the year. The duration and amount of daily blockage are related to the object's width and height above the horizon. On an annual basis, the number of blockage days depends on where along the horizon the object lies. To be a concern, the object must be in the area of the sun near sunrise or sunset, the time and azimuth of which vary throughout the year. For most of the horizon, objects blocking the sky will not be a factor because the sun rises in a limited angular range in the east and sets likewise in the west during sunset (e.g., at $40^{\circ} \mathrm{N}$ latitude, sunrise 
occurs at approximately $60^{\circ}$ from true north at the summer solstice and $120^{\circ}$ from true north at the winter solstice). However, the farther north in latitude the site is located, the greater the angular range of these sunrise and sunset areas of interest. A solar horizon map, or even a sketch of obstructions by elevation and azimuth, will help determine the areas where horizon objects will affect the measurement (see Figure 2-5).

Considerations for locating a station should also include environmental concerns, such as wildlife habitat, migratory paths, drainage, and antiquities or archeological areas.

\subsubsection{Station Security and Accessibility}

Measurement stations can cost tens of thousands or even hundreds of thousands of dollars. Although this equipment is typically not the target of thieves seeking property for resale, it is still subject to theft and should be protected. Vandalism may be even more likely than theft. The less visible and accessible the station is to the public, the less likely it will be the target of theft or vandalism. For example, instruments mounted on a rooftop are less likely to attract unwanted attention than those unprotected beside a highway. Lack of visibility is the best defense against vandalism.

Security fences should be used if people or animals are likely to intrude. Fencing should be at least 1.8-m tall, preferably with barbed wire and fitted with locking gates in high-profile areas where intrusion attempts are unlikely. Less elaborate fences may suffice in areas that are generally secure and where only the curious need be discouraged from meddling with the equipment. In remote venues with few human hazards, cattle fence paneling (approximately 1.2$\mathrm{m}$ tall) may be advisable if large animals roam the area. The fencing should be sturdy enough to withstand the weight of a large animal that may rub against the compound or otherwise be pushed or fall against the fence. It may not be possible to keep smaller animals out of the station compound, and precautions should be taken to ensure that the equipment, cabling, and supports can withstand encounters with these animals. Rodents, birds, and other wildlife may be able to move through the wires or jump over or burrow under fences. In particular, signal cabling between modules or sensors at or near ground level is prone to gnawing by rodents and should be run through a protective conduit or buried. Any buried cable should either be specified for use underground or run through conduit approved for underground use. Underground utilities and other objects should be investigated before postholes are dug or anchors sunk.

If fences are used, they must be considered as a potential obstacle that can shade the instruments or reflect radiation to the instruments. The radiometers should be positioned at least above the line between the horizon and the fence (including barbed wire), if only by a few millimeters, to prevent any shading of the sensor. This assumes that the pyranometer is mounted in a horizontal position and that the pyrheliometer is installed in a solar tracker. Tilted pyranometers should have an unobstructed view of the ground and sky in front of them. In any case, the recommendations from WMO (2014) concerning obstacles have to be followed. If nearby towers are unavoidable, the station should be positioned between the tower and the equator (e.g., to the south of the tower in the northern hemisphere) to minimize shading. The radiometers should be positioned as far as possible from the tower - at least several meters - so the tower blocks as little of the sky as possible (radiometer signal cables should be shorter than $50 \mathrm{~m}$ to avoid losses caused by line resistance). The tower should also be painted a neutral gray to minimize strong reflections that could contaminate the solar measurement. These guidelines assume that the 
tower is part of the measurement station proper and that the site operator has control of the placement or modification of the tower. Without that control, the radiometers should be moved as far as possible from the tower.

Access to the equipment must also be part of a station's construction plan. Because routine maintenance is a primary factor affecting data quality, provisions must be made for reasonable and easy access to the instruments. Factors here could include ease of access to cross-locked property, well-maintained all-weather roads, and roof access that might be controlled by other departments. Safety must also be a consideration. Locations that present hazardous conditionssuch as rooftops without railings or that require access using unanchored ladders — must be avoided.

\subsubsection{Power Requirements}

Ongoing measurements require a reliable source of electrical power to minimize system downtime from power outages. In some areas, power from the utility grid is reliable, and downtime is measured in minutes per year. In other areas, multiple daily power interruptions are routine. Depending on the tolerance of the required analysis to missing data, precautions should be taken to ensure that gaps in the data stream from power outages do not seriously affect the results. The most common and cost-effective bridge for power outages is an uninterruptible power supply (UPS). The UPS can also filter out unwanted or harmful line voltage fluctuations that can occur for a variety of reasons. It has internal storage batteries that are used as a source of power in the event of an AC power interruption. When the AC power is interrupted, internal circuitry makes an almost seamless switch from grid-connected AC power to AC provided through an inverter connected to the battery bank. When power is restored, the UPS recharges the internal battery from the AC line power. Power loss is detected quickly, as is switching to battery, and it is measured in milliseconds or partial line cycles. Some equipment may be particularly susceptible to even millisecond power interruptions during switching and should be identified through trial and error to avert unexpected downtime despite use of the UPS.

The UPS is sized according to:

- Operating power: How much can it continuously supply either on or off grid-connected AC power?

- Operating capacity: How long can the UPS supply the power if the grid connection is interrupted?

Users should estimate the longest occurring power outage and size the UPS for the maximum load of attached devices and the maximum period of battery capacity. Batteries should be tested regularly to ensure that the device can still operate per design specifications. This is most important in hot areas (such as deserts) because batteries may overheat and become inoperative (temporarily or permanently). Internal battery test functions sometimes report errors only when batteries are near complete failure and not when performance has degraded. A timed full-poweroff test should be conducted periodically to ensure that the UPS will provide backup power for the time needed to prevent measurement system failure.

In remote locations where utility power is not available, local power generation with battery storage should be devised. Options for onsite electrical power generation include PV or small 
wind turbine systems (or both) and also gasoline- or diesel-fueled generators. The renewable energy systems should be sized to provide enough energy for the maximum continuous load and power through several days of adverse conditions (cloudy weather and/or low wind speeds). This would include sites prone to persistent surface fog. The sizing is a function of the extremes of the solar climate and should consider the longest gap during reduced generation, the shortest recharge period available after discharge, and the generation capacity and storage necessary to provide uninterrupted power for the target location. Some oversizing is necessary to accommodate degradation of PV panels and battery storage, and consideration should be given to ambient temperature, which affects the ability of a battery to deliver energy. Sizing calculators are available to help with this effort. ${ }^{2}$

Equipment should be specified and tested for self-power-on capability in the event of a power outage. This ensures that when power is restored, the equipment will automatically resume measurements and logging without operator intervention. This is an important consideration for remote locations where considerable downtime might occur before personnel could be dispatched to restart a system.

\subsubsection{Grounding and Shielding}

Station equipment should be protected against lightning strikes and shielded from radio frequency interference that could damage equipment or reduce the validity of the measurements. Several books are available that describe techniques for grounding and shielding low-voltage signal cables (see, e.g., Morrison 1998). Those designing solar resource measurement systems are urged to consult available references and seek expert technical advice.

In general, the following steps should be taken when designing and constructing a measurement station:

1. Use a single-point ground (e.g., a copper rod driven several feet into the ground) for all signal ground connections to prevent ground loops that can introduce noise or biases in the measurements.

2. Use twisted pair, shielded cables for low-voltage measurements connected as doubleended measurements at the data logger. Double-ended measurements require separate logger channels for + and - signal input conductors. These inputs are compared to each other, and therefore, the possibilities for electrical noise introduced in the signal cable are significantly reduced.

3. Physically isolate low-voltage sensor cables from nearby sources of electrical noise, such as power cables. Do not run signal cables in the same bundle or conduit as $\mathrm{AC}$ power cables. If a power cable must cross a signal cable, always position the two at right angles to each other. This limited contact will minimize the possibility of induced voltages in the signal cable. Also, although this case is not recommended, the data logger settings should be selected to avoid signal noise (integration time of the voltage measurement adjusted to $\mathrm{AC}$ frequency; see Section 3.4.5).

\footnotetext{
${ }^{2}$ See http://pvwatts.nrel.gov/.
} 
4. Connect metal structures such as masts and tripods to the ground to provide an easy path to the ground in the event of a lightning strike. This will help protect sensitive instruments. Electronic equipment often has a special ground lug and associated internal protection to help protect against stray voltages from lighting strikes. These should be connected with a heavy gauge wire to ground (12 American wire gauge or larger). Metal oxide varistors, avalanche diodes, or gas tubes can be used to protect signal cables from electrical surges such as lightning. These devices must be replaced periodically to maintain effectiveness. The replacement frequency is a function of the accumulated energy dissipated by the unit.

\subsubsection{Data Loggers}

Most radiometers output a voltage, current, or resistance that is measured by the data logger comprising a voltmeter, ammeter, and/or ohmmeter. The measured output value is subsequently converted to the units of the measurand through a multiplier and/or an offset determined by calibration to a recognized measurement standard.

Data loggers should be chosen so that the measurement uncertainty of the logger is consistent with the uncertainty of the sensor-for example, a much smaller uncertainty, perhaps 3-10 times smaller than the estimated measurement uncertainty associated with the radiometer. This is the accuracy ratio between the data logger and the radiometer. For example, typical specifications for a good data logger measuring a $10-\mathrm{mV}$ output from the radiometer accurate to $1 \%$, or $0.1 \mathrm{mV}$ $(100 \mu \mathrm{V})$, are on the order of total uncertainty (accuracy) of better than (less than) $0.1 \%$ of reading (or full scale) for the parameter in question, which would be $0.010 \mathrm{mV}$, or $10 \mu \mathrm{V}$.

The logger should also have a measurement range that can cover the signal at near full scale to best capture the resolution of the data. For example, a sensor with a full-scale output of $10 \mathrm{mV}$ should be connected to a logger with a range that is at least but not below $10 \mathrm{mV}$. A logger with a $1-\mathrm{V}$ range may be able to measure $10 \mathrm{mV}$ but not with the desired accuracy and resolution. Most modern data loggers have several range selections, allowing the user to optimize the match for each instrument. Because of the nature of solar radiation, radiometers (e.g., pyranometers used for GHI measurements) can sometimes produce $200 \%$ or more of clear-sky readings under certain passing cloud enhancement conditions, and the logger range should be set to prevent over-ranging during these sky conditions. The absolute GHI limit that can be reached during cloud enhancement situations is a decreasing function of the measurement time step. At 1-minute resolution, a safe limit seems to be $1,800 \mathrm{~W} / \mathrm{m}^{2}$. It could reach $2,000 \mathrm{~W} / \mathrm{m}^{2}$ or more at 1 -second resolution with photodiode radiometers. See Gueymard (2017a, 2017b) for more details.

Some radiometers use amplifiers to raise the instrument output to a higher range to better satisfy signal range matching requirements; however, such amplifiers will add some uncertainty to the data with nonlinearity, noise, temperature dependence, or instability. High-quality amplifiers may minimize these effects and allow a reasonable trade-off between logger cost and data accuracy. Calibrations must be made of these radiometer systems by including the pyranometer or pyrheliometer and its uniquely associated amplifier.

The logging equipment should also have environmental specifications that are compatible with the environment where the equipment will be used. Loggers used inside an environmentally controlled building could have less stringent environmental performance specifications than one 
mounted outside in a desert or arctic environment. Equipment enclosures can create an internal environment several degrees above ambient air temperature because of solar heating (absorption by the enclosure materials), heat generated by electronic devices mounted inside, and lack of sufficient ventilation to help purge heat.

The sampling rate and resolution of the solar resource data should be determined from the desired data analysis requirements. The sampling rate refers to how often the logger measures in a time interval. The time resolution is the length of the time interval, which is represented by one data point in the logger's output file. Monthly means, daily totals, hourly, minute, or sub-1minute data records can be of interest. Data loggers can generally be configured to produce output of instantaneous or integrated values at any reasonable time period consistent with the radiometer time-response characteristics. The design should consider the current requirements and, if convenient and practical, future needs for additional analyses. A high-temporal-resolution data-logging scheme can be down sampled or integrated to longer time periods but not the other way around. For example, transforming hourly data to 1-minute data with any certainty and accuracy is impossible if a specific data time series must be reproduced. Data logging equipment, data transfer mechanisms, and data storage can generally handle 1-minute data resolution, and this time realm should be considered the fundamental resolution in the data logger. A resolution of 1 minute or better is recommended to allow for accurate data quality control. Because most applications address the solar energy available over time, integrating data of sub-minute samples within the data logger is a common method of data output regardless of the final data resolution required by the analysis. For instance, 1-second signal sampling is recommended for irradiance measurements in the Baseline Surface Radiation Network (BSRN; McArthur 2005) so that 60 samples are averaged to the reported 1-minute data. The output of instantaneous samples is much less likely to represent the available energy and should be avoided when configuring a data logger. If the size of a measured data set is a defining issue (e.g., limited data communications throughput), the user can determine the lowest temporal resolution necessary for the application and optimize the data collection accordingly.

\subsubsection{Data Communications}

Provisions should be made for automatically and frequently transferring data from the data logger to a data processing facility. This is the basis for adequately frequent data control and timely corrections of outages and errors. Such frequent connections also allow automatic data logger clock corrections for the case that a preferably used local GPS device is not available. Noticeable clock corrections of more than 1 second should never be necessary. Historically, data have been captured, transferred, and processed in various ways. Today, electronics and telecommunications allow remote data collection from nearly any location. One option uses a physical connection between logger and a computer that is used for further data analysis or that forwards the data via an Internet connection. To avoid a cable connection, a cell phone network can be configured to provide virtual Internet links between a measurement station and the data center. Satellite up- and down-links are also available for data transfers in areas that are not served by either wire- or cell-based phone service. Within the area of an observing station, wireless communications such as Wi-Fi connectivity may be useful to minimize the need for long cables between radiometers and data loggers. Depending on the antennas, data can be transferred over distances of a few kilometers. Such distances can occur between the data logger and the control room in big solar power plants with several megawatts of electrical design power. 
To prevent data loss in case of connection problems, the memory of the data logger should be selected appropriately. Memory extensions are available for many data loggers with external cards.

\subsubsection{Operations and Maintenance}

Proper O\&M practices are essential for acquiring accurate solar resource measurements. Several elements in a chain form a quality system. Collectively, these elements produce accurate and reliable solar resource data: station location, measurement system design, equipment installation, data acquisition, and O\&M practices. Proper O\&M requires long-term consistency, attention to detail, and a thorough appreciation for the importance of preventative and corrective maintenance of sensitive equipment.

Calibrations are performed with clean instrument optics and a carefully aligned/leveled instrument. To properly apply the calibration factor, the instrument should be kept in the same condition during field measurements as during the calibration. To maintain the calibration relationship between irradiance and radiometer output, proper cleaning and other routine maintenance is necessary. All O\&M should be carefully documented with log sheets or preferably with electronic databases that contain enough information to reveal problems and solutions or that assert that the instruments were in good form when inspected. The exact times of the maintenance events should be noted rather than estimated. Time-stamped pictures taken before and after maintenance with a camera can be extremely useful to evaluate the importance of soiling and misalignment, for example. A button connected to the data logger that is pressed at the beginning and at the end of an inspection is also recommended. The O\&M information enables an analyst to identify potentially bad data and also provides important documentation to determine and defend the overall quality of the measurements.

The maintenance process includes:

- Checking the alignment/leveling of the detector. Pyrheliometers must be accurately aligned with the solar disk for accurate DNI measurements. Pyranometer detectors must be horizontal for GHI and DHI measurements and accurately tilted (or aligned with a flat-plate collector) for GTI measurements. The radiometer orientation should be checked periodically using the features described earlier in this chapter.

- Cleaning the instrument optics. To properly measure the solar irradiance, no contaminant should block or reduce the radiation falling on the detector. The outdoor environment provides many sources of such contamination, such as dust, precipitation, dew, plant matter, insects, and bird droppings. The sensors should be cleaned regularly to minimize the effect of contaminants on the measurements. In many cases, this can require daily maintenance of radiometers.

- Documenting the condition of the radiometer. For analysts to understand limitations of the data, conditions that affect the measurements must be documented. This includes substandard measurement conditions, but it is just as important to document proper operations to add credibility to the data set. Observations and notes provide a critical record of conditions that positively and negatively affect data quality. 
- Documenting the environment. As a consistency check, note the sky and weather conditions at the time of maintenance when interpreting data from the radiometer, including measurements with unusual values.

- Documenting the infrastructure. The measurement station as a whole should be examined for general robustness. Any defects should be noted and corrected.

Maintenance frequency depends on prevailing conditions that soil the instruments. This includes dust, rain, dew, snow, birds, and insects. It also depends on instrument type. Radiometer designs based on optical diffusers as the surface separating the inside of the instrument from the environment are less susceptible to dust contamination than instruments with clear optics, such as domed pyranometers (Myers et al. 2002). This is caused by the fact that fine soiling particles scatter much more than they absorb solar radiation. Absorption affects instruments with clear optics and diffusers the same way. In contrast, the scattering-induced soiling effect has less impact on instruments with diffusers because the latter can transmit most of what particles just scattered. The scattered radiation (mostly in the forward direction) hence reaches the detector in nearly the same way as radiation would enter a clean diffuser. Conversely, the scattering often causes the incoming radiation to miss the detector in instruments with clear optics because the latter is at some distance from the former. This is especially relevant for pyrheliometers (Geuder 2006). Soiling of windowed or domed radiometers can quickly affect their reading and increase their measurement uncertainty. This explains why thermopile radiometers must be cleaned very frequently (e.g., on a daily basis). As described earlier, using a ventilator for a pyranometer can reduce this risk of contamination; thus, the frequency and cost of maintenance should be important considerations to take into account for proper instrument specification. Although sensors with diffusers, such as RSIs, are not prone to strong soiling effects, they still require regular cleaning (e.g., twice a month). Note that a diffuser below a clear entrance window/dome does not have an advantage compared to a thermopile below the same clear entrance window/dome.

The examples above mentioning daily cleaning for sensors with clear optics and cleaning twice a month for sensors with diffusers as outer surface are useful for many sites. However, the cleaning interval should be defined for each site at the beginning of the measurement period by analyzing the immediate effect of cleaning on the measurement signal. Depending on the noted period after which soiling significantly influences the measurement, the cleaning interval should be adjusted so that the degradation in sensitivity never reaches $\approx 1 \%$. Each cleaning period and the state of the sensors should be documented and the measurement values should be checked to evaluate the effect of cleaning on the recorded values.

Radiometers should be carefully cleaned at each inspection, even if soiling appears minimal. Cleaning is generally a very short procedure. A recommendation for the cleaning procedure is as follows. First, remove any loose particles from the entrance window with a soft brush or compressed air. Then clean the entrance window, dome, or diffuser with a dry cloth. If dirt remains after this step, wet a second cloth with distilled water (or methyl hydrate) and wipe the window/diffusor/dome clean. If ice sticks on the surface, try melting the ice with one's hands or a hair dryer. More aggressive methods might damage the entrance windows and are therefore not recommended. 
Collimators without entrance windows (as used in active cavity radiometers) noticeably reduce the accumulation of dust on the sensor's entrance optics, but they may still be affected by insects or spiders because they can enter the collimators, causing strong signal reductions. Even a single fiber of a spider web can significantly reduce the signal. Such collimators must therefore be inspected frequently.

At remote sites that may be too difficult to maintain during extended periods, a higher-class windowed instrument might not be optimal, despite its potential for better measurements. The cost of maintenance for a remote site may dominate the estimated cost of setting up and operating a station. This aspect should be anticipated when planning a measurement campaign. Often, less maintenance-intensive sensors with initially lower accuracy compared to windowed instruments can be a better choice, at least until the station becomes permanently serviceable on a daily basis.

Additional spot inspections should be conducted after significant weather events (e.g., dust storms, snow storms, heavy rainfall, rainfall during periods with high optical depth, and storms). Radiometer optics may not necessarily soil within a 24-hour period, but the effects of soiling can best be mitigated with frequent inspection.

Maintenance at remote measurement sites away from institutional or corporate employment centers will require finding a qualified person nearby who can perform the necessary maintenance duties. The qualifications for maintenance are generally nontechnical, but they require someone with the interest and disposition to reliably complete the tasks. As a rule, compensating these people for time and vehicle mileage - rather than seeking volunteersbecomes a worthwhile investment in the long run because it sets up a firm contractual commitment to perform all necessary maintenance duties. Without that formal relationship, it can become difficult to assert the need for reliable and regular attention.

A general conclusion is that a conservative maintenance schedule will support the credibility of the measurement data set and provide the analyst with a base of justification when assigning confidence intervals for the data.

\subsection{Data Quality Control, Data Correction, Data Quality Assessment, and Metadata}

The data quality is in great parts established when the measurement is taken. Little can be done if errors occurred during the measurement to improve fundamental quality. For example, a poorly maintained station with dirty optics or misaligned instruments will produce data with presumed (or even apparent) errors, and the magnitude of those errors is not likely to be discernable until days or weeks later. Often one can only guess at which a posteriori corrections to make. In this context, data quality control involves a well-defined supervisory process by which station operators are confident that, when a measurement is taken with unattended instruments, the instruments are in a state that produces data of known quality. This process largely encompasses the calibration, inspection, and maintenance procedures discussed earlier, along with log sheets and other items that document the condition of the station. It also includes a critical inspection or assessment of the data to help detect problems not evident from physical inspection of the instruments. 
Data quality assessment is a method by which data quality can be judged based on criteria for a particular application. Several particular errors of meteorological data can be detected by automatic screening algorithms. Corresponding tests for radiation data are documented in a number of publications, including Long and Dutton (2002); Maxwell et al. (1993); Wilcox and Cormack (2011); Journée and Bertrand (2011); Espinar et al. (2011); and Perez-Astudillo et al. (2016). Auxiliary data of direct interest can also be tested as explained in Geuder et al. (2015). The main parameters discussed there are: logger and battery voltage, logger temperature, speed of ventilation units, and meteorological measurands such as wind speed and pressure. Data can be compared to certain physical limits that have been determined to be reasonable, with redundant or complementary measurements, or with physical or empirical models - all of which will provide some degree of independent measure for a quality judgment. The temporal behavior of data can be used to identify errors, such as blocked wind vanes or damaged cables (Geuder et al. 2015). However, dependent on how strict the screening parameters and their corresponding values are chosen; too many or too few events may be detected. Moreover, the values of some parameters are dependent on the local characteristics of the site and its weather conditions. Therefore, the results of the automatic screening always demand a manual check by an expert to ensure their validity. Finally, additional data issues potentially known by the station's supervisor have to be included as comments or flags.

The interpretation and application of solar resource measurements depend greatly on the efforts to record and include metadata relevant to the observations. This includes site location; local horizon survey; data acquisition system(s); input signal channel assignments; and radiometer types, models, serial numbers, calibration histories, and installation schemes, as well as information on eventual post processing of the data and maintenance records. An example of online metadata is available from NREL's Solar Radiation Research Laboratory. ${ }^{3}$ Such metadata should be included with the archiving of the measured solar resource data. For instance, issues that have to be documented may include damaged or misaligned sensors, maintenance works on the instruments, detection of soiled sensors and subsequent sensor cleaning, obstructed sensors, and temporarily erroneous calibration constants in the program code. These events are frequently not detected automatically or sometimes not even detectable by automatic quality-control screening tools. Hence, manual checks are required. However, automatic tests such as those presented in what follows contain important and valuable information and, thus, should always be performed.

One common method for evaluating DNI, GHI, and DHI quality is a three-component closure test. As described in Chapter 2, the measurements of DNI and DHI can be combined mathematically to derive GHI, as described in Equation 2-2. When all three components are measured, measurement redundancy is apparent because any one component can be derived from the other two. Hence, in the context of quality assurance, the expected values of each component can be calculated from any other two. This method helps quantify the relative error among the three components, although it does not automatically determine strictly which specific measurement — or measurements - are in error. However, operational knowledge of the instruments and trackers can provide valuable insight into likely errors. A frequent problem is

\footnotetext{
${ }^{3}$ See http://www.nrel.gov/midc/srrl bms.
} 
caused by malfunction of the tracker. If the pyrheliometer does not point at the sun, DNI is $\approx 0$ and $\mathrm{DHI} \approx \mathrm{GHI}$. This situation may last for hours and can be incorrectly interpreted as the signature of an overcast sky. Similarly, a slightly misaligned tracker would cause a too low DNI and too high DHI. With this information, combined with the visual detection of trends in the magnitude of flagging, a data quality expert can quickly spot common operational errors. The independent measurement of the three redundant components - rather than only a single measurement or two components of specific interest-is a significant and important tool for data quality analysis, which should be strongly considered when specifying instrumentation for a station. (Note that even though RSIs provide three components, they actually measure only two of them per se-hence preventing them access to this procedure.)

The addition of data quality flags to the data files is an extremely important step in the whole quality assurance process. For example, the SERI QC software for irradiance measurements (Maxwell et al. 1993) produces flags that can be plotted (see Figure 3-22). The process generates a data quality value, or flag, for each data point based upon a normalization process involving the dimensionless parameters - clearness index (Kt), effective diffuse horizontal transmittance (Kd), and direct beam transmittance $(\mathrm{Kn})$ - derived from the corresponding extraterrestrial radiation (ETR) and SZA in the case of Kt and $\mathrm{Kd}$. As previously mentioned in Chapter 2, $\mathrm{Kt}$ is the ratio between GHI and the corresponding extraterrestrial irradiance on a horizontal plane. Similarly, $\mathrm{Kd}$ is the ratio between DHI and the extraterrestrial irradiance on a horizontal plane, and $\mathrm{Kn}$ is the ratio between DNI and the extraterrestrial normal irradiance. In Figure 3-22, the plot on the left indicates more severe flags from among the three components, plotted here by day of month (y-axis) and hour of day (x-axis). The lowest error is in dark blue, and the largest error in red. Further, the other three plots correspond respectively to normalized GHI, DNI, and DHI measurements, providing the analyst with additional information to pinpoint the measurement causing the error. 


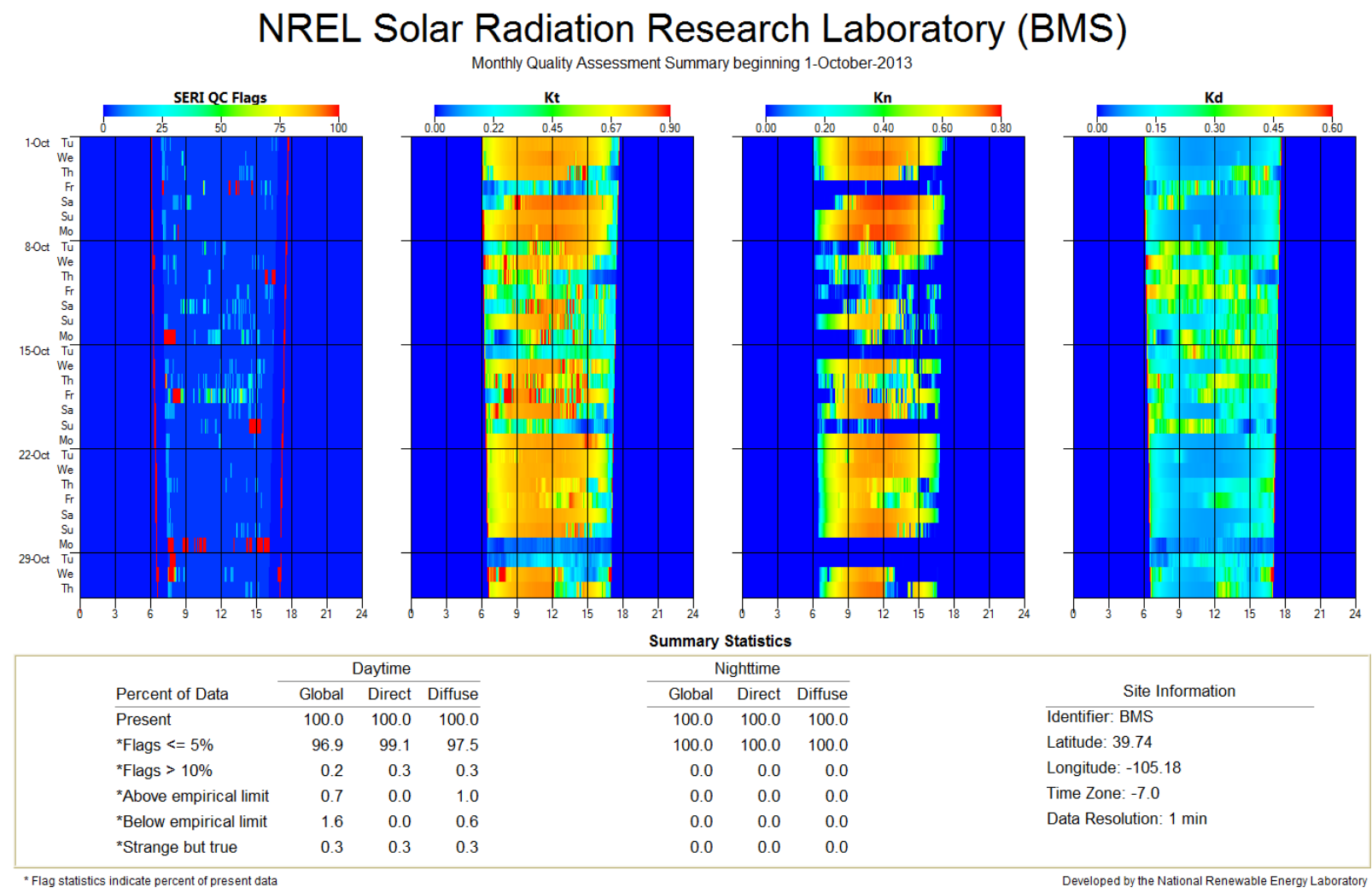

Figure 3-22. Example of SERI quality-control data quality-assurance reporting using SERI-QC software. Image by NREL

In the case shown in Figure 3-22, the three-component data (GHI, DNI, and DHI) were submitted to the SERI QC software, which performs the three-component closure test in the realm of normalized indices (i.e., $\mathrm{Kt}, \mathrm{Kn}$, and $\mathrm{Kd}$ ). This analysis is performed in $\mathrm{K}$-space to remove the SZA effect. Thus, in K-space,

$$
\mathrm{Kt}=\mathrm{Kn}+\mathrm{Kd}(3-5)
$$

Or, rearranged, the deviation from this equation of component closure can be quantified as the residual from

$$
\mathrm{Kt}-\mathrm{Kn}-\mathrm{Kd}(3-6)
$$

Perfect component closure would result in $\mathrm{Kt}-\mathrm{Kn}-\mathrm{Kd}=0$. Hence, any nonzero value indicates some disagreement among the instruments; however, this method does not reveal which component or components are in error-only that there is some disagreement. Further, compensations of errors can result in a "false" zero value. This is the case when, for instance, the tracker is completely misaligned. Other tests are thus necessary to automatically detect this type of error (Long and Shi 2008).

Despite the difficulties described above, a knowledgeable analyst can confidently detect measurement concerns in most typical measurement scenarios. In the case of Figure 3-22, for instance, the residual is displayed as a color code in the leftmost column for each minute data 
record containing the three components. The actual $\mathrm{Kt}, \mathrm{Kn}$, and $\mathrm{Kd}$ values are also plotted in the next three columns; the red and blue colors roughly correlate to high (clear) and low (cloudy) irradiance, respectively.

If only two components are measured (such as with RSI instruments), the powerful threecomponent closure test cannot be used. However, less informative two-component tests can still be applied. These tests make use of the fact that some combinations of GHI and DNI are unlikely (e.g., very high GHI concomitant with very low DNI). To some extent, this limitation can be mitigated by including a secondary unshaded (GHI) pyranometer on the RSI to provide some redundancy. This relatively low-cost method adds confidence in the measurements and can be included in a two- or three-component quality assessment test.

The three-component method described above is generally more reliable than a simple clear-sky data analysis in which some conclusions are drawn based on modeled or other expected values of clear-sky data. Significant day-to-day variations in clear-sky data can occur because of variations in atmospheric constituents, such as aerosols or water vapor. Thus, such natural variations can make it difficult to draw conclusions about possible instrument error without specific information regarding other critical atmospheric components.

A successful quality-control process requires elements of quality assessment and feedback. Figure 3-23 depicts a quality-assurance cycle that couples data acquisition with quality assessment and feedback.

\section{The Quality Assurance Cycle}

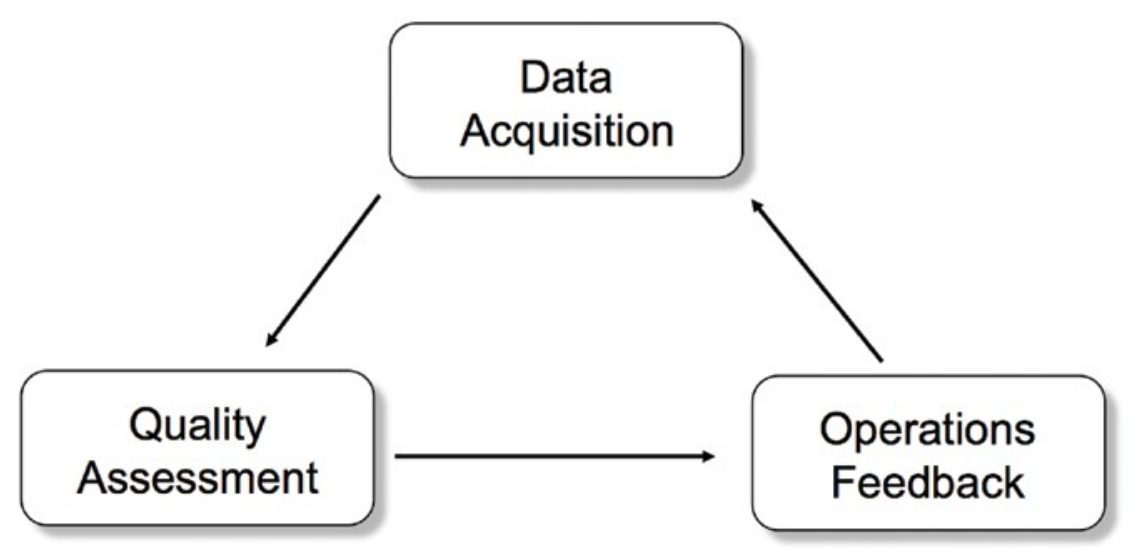

Figure 3-23. Information flow of a quality-assurance cycle. Image by NREL

As shown in Figure 3-23, the information flows from data acquisition to quality assessment, where some criteria are used to establish data quality. The results of the quality assessment are analyzed and formed into feedback that goes back to the data acquisition module. The activities in the boxes can take several forms. For example, quality assessment could be the daily site inspection, and the analysis and feedback could be a simple procedure that corrects equipment malfunctions. Or the quality assessment could be a daily summary of data flags, and the analysis 
then provides a determination of specific instrument error that is transmitted back to maintenance personnel, instructing them to correct deficiencies or further troubleshoot problems.

The faster the cycle runs, the sooner errors will be detected, and the fewer bad data will be collected during failure modes. Conversely, if the site is inspected infrequently, the chances increase that a large portion of the data set would be contaminated with substandard measurements. More than one quality-assurance cycle can — and likely will — run at any time, each with a different period and emphasis, as noted above: daily inspection, weekly quality reports, and monthly summaries.

One practical aspect of this cycle is the importance of positive feedback - a regular report back to site personnel of high-quality operations. This positively reinforces a job well done and keeps site operators cognizant that data are being used and checked and that their efforts are an integral part of an ongoing process.

The quality-assurance cycle is important, and thus should be well defined and funded to maintain consistent data quality over time. After the quality of the data is determined, corresponding conclusions have to be made for the further use of the data. In every case, the quality-assurance data have to be included in the data set as metadata. In some cases, the quality of the data can even be improved based on the quality assurance. For example, data gaps from one sensor can be filled with the redundant data. Gap filling is a complex topic that is not described in detail here. To calculate daily, monthly, or yearly sums, gap filling will nearly always be necessary, and it is recommended that the reader considers various publications concerning the topic for this type of correction (Hoyer-Klick et al. 2009; Espinar et al. 2011; Roesch et al. 2011a; Roesch et al. $2011 \mathrm{~b}$ Schwandt et al. 2014). Because data gaps can rarely be completely avoided in long time series, and gap filling may not always work over long periods of missing data, a critical problem is then to obtain correct estimates of the long-term (e.g., monthly or annual) averages, which are of utmost importance in solar resource assessments. Practical methods have been developed to overcome this problem with the minimum possible loss of accuracy, as described by Roesch et al. (2011a and 2011b).

Another systematic error that savvy analysts may be able to correct concerns the instrument's calibration. If the recalibration of a sensor shows a noticeable change relative to the calibration factor that was used shortly before the recalibration, the data might be reprocessed with a corrected, time-variable calibration factor. For sun photometers, this kind of post processing is applied to AERONET Level 1.5 data to elevate them to Level 2 (Holben et al. 1998). A distinct change in calibration factor can be assumed to be linear in time, and the data between two calibration periods are then reprocessed with a time series of this linearly corrected calibration factor.

Finally, the systematic effects of soiling on measured irradiance data can be reduced a posteriori-at least to some extent. This requires that any change in irradiance following the sensor cleaning be documented. Examples of data correction methods can be found in Geuder (2006) and Bachour et al. (2016). However, such a correction can result in better data only if the soiling effect is small $(<1 \%)$. The availability of such a rough soiling correction method does not eliminate the requirement stated above that instrument cleaning must be done frequently. 


\section{References}

Augustyn, J.,T. Geer, T. Stoffel, R. Kessler, E. Kern, R. Little and F. Vignola. 2002. "Improving the Accuracy of Low Cost Measurement of Direct Normal Solar Irradiance." Solar 2002 Conference Proceedings; June 15-20, 2002, Reno, Nevada.

Bachour, D., D. Perez-Astudillo, and L. M. Pomares. 2016. Study of Soiling on Pyranometers in Desert Conditions. Proc. Eurosun 2016 Conf., Palma de Mallorca, Spain. International Solar Energy Society.

Badosa, J., J. Wood, P. Blanc, C. N. Long, L. Vuilleumier, D. Demengel, and M. Haeffelin. 2014. "Solar Irradiances Measured Using SPN1 Radiometers: Uncertainties and Clues for Development." Atmos. Meas. Tech. 7(12): 4267-83. doi: 10.5194/amt-7-4267-2014.

Dubovik, O. 2006. “AERONET Inversion Products.” Retrieved March 10, 2016. http://aeronet.gsfc.nasa.gov/new_web/Documents/Inversion_products_V2.pdf.

Geuder, N., F. Wolfertstetter, S. Wilbert, D. Schüler, R. Affolter, B. Kraas, E. Lüpfert, and B. Espinar. 2015. "Screening and Flagging of Solar Irradiation and Ancillary Meteorological Data." Energy Procedia 69(0): 1989-98. doi: http://dx.doi.org/10.1016/j.egypro.2015.03.205.

Gueymard, C. A. 2017a. "Cloud and Albedo Enhancement Impacts on Solar Irradiance Using High Frequency Measurements From Thermopile and Photodiode Radiometers. Part 1: Impacts on Global Horizontal Irradiance.” Solar Energy, 153 (2017), pp. 755-765.

- 2017b. "Cloud and Albedo Enhancement Impacts on Solar Irradiance Using High Frequency Measurements From Thermopile and Photodiode Radiometers. Part 2: Performance of Separation and Transposition Models for Global Tilted Irradiance." Solar Energy, 153 (2017), pp. 766-779.

Gueymard, C. A., and D. R. Myers. 2009. "Evaluation of Conventional and High-Performance Routine Solar Radiation Measurements for Improved Solar Resource, Climatological Trends, and Radiative Modeling." Solar Energy 83: 171-85.

Gueymard, C. A., and J. A. Ruiz-Arias. 2015. "Validation of Direct Normal Irradiance Predictions Under Arid Conditions: A Review of Radiative Models and Their TurbidityDependent Performance." Renewable and Sustainable Energy Reviews 45: 379-96.

Habte, A., M. Sengupta, A. Andreas, S. Wilcox, and T. Stoffel. 2016. "Intercomparison of 51 Radiometers for Determining Global Horizontal and Direct Normal Irradiance Measurements. Solar Energy 133: 372-93. DOI: 10.1016/j.solener.2016.03.065

Holben, B. N., T. F. Eck, I. Slutsker, D. Tanre', J. P. Buis, A. Setzer, E. Vermote, J. A. Reagan, Y. J. Kaufman, T. Nakajima, F. Lavenu, I. Jankowiak, and A. Smirnov. 1998. "AERONET-A Federated Instrument Network and Data Archive for Aerosol Characterization." Remote Sensing of the Environment 66: 1-16. 
Holben, B. N., T. F. Eck, I. Slutsker, A. Smirnov, A. Sinyuk, J. Schafer, D. Giles, and O. Dubovik. 2006. "Aeronet's Version 2.0 Quality Assurance Criteria." Presented at Remote Sensing of the Atmosphere and Clouds, November 28.

Hoyer-Klick, Carsten, Fred Hustig, Marko Schwandt, and Richard Meyer. 2009. "Characteristic Meteorological Years From Ground and Satellite Data." Presented at SolarPACES Conference, at Berlin, Germany.

IEC. 2017. IEC 61724-1:2017 Photovoltaic System Performance - Part 1: Monitoring.

Jessen, W., S. Wilbert, B. Nouri, N. Geuder, and H. Fritz. 2016. "Calibration Methods for Rotating Shadowband Irradiometers and Optimizing the Calibration Duration." Atmospheric Measurement Technology (4): 1601-12. doi: 10.5194/amt-9-1601-2016

LI-COR Biosciences 2005. "LICOR Terrestrial Irradiation Sensors: Instruction Manual." Section 5-2. LI-COR, Inc. Lincoln, Nebraska https://www.licor.com/documents/8yfdtw1rs27w93vemwp6

Löf, G. O. G., J. A. Duffie, and C. O. Smith. 1966. "World Distribution of Solar Radiation." Solar Energy 10(1): 27-37. DOI:10.1016/0038-092X(66)90069-7

Long, C.N., E. G. Dutton. 2002. "Baseline Surface Radiation Network: Global Network Recommended QC Tests-V2.0.” Accessed September 25, 2014: http://epic.awi.de/30083/1/BSRN_recommended QC tests_V2.pdf.

Long, C. N., and Y. Shi. 2008. "An Automated Quality Assessment and Control Algorithm for Surface Radiation Measurements.” The Open Atmospheric Science Journal 2: 23-37.

McArthur, L. J. B. 2005. "Baseline Surface Radiation Network (BSRN) Operations Manual Version 2.1.” WCRP-121 WMO/TD-No. 1274. In World Climate Research Programme.

Micek, L.V. (1981).Direct Beam Insolation Measurement Errors Caused by Pyrheliometer Misalignment. M.S. Thesis, Trinity University, San Antonio, TX.

Michalsky, J., E. Dutton, M. Rubes, D. Nelson, T. Stoffel, M. Wesley, M. Splitt, and J. DeLuisi. 1999. "Optimal Measurement of Surface Shortwave Irradiance Using Current Instrumentation." J. Atmos. Oceanic Technol., 16, 55-69.

Myers, D. 2013. "Solar Radiation: Practical Modeling for Renewable Energy Applications.”Catalog No. K14452. Boca Raton, FL: CRC Press, Taylor \& Francis Group, 199 pp. ISBN 978-1-4665-0294-9

Painter, H. E. 1981. The Performance of a Campbell-Stokes Sunshine Recorder Compared With a Simultaneous Record of Normal Incidence Irradiance.” Meteorology Magazine 110: 102-9.

Perez-Astudillo, D., D. Bachour, L. M. Pomares. 2016. "Improved Quality Control Protocols on Solar Radiation Measurements.” Proc. Eurosun 2016 Conf., Palma de Mallorca, Spain. International Solar Energy Society. 
Roesch, A., Wild, M., Ohmura, A., Dutton, E. G., Long, C. N., and Zhang, T.. 2011a. "Assessment of BSRN radiation records for the computation of monthly means,." Atmos. Meas. Tech., 4, 339-354, https://doi.org/10.5194/amt-4-339-2011, 2011.

Roesch, A., M. Wild, A. Ohmura, E. G. Dutton, C. N. Long, and T. Zhang. 2011b. "Assessment of BSRN Radiation Records for the Computation of Monthly Means." Atmos. Meas. Tech 4: 339-54. Corrigendum, Atmos. Meas. Tech., 4, 973, 2011.

Schwandt, Marko, Kaushal Chhatbar, Richard Meyer, Katharina Fross, Indradip Mitra, Ramadhan Vashistha, Godugunur Giridhar, S. Gomathinayagam, and Ashvini Kumar. 2014. "Development and Test of Gap Filling Procedures for Solar Radiation Data of the Indian SRRA Measurement Network." Energy Procedia 57: 1100-9. doi: http://dx.doi.org/10.1016/j.egypro.2014.10.096.

Smirnov, A., B. N. Holben, T. F. Eck, O. Dubovik, and I. Slutsker. 2000. "Cloud-Screening and Quality Control Algorithms for the AERONET Database." Remote Sensing of Environment 73(3): 337-49. doi: http://dx.doi.org/10.1016/S0034-4257(00)00109-7

Stanhill, G. 1998. "Estimation of Direct Solar Beam Irradiance From Measurements of the Duration of Bright Sunshine.” International Journal of Climatology 18(3): 347-54. DOI: 10.1002/(SICI)1097-0088(19980315)18:3<347::AID-JOC239>3.0.CO;2-O

Vignola, F., J. Michalsky, and T. Stoffel. 2012. "Solar and Infrared Radiation Measurements." Ed. Abbas Ghassemi. CRC Press, Boca Raton, FL. 394 pp. ISBN 978-1-4398-5189-0

Vignola, Frank, Josh Peterson, Stefan Wilbert, Philippe Blanc, Norbert Geuder, and Chris Kern. 2016a. "New Methodology for Adjusting Rotating Shadowband Irradiometers Measurements." Presented at SolarPACES, Abu Dhabi.

Vignola, Frank, Zachary Derocher, Josh Peterson, Laurent Vuilleumier, Christian Félix, Julian Gröbner, and Natalia Kouremeti. 2016b. "Effects of Changing Spectral Radiation Distribution on the Performance of Photodiode Pyranometers." Solar Energy 129: 224-35. doi: http://dx.doi.org/10.1016/j.solener.2016.01.047

Vuilleumier, L., M. Hauser, C. Félix, N. Sommer, D. Ruffieux, and B. Calpini. 2012. "Performance Evaluation of Radiation Sensors for the Solar Energy Sector." TECO-2012. WMO Technical Conference on Meteorological and Environmental Instruments and Methods of Observation, Brussels, Belgium, 16-18 October 2012. https://www.wmo.int/pages/prog/www/IMOP/publications/IOM-109 TECO2012/Session1/P1 25 Vuilleumier performance eval radiation sensors.pdf.

Wilbert, S. 2014. "Determination of Circumsolar Radiation and Its Effect on Concentrating Solar Power." Ph.D. Thesis. Aachen, Germany: Rheinisch-Westfälische Technische Hochschule Aachen University. urn:nbn:de:hbz:82-opus-51715. Accessed November 11, 2014: http://darwin.bth.rwthaachen.de/opus3/volltexte/2014/5171/.

Wilbert, S., N. Geuder, M. Schwandt, B. Kraas, W. Jessen, R. Meyer, and B. Nouri. 2015. "Best Practices for Solar Irradiance Measurements with Rotating Shadowband Irradiometers." Solar 
Resource Assessment and Forecasting, International Energy Agency (IEA), Solar Heating \& Cooling Programme (SHC), Task 46, August 18th, 2015. http://task46.iea-

shc.org/data/sites/1/publications/INSRSI IEA-Task46B1 BestPractices-RSI 150819.pdf

Wilbert, Stefan, Stefan Kleindiek, Bijan Nouri, Norbert Geuder, Aron Habte, Marko Schwandt, and Frank Vignola. 2016. "Uncertainty of Rotating Shadowband Irradiometers and SiPyranometers Including the Spectral Irradiance Error." AIP Conference Proceedings 1734(1): 150009. doi: http://dx.doi.org/10.1063/1.4949241

WMO. 2014. Guide to Meteorological Instruments and Methods of Observation. WMO-No. 8, http://library.wmo.int/opac/index.php?lvl=notice_display\&id=12407\#.WDVYPdXyuM8.

Wood, J. 2015. “SPN1 Best Practices.” http://www.delta-t.co.uk/spn1-resource-centre/. 


\title{
4 Modeling Solar Radiation-Current Practices
}

\author{
Manajit Sengupta, ${ }^{1}$ Jesús Polo, ${ }^{2}$ Christian Gueymard, ${ }^{3}$ and Yu Xie ${ }^{1}$ \\ 1. National Renewable Energy Laboratory \\ 2. Centro de Investigaciones Energéticas, Medioambientales y Tecnológicas (CIEMAT) \\ 3. Solar Consulting Services
}

\subsection{Introduction}

High-quality solar resource assessment accelerates technology deployment by making a positive impact on decision making and reducing uncertainty in investment decisions. Global horizontal irradiance (GHI), global tilted irradiance (GTI), and/or direct normal irradiance (DNI) are the quantities of interest for resource assessment and characterization at a particular location. Surface-based measurements of DNI and GHI can be made only on a relatively sparse network, given the costs of operations and maintenance (O\&M). GTI is rarely measured in radiometric networks or in PV deployments. Nevertheless, observations from ground networks have been used in conjunction with models to create maps of surface solar radiation (Gueymard 2008a). Another option is to use information from geostationary satellites to estimate GHI and DNI at the surface (Cano et al. 1986, Diabate et al., 1989; Beyer et al., 1996; Perez et al., 2002; Rigollier et al., 2004; Cebecauer et al., 2010 ; Qu et al., 2016, Pinker and Laszlo 1992). Because different geostationary satellites are available at different longitudes around the world, radiation can be available for the entire globe (at least between approximately -60-degrees and +60 -degrees latitudes) at temporal and spatial resolutions representative of a particular satellite. For northern and southern latitudes, a compilation of satellite data from polar orbiting offers good spatial but at a lower temporal resolution (e.g., Karlsson et al. 2017).

Solar radiation models that use only ground-measured input parameters were used in the past when satellite or weather-model-derived databases were not available. Examples of such models are briefly mentioned for historic reasons. One popular historic model type is based on data from the Campbell-Stokes sunshine duration recorder. The monthly mean GHI is derived using a regression fit to the number of sunshine hours measured by the sunshine recorder's burn marks when direct solar irradiance exceeds a threshold value of $120 \mathrm{~W} / \mathrm{m}^{2}$. The regression coefficients are calculated using existing GHI measurements at specific locations. The exact method to calculate GHI using sunshine recorder information is empirical and therefore specific to each geographical area. Moreover, the meteorological services of some countries, such as the United States and Canada, have stopped measuring sunshine duration because of the limited quality and significance of this measurement, which is not standardized and varies from country to country.

In the absence of surface radiation measurements, estimates of surface radiation can also be made using meteorological ground measurements such as cloud cover, temperature, visibility, and water vapor in a radiative transfer model (Maxwell 1998; Marion and Wilcox 1994). Methods have been developed over the years and have been used to create earlier versions of the U.S. National Solar Radiation Data Base (NSRDB (1991-2005)), for instance (George et al. 2007). The METeorolgoical-STATistical (METSTAT) model (Maxwell 1998) uses information about cloud cover, water vapor, ozone, and aerosol optical depth (AOD) to develop empirical correlations to compute atmospheric transmittance extinction during both clear- and cloudy-sky 
conditions. Similar developments have been carried out in Europe with the successive versions of the European Solar Radiation Atlas (Page et al. 2001).

Long-term GHI data can also be obtained from various numerical weather prediction (NWP) models, either by operating them in reanalysis mode or from actual operational weather forecasts. Examples of reanalysis data include the ERA interim data from the European Center for Medium-Range Weather Forecasting (ECMWF) and the Modern Era Retrospective analysis for Research and Applications, Version 2 (MERRA-2) from the National Aeronautics and Space Administration (NASA). Weather forecasts such as those from ECMWF's Integrated Forecasting System (IFS) and the National Oceanic and Atmospheric Administration's (NOAA's) Global Forecast System (GFS) can also provide estimates of GHI. Such estimates, however, are typically not as accurate as those derived from satellite-based models and require careful bias corrections (Boilley and Wald 2015).

This chapter contains an introduction to satellite-based models, information about currently operational models that provide surface radiation data for current or recent periods, a summary of radiative transfer models used in the operational models, and a discussion of uncertainty in solar-based resource assessment. A short discussion on NWP-based solar radiation data is also included.

\subsection{Estimating the Direct and Diffuse Components from GHI}

During clear and partly cloudy conditions, diffuse irradiance on a horizontal surface, DHI, is often a relatively small part $(<30 \%)$ of GHI. During dense overcast conditions, GHI and DHI should be identical. When no simultaneous DHI or DNI measurements exist, and no alternate determinations from, for example, physical-based satellite-based models are available, DNI and DHI must be estimated from GHI data. Many models based on empirical correlations between GHI and either DHI or DNI data have been developed since Liu and Jordan (1960), Erbs et al. (1982), Maxwell (1987), Perez et al. (1990), Louche et al. (1991), Ruiz-Arias (2010), and many others have developed algorithms for estimating hourly DHI or DNI from GHI. More recently, Engerer (2015), Gueymard and Ruiz-Arias (2016), and Aler et al. (2017) extended this method to obtain DNI and DHI at 1-minute resolution. These algorithms use empirical correlations between the global clearness index, $\mathrm{Kt}=\mathrm{GHI} /[\mathrm{ETR} \cos (\mathrm{SZA})]$, and the diffuse fraction, $\mathrm{K}=\mathrm{DHI} / \mathrm{GHI}$, the diffuse clearness index, $\mathrm{Kd}=\mathrm{DHI} /[\mathrm{ETR} \cos (\mathrm{SZA})]$, or the direct clearness index, $\mathrm{Kn}=$ DNI/ETR. All these separation models are derived empirically. There are reviews of substantial literature on this topic (e.g., Gueymard 2008a; Gueymard and Ruiz-Arias 2016; Tapakis et al. 2016). Analysts should note that some hourly separation models, including the most popular ones, might not perform correctly if used with sub-hourly data (Gueymard and Ruiz-Arias 2016).

\subsection{Estimating Irradiance on a Tilted Surface}

Solar conversion systems, such as flat-plate collectors or non-concentrating photovoltaics (PV), are tilted toward the equator to increase their solar resource. Estimating or modeling the irradiance incident upon them is essential to predict their performance and yield. This irradiance incident on the plane of array (POA) is usually called GTI, or sometimes simply POA. GTI can be measured directly by pyranometers that are tilted the same as the collector plane. Modeling GTI mainly requires data of the three main components on the horizontal surface (GHI, DNI, and diffuse horizontal irradiance [DHI]). GTI can be estimated as the sum of the incident beam, 
incident sky diffuse, and incident ground-reflected irradiances on the tilted surface. The incident beam contribution is simply a straightforward geometric transformation of DNI, just requiring the angle of incidence of DNI on the tilted plane. The ground-reflected contribution is generally small for tilts below $45^{\circ}$, unless the ground is covered with snow. A simple estimation is possible but requires a number of assumptions: The foreground is assumed infinite, horizontal, and of isotropic reflectance. In practice, however, the reflected irradiance incident on PV panels outside of the front row would be overestimated with this approach.

The main difficulty is the computation of the sky diffuse irradiance, which has been studied by many authors with different approaches ranging from the simplest isotropic model to more elaborated and complex formulations (Gueymard 1987; Kambezidis et al. 1994; Khalil and Shaffie 2013; Liu and Jordan 1960; Loutzenhiser et al. 2007; Muneer and Saluja 1985; Olmo et al. 1999; Padovan and Del Col 2010; Wattan and Janjai 2016; Xie and Sengupta 2016). A review of these models, to which the reader is referred, appeared recently (Yang 2016). Based on the existing studies of the literature, one of the most widely used and validated models is the Perez model (Perez et al. 1987, 1988, 1990). It is the result of a detailed analysis of the isotropic diffuse, circumsolar, and horizon brightening irradiances that are computed by using empirically derived parameters. This approach works well with hourly data but has been recently found to generate erroneous values with sub-hourly data when $\mathrm{Kt}>1$ (i.e., under cloud-enhancement conditions) (Gueymard 2017).

\subsection{Introduction to Satellite-Based Models}

The goal of satellite-based irradiance models is to use observed information about top of atmosphere (TOA) upwelling radiances and surface albedos to derive GHI and DNI at the surface of the Earth. During the last decades, satellite-based retrievals of GHI have been usedfor example, for climate studies (Justus et al. 1986). A broad overview of these methods was published by Renné et al. (1999). These methods were originally divided into subjective, empirical/statistical, empirical/physical, and physical methods (Pinker et al. 1995; Schmetz, 1989; Myers 2013). The empirical/statistical methods are based on developing relationships between satellite- and ground-based observations; the empirical/physical and theoretical methods estimate surface radiation directly from satellite information using retrieval schemes to determine the atmospheric properties important to radiative transfer. Most empirical/statistical and empirical/physical models are now classified as semi-empirical because they involve the development of intermediate relationships either to relate satellite observations with surface radiation measurements or to convert satellite observations directly to solar radiation estimates. Empirical and semi-empirical methods generally produce only GHI and require additional models (see Section 4.2) to calculate DNI from GHI.

\subsubsection{Geostationary Satellites}

Geostationary satellites located above the equator that orbit at the same rate as the Earth's rotation provide continuous coverage of their field of view (FOV). Observations are usable up to $60^{\circ}$ north and south latitudes because of the Earth's curvature, as can be seen in Figure 4-1. The Geostationary Operational Environmental Satellite (GOES) series covers North and South America every 3 hours and the Northern Hemisphere, including the United States, every 30 minutes. Two GOES satellites (GOES-East, or GOES-13; and GOES-West, or GOES-15) operate concurrently and provide 30-minute coverage for the entire United States. The imager 
on the current GOES satellites makes radiance observations at 5 wavelength bands, or spectral regions. The visible channel $(0.64 \mu \mathrm{m})$ has a nominal 1-km resolution; the infrared channels $(3.9$ $\mu \mathrm{m}, 6.5 \mu \mathrm{m}, 10.7 \mu \mathrm{m}$, and $12 \mu \mathrm{m})$ have 4-km resolution. GOES-16 was launched in November 2016 and is expected to replace GOES-13 as the GOES-East satellite. GOES-16 is the first of the next series of GOES satellites and has a new instrument called the Advanced Baseline Imager (ABI), with 5-minute coverage at 1-km resolution for 16 channels (including 6 in the visible and near-infrared).

The European Organization for the Exploitation of Meteorological Satellites Union (EUMETSAT) owns the METEOSAT series of satellites that covers Europe and Africa, as well as the Indian Ocean and western Asia. The visible and infrared imager on the METEOSAT First Generation (MFG) satellites (up to METEOSAT-7) had 3 visible channels, water vapor (6.2 $\mu \mathrm{m})$, and infrared. The visible channel produced 8-km nadir resolution; the infrared channel's nadir resolution was $5 \mathrm{~km}$. Imagery had a repetition frequency of 30 minutes. The Spin Enhance Visible and Infrared Imager on the METEOSAT Second Generation (MSG) satellites (METEOSAT-8 onward) provide satellite imagery every 15 minutes at a nominal 3-km resolution for 11 channels (Schmetz et al. 2002). The 12th channel, a high-resolution visible channel, has a nadir resolution of $1 \mathrm{~km}$.

The Himawari-8 is a third-generation satellite similar to GOES-16 and EUMETSAT's METEOSAT Third Generation (MTG) satellites and covers East Asia and the Western Pacific. Himawari-8 was launched in October 2014 and harbors the Advanced Himawari Imager (AHI), which has similar characteristics as the ABI (Besho et al. 2016). Of the 16 bands, the visible and near-infrared bands measure at $0.5-$ or $1-\mathrm{km}$ resolution, whereas the infrared bands measure at 2$\mathrm{km}$ resolution. A full disk image is produced every 10 minutes, and the sectors are generated every 2.5 minutes. Himawari-8 replaced the Multifunction Transport Satellite (MTSAT) series of satellites, which had been in operation since 2005 .

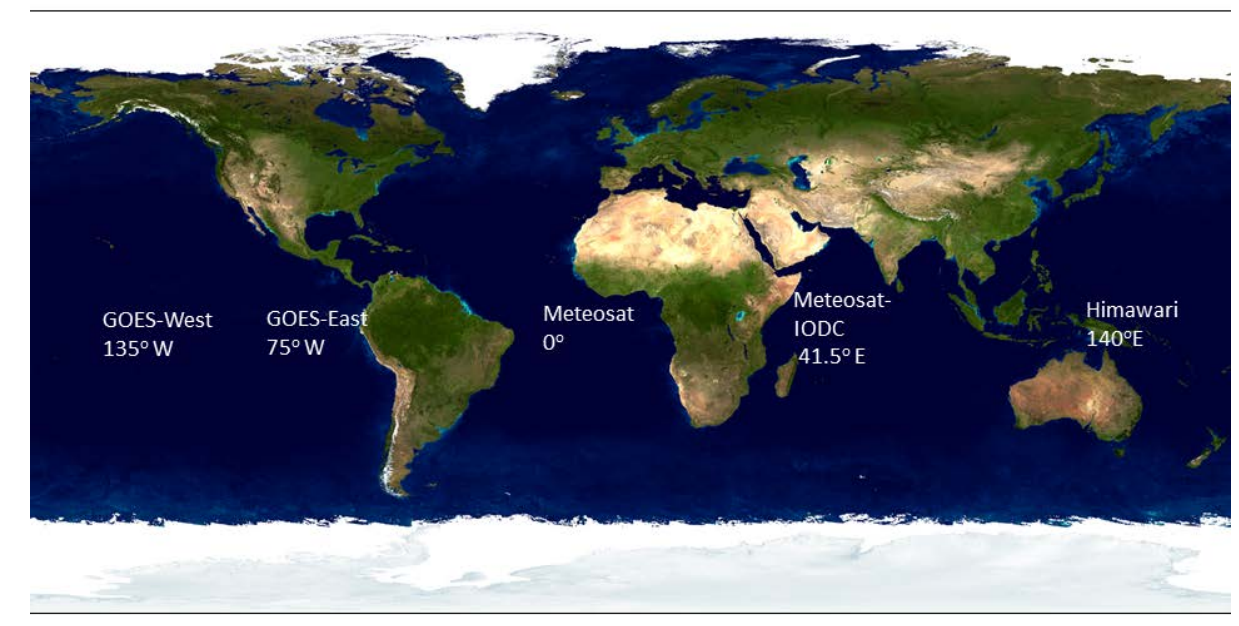

Figure 4-1. Location of the current geostationary satellites that provide coverage around the globe. Image from NASA (https://eoimages.gsfc.nasa.gov/images/imagerecords/57000/57730/land ocean ice 2048.png) 


\subsubsection{Polar-Orbiting Satellites}

Polar-orbiting satellites are used to continuously sense the Earth and retrieve cloud properties and solar radiation at the surface. An example of one such instrument is the Advanced Very High Resolution Radiometer (AVHRR) on the NOAA series of polar-orbiting platforms. Another example is the Moderate Resolution Imaging Spectroradiometer (MODIS) instrument on NASA's Aqua and Terra satellites. The Joint Polar Satellite System (JPSS) series of satellites are expected to replace the legacy NOAA polar satellites. The first satellite of the JPSS series was launched in 2011 and is called the Suomi National-Polar-Orbiting Partnership (Suomi NPP). This next-generation series of satellites has multiple instruments including the Visible Infrared Imaging Radiometer Suite (VIIRS), Ozone Mapping and Profile Suite (OMPS), and Cloud and Earth's Radiant Energy System (CERES). Although polar orbiters provide global coverage, their temporal coverage is limited because of their orbit, in which they essentially cover a particular location only once per day at the lower latitudes. In higher latitudes, a combination of many polar-orbiting-satellite-based products is recommended to achieve a sufficient temporal resolution while benefiting also from the larger spatial resolution.

\subsubsection{Satellite-Based Semi-Empirical Methods}

Satellite-based semi-empirical methods consider a pseudo-linear correlation between the atmospheric transmittance and the radiance sensed by the satellite. Semi-empirical models are classified as such because of their hybrid approach to retrieving surface radiation from satellite observations, in which the normalized satellite-observed reflectance is related to GHI at the surface. Cloud-cover indices that use visible satellite imagery are first created with budget equations between TOA and surface radiation. Those indices are then used to modify the clearsky GHI and estimate GHI at the ground consistent with the cloud scene. DNI can then be derived from GHI and the clear-sky DNI using one of the empirical methods discussed in Section 4.2. The semi-empirical approach was originally designed to create regression relationships between what is simultaneously observed by a satellite and ground-based instruments (Cano et al. 1986; Hay et al. 1978; Justus et al. 1986; Tarpley 1979). The method developed by Cano et al. (1986) is called the Heliosat method. It has been regularly updated since then and modified to rely on atmospheric transmittance properties of water vapor and aerosols to provide solar radiation estimates under clear-sky conditions, rather than direct empirical relationships with ground data.

The original Heliosat method evaluates the clearness index $\mathrm{Kt}$, or the ratio of the radiative flux at the top of the atmosphere (which is known) and the radiative flux at the Earth's surface, using the relationship:

$$
\mathrm{Kt}=\mathrm{an}+\mathrm{b}
$$

where $\mathrm{n}$ is the cloud index defined as

$$
\mathrm{n}=\left[\rho-\rho_{\mathrm{g}}\right] /\left[\rho_{\text {cloud }}-\rho_{\mathrm{g}}\right]
$$

where $\rho, \rho_{\text {cloud }}$, and $\rho_{\mathrm{g}}$ are the satellite-based reflectance observation of the current scene, of the brightest clouds, and of the ground, respectively. The cloud index is close to 0 when the observed reflectance is close to the ground reflectance (i.e., when the sky is clear). It can be 
negative if the sky is very clear, in which case $\rho$ is smaller than $\rho_{\mathrm{g}}$. The cloud index increases as clouds appear and can be greater than 1 for clouds that are optically thick.

The parameters a and $\mathrm{b}$ in Equation 4-1 can be derived empirically by comparison with coincident ground measurements or determined based on the physical principles of atmospheric transmittance, which includes not only the cloud index but also the influence of aerosol, water vapor, and trace gases. Diabaté et al. (1989) observed that three sets of parameters for the morning, noon, and afternoon were needed for Europe. The Heliosat method (and all cloudindex-based methods) requires the determination of cloud-free and extremely high cloud reflectivity instances to establish bounds to Equation 4-1. Espinar et al. (2009) and Lefèvre et al. (2007) found that a relative error in the ground albedo related to errors in determining the reflectivity from a cloud-free pixel leads to a relative error of the same magnitude in GHI under clear-sky, which corresponds to about $10 \%$ of the GHI in cloudy cases. The error due to an error in the limit for the albedo of brightest clouds increases as cloud optical thickness increases, and the relative error in the GHI can reach 60\% (Espinar et al. 2009; Lefèvre et al. 2007).

Beyer et al. (1996) developed an enhanced version of Heliosat called Heliosat-1. One major enhancement was the adoption of the clear-sky index $\mathrm{Kt}^{*}$ (ratio of the actual GHI to the GHI if the sky were clear) instead of the clearness index Kt. This resulted in the relationship $\mathrm{Kc}=1-\mathrm{n}$, which simplified the use of the method. Additional work was done to remove the dependence of the satellite radiance based on the sun-to-satellite geometry, thereby leading to a more spatially homogeneous cloud index. In addition, the determination of ground albedo and cloud albedo was improved by Beyer et al. (1996). Rigollier et al. (2004) developed Heliosat-2, which further enhanced Heliosat-1 by removing parameters that needed to be tuned and replacing them with either constants or values that can be computed automatically during the process. The HelioClim-3 and Solar Energy Mining (SOLEMI) databases, produced by MINES ParisTech/Armines and DLR, respectively, use Heliosat-2. The Heliosat-3 version was designed collaboratively by the University of Oldenburg, MINES ParisTech, and DLR, among others, and uses the SOLIS clear-sky model, which approximates radiative transfer equations for fast implementation (Mueller et al. 2004). Centro de Investigaciones Energéticas, Medioambientales y Tecnológicas (CIEMAT) and its spinoff, IrSoLaV, performed remarkable modifications on the Heliosat-3 scheme. This resulted in a different model, which includes a clear-sky detection algorithm, different possible clear-sky models with atmospheric component data sets as input, and a dynamic model for estimating the ground albedo as a function of the scattering angle (Polo et al. 2012, 2013).

Hay et al. (1978) developed a regression model that relates the atmospheric transmittance to the ratio of incoming to outgoing radiation at TOA. The transmittance was then used to derive GHI. In this method, the coefficients of the regression model change significantly based on location and need to be trained with surface observations (Nunez 1990) to produce accurate results. The Tarpley (1979) method also used the well-known relation between surface radiation, TOA radiation (both upwelling and downwelling), and atmospheric transmittance to create three separate regression equations. The regression equations were classified based on sky conditions labeled as clear, partly cloudy, and cloudy and were used accordingly.

Models such as those developed by Perez et al. (2002), Rigollier et al. (2004), and Cebecauer and Suri (2010) evolved from Cano et al. (1986) and included refinements to address albedo issues, 
when the surface is covered by snow, and the effects of sun-satellite geometry. The model has since been modified to include the use of the simplified SOLIS model (Ineichen 2008) and is used to estimate GHI first and then DNI after component separation.

\subsubsection{Satellite-Based Physical Models}

Physical models generally use radiative transfer theory to directly estimate surface radiation based on first principles, using cloud properties, water vapor, aerosol optical depth, and ozone as inputs. The radiative transfer models can be classified as either broadband or spectral, depending on whether the radiative transfer calculations involve a single broadband calculation or multiple calculations in different wavelength bands.

The broadband method of Gautier et al. (1980) used thresholds depending on multiple days of satellite pixel measurements to determine clear and cloudy skies. Separate clear-sky and cloudysky models were then used to evaluate the surface DNI and GHI. The clear-sky model initially included water vapor and Rayleigh scattering but progressively added ozone (Diak and Gautier 1983) and aerosols (Gautier and Frouin 1984). Assuming that attenuation caused by the atmosphere does not vary from clear to cloudy conditions, Dedieu et al. (1987) created a method that combines the impacts of clouds and the atmosphere. This method uses a time series of images to determine clear-sky periods for computing surface albedo. Darnell et al. (1988) created a parameterized model to calculate surface radiation using a product of the TOA irradiance, atmospheric transmittance, and cloud transmittance. Developed with data from polar-orbiting satellites, this model used collocated surface and satellite measurements to create relationships between cloud transmittance and planetary albedo.

Möser and Raschke (1983) created a model based on the premise that GHI is related to fractional cloud cover and used it with METEOSAT data to estimate solar radiation over Europe (Möser and Raschke 1984). The fractional sky cover was determined to be a function of satellite measurements in the visible channel. This method uses radiative transfer modeling (Kerschegens et al. 1978) to determine the clear- and overcast-sky boundaries. Stuhlmann et al. (1990) have since enhanced the model to include elevation dependence and additional constituents as well as multiple reflections in the all-sky model.

An important spectral model developed by Pinker and Ewing (1985) divided the solar spectrum into 12 intervals and applied the Delta-Eddington radiative transfer (Joseph et al. 1976) to a three-layer atmosphere. The primary input to the model is the cloud optical depth, which can be provided from various sources. This model was enhanced by Pinker and Laszlo (1992) and used in conjunction with cloud information from the International Satellite Cloud Climatology Project (ISCCP) (Schiffer and Rossow 1983). Another physical method involves the use of satellite information from multiple channels to derive cloud properties (Stowe et al. 1999) and then evaluate DNI and GHI using the cloud properties in a radiative transfer model. This method, called CLOUDS, was originally developed using the polar-satellite data from the AVHRR instrument onboard NOAA satellites. This method has then been modified and enhanced to use cloud properties from the GOES satellites (Heidinger 2003; Pavlonis et al. 2005). The cloud information can then be input to a radiative transfer model such as the Fast All-sky Radiation Model for Solar applications (FARMS) (Xie et al. 2016) to calculate GHI and DNI such as has been done for the development of the most recent versions of the National Renewable Energy Laboratory's (NREL's) gridded National Solar Radiation Data Base (NSRDB (1998-2015)). 
Another cloud retrieval scheme called AVHRR Processing scheme Over cLouds, Land, and Ocean (APOLLO) was developed by Kriebel et al. $(1989,2003)$ for the AVHRR instrument. APOLLO has been adapted for use with data obtained from the SEVIRI instrument on the MSG satellite. APOLLO-derived cloud products, including cloud optical depth and cloud type, can be used in a radiative transfer model such as Heliosat-4 (Oumbe et al. 2009; Qu et al. 2016), as made operational by the Copernicus service (http://www.copernicus-atmosphere.eu).

The ISCCP (Schiffer and Rossow 1983) was established in 1982 as part of the World Climate Research Programme (WCRP). The ISCCP cloud products include cloud optical thickness, cloud top temperature, cloud particle size, and other cloud properties that may be used to derive surface radiation.

Physical models are computationally more intensive than empirical and semi-empirical models. An advantage of physical models, however, is that they can use additional channels from new satellites (such as MSG or GOES-16) to improve cloud property retrieval and can include physical properties of aerosols and other gaseous species, such as water vapor, explicitly.

\subsection{Clear-Sky Models Used in Operation Models}

\subsubsection{Bird Clear-Sky Model}

The Bird clear-sky model (Bird and Hulstrom 1981) is a broadband algorithm that produces estimates of clear-sky direct beam, hemispherical diffuse, and total hemispherical solar radiation on a horizontal surface. The model uses a parameterization based on radiative transfer computations and is composed of simple algebraic expressions. Model results are expected to agree within $\pm 10 \%$ with detailed high-resolution spectral or broadband physics-based radiative transfer models. The model can be used at 1-minute or better resolutions and can duly accept inputs at that frequency if available. However, in the absence of high temporal resolution input parameters, climatological or annual average values can alternatively be used as inputs to the model. The Bird clear-sky model also forms the basis of the clear-sky part of METSTAT, with only minor modifications. The performance of these two models has been assessed rigorously and compared to other algorithms (Badescu et al. 2012; Gueymard 1993, 2003a, 2003b, 2004a, 2004b, 2012; Gueymard and Myers 2008; Gueymard and Ruiz-Arias 2015).

\subsubsection{ESRA Model}

The ESRA model is another example of a clear-sky model. Used in the HELIOSAT-2 model that retrieves GHI from satellites, this model computes DNI, GHI, and DHI using Rayleigh optical depth, elevation, and the Linke turbidity factor as its inputs. The performance of the model has been evaluated at various locations (Badescu et al. 2012; Gueymard and Myers 2008; Gueymard 2012; and Gueymard and Ruiz-Arias 2015).

\subsubsection{SOLIS Model}

The SOLIS model (Mueller et al. 2004) is a relatively simple spectral clear-sky model that can calculate DNI, GHI, and diffuse radiation based on an approximation to the Lambert-Beer relation for computing DNI:

$$
\mathrm{I}=\mathrm{I}_{0} \mathrm{e}^{\left(-\mathrm{M}^{*} \tau\right)}
$$


where

- $\tau$ is the atmospheric optical depth at a specific (monochromatic) wavelength

- $\mathrm{M}$ is the optical air mass

- $\mathrm{I}_{0}$ is the TOA direct irradiance

- I is the DNI at the surface for a monochromatic wavelength.

This equation is modified to account for slant paths and adapted for GHI and diffuse. The modified Lambert-Beer relation (Mueller et al. 2004) is

$$
\mathrm{I}(\mathrm{SZA})=\mathrm{I}_{0} \mathrm{e}^{-\tau 0 / \operatorname{Cos}(\mathrm{SZA})}
$$

where

- $\mathrm{I}(\mathrm{SZA})$ is the irradiance associated with the empirical factor, $a$, used to compute DNI, DHI, or GHI ( $a=1$ for DNI)

- $\tau_{0}$ is the vertical broadband optical depth of the atmosphere

- $\quad$ SZA is the solar zenith angle.

The Beer-Lambert equation is a simple relationship because it accounts for monochromatic DNI and is impacted only by atmospheric attenuation. On the other hand, DHI and GHI are broadband values that contain energy that is scattered by the atmosphere. The empirical factor $a$ is used as an adjustment factor to compute either GHI or DHI, as explained in Mueller et al. (2004). Ineichen (2008) developed a simplified (broadband) version of that clear-sky model by developing parameterizations to replace radiative transfer model runs, thereby increasing the speed of the model.

\subsubsection{McClear Model}

The fast clear-sky broadband model called McClear implements a fully physical model, replacing the empirical relations or simpler models used before, such as ESRA. It exploits the recent results on aerosol properties and total column content in water vapor and ozone produced by the European Copernicus Atmospheric Monitoring Service (CAMS) project. It is based on abacci pre-computed with the radiative transfer model libRadtran. McClear irradiances were compared to 1-minute measurements made under clear-sky conditions at several Baseline Surface Radiation Network (BSRN) stations representative of various climates (Lefèvre et al. 2013). For GHI and DNI, the correlation coefficients range from $0.95-0.99$ and $0.86-0.99$, respectively. The bias ranges from $14-25 \mathrm{~W} / \mathrm{m}^{2}$ and $49-+33 \mathrm{~W} / \mathrm{m}^{2}$, respectively. The root mean square error (RMSE) ranges from $20 \mathrm{~W} / \mathrm{m}^{2}$ (3\% of the mean observed irradiance) to $36 \mathrm{~W} / \mathrm{m}^{2}$ $(5 \%)$, respectively $33 \mathrm{~W} / \mathrm{m}^{2}(5 \%)$ to $64 \mathrm{~W} / \mathrm{m}^{2}(10 \%)$.

\subsubsection{REST2 Model}

The high-performance REST2 model is based on transmittance parameterizations over two distinct spectral bands separated at $0.7 \mu \mathrm{m}$. The model's development and its benchmarking are described by Gueymard (2008b). REST2 has been thoroughly validated and compared to other irradiance models under varied atmospheric conditions, including extremely high aerosol loads 
(Antonanzas-Torres et al. 2016; Engerer and Mills 2015; Gueymard 2012, 2014; Gueymard and Myers 2008; Gueymard and Ruiz-Arias 2015; Sengupta and Gotseff 2013; Zhong and Kleissl 2015).

The model is used in solar-related applications, including the benchmarking of the radiative output of the Weather Research and Forecasting (WRF) model (Ruiz-Arias et al. 2012), operational derivation of surface irradiance components using MODIS satellite observations (Chen et al. 2014), improvement in GHI to DNI separation modeling (Vindel et al. 2013), and development of future climate scenarios (Fatichi et al. 2011). REST2 is also being used at NREL and is integrated into its suite of algorithms that produces the current version of the NSRDB (1998-2015).

\subsection{All-Sky Models used in Operational Models}

\subsubsection{FARMS}

Radiative transfer models are capable of simulating atmospheric radiation under all-sky conditions and have been used in a broad range of applications, such as satellite remote sensing or climate studies. Compared to other applications, solar energy has unique requirements from radiative transfer models and thus has particular prerequisites in the model design. For instance, the study of solar energy demands more efficient simulations of solar irradiance than the conventional models used in weather or climate studies, such as the Rapid Radiation Transfer Model (RRTM) or its simplified version for inclusion on general circulation models (RRTMG). To provide a new option for efficiently computing solar radiation, NREL has developed FARMS (Xie et al. 2016) using cloud transmittances and reflectances for direct and diffuse radiation computed by RRTM with the 16-stream discrete-ordinates radiative transfer method. To reduce the computing burden, the cloud transmittances and reflectances are parameterized as functions of solar zenith angle (SZA), cloud thermodynamic phase, optical thickness, and particle size. The all-sky GHI, DHI, and DNI are ultimately computed by coupling the cloud transmittances and reflectances with surface albedo and a fast clear-sky radiation model (REST2) to account for the atmospheric absorption and scattering.

To understand the accuracy and efficiency of FARMS, GHI was simulated using the cloud microphysical and optical properties retrieved from GOES data during 2009-2012 with both FARMS and RRTMG (a two-stream radiative transfer model) and compared to measurements taken from the U.S. Department of Energy's Atmospheric Radiation Measurement Climate Research Facility Southern Great Plains site. Results indicate that the accuracy of FARMS is comparable to or better than the two-stream approach; however, FARMS is approximately 1,000 times more efficient and faster because it does not explicitly solve the radiative transfer equation for each individual cloud condition.

Note that FARMS, as well as the conventional radiative transfer models developed for weather and climate studies, only outputs broadband irradiance over horizontal surfaces. Further efforts to improve FARMS are on the way to expand its capabilities to incorporate tilted surfaces and spectral distributions. 


\subsubsection{Heliosat}

The CAMS radiation service uses a physical retrieval of cloud parameters and the fast parameterized radiative transfer method called Heliosat-4 (Qu et al. 2016). The new Heliosat-4 method computes GHI, DNI, and DHI under all-sky conditions as a broadband aggregation of spectrally resolved internal computations. It is a fast but accurate physical model that mimics a full radiative transfer model and is well suited for geostationary satellite retrievals. The method is based on the work of Oumbe et al. (2014), which proved that the surface solar irradiance can be approximated by the product of the irradiance under clear conditions (cloud free) and a modification index only depending on cloud properties and ground albedo. It the reason why Heliosat-4 contains two precomputed look-up-table-based models, the McClear model (Lefèvre et al. 2013) for clear-sky conditions and the McCloud model for cloudy conditions. The databases for both models were developed using the libRadtran radiative transfer model (Mayer and Kylling 2005). The main inputs to McClear are aerosol properties, total column water vapor, and ozone, whereas cloud properties, such as cloud optical depth, are the main inputs to the McCloud part of Heliosat-4. In the case of MSG satellites, cloud properties are derived at a 15minute temporal resolution using an adapted APOLLO retrieval scheme (see Section 5.3.1). The Heliosat- 4 method is based on decoupling clear and cloudy scenes, as proposed by Oumbe et al. (2014), where surface radiation at the surface can be approximated by the product of the irradiance under clear conditions and a modification factor due to cloud properties and ground albedo. An easy-to-read summary can be found in the "User's Guide to the CAMS Radiation Service"1 (Schroedter et. al. 2016).

\subsection{NWP-Based Solar Radiation Estimates}

NWP models, run in either reanalysis mode or when providing weather forecasts, are capable of providing GHI estimates for long periods of time. The accuracy of such estimates is known to be lower than those provided by the satellite-based models. Significant improvements, however, can be obtained in such estimates by improving both model physics and assimilation of various observations. Some of the commonly available models and data sets are described in what follows. It should be noted that this is not a complete and comprehensive list. The goal here is only to provide the user with initial information related to this potential source of data.

\subsubsection{Reanalysis Models}

The ERA-Interim is a global atmospheric reanalysis that provides data starting in 1979. This data set is produced using ECMWF's 2006 version of the data assimilation system used in the IFS.

This system uses four-dimensional variational analysis (4D-Var) and provides analysis data with TOA and global horizontal irradiations (all-sky and clear-sky) every 6 hours on an approximately $1^{\circ} \times 1^{\circ}$ grid. More information can be found at ECMWF's website ${ }^{2}$.

NASA's MERRA-2 is another global atmospheric reanalysis data set that provides data starting in 1980 and comprises TOA and global horizontal irradiations (all-sky and clear-sky). It includes additional data sets from what was assimilated into the original MERRA data set. The spatial resolution is $0.5^{\circ} \mathrm{x} 0.625$ and temporal resolution is hourly.

\footnotetext{
${ }^{1}$ See http://www.sodapro.com/documents/10157/326238/CAMS72_2015SC1_D72.11.3.1_201612_UserGuide_v2.pdf/ed54f8ec-e19e4948-af14-d4c2a94083ac.

${ }^{2}$ https://www.ecmwf.int/
} 
Finally, the Climate Forecast System Reanalysis (CFSR) from NOAA provides reanalysis data from 1979. The data are available hourly at $0.5^{\circ}$ resolution.

\subsubsection{Forecast Models}

Various national meteorological agencies run operational weather forecasts both regionally and globally. Some of the data from these operational models may be available from archives. Some of the most popular examples of global data sets are from ECMWF's IFS runs and from NOAA's Global Forecast System (GFS) runs. There are various regional model runs by national meteorological agencies that produce forecasts for individual countries and regions. Because many data sets now exist, this source of data is mentioned without pointing to specific sources. See Section 8.2.3.1 for additional information and some examples of such data sets.

\subsection{Site Adaptation: Merging Measurements and Models}

A major goal of solar resource assessments is to provide high-quality data to evaluate the financial viability of solar power plant projects. This essentially implies that accurate data over long time periods are available for conducting these studies. Normally, satellite-derived data time series fulfill the requirement for long-term data. However, they may be hampered by inherent bias and uncertainty because of the following:

1. The information content, quality, and spatial and temporal resolution of the raw satellite data

2. The approximations made by the models converting satellite observations into surface solar radiation estimates

3. The uncertainty in ancillary information needed by these models.

As part of a resource assessment study for a new solar power plant, ground-based solar measurements are conducted for a short period of time (nominally about 1 year) and used to validate the satellite data. The main goal is actually to remove some of the uncertainties and bias in those data sets. This process has been given various names, including "site adaptation," which is used here for simplification. A review paper by Polo et al. (2016) provides a summary of the various methods currently used. It should be noted, however, that the ground-based irradiance data need to be of high quality, otherwise the correction method could actually degrade the quality of the modeled time series. High-quality ground measurements can only be achieved through the use of well-calibrated, high-quality instruments that have been deployed at wellchosen locations, using optimal installation methods and regular maintenance, per the best practices described in other sections.

Site-adaptation methods can be separated into two broad categories. The first consists of physical methods that attempt to reduce the uncertainty and bias in the data by improving the satellite model inputs, such as AOD. The second approach develops statistical correction schemes directly comparing the satellite-based irradiance estimates with "unbiased" ground observations and uses those functions to correct the satellite-based radiation estimates. Additional information about site-adaptation models are provided in Chapter 8. 


\subsubsection{Physical Methods}

As the highest uncertainty in satellite models is in DNI, the primary goal is to reduce errors in DNI by improving the quantification of AOD. Methods such as those proposed by Gueymard $(2011,2012)$ demonstrate how accurate AOD data obtained from ground sunphotometric measurements can indeed improve DNI. Nevertheless, the scarcity of such high-quality AOD observations implies that other sources should be used. Possible sources of AOD with global coverage include retrievals from the MODIS and MISR satellites, data assimilation output from CAMS, and NASA's MERRA-2 data. In parallel, specific methods have been developed by Gueymard and Thevenard (2009) and Ruiz-Arias et al. (2013a, 2013b) to correct bias and uncertainty in the satellite- or model-based AOD data using ground observations. These adjusted AOD data sets have been shown to improve the satellite-based solar radiation estimates at various locations.

\subsubsection{Statistical Methods}

Various statistical methods have been developed to use short-term ground measurements to directly correct long-term satellite-based data sets. These bias correction methods range from linear methods (Cebecauer and Suri 2010; Vindel et al. 2013; Harmsen et al. 2014; Polo et al. 2015) to various nonlinear methods including feature transformation (Schumann et al. 2011), polynomial-based corrections (Mieslinger et al. 2013), model output statistics (MOS) corrections (Bender et al. 2011; Gueymard et al. 2012), measure-correlate-predict (MCP) correction (Thuman et al. 2012), and Fourier-decomposition-based corrections (Vernay et al. 2013). Other statistical methods include regional fusion methods of ground observations with satellite-based data (Journée et al. 2012; Ruiz-Arias et al. 2015) and improvements to the irradiance cumulative distribution function (Cebecauer and Suri 2012; Blanc et al. 2012).

\subsection{Summary}

This chapter provided a brief overview of solar radiation modeling methods with a focus on satellite-based models. Since the 1980s, both the technology of operational meteorological satellites and models to estimate surface radiation from these satellites have improved in their resolution and accuracy. With the recent launch of GOES-16, the world is now mostly covered at 15-minute temporal resolution or better and 1-km spatial resolution. Improvements in computational capabilities have also contributed to improve our ability to use increasingly sophisticated models that can use higher volumes of satellite and ancillary data sets and ultimately deliver products of increasing resolution and accuracy.

This chapter also contained a short introduction to NWP modeling as improvements in that area can contribute to better irradiance estimates around the globe. This chapter has been kept deliberately short, while providing the interested readers with references for more detailed reading. Finally, the appendix below provides short descriptions of some of the satellite-based data sets that are commonly used.

\section{Appendix}

\section{Some Currently Available Satellite-Based Data Sets}

The following sections present examples of currently available operational models. Only a selection of models is presented here. Further public, scientific, and commercial operational models exist and might also be of interest for solar resource analyses. 


\section{NSRDB PSM (2015 Update)}

For many years, NREL has maintained a ground-based solar radiation data set known as the NSRDB. This data set included both actual in-situ ground measurements and the METSTAT model (Maxwell et al. 1997) to convert U.S. National Weather Surface ground-based sky observations to solar radiation estimates. The original NSRDB (1961-1990) (NREL 1992) covered the period 1961-1990 for 239 ground stations in the United States. This version of the NSRDB (1991-2005) was subsequently updated, adding in many more ground stations and making use of satellite-based data to correct for some of the ground-based measurements (NREL 2007). Now, in collaboration with the University of Wisconsin and NOAA, NREL has produced a physics-based satellite-derived solar radiation data set as part of a new gridded NSRDB (19982015). This gridded NSRDB (1998-2015) uses the Physical Solar Model (PSM) that produces satellite-based data every 30 minutes for 4-km-resolution pixels for North and South America and is freely available from the NSRDB website (https://nsrdb.nrel.gov). The data fields include solar radiation and meteorological data.

The PSM uses a two-stage scheme that retrieves cloud properties and uses those properties in a radiative transfer model to compute surface radiation. In the first stage, cloud properties are generated using the AVHRR Pathfinder Atmospheres-Extended (PATMOS-x) algorithms (Heidinger et al. 2014). In the second stage, GHI and DHI are computed by the FARMS model (Xie et al. 2016), using these cloud properties, as well as additional meteorological parameters as inputs. The FARMS model uses the REST2 model (Section 4.4.5) for clear-sky calculations and a fast all-sky model for cloudy-sky calculations (Section 4.5.1). The AOD inputs required for clear-sky calculations are obtained from the MODIS, MISR, and MERRA-2 aerosol products from NASA after scaling and bias reduction using ground AOD measurements from AERONET. Water vapor, temperature, wind speed, relative humidity, and dewpoint data are obtained from NASA's MERRA-2.

The time-series irradiance data for each pixel are quality checked to ensure that they are within acceptable physical limits, gaps are filled, and the Coordinate Universal Time (UTC )time stamp is shifted to local standard time. Finally, the GOES East and West data sets are blended to create a contiguous data set for the period from $1998-2015$.

Evaluation of the NSRDB 1998-2015 was conducted by Habte et al. (2017) to quantify the accuracy and the spatial and temporal variability of the solar radiation data by comparing to high-quality ground measurements representing various climatic regions around the United States. Table 4-1 shows a statistical summary of the evaluation. 
Table 4-1. Statistical Results of the Comparison Between the NSRDB (1998-2015) and Surface Measurements

\begin{tabular}{|c|c|c|c|c|c|c|c|c|c|}
\hline \multirow[b]{2}{*}{$\begin{array}{c}\text { Statio } \\
\mathbf{n}\end{array}$} & \multirow[b]{2}{*}{ Time Avg. } & \multicolumn{4}{|c|}{ GHI } & \multicolumn{4}{|c|}{ DNI } \\
\hline & & $\begin{array}{l}\text { No. } \\
\text { Obs. }\end{array}$ & $\begin{array}{l}\underset{3}{\text { MBE }} \\
(\%)\end{array}$ & $\begin{array}{l}\text { RMSE } \\
{ }^{4}(\%)\end{array}$ & $\begin{array}{l}M^{\prime} E^{5} \\
(\%)\end{array}$ & $\begin{array}{l}\text { No. } \\
\text { Obs. }\end{array}$ & $\begin{array}{l}\text { MBE } \\
(\%)\end{array}$ & $\begin{array}{l}\text { RMSE } \\
(\%)\end{array}$ & $\begin{array}{l}\text { MAE } \\
(\%)\end{array}$ \\
\hline \multirow{4}{*}{ BON } & Hourly & 66,685 & 1.95 & 24.35 & 21.71 & 52,803 & 8.31 & 37.12 & 32.05 \\
\hline & Daily Totals & 6,565 & 1.95 & 10.49 & 8.34 & 6,545 & 8.18 & 19.12 & 18.40 \\
\hline & MMDT* & 216 & 1.92 & 5.71 & 4.56 & 213 & 8.49 & 10.38 & 9.37 \\
\hline & AMMMDT $^{* *}$ & 18 & 1.92 & 3.51 & 2.70 & 18 & 8.46 & 8.82 & 8.46 \\
\hline \multirow{4}{*}{ DRA } & Hourly & 66,322 & -1.30 & 17.29 & 15.19 & 63,264 & -0.48 & 21.71 & 14.73 \\
\hline & Daily Totals & 6,467 & -1.30 & 5.20 & 3.75 & 6,467 & -0.50 & 9.02 & 7.41 \\
\hline & MMDT & 214 & -1.31 & 1.97 & 1.74 & 214 & -0.48 & 2.95 & 2.45 \\
\hline & AMMMDT & 18 & -1.31 & 1.54 & 1.36 & 18 & -0.49 & 1.54 & 1.11 \\
\hline \multirow{4}{*}{ TBL } & Hourly & 66,233 & -3.19 & 22.28 & 16.20 & 56,840 & -4.57 & 32.07 & 25.11 \\
\hline & Daily Totals & 6,568 & -3.19 & 11.26 & 8.31 & 6,567 & -4.58 & 15.69 & 13.45 \\
\hline & MMDT & 216 & -3.23 & 5.11 & 4.11 & 216 & -4.58 & 6.68 & 5.57 \\
\hline & AMMMDT & 18 & -3.23 & 3.86 & 3.23 & 18 & -4.58 & 5.01 & 4.58 \\
\hline \multirow{4}{*}{ FPK } & Hourly & 65,157 & -4.44 & 26.70 & 22.87 & 51,553 & 4.60 & 34.18 & 27.28 \\
\hline & Daily Totals & 6,556 & -4.44 & 13.90 & 10.40 & 6,537 & 4.18 & 15.66 & 14.19 \\
\hline & MMDT & 216 & -4.51 & 9.81 & 7.10 & 216 & 4.71 & 7.54 & 6.50 \\
\hline & AMMMDT & 18 & -4.51 & 5.36 & 4.51 & 18 & 4.71 & 5.35 & 4.71 \\
\hline \multirow{4}{*}{ GWN } & Hourly & 66,162 & 3.97 & 22.91 & 20.62 & 56,109 & 9.07 & 35.78 & 29.73 \\
\hline & Daily Totals & 6,466 & 3.97 & 8.49 & 7.01 & 6,463 & 8.90 & 18.05 & 16.78 \\
\hline & MMDT & 216 & 3.96 & 5.11 & 4.32 & 216 & 9.02 & 10.87 & 9.34 \\
\hline & AMMMDT & 18 & 3.96 & 4.83 & 3.96 & 18 & 9.02 & 9.69 & 9.02 \\
\hline \multirow{4}{*}{ SXF } & Hourly & 45,163 & 0.66 & 19.97 & 14.89 & 36,975 & 8.05 & 29.40 & 23.79 \\
\hline & Daily Totals & 4,570 & 0.66 & 12.07 & 9.10 & 4,556 & 8.23 & 17.10 & 15.38 \\
\hline & MMDT & 151 & 0.62 & 7.64 & 6.20 & 151 & 8.01 & 9.74 & 8.53 \\
\hline & AMMMDT & 13 & 0.67 & 2.23 & 1.94 & 13 & 7.70 & 8.41 & 7.76 \\
\hline \multirow{4}{*}{ PSU } & Hourly & 64,888 & 1.14 & 27.62 & 24.80 & 49,740 & 8.16 & 42.75 & 38.86 \\
\hline & Daily Totals & 6,370 & 1.14 & 10.36 & 8.90 & 6,359 & 8.02 & 20.33 & 20.56 \\
\hline & MMDT & 211 & 1.25 & 5.09 & 4.11 & 211 & 8.35 & 10.56 & 9.54 \\
\hline & AMMMDT & 18 & 1.48 & 3.95 & 2.93 & 18 & 8.45 & 9.18 & 8.45 \\
\hline \multirow{4}{*}{$\begin{array}{l}\text { NREL } \\
- \\
\text { SRRL }\end{array}$} & Hourly & 61,452 & -0.35 & 29.08 & 24.62 & 53,429 & 0.37 & 38.93 & 31.13 \\
\hline & Daily Totals & 6,217 & -0.34 & 11.57 & 8.90 & 6,217 & 0.37 & 17.74 & 14.95 \\
\hline & MMDT & 206 & -0.36 & 5.60 & 4.35 & 206 & 0.37 & 7.48 & 6.00 \\
\hline & AMMMDT & 18 & -0.47 & 3.50 & 2.92 & 18 & 0.38 & 3.64 & 2.92 \\
\hline SGP. & Hourly & 63,888 & 3.95 & 15.97 & 11.21 & 53,117 & 7.06 & 26.25 & 19.95 \\
\hline
\end{tabular}




\begin{tabular}{|c|l|cccc|cccc|}
\hline $\begin{array}{c}\text { Statio } \\
\mathbf{n}\end{array}$ & Time Avg. & $\begin{array}{l}\text { No. } \\
\text { Obs. }\end{array}$ & $\begin{array}{l}\text { MBE } \\
\mathbf{3}\end{array}$ & $\begin{array}{l}\text { RMSE } \\
\mathbf{4}(\%)\end{array}$ & $\begin{array}{l}\text { MAE } \\
(\%)\end{array}$ & $\begin{array}{l}\text { No. } \\
\text { (\%) }\end{array}$ & $\begin{array}{l}\text { MBE } \\
(\%)\end{array}$ & $\begin{array}{l}\text { RMSE } \\
(\%)\end{array}$ & $\begin{array}{l}\text { MAE } \\
(\%)\end{array}$ \\
\hline ARM & Daily Totals & 6,052 & 3.90 & 8.60 & 6.45 & 6,030 & 7.14 & 15.24 & 13.38 \\
& MMDT & 203 & 3.93 & 5.12 & 4.39 & 203 & 6.99 & 8.63 & 7.24 \\
& AMMMDT & 17 & 3.94 & 4.35 & 3.94 & 17 & 7.00 & 7.39 & 7.00 \\
\hline
\end{tabular}

* MMDT - Monthly mean daily totals

**AMMMDT - Annual mean monthly mean daily totals

\section{NASA/Global Energy and Water Cycle Experiment Surface Radiation Budget}

To serve the needs of the World Climate Research Programme, Whitlock et al. (1995) developed a global Surface Radiation Budget (SRB) data set using cloud information from the ISCCP C1 data set at a $250-\mathrm{km}$ by $250-\mathrm{km}$ (approximately $2.5^{\circ} \times 2.5^{\circ}$ ) resolution every 3 hours (Schiffer and Rossow 1983; Zhang et al. 2004). Information from the ISCCP-C1 data set is used as an input into the Pinker and Laszlo (1992) model and the Darnell et al. (1988) model.

The currently available version is the NASA/Global Energy and Water Cycle Experiment SRB Release-3.0 data sets that contains global 3-hourly, daily, monthly/3-hourly, and monthly averages of surface longwave and shortwave radiative parameters on a $1^{\circ} \times 1^{\circ}$ grid. Primary inputs to the models include:

- Visible and infrared radiances and cloud and surface properties inferred from ISCCP pixel-level (DX) data

- Temperature and moisture profiles from the GEOS-4 reanalysis product obtained from the NASA Global Modeling and Assimilation Office

- Column ozone amounts constituted by assimilating various observations.

The SRB data set is available from multiple sources. The Surface meteorology and Solar Energy (SSE) website provide SRB data in a version that is more applicable to renewable energy. ${ }^{6}$ SRB data sets are also available from the CERES project. ${ }^{7}$ Additionally, the Fast Longwave and Shortwave Radiative Fluxes (FLASHFlux) project generates real-time SRB data. ${ }^{8}$ All these projects use global observations from CERES and MODIS instruments onboard polar-orbiting satellites. Table 4-2 shows the estimated bias and RMS error between measured WMO BSRN monthly averages of the three usual solar radiation components. The NASA SSE accuracy and methodology are documented on the SSE website.

\footnotetext{
${ }_{7}^{6}$ See $h$ ttp://eosweb.larc.nasa.gov/sse/.

${ }^{7} \mathrm{See}$ https://eosweb.larc.nasa.gov/project/ceres/ceres table.

${ }^{8} \mathrm{See}$ https://eosweb.larc.nasa.gov/project/ceres/flashflux-12 table.
} 
Table 4-2. Regression Analysis of NASA SSE Compared to BSRN Bias and RMS Error for Monthly Averaged Values from July 1983-June $2006^{9}$

\begin{tabular}{|l|l|r|r|}
\multicolumn{1}{|c|}{ Parameter } & \multicolumn{1}{|c|}{ Region } & Bias (\%) & RMS (\%) \\
& GHI & -0.01 & 10.25 \\
& Global & -1.18 & 34.37 \\
& $60^{\circ}$ poleward & 0.29 & 8.71 \\
\hline DHI & $60^{\circ}$ equatorward & 7.49 & 29.34 \\
& Global & 11.29 & 54.14 \\
& $60^{\circ}$ poleward & 6.86 & 22.78 \\
\hline DNI & $60^{\circ}$ equatorward & -4.06 & 22.73 \\
& Global & -15.66 & 33.12 \\
& $60^{\circ}$ poleward & 2.40 & 20.93 \\
\hline
\end{tabular}

\section{DLR-ISIS Model}

Similar to the NASA SSE data sets discussed in Section 4.3.2, the DLR-ISIS data set ${ }^{10}$ is a 21year DNI and GHI data set ( $280 \mathrm{~km}$ by $280 \mathrm{~km}$ every 3 hours) based on the ISCCP cloud product covering the period from July 1983-December 2004. The cloud products are used in a 2-stream radiative transfer model (Kylling et al. 1995) to evaluate DNI and GHI. The correlated-k method from Kato et al. (1999) is used to compute atmospheric absorption in the solar spectrum. Scattering and absorption in water clouds are analyzed using the parameterization of $\mathrm{Hu}$ and Stamnes (1993); ice cloud properties are obtained from Yang et al. (2000) and Key et al. (2002). Fixed effective radii of $10 \mu \mathrm{m}$ and $30 \mu \mathrm{m}$ are used for water and ice clouds, respectively. The radiative transfer algorithm and parameterizations are included in the radiative transfer library libRadtran (Mayer and Kylling 2005).

The complete method for creating the DLR-ISIS data set using the ISCCP cloud products and the libRadtran library is outlined in Lohmann et al. (2006). The cloud data used for the derivation of the DLR-ISIS data set are taken from the ISCCP FD (global radiative flux data product) input data set (Zhang et al. 2004), which is based on ISCCP D1 cloud data. (See the ISCCP homepage for more information about cloud data sets. ${ }^{11}$ ) It provides 3 -hour cloud observations on a $280-\mathrm{km}$ by $280-\mathrm{km}$ equal area grid. The whole data set consists of 6,596 grid boxes on 72 latitude steps of $2.5^{\circ}$. This grid is maintained for the DLR-ISIS data set.

ISCCP differentiates among 15 cloud types. The classification includes three intervals of optical thickness in three cloud levels: low, middle, and high clouds. Low and middle cloud types are further divided into water and ice clouds; high clouds are always ice clouds.

For DLR-ISIS, optical thickness, cloud top pressure, and cloud phase given in the ISCCP data set are processed to generate clouds for the radiative transfer calculations. One radiative transfer calculation is carried out for each occurring cloud type assuming $100 \%$ cloud coverage, plus one

\footnotetext{
${ }^{9}$ From https://eosweb.larc.nasa.gov/cgi-bin/sse/sse.cgi? + s06\%23s06.

${ }^{10}$ See http://www.pa.op.dlr.de/ISIS/.

${ }^{11} \mathrm{See}$ http://isccp.giss.nasa.gov.
} 
calculation for clear sky. For the final result, irradiances are weighted with the cloud amount for each cloud type and for clear sky, respectively.

\section{HelioClim}

The Heliosat-2 method based on Cano et al. (1986) and modified by Rigollier et al. (2004) is used to produce the HelioClim databases ${ }^{12}$ using METEOSAT data. The HelioClim databases cover Europe, Africa, the Mediterranean Basin, the Atlantic Ocean, and part of the Indian Ocean (latitude and longitude between $\pm 66^{\circ}$ ). The freely available HelioClim-1 database established from MFG covers the period ranging from 1985-2005 and provides daily values of GHI with a spatial resolution of $25 \mathrm{~km}$. Some statistical comparison analyses with ground measurements have been provided by Blanc et al. (2011).

The two current versions of the HelioClim-3 database (versions 4 and 5) are based on MSG and provide, over its FOV, 15-min surface solar irradiance estimates with a spatial resolution of $3 \mathrm{~km}$ at nadir. These databases are available for free for the period ranging from February 2004December 2006. Transvalor, the valorization company of MINES ParisTech/ARMINES, commercializes, through their website www.soda-pro.com, the two HelioClim-3 databases for 2007 onward. The version 4 of the database makes use of the ESRA clear-sky irradiance model with the climatological database of monthly values of Linke turbidity (Remund et al. 2003). This database is able to provide surface solar irradiance estimates on near real-time basis, with few minutes of delay after the last image acquisition by MSGn every 15 minutes. The version 5 of HelioClim-3 makes use of the McClear clear-sky irradiance model and provides estimates with a delay of 2 days.

Ineichen (2016) provided an independent validation of HelioClim-3 versions 4 and 5, and comparisons statistics notably with BSRN stations can be found here: http://www.sodapro.com/help/helioclim/helioclim-3-validation/bsrn-stations.

\section{Solar Energy Mining}

SOLEMI is a service from DLR that provides irradiance data commercially and for scientific purposes. The data are based on global atmospheric data sets (aerosol, water vapor, ozone) from different earth observation sources and climate models as well as cloud data from Meteosat. GHI and DNI data sets are available every hour at a $2.5-\mathrm{km}$ resolution and cover Europe and Africa (1991-2012) and Asia (1999-2012). SOLEMI uses the Heliosat-2 method of Rigollier et al. (2004).

\section{MACC-RAD Services}

Within the European Commission's Copernicus program, CAMS provides atmospheric composition as aerosols, water vapor, and ozone. By coupling with MSG satellite-based cloud physical parameters in the Heliosat-4 method, the CAMS radiation service provides clear-sky and all-sky global, direct, diffuse, and direct normal irradiation. The service is jointly provided by DLR, Armines, and Transvalor with help of the SOlar radiation DAta (SODA) service.

Besides all-sky irradiation, also clear-sky (cloudless) irradiation is proved as the CAMS McClear service. Both services provide time series with a temporal resolution of 1 minute, 15 minutes, 1

\footnotetext{
${ }^{12}$ See http://www.soda-is.com/eng/helioclim/heliosat.html.
} 
hour, 1 day, or 1 month at the latitude and longitude requested by the user. Time series may be accessed by an interactive user interface or automatically in a scripting environment. The data records start in 2004 and last until present time. Data are continuously updated and provided with up to 2 days delay. The coverage is on the global scale for CAMS McClear and in Europe/Africa/Middle East for the CAMS all-sky radiation service. A verbose mode allows access to all used atmospheric input parameters for clouds, aerosols, ozone, water vapor, and surface-reflective properties.

The European program Copernicus provides environmental information to support policymakers, public authorities, and both public and commercial users. Data are provided under the Copernicus data policy, which includes free availability for any use, including commercial use.

The preoperational atmosphere service of Copernicus was provided through the FP7 projects MACC and MACC-II (Monitoring Atmospheric Composition and Climate). On January 1, 2016, the MACC Radiation Service was renamed CAMS Radiation Service once it went operational within CAMS.

The user's guide (Schroedter-Homscheidt et al. 2016) describes the data, methods, and operations used to deliver time series of solar radiation available at ground surface in an easy-toread manner. The HELIOSAT-4 method is based on the decoupling solution proposed by Oumbe et al. (2014) and further described in Qu et al. (2017). The clear-sky McClear model is described in Lefèvre et al. (2013; see Section 4.6.4). Table 4-3 shows an overview of the data used in the CAMS Radiation Service.

Table 4-3. Summary of Data Used in MACC-RAD

\begin{tabular}{|l|l|l|l|}
\hline \multicolumn{1}{|c|}{ Variable } & \multicolumn{1}{|c|}{ Data Sources } & Temporal Resolution & Spatial Resolution \\
\hline Aerosol properties and type & CAMS & 3 hours & $40 \mathrm{~km}$ \\
\hline Cloud properties and type & APOLLO (DLR) & 15 minutes & $3-10 \mathrm{~km}$ \\
\hline Total column content in ozone & CAMS & 3 hours & $40 \mathrm{~km}$ \\
\hline Total column content in water vapor & CAMS & 3 hours & $40 \mathrm{~km}$ \\
\hline Ground albedo & $\begin{array}{l}\text { MODIS (prepared } \\
\text { by Mines } \\
\text { ParisTech) }\end{array}$ & $\begin{array}{l}\text { Climatology of } \\
\text { monthly values }\end{array}$ & $6 \mathrm{~km}$ \\
\hline
\end{tabular}

\section{Perez/Clean Power Research}

The Perez et al. (2002) method (herein referred to as the Perez State University of New York [Perez SUNY] model) evaluates GHI and DNI, based on the concept that the atmospheric transmittance is directly proportional to the TOA planetary albedo (Schmetz 1989). This method is being applied to the GOES satellites and is currently available as the SolarAnywhere product from Clean Power Research. ${ }^{13}$ The concept of using satellite-based measurements of radiance assumes that the visible imagery demonstrates cloud cover for high levels of brightness and lower levels for clearer conditions (e.g., dark ground cover). Readers are referred to Perez et al. (2002) for additional details.

\footnotetext{
${ }^{13}$ See www.cleanpower.com.
} 


\section{Vaisala Solar Data Set}

3Tier (now Vaisala) developed a global solar radiation data set for both GHI and DNI. It follows the method of Perez et al. (2002) using independently developed algorithms. The revised Vaisala algorithms currently use the REST2 clear-sky model and other refinements. This data set is available for global locations at 3-km resolution from $1997 .{ }^{14}$

\section{SolarGIS}

A model for the calculation of global and direct irradiances was implemented for the region covered by the Meteosat, GOES, and Himawari satellites covering land between latitudes $60^{\circ} \mathrm{N}$ and $50^{\circ} \mathrm{S}$. The model philosophy is based on the principles of the Heliosat- 2 calculation scheme (Hammer et al. 2003) and the model by Perez et al. (2002), and it is implemented to operationally process satellite data at a full spatial and temporal resolution.

The model was developed by SolarGIS (Cebecauer and Suri 2010) and includes various enhancements, such as a downscaling capability to take terrain effects and local variability into account.

\section{EnMetSol Model}

The EnMetSol method ${ }^{15}$ is a technique for determining the global radiation at ground level by using data from a geostationary satellite (Beyer et al. 1996; Hammer et al. 2003). It is used in combination with a clear-sky model to evaluate the three usual irradiance components. The key parameter of the method is the cloud index $n$, which is estimated from the satellite measurements and related to the transmissivity of the atmosphere. The method is used for MFG and MSG and GOES data. The EnMetSol method uses the SOLIS model (Mueller et al. 2004) in combination with monthly averages of AOD (Kinne et al. 2005) and water vapor (Kalnay 1996) as input parameters to calculate DNI or spectrally resolved solar irradiance. The DNI for all-sky conditions is calculated with a beam-fraction model (Hammer et al. 2009).

The method uses the clear-sky model of Dumortier (1998; see also Fontoynont et al. 1998) with Remund's (2009) METEONORM HR high-resolution database for the turbidity input. This model is also used to obtain near-real time and forecasts of GTI and GTI as inputs for PV power predictions. The all-sky DHI is calculated with a diffuse fraction model (Lorenz 2007).

\footnotetext{
14 See

http://www.vaisala.com/Vaisala\%20Documents/Scientific\%20papers/3TIER\%20Solar\%20Dataset \%20Methodology \%20and\%20Validation.pdf? ga=1.99089566.1890237981.1394048688.

${ }^{15} \mathrm{See}$ https://www.uni-oldenburg.de/fileadmin/user_upload/physik/ag/ehf/enmet/download/EnMetSol.pdf.
} 


\section{References}

Aler, Ricardo, Inés M Galván, Jose A Ruiz-arias, and Christian A Gueymard. 2017. "Improving the Separation of Direct and Diffuse Solar Radiation Components Using Machine Learning by Gradient Boosting." Solar Energy 150: 558-69. doi:10.1016/j.solener.2017.05.018.

Antonanzas-Torres, F., J. Antonanzas, R. Urraca, M. Alia-Martinez, and F. J. Martinez-de-Pison. 2016. "Impact of Atmospheric Components on Solar Clear-Sky Models at Different Elevation: Case Study Canary Islands." Energy Conversion and Management 109 (2016): 122-9.

Badescu, V., C. A. Gueymard, S. Cheval, C. Oprea, M. Baciu, A. Dumitrescu, F. Iacobescua, I. Milso, and C. Rada. 2012. "Computing Global and Diffuse Solar Hourly Irradiation on ClearSky: Review and Testing of 54 Models." Renewable and Sustainable Energy Reviews 16: 163656.

Bender, G., F. Davidson, F. Eichelberger, and C. A. Gueymard. 2011. "The Road to Bankability: Improving Assessments for More Accurate Financial Planning." In: Proceedings of: Solar 2011 Conf. American Solar Energy Soc., Raleigh, North Carolina.

Besho, K., K. Date, M. Hayashi, A. Ikeda, T. Imai, H. Inoue, Y. Kumagai, T. Miyakawa, H. Murata, T. Ohno, A. Okuyama, R. Oyama, Y. Sasaki, Y. Shimazu, K. Shimoji, and Y. Sumida. 2016. "An Introduction to Himawari-8/9 - Japan's New-Generation Geostationary Meteorological Satellites." Journal of the Meteorological Society of Japan 94: 151-83. doi:10.2151/jmsj.2016-009

Beyer, H., C. Costanzo, and D. Heinemann. 1996. "Modifications of the HELIOSAT Procedure for Irradiance Estimates from Satellite Images." Solar Energy 56(3): 207-21.

Bird, R. E., and R. L. Hulstrom. 1981. A Simplified Clear-Sky Model for Direct and Diffuse Insolation on Horizontal Surfaces. SERI/TR-642-761. Golden, CO: Solar Energy Research Institute.

Blanc, P., B. Gschwind, M. Lefèvre, and L. Wald. 2011. "The HelioClim Project: Surface Solar Irradiance Data for Climate Applications." Remote Sensing 3(12): 343-61. doi: $10.3390 /$ rs 3020343

Blanc, P., Alexandre Boilley, N. Killius, P. Massip, Marion Schroedter-Homscheidt, and L. Wald. 2012. "Methods for Data Fusion." Report of the Project ENDORSE, 7th Framework Programme EU.

Boilley, A., and L. Wald. 2015. "Comparison Between Meteorological Re-Analyses from ERAInterim and MERRA and Measurements of Daily Solar Irradiation at Surface." Renewable Energy 75: 135-43. doi:10.1016/j.renene.2014.09.042

Cano, D., J. M. Monget, M. Albuisson, H. Guillard, N. Regas, and L. Wald. 1986. "A Method for the Determination of the Global Solar Radiation from Meteorological Satellite Data." Solar Energy 37(1): 31-9. 
Cebecauer, T., and M. Suri. 2010. "Accuracy Improvements of Satellite-Derived Solar Resource Based on GEMS Re-Analysis Aerosols.” In: Proceedings of: SolarPACES 2010 Conference, Perpignan, France.

—. 2012. "Correction of Satellite-Derived DNI Time Series Using Locally-Resolved Aerosol Data.” In: Proceedings of: SolarPACES 2012 Conference, Marrakech, Morocco.

Darnell, W. L., W. F. Staylor, S. K. Gupta, and M. Denn. 1988. "Estimation of Surface Insolation Using Sun-Synchronous Satellite Data." Journal of Climate 1: 820-35.

Dedieu, G., P. Y. Deschamps, and Y. H. Kerr. 1987. "Satellite Estimation of Solar Irradiance at the Surface of the Earth and of Surface Albedo Using a Physical Model Applied to Meteosat Data." Journal of Climate and Applied Meteorology 26: 79-87.

Diabate, L., H. Demarcq, N. Michaud-Regas, and L. Wald. 1988. "Estimating Incident Solar Radiation at the Surface From Images of the Earth Transmitted by Geostationary Satellites: The HELIOSAT Project.” International Journal of Solar Energy 5: 261-78.

Diak G. R., and C. Gautier. 1983. "Improvements to a Simple Physical Model for Estimating Insolation from GOES Data.” Journal of Climate and Applied Meteorology 22: 505-8.

Engerer, N. A. 2015. "Minute Resolution Estimates of the Diffuse Fraction of Global Irradiance for Southeastern Australia." Solar Energy 116: 215-37.

Engerer, N. A., and F. P. Mills. 2015. "Validating Nine Clear Sky Radiation Models in Australia.” Solar Energy 120: 9-24.

Erbs, D. G., S. A. Klein, and J. A. Duffie. 1982. Estimation of the Diffuse Radiation Fraction for Hourly, Daily, and Monthly-Average Global Radiation.” Solar Energy 28(4): 293-302. ISSN 0038-092X. http://dx.doi.org/10.1016/0038-092X(82)90302-4.

Espinar, B., L. Ramírez, J. Polo, L. F. Zarzalejo, and L. Wald. 2009. “Analysis of the Influences of Uncertainties in Input Variables on the Outcomes of the Heliosat-2 Method." Solar Energy 83 : 1731-41. doi:10.1016/j.solener.2009.06.010

Gautier, C., G. Diak, and S. Masse. 1980. "A Simple Physical Model to Estimate Incident Solar Radiation at the Surface From GOES Satellite Data." Journal of Applied Meteorology 19: 1,00512.

Gautier, C., and R. Frouin. 1984. "Satellite-Derived Ocean Surface Radiation Fluxes." Proceeding of the Workshop on Advances in Remote Sensing Retrieval Methods, Williamsburg, Virginia.

George, R., S. Wilcox, M. Anderberg, and R. Perez. 2007. "National Solar Radiation Database (NSRDB): 10 Km Gridded Hourly Solar Database.” Solar 2007 Conference Proceedings, July 712, 2007, Cleveland, Ohio. NREL/CP-581-41599. Golden, CO: National Renewable Energy Laboratory. 
Gueymard, C. 1987. "An Anisotropic Solar Irradiance Model for Tilted Surfaces and its Comparison With Selected Engineering Algorithms." Solar Energy 38: 367-86.

doi:10.1016/0038-092X(87)90009-0

Gueymard, C. A. 1993. "Critical Analysis and Performance Assessment of Clear-Sky Irradiance Models Using Theoretical and Measured Data." Solar Energy 51: 121-38.

Gueymard, C. A. 2003a. "Direct Solar Transmittance and Irradiance Predictions with Broadband Models: Part I-Detailed Theoretical Performance Assessment." Solar Energy 74: 355-79.

Gueymard, C. A. 2003b. "Direct Solar Transmittance and Irradiance Predictions with Broadband Models: Part II—Validation with High-Quality Measurements.” Solar Energy 74: 381-95.

Gueymard, C. A. 2004a. "Direct Solar Transmittance and Irradiance Predictions with Broadband Models: Part I-Detailed Theoretical Performance Assessment." Corrigendum Solar Energy 76: 513.

Gueymard, C. A. 2004b. "Direct Solar Transmittance and Irradiance Predictions with Broadband Models: Part II_-Validation with High-Quality Measurements.” Corrigendum Solar Energy 76: 515.

Gueymard, C. A. 2008a. "From Global Horizontal to Global Tilted Irradiance: How Accurate are Solar Energy Engineering Predictions in Practice?" In: Solar 2008 Conference, San Diego, California. American Solar Energy Society.

Gueymard, C. A. 2008b. "REST2: High-Performance Solar Radiation Model for Cloudless-Sky Irradiance, Illuminance and Photosynthetically Active Radiation-Validation with a Benchmark Dataset." Solar Energy 82: 272-85.

Gueymard, C. A. 2011. "Uncertainties in Modeled Direct Irradiance Around the Sahara as Affected by Aerosols: Are Current Datasets of Bankable Quality?" Journal of Solar Energy Engineering 133: 031024.

Gueymard, C. A. 2012. "Clear-Sky Irradiance Predictions for Solar Resource Mapping and Large-Scale Applications: Improved Validation Methodology and Detailed Performance Analysis of 18 Broadband Radiative Models.” Solar Energy 86: 2145-69.

Gueymard, C. A. 2014. "A Review of Validation Methodologies and Statistical Performance Indicators for Modeled Solar Radiation Data: Towards a Better Bankability of Solar Projects." Renewable and Sustainable Energy Reviews 39: 1,024-34.

Gueymard, C. A. 2017. "Cloud and Albedo Enhancement Impacts on Solar Irradiance Using Highfrequency Measurements From Thermopile and Photodiode Radiometers. Part 2: Performance of Separation and Transposition Models for Global Tilted Irradiance." Solar Energy, 153 (2017), pp. 766-779. 
Gueymard, C. A., and D. Myers. 2008. "Validation and Ranking Methodologies for Solar Radiation Models." Badescu, V., ed. Modeling Solar Radiation at the Earth's Surface. Berlin, Germany: Springer.

Gueymard, C. A., and J. A. Ruiz-Arias. 2015. "Validation of Direct Normal Irradiance Predictions Under Arid Conditions: A Review of Radiative Models and Their TurbidityDependent Performance." Renewable and Sustainable Energy Reviews 45: 379-96.

Gueymard, C., and J.A. Ruiz-Arias. 2016. "Extensive Worldwide Validation and Climate Sensitivity Analysis of Direct Irradiance Predictions from 1-Min Global Irradiance." Solar Energy. vol. 128, 1-30. Elsevier Ltd. doi:10.1016/j.solener.2015.10.010

Gueymard, C.A. and D. Thevenard, 2009. "Monthly average clear-sky broadband irradiance database for worldwide solar heat gain and building cooling load calculations". Solar Energy, vol. 83, 1998-2018.

Gueymard, C. A., W. T. Gustafson, G. Bender, A. Etringer, and P. Storck. 2012. "Evaluation of Procedures to Improve Solar Resource Assessments: Optimum Use of Short-Term Data From a Local Weather Station to Correct Bias in Long-Term Satellite-Derived Solar Radiaiton Time Series." Proc. World Renewable Energy Forum, Denver, Colorado, American Solar Energy Society.

Hay, J. E., K. Hanson, and J. Hanson. 1978. "A Satellite-Based Methodology for Determining Solar Irradiance at the Ocean Surface during GATE." Bulletin of the American Meteorological Society 59: 1,549.

Heidinger, A. K. 2003. "Rapid Daytime Estimation of Cloud Properties Over a Large Area from Radiance Distributions." Journal of Atmospheric and Oceanic Technology 20: 1,237-50.

Heidinger, A.K., M.J.Foster, A. Walther, X. Zhao, 2014. "The Pathfinder Atmospheres-Extended (PATMOS-x) AVHRR Climate Data Set." Bulletin of the American Meteorological Society (95); pp. 909-922. http://dx.doi.org/10.1175/BAMS-D-12-00246.1.

Ineichen, P. 2016. "Long Term Helioclim-3 Global, Beam and Diffuse Irradiance Validation." Working paper with appendixes. Available at: https://archive-ouverte.unige.ch/unige:81915.

Joseph, J. H., W. J. Wiscombe, and J. A. Weinman. 1976. "The Delta-Eddington Approximation for Radiative Transfer." Journal of Atmospheric Science 33: 2,452-9.

Justus, C., M. V. Paris, and J. D. Tarpley. 1986. "Satellite-Measured Insolation in the United States, Mexico, and South America." Remote Sensing of Environment 20: 57-83.

Kambezidis, H. D., B. E. Psiloglou, and C. Gueymard. 1994. "Measurements and Models for Total Solar Irradiance on Inclined Surface in Athens, Greece." Solar Energy 53: 177-85. doi:10.1016/0038-092X(94)90479-0

Karlsson, Karl-Göran, Kati Anttila, Jörg Trentmann, Martin Stengel, Jan Fokke Meirink, Abhay Devasthale, Timo Hanschmann, Steffen Kothe, Emmihenna Jääskeläinen, Joseph Sedlar, Nikos 
Benas, Gerd-Jan van Zadelhoff, Cornelia Schlundt, Diana Stein, Stephan Finkensieper, Nina Håkansson, Rainer Hollmann, Petra Fuchs, and Martin Werscheck. 2017. "CLARA-A2: CM SAF cLoud, Albedo and Surface RAdiation Dataset from AVHRR Data - Edition 2." Satellite Application Facility on Climate Monitoring. DOI:10.5676/EUM_SAF_CM/CLARA_AVHRR/V002. http://dx.doi.org/10.5676/EUM_SAF_CM/CLARA_AVHRR/V002

Kerschegens, M., U. Pilz, and E. Raschke. 1978. "A Modified Two-Stream Approximation for Computations of the Solar Radiation in a Cloudy Atmosphere." Tellus 30: 429-35.

Khalil, S. A., and A. M. Shaffie. 2013. "A Comparative Study of Total, Direct and Diffuse Solar Irradiance by Using Different Models on Horizontal and Inclined Surfaces for Cairo, Egypt." Renewable and Sustainable Energy Reviews 27: 853-63. doi:10.1016/j.rser.2013.06.038

Kriebel, K. T., R. W. Saunders, and G. Gesell. 1989. “Optical Properties of Clouds Derived from Fully Cloudy AVHRR Pixels.” Beiträge zur Physik der Atmosphäre 62(3 August): 165-71.

Kriebel K. T., G. Gesell, M. Kästner, H. Mannstein. 2003. "The cloud analysis tool APOLLO: Improvements and Validation." Int. J. Rem. Sens., 24, 2389-2408.

Lefèvre, M., L. Wald, and L. Diabate. 2007. "Using Reduced Datasets ISCCP-B2 From the Meteosat Satellites To Assess Surface Solar Irradiance.” Solar Energy 81: 240-53.

Liu, B. Y. H., and R. C. Jordan. 1960. "The Interrelationship and Characteristic Distribution of Direct, Diffuse and Total Solar Radiation." Solar Energy 4: 1-19. doi:10.1016/0038092X(60)90062-1

Louche, A., G. Notton, P. Poggi, and G. Simonnot. 1991. "Correlations for Direct Normal and Global Horizontal Irradiation on a French Mediterranean Site." Solar Energy 46: 261-6. doi:10.1016/0038-092X(91)90072-5

Loutzenhiser, P. G., H. Manz, C. Felsmann, P. A. Strachan, T. Frank, and G. M. Maxwell. 2007. "Empirical Validation of Models to Compute Solar Irradiance on Inclined Surfaces for Building Energy Simulation." Solar Energy 81: 254-67. doi:10.1016/j.solener.2006.03.009

Marion, W., and S. Wilcox. 1994. Solar Radiation Data Manual for Flat-Plate and Concentrating Collectors. NREL/TP-463-5607. Golden, CO: National Renewable Energy Laboratory.

Möser, W., and E. Raschke. 1983. "Mapping of Global Radiation and Cloudiness from METEOSAT Image Data." Meteorologische Rundschau 36: 33-41.

- 1984. "Incident Solar Radiation over Europe Estimated From METEOSAT Data." Journal of Climate and Applied Meteorology 23: 166-70.

Mueller, R. W., K. F. Dagestad, P. Ineichen, M. Schroedter, S. Cros, D. Dumortier, R. Kuhlemann, J. A. Olseth, G. Piernavieja, C. Reise, L. Wald, and D. Heinnemann. 2004. 
"Rethinking Satellite Based Solar Irradiance Modelling: The SOLIS Clear Sky Module." Remote Sensing of Environment 90(2): 160-74.

Muneer, T., and G. S. Saluja. 1985. "A Brief Review of Models for Computing Solar Radiation on Inclined Surfaces." Energy Conversion and Management 25: 443-58. doi:10.1016/01968904(85)90009-3

Myers, D. R. 2013. Solar Radiation: Practical Modeling for Renewable Energy Applications. Ghassemi, A., ed. New York: Energy and the Environment Series, CRC Press; 182 pp.

NREL. 1992. User's Manual: National Solar Radiation Data Base, 1961-1990. Version 1.0. Golden, CO: National Renewable Energy Laboratory.

NREL. 2007. National Solar Radiation Database Update, 1991-2005. User's Manual. NREL/TP-581-41364. Golden, CO: National Renewable Energy Laboratory.

Nunez, M. 1990. "Solar Energy Statistics for Australian Capital Regions.” Solar Energy 44: 343-54.

Olmo, F., J. Vida, I. Foyo, Y. Castro-Diez, and L. Alados-Arboledas. 1999. "Prediction of Global Irradiance on Inclined Surfaces from Horizontal Global Irradiance." Energy 24: 689-704. doi:10.1016/S0360-5442(99)00025-0

Oumbe, A. 2009. "Exploitation Des Nouvelles Capacités D'observation De La Terre Pour Evaluer Le Rayonnement Solaire Incident Au Sol (Assessment of Solar Surface Radiation Using New Earth Observation Capabilities)." Thèse de Doctorat, MINES ParisTech, Paris, France, 128 pp.

Oumbe, A., Z. Qu, P. Blanc, M. Lefèvre, L. Wald, and S. Cros. 2014. "Decoupling the Effects of Clear Atmosphere and Clouds to Simplify Calculations of the Broadband Solar Irradiance at Ground Level." Geoscientific Model Development Discussions 7(2): 2007-32.

doi:10.5194/gmdd-7-2007-2014

Padovan, A., and D. Del Col. 2010. "Measurement and Modeling of Solar Irradiance Components on Horizontal and Tilted Planes." Solar Energy 84: 2068-84.

doi:10.1016/j.solener.2010.09.009

Page, John K., Michel Albuisson, and Lucien Wald. 2001. "The European Solar Radiation Atlas: A Valuable Digital Tool." Solar Energy 71(1): 81-3.

Perez, R., R. Seals, P. Ineichen, R. Stewart, and D. Menicucci. 1987. "A New Simplified Version of the Perez Diffuse Irradiance Model for Tilted Surfaces." Solar Energy 39: 221-31. doi:10.1016/S0038-092X(87)80031-2

Perez, R., R. Stewart, R. Seals, and T. Guertin. 1988. "The Development and Verification of the Perez Diffuse Radiation Model." Sandia National Lab Technical Report. SAND 88-7030. http://prod.sandia.gov/techlib/access-control.cgi/1988/887030.pdf 
Perez, R., P. Ineichen, R. Seals, J. Michalsky, and R. Stewart. 1990. "Modeling Daylight Availability and Irradiance Components From Direct and Global Irradiance." Solar Energy 44: 271-89. doi:10.1016/0038-092X(90)90055-H

Perez, R., P. Ineichen, K. Moore, M. Kmiecik, C. Chain, R. George, and F. Vignola. 2002. “A New Operational Satellite-to-Irradiance Model.” Solar Energy 73(5): 307-17.

Pinker, R. T., and J. A. Ewing. 1985. "Modeling Surface Solar Radiation: Model Formulation and Validation." Journal of Climate and Applied Meteorology 24: 389-401.

Pinker, R. T., and I. Laszlo 1992. "Modeling of Surface Solar Irradiance for Satellite Applications on a Global Scale." Journal of Applied Meteorology and Climatology 31: 194-211.

Pinker, R. T., R. Frouin, and Z. Li. 1995. “A Review of Satellite Methods to Derive Surface Shortwave Irradiance.” Remote Sensing of Environment 51: 108-24.

Polo, J., L. Martín, and M. Cony. 2012. "Revision of Ground Albedo Estimation in Heliosat Scheme for Deriving Solar Radiation from SEVIRI HRV Channel of Meteosat Satellite." Solar Energy 86: 275-82. doi:10.1016/j.solener.2011.09.030

Polo, J., J. M. Vindel, and L. Martín. 2013. "Angular Dependence of the Albedo Estimated in Models for Solar Radiation Derived From Geostationary Satellites." Solar Energy 93: 256-66. doi:http://dx.doi.org/10.1016/j.solener.2013.04.019

Qu, Z., A. Oumbe, P. Blanc, B. Espinar, G. Gesell, B. Gschwind, L. Klüser, M. Lefèvre, L. Saboret, M. Schroedter-Homscheidt, and L. Wald. 2016. "Fast Radiative Transfer Parameterisation for Assessing the Surface Solar Irradiance: The Heliosat-4 Method. Meteorologische Zeitschrift, 26(1): 33-57.

Remund, J., L. Wald, M. Lefèvre, T. Ranchin, and J. Page. 2003. "Worldwide Linke Turbidity Information." CD-ROM published by International Solar Energy Society, Proceedings of ISES SolarWorld Congress, 16-19 June, Goteborg, Sweden.

Renné, D. S., R. Perez, A. Zelenka, C. Whitlock, and R. DiPasquale. 1999. "Use of Weather and Climate Research Satellites for Estimating Solar Resources." Chapter 5 in Advances in Solar Energy: An Annual Review of Research and Development, Vol. 13. D. Yogi Goswami and K. W. Boer, eds. Boulder, CO: American Solar Energy Society, Boulder.

Rigollier, C., M. Lefèvre, and L. Wald. 2004. "The Method HELIOSAT-2 for Deriving Shortwave Solar Radiation Data From Satellite Images.” Solar Energy 77: 159-69.

Schiffer, R. A., and W. B. Rossow. 1983. "The International Satellite Cloud Climatology Project (ISCCP): The First Project of the World Climate Research Programme." Bulletin of the American Meteorological Society 64: 779-84.

Schmetz, J. 1989. “Towards a Surface Radiation Climatology: Retrieval of Downward Irradiances from Satellites.” Atmospheric Research 23: 287-321. 
Schmetz, J., P. Pili, S. Tjemkes, D. Just, J. Kerkmann, S. Rota, and A. Ratier. 2002. “An Introduction to Meteosat Second Generation (MSG)." Bulletin of the American Meteorological Society 83: 977-92.

Schroedter-Homscheidt, M., C. Hoyer-Klick, N. Killius, M. Lefèvre, L. Wald, E. Wey, and L. Saboret. 2016. "User's Guide to the CAMS Radiation Service.", http://www.sodapro.com/documents/10157/326238/CAMS72_2015SC1_D72.11.3.1_201612_UserGuide_v2.pdf/ ed54f8ec-e19e-4948-af14-d4c2a94083ac

Stuhlmann, R., M. Rieland, and E. Raschke. 1990. "An Improvement of the IGMK Model to Derive Total and Diffuse Solar Radiation at the Surface From Satellite Data." Journal of Applied Meteorology 29: 586-603.

Tapakis, R., S. Michaelides, and A. G. Charalambides. 2016. "Computations of Diffuse Fraction of Global Irradiance: Part 1 - Analytical Modelling.” Solar Energy 139: 711-22.

Tarpley, J. D. 1979. "Estimating Incident Solar Radiation at the Surface from Geostationary Satellite Data." Journal of Applied Meteorology 18: 1,172-81.

Wattan, R., and S. Janjai. 2016. "An Investigation of the Performance of 14 Models for Estimating Hourly Diffuse Irradiation on Inclined Surfaces at Tropical Sites." Renewable Energy 93: 667-74. doi:10.1016/j.renene.2016.02.076

Xie, J., and M. Sengupta. 2016. "Performance Analysis of Transposition Models Simulating Solar Radiation on Inclined Surfaces." In: Proc. European PV Solar Conference and Exhibition (EU PVSEC), Munich, Germany, June 20-24.

Yang, D. 2016. "Solar Radiation on Inclined Surfaces: Corrections and Benchmarks." Solar Energy 136: 288-302.

Zhong, X., and J. Kleissl. 2015. "Clear Sky Irradiances using REST2 and MODIS.” Solar Energy 116: 144-64. 


\title{
5 Solar Resource Data
}

\author{
Aron Habte, ${ }^{1}$ Manajit Sengupta, ${ }^{1}$ Christian Gueymard, ${ }^{2}$ and Thomas Stoffel ${ }^{3}$ \\ 1. National Renewable Energy Laboratory \\ 2. Solar Consulting Services \\ 3. Solar Resource Solutions, $L L C$
}

\subsection{Introduction}

Understanding the long-term spatial and temporal variability of available solar resources is fundamental to any assessment of solar energy potential. Information derived from historical solar resource data can be used to make energy policy decisions, select optimum energy conversion technologies, design systems for specific locations, and operate and maintain installed solar energy conversion systems. Historical solar resource data can be the result of insitu measurement programs, satellite remote-sensing methods, or meteorological model outputs. As described in the previous chapters, each type of data has different information content and applicability.

This chapter summarizes historical solar resource data available around the world. It provides an inventory of representative sources of solar radiation data and a summary of important data characteristics associated with each data source (e.g., period of record, temporal and spatial resolutions, available data elements, and estimated uncertainties).

The National Renewable Energy Laboratory (NREL) and other agencies have made every effort to make data products that are as useful, robust, and representative as possible; however, the responsibility for applying the data correctly resides with the user. A thorough understanding of the data sources, how they are created, and their limitations remains vital to proper application of the resource data to analyses and subsequent decision making. Discussion and examples of the use of several of these data sets for solar energy applications are presented here. Users are encouraged to read the pertinent sections of this chapter before applying solar resource and meteorological data.

Measured solar irradiance data can provide detailed temporal information for a specific site. Because solar radiation measurement stations are challenging to operate and the data collected are not used for routine weather forecasts, they are few in number and have limited data collection records. The largest national measurement network for obtaining hourly solar resource data in the United States was the 39-station National Oceanic and Atmospheric Administration (NOAA) network, which operated from 1977-1980 (see Table 5.1). The current number of highquality stations is about half of that, including NOAA's SURFace RADiation (SURFRAD) network, the University of Oregon network, and some stations from the US Department of Energy's (DOE) Atmospheric Radiation Measurement (ARM) program and NREL. The total number of ground stations measuring solar irradiance in some form and with a wide range of data quality is now more than 3,000 in the United States alone. They are operated by a number of interests producing data for varied applications (including agriculture). For other parts of the world, refer to the Baseline Surface Radiation Network (BSRN) network (http://bsrn.awi.de/) for high-quality data, to each country's meteorological services, and see Table 5.1 (Gueymard and Myers 2008, 2009) for more details on sources of measured or partly measured databases. 
Satellite-based observations and mesoscale meteorological models address the needs for understanding the spatial variability of solar radiation resources throughout a range of distances. Present state-of-the-art models provide estimates for global horizontal irradiance (GHI) and direct normal irradiance (DNI) at spatial resolutions of $10 \mathrm{~km}$ or less for the United States and other parts of the world (see, e.g., SolarGIS in Table 5.1 ). The rapidly growing needs for more accurate solar resource information throughout shorter temporal and smaller spatial scales require the user to fully appreciate the characteristics of all available data, especially those from historical sources.

\subsection{Solar Resource Data Characteristics}

Characterizing the available solar resources for solar energy applications is important for all aspects of realizing the full potential of this utility-scale energy source. Energy policy decisions, engineering designs, and system deployment considerations require an accurate understanding of the relevant historical solar resource data, the ability to assess the accuracy of current solar measurement and modeling techniques, and forecasts of the levels of solar irradiance for various temporal and spatial scales.

Measured solar irradiance data can provide information about the temporal variability at a specific site. Practical radiometer designs were developed in the early 1900s to determine the sun's energy output based on high-altitude measurements of DNI made with pyrheliometers (Hulstrom 1989). To address the needs of agriculture for monitoring such quantities as evapotranspiration, the U.S. Weather Bureau (now National Weather Service) deployed a national radiometer network in the 1950s to collect GHI. Since then, radiometer design and data acquisition system performance has advanced considerably.

The earliest records of solar flux measurements were based on thermopile-type pyranometer signals recorded and stored on analog strip charts to determine daily amounts of solar flux on a horizontal surface. Today, 1-minute (or shorter) digital recordings are available from fastresponse silicon photodiodes and improved thermopile-type pyranometers and pyrheliometers that are deployed in regional measurement networks to provide solar energy resource data for a variety of applications.

Historically, there have been four radiometer calibration reference scales: Ångström Scale (ÅS; created in 1905), Smithsonian Scale (SS; created in 1913), International Pyrheliometric Scale (IPS; created in 1956), and the World Radiometric Reference (WRR; 1979). The relative differences among these scales can introduce a data bias on the order of $2 \%$. The user should be aware of this potential bias in data measured before 1979. A correction is necessary to harmonize older data sets to the current scale, according to:

- $\quad \mathrm{WRR}=1.026(\AA \mathrm{S} 1905)$

- $\quad \mathrm{WRR}=0.977(\mathrm{SS} 1913)$

- $\quad$ WRR $=1.022($ IPS 1956).

Modeled solar resource data derived from available surface meteorological observations and satellite measurements provide estimates of solar resource potential for locations lacking actual measurements. These modeling methods address the needs for improved spatial resolution of the 
resource data. In the United States, the first national effort to model solar resources in the 1970s advanced our understanding of solar radiation distributions based on the then-available historical measurements at 26 locations to an additional 222 meteorological observing stations with detailed records of hourly cloud amounts and other relevant data (see Table 5.1 for SOLMET/ERSATZ). Today, satellite-based observations of clouds are used to model sub-hourly surface solar fluxes with a 4-km spatial resolution over North America and a part of South America (see Sengupta et al. 2014). Similar efforts are conducted over other parts of the world.

\subsection{Long-Term and TMY Data Sets}

Understanding the time frame, or period of record, associated with solar resource and other meteorological data is important for conducting useful analyses. These weather-driven data have fluctuations that can range from seconds to years and longer. Long-term data can be representative of the climate if the period of record is at least 30 years. By convention, the meteorological community has deemed that according to the 1933 International Meteorological Conference in Warsaw, a 30-year interval is sufficient to reflect longer-term climatic trends and filter the short-term inter-annual fluctuations and anomalies. Climate "normals" are recomputed each decade to address temperature, pressure, precipitation, and other surface meteorological variables. Note that the term normal is not equivalent to "average" and has a specific meaning in the meteorological and climatological community. Namely, normal refers to the 30 -year average of an observed parameter that is updated every 10 years (Arguez and Vose 2011). Thus, the averaging period shifts every 10 years.

Often plant project developers require "typical" meteorological information for a potential plant site for prefeasibility studies. A typical meteorological year (TMY) data set provides designers and other users with a small sized annual data set that holds 8,760 hourly meteorological values that typify conditions at a specific location throughout a longer period, such as the 30 -year climatic normal. Different types of TMYs exist. Twelve typical meteorological months (TMMs) selected on the basis of their similarity of individual cumulative frequency distributions for selected data elements comprise the TMY data set. The longer-term distributions are determined for that month using data from the full period of record. The TMMs are then concatenated, essentially without modification, to form a single year with a serially complete data record. The resulting TMY data set contains measured and/or modeled time series solar radiation and surface meteorological data, although some hourly records may contain filled or interpolated data for periods when original observations are missing from the data archive.

TMY data sets are widely used by building designers and others for rough modeling of renewable energy conversion systems. Although not designed to provide meteorological extremes, TMY data have natural diurnal and seasonal variations and represent a year of typical climatic conditions for a location. A TMY data set should not be used to predict weather or solar resources for a particular period of time, nor is it an appropriate basis for evaluating real-time energy production or the detailed power plant design. Rather, a TMY data set represents conditions judged to be typical throughout a long period, such as 30 years. Because it represents typical rather than extreme conditions, it is not suited for designing systems and their components to meet the worst-case weather conditions that could occur at a location. Additionally, a TMY is not well suited to assess any probability of energy yield exceedances 
because the natural variability is most likely not fully described with its correct statistical distribution.

\subsubsection{Key Considerations}

Applying solar and meteorological data from different sources requires attention to these key considerations:

- Period of record. Influenced by many factors, solar resource data vary yearly, seasonally, monthly, weekly, daily, and on timescales down to a few seconds. Thus, climate normals are based on 30 years of meteorological data. But the 30 -year averaging period is updated (shifted) every 10 years. The normal for one period will not likely be the same as a normal for previous or successive periods. Another popular approach is to determine a TMY data set from a statistical analysis of multiyear data to derive a single year of data that are representative of a longer-term record. Comparative analyses must account for any natural differences that result from the periods when the data were acquired.

- Temporal resolution. Solar resource data can range from annually averaged daily irradiation, typically used for mapping resource distributions, to 1-second samples of irradiance for operational time-series analyses. Other considerations depend on the data type.

- Units. The unit of irradiance is $\mathrm{W} / \mathrm{m}^{2}$. The most common unit of irradiation, or integrated power, is $\mathrm{kWh} / \mathrm{m}^{2}$. The actual SI unit for irradiation, $\mathrm{J} / \mathrm{m}^{2}$, is rarely used anymore. The conversion is $1 \mathrm{kWh} / \mathrm{m}^{2}=3.6 \mathrm{MJ} / \mathrm{m}^{2}$. Note that daily-average irradiation data produced by or for climatologists are most often incorrectly reported with a unit of $\mathrm{W} / \mathrm{m}^{2}$. What a daily irradiation of $1 \mathrm{~W} / \mathrm{m}^{2}$ actually means here is an average irradiance of $1 \mathrm{~W} / \mathrm{m}^{2}$ over 24 hours, or $24 \mathrm{Wh} / \mathrm{m}^{2}$. This can create confusion, unfortunately. A daily irradiation should be expressed in $\mathrm{kWh} / \mathrm{m}^{2}$, not $\mathrm{kWh} / \mathrm{m}^{2} /$ day, even though this is a frequent mistake.

- Spatial coverage. The area represented by the data can range from a single station to a sample geographic region to a global (world) perspective.

- Spatial resolution. Ground-based measurements are site specific. Current satellite remote-sensing estimates can be representative of $10-\mathrm{km}$ by $10-\mathrm{km}$ or smaller areas.

- Data elements and sources of the data. The usefulness of solar resource data may depend on the available data elements (e.g., DNI, GHI etc) and whether the data were measured, modeled, or produced from a combination of measurement and models

- Time stamp. There are three possible time references: Local Apparent Time (LAT, also known as Apparent Solar Time), Local Standard Time (LST), and Universal Time (UT). The former is rarely used anymore. Global databases tend to use UT, but there is no general rule. Moreover, for comparative purposes, it is also important to consider what each time stamp specifically refers to. Depending on database, it can be the start, the midpoint, or the end of the time period (for sub-daily data).

- Availability. Data are distributed in the public domain, for purchase or by license. 
Table 5-1. Inventory of Solar Resource Data Sources, Presented in Alphabetical Order

\begin{tabular}{|c|c|c|c|c|c|c|}
\hline Database & $\begin{array}{l}\text { Period of } \\
\text { record }\end{array}$ & $\begin{array}{l}\text { Temporal } \\
\text { resolution }\end{array}$ & Spatial coverage & $\begin{array}{l}\text { Spatial } \\
\text { resolution }\end{array}$ & Data elements and sources & Availability \\
\hline $\begin{array}{l}\text { 3TIER (VAISALA) } \\
\text { Solar Time Series }\end{array}$ & $\begin{array}{l}\text { January } \\
1997- \\
\text { Present }\end{array}$ & $\begin{array}{l}\text { \%30-minute } \\
\text { instantaneous } \\
\text { and 1-hour } \\
\text { averages }\end{array}$ & Global & $2 \operatorname{arc}-\min (\sim 3 \mathrm{~km})$ & $\begin{array}{c}\mathrm{GHI}, \mathrm{DNI} \text {, and } \mathrm{DHI} \text { from model estimates } \\
\text { based on satellite remote-sensing input } \\
\text { data }\end{array}$ & $\begin{array}{c}\text { 3-TIER: } \\
\text { http://www.3tier.com/products/ca } \\
\underline{\text { talog/?tab=solar-catalog }}\end{array}$ \\
\hline $\begin{array}{l}\text { Atmospheric } \\
\text { Radiation } \\
\text { Measurement } \\
\text { Program (ARM) }\end{array}$ & $\begin{array}{c}1997- \\
\text { present }\end{array}$ & $\begin{array}{l}\text { 20-second } \\
\text { instantaneous } \\
\text { samples and 1- } \\
\text { minute } \\
\text { averages of 2- } \\
\text { second scans }\end{array}$ & $\begin{array}{l}\text { Southern Great } \\
\text { Plains, North Slope of } \\
\text { Alaska, and tropical } \\
\text { western Pacific }\end{array}$ & $\begin{array}{l}23 \text { stations } \\
\text { (southern Great } \\
\text { Plains), } 2 \text { stations } \\
\text { (North Slope of } \\
\text { Alaska), and } 2 \\
\text { stations (tropical } \\
\text { western Pacific) }\end{array}$ & $\begin{array}{l}\text { GHI, DNI, DHI, DIR, UIR, and upwelling } \\
\text { (reflected) shortwave irradiance. } \\
\text { Measurements from the Eppley } \\
\text { Laboratory, Inc., Model PSP (GHI, DHI, } \\
\text { and upwelling shortwave irradiance), } \\
\text { Model 8-48 (DHI after 2000), Model NIP } \\
\text { (DNI), and Model PIR (DIR and UIR) }\end{array}$ & $\begin{array}{c}\text { DOE, Atmospheric Radiation } \\
\text { Measurement Climate Research } \\
\text { Facility: http://www.arm.gov. } \\
\text { Data sets are labeled SIRS, } \\
\text { SKYRAD, and GNDRAD. SIRS } \\
\text { data are also submitted to the } \\
\text { BSRN archives: } \\
\text { http://www.bsrn.awi.de/ }\end{array}$ \\
\hline $\begin{array}{l}\text { Baseline Surface } \\
\text { Radiation Network } \\
\text { (BSRN) }\end{array}$ & $\begin{array}{l}1992- \\
\text { present }\end{array}$ & 1-minute & Global & $\begin{array}{l}52 \text { measurement } \\
\text { stations in } \\
\text { operation; } 7 \\
\text { additional stations } \\
\text { (now closed) with } \\
\text { historical data }\end{array}$ & $\begin{array}{l}\text { The number and type of measurements } \\
\text { vary with station. Basic radiation } \\
\text { measurements include GHI, DNI, DHI, } \\
\text { downwelling infrared irradiance, upwelling } \\
\text { infrared irradiance, and upwelling } \\
\text { (reflected) shortwave irradiance. } \\
\text { Measurements are from radiometers of } \\
\text { various manufacturers. Synoptic } \\
\text { meteorological observations, upper air } \\
\text { measurements, and numerous expanded } \\
\text { and supporting measurements are } \\
\text { available. }\end{array}$ & $\begin{array}{l}\text { The WRMC provides web-based } \\
\text { and FTP data access: } \\
\text { http://www.bsrn.awi.de/en/home/ }\end{array}$ \\
\hline $\begin{array}{l}\text { CAMS McClear } \\
\text { Service }\end{array}$ & $\begin{array}{l}2004- \\
\text { present }\end{array}$ & $\begin{array}{c}1 \text { minute, } 15 \\
\text { minutes, } 1 \\
\text { hour, } 1 \text { day, } 1 \\
\text { month, }\end{array}$ & Global & $\begin{array}{l}\text { Various input data } \\
\text { sources with } \\
\text { different spatial } \\
\text { resolutions are } \\
\text { interpolated to the } \\
\text { location of interest }\end{array}$ & $\begin{array}{l}\text { clear sky global, direct, direct normal, } \\
\text { diffuse irradiances, all inputs used to } \\
\text { describe on aerosols, clouds, water vapor, } \\
\text { trace gases, surface reflectivity } \\
\text { parameters }\end{array}$ & $\frac{\underline{\text { http://solar.atmosphere.copernic }}}{\text { us.eu/cams-mcclear }}$ \\
\hline
\end{tabular}




\begin{tabular}{|c|c|c|c|c|c|c|}
\hline Database & $\begin{array}{l}\text { Period of } \\
\text { record }\end{array}$ & $\begin{array}{l}\text { Temporal } \\
\text { resolution }\end{array}$ & Spatial coverage & $\begin{array}{l}\text { Spatial } \\
\text { resolution }\end{array}$ & Data elements and sources & Availability \\
\hline $\begin{array}{l}\text { CAMS Radiation } \\
\text { Service }\end{array}$ & $\begin{array}{c}2004- \\
\text { present }\end{array}$ & $\begin{array}{l}1 \text { minute, } 15 \\
\text { minutes, } 1 \\
\text { hour, } 1 \text { day, } 1 \\
\text { month, }\end{array}$ & $\begin{array}{l}\text { Europe/Africa/Middle } \\
\text { East/Atlantic Ocean }\end{array}$ & $\begin{array}{l}\text { Various input data } \\
\text { sources with } \\
\text { different spatial } \\
\text { resolutions are } \\
\text { interpolated to the } \\
\text { location of interest }\end{array}$ & $\begin{array}{l}\text { all-sky GHI, DNI, DIR, DHI, and } \\
\text { corresponding clear-sky irradiances, all } \\
\text { inputs used to describe on aerosols, } \\
\text { clouds, water vapor, trace gases, surface } \\
\text { reflectivity parameters }\end{array}$ & $\frac{\text { http://solar.atmosphere.copernic }}{\underline{\text { us.eu/cams-radiation-service }}}$ \\
\hline CERES SYN1deg & 2000-2016 & 3-hourly & Global & $1^{\circ} \times 1^{\circ}$ & $\begin{array}{c}\mathrm{GHI}, \mathrm{DHI} \text {, and DNI based on physical } \\
\text { modeling and satellite-based cloud } \\
\text { observations }\end{array}$ & $\begin{array}{l}\frac{\text { https://ceres- }}{\text { tool.larc.nasa.gov/ord- }} \\
\text { tool/isp/SYN1degSelection.jsp }\end{array}$ \\
\hline $\begin{array}{l}\text { Clean Power } \\
\text { Research- } \\
\text { SolarAnywhere }\end{array}$ & $\begin{array}{c}1998- \\
\text { present }\end{array}$ & Hourly & $\begin{array}{l}\text { Continental United } \\
\text { States and Hawaii }\end{array}$ & $10 \mathrm{~km}$ & $\begin{array}{l}\text { GHI, DNI, wind speed, and ambient air } \\
\text { temperature }\end{array}$ & $\begin{array}{c}\text { Clean Power Research: } \\
\underline{\text { http://www.cleanpower.com/Sola }} \\
\underline{\text { rAnywhere }}\end{array}$ \\
\hline $\begin{array}{l}\text { CM SAF cLoud, } \\
\text { Albedo and } \\
\text { surface RAdiation } \\
\text { data set from } \\
\text { AVHRR data - } \\
\text { Edition } 2 \text { (CLARA- } \\
\text { A2) }\end{array}$ & 1982-2015 & $\begin{array}{l}\text { Monthly } \\
\text { averages }\end{array}$ & Global & $0.25^{\circ} \times 0.25^{\circ}$ & $\begin{array}{l}\text { Cloud properties, surface albedo, and } \\
\text { surface radiation parameters derived from } \\
\text { the AVHRR sensor onboard polar-orbiting } \\
\text { NOAA and METOP satellites. }\end{array}$ & $\begin{array}{c}\text { CM-SAF: } \\
\text { http://www.cmsaf.eu/ }\end{array}$ \\
\hline $\begin{array}{l}\text { CM-SAF } \\
\text { Operational } \\
\text { products }\end{array}$ & $\begin{array}{l}2010- \\
\text { present }\end{array}$ & Daily & Europe, Africa & $15 \mathrm{~km}$ & $\begin{array}{c}\text { Based on MSG, provides } \mathrm{GHI} \text { and the } \\
\text { horizontal projection of } \mathrm{DNI}\end{array}$ & https://wui.cmsaf.eu \\
\hline
\end{tabular}




\begin{tabular}{|c|c|c|c|c|c|c|}
\hline Database & $\begin{array}{l}\text { Period of } \\
\text { record }\end{array}$ & $\begin{array}{l}\text { Temporal } \\
\text { resolution }\end{array}$ & Spatial coverage & $\begin{array}{l}\text { Spatial } \\
\text { resolution }\end{array}$ & Data elements and sources & Availability \\
\hline DAYMET & $1980-1997$ & Daily & $\begin{array}{l}\text { Continental United } \\
\text { States }\end{array}$ & $1 \mathrm{~km}$ & $\begin{array}{l}\text { GHI, air temperature (minimum and } \\
\text { maximum), relative humidity, and } \\
\text { precipitation }\end{array}$ & http://daymet.ornl.gov/. \\
\hline DLR ISIS & $\begin{array}{l}\text { July 1983- } \\
\text { December } \\
2004\end{array}$ & 3-hour & Global & $280 \mathrm{~km}$ by $280 \mathrm{~km}$ & $\begin{array}{l}\mathrm{DNI} \text { and GHI from a radiative transfer } \\
\text { model using cloud and aerosol inputs }\end{array}$ & http://www.pa.op.dlr.de/ISIS/ \\
\hline EnMetSol & $\begin{array}{c}\text { 1995- } \\
\text { present } \\
\text { (1995-2004 } \\
\text { based on } \\
\text { Meteosat } \\
\text { First } \\
\text { Generation } \\
\text {-MFG; } \\
\text { 2005- } \\
\text { present } \\
\text { based on } \\
\text { Meteosat } \\
\text { Second } \\
\text { Generation } \\
\text {-MSG); }\end{array}$ & $\begin{array}{l}\text { 30-minute for } \\
\text { MFG; } 15- \\
\text { minute for MSG }\end{array}$ & $\begin{array}{l}\text { Continental Europe, } \\
\text { Canary Islands, } \\
\text { Turkey, and Israel }\end{array}$ & $\begin{array}{l}2.5 \mathrm{~km} \text { for MFG; } 1 \\
\mathrm{~km} \text { for } \mathrm{MSG}\end{array}$ & $\mathrm{GHI}, \mathrm{DHI}$, and DNI & $\begin{array}{c}\text { University of Oldenburg: } \\
\text { http://www.energiemeteorologie. } \\
\underline{\text { de }}\end{array}$ \\
\hline ESRA & $1981-1990$ & $\begin{array}{l}\text { Monthly and } \\
\text { annual average } \\
\text { daily totals } \\
\left(\mathrm{kWh} / \mathrm{m}^{2}\right)\end{array}$ & Europe & $10 \mathrm{~km}$ & $\begin{array}{c}\text { GHI, DNI, and DHI, sunshine duration, air } \\
\text { temperatures, precipitation, water vapor } \\
\text { pressure, and air pressure in a number of } \\
\text { stations }\end{array}$ & $\begin{array}{l}\text { Les Presses Mines Paris Tech: } \\
\text { http://www.mines- } \\
\text { paristech.fr/Ecole/Culture- } \\
\frac{\text { scientifique/Presses-des- }}{\text { mines/\#54. See also }} \\
\frac{\text { http://www.soda- }}{\text { is.com/eng/index.html }}\end{array}$ \\
\hline $\begin{array}{l}\text { Green Power } \\
\text { Labs: } \\
\text { SolarSatData } \\
\text { TM }\end{array}$ & $\begin{array}{c}1995- \\
\text { present } \\
\text { (Americas) } \\
\\
2000- \\
\text { present } \\
\text { (Europe) } \\
\\
2005- \\
\text { present } \\
\text { (Asia, } \\
\text { Australia) }\end{array}$ & 30-min & $\begin{array}{l}\text { Americas, Asia, } \\
\text { Australia, Europe }\end{array}$ & $1-4 \mathrm{~km}$ & $\begin{array}{l}\text { GHI, DNI, DHI, GTI, temperature, } \\
\text { pressure, wind speed, ozone, water vapor, } \\
\text { total cloud fraction } \\
\text { Irradiance time series for P10, P50, P90, } \\
\text { P95 exceedance probabilities }\end{array}$ & $\begin{array}{c}\text { Green Power Labs: } \\
\text { https://greenpowerlabs.com/ }\end{array}$ \\
\hline
\end{tabular}




\begin{tabular}{|c|c|c|c|c|c|c|}
\hline Database & $\begin{array}{l}\text { Period of } \\
\text { record }\end{array}$ & $\begin{array}{l}\text { Temporal } \\
\text { resolution }\end{array}$ & Spatial coverage & $\begin{array}{l}\text { Spatial } \\
\text { resolution }\end{array}$ & Data elements and sources & Availability \\
\hline HelioClim v2-v5 & $\begin{array}{l}2004- \\
\text { present }\end{array}$ & 15-minute & Europe and Africa & $5 \mathrm{~km}$ & $\begin{array}{l}\text { Hourly and daily GHI from satellite remote- } \\
\text { sensing mode }\end{array}$ & $\begin{array}{l}\text { Mines ParisTech Armines } \\
\text { Center for Energy and } \\
\text { Processes. http://www.soda- } \\
\text { pro.com/. See also: www.soda- } \\
\text { is.com/eng/index.html }\end{array}$ \\
\hline $\begin{array}{l}\text { Historically Black } \\
\text { Colleges and } \\
\text { Universities Solar } \\
\text { Measurement } \\
\text { Network }\end{array}$ & 1985-1996 & 5-minute & $\begin{array}{c}\text { Southeastern United } \\
\text { States: Daytona } \\
\text { Beach, Florida; } \\
\text { Savannah, Georgia; } \\
\text { Itta Bena, Mississippi; } \\
\text { Elizabeth City, North } \\
\text { Carolina; } \\
\text { Orangeburg, South } \\
\text { Carolina; and } \\
\text { Bluefield, West } \\
\text { Virginia }\end{array}$ & $\begin{array}{l}\text { Six measurement } \\
\text { stations }\end{array}$ & $\begin{array}{l}\text { GHI, DNI (at three stations), DHI } \\
\text { (shadowband) from measurements by the } \\
\text { Eppley Laboratory, Inc. Model PSP } \\
\text { pyranometers and Model NIP } \\
\text { pyrheliometers mounted in automatic solar } \\
\text { trackers (LI-COR Model 2020). }\end{array}$ & $\begin{array}{c}\text { NREL RReDC: } \\
\text { http://rredc.nrel.gov/solar/old da } \\
\text { ta/hbcu/ (includes quality- } \\
\text { assessed monthly data files, } \\
\text { monthly summary reports, and } \\
\text { monthly irradiance plots) }\end{array}$ \\
\hline $\begin{array}{l}\text { International } \\
\text { Daylight } \\
\text { Measurement } \\
\text { Program (IDMP) }\end{array}$ & $1991-1994$ & Variable & $\begin{array}{c}\text { Australia, Canada, } \\
\text { China, France, } \\
\text { Germany, Greece, } \\
\text { India, Indonesia, } \\
\text { Israel, Japan, Korea, } \\
\text { The Netherlands, } \\
\text { New Zealand, } \\
\text { Portugal, Russia, } \\
\text { Singapore, Slovakia, } \\
\text { Spain, Sweden, } \\
\text { Switzerland, United } \\
\text { Kingdom, and the } \\
\text { United States } \\
\end{array}$ & $\begin{array}{l}43 \text { measurement } \\
\text { stations }\end{array}$ & $\begin{array}{c}\text { GHI, DNI, DHI, zenith luminance, } \\
\text { illuminance (including vertical surfaces), } \\
\text { air temperature, relative humidity (or dew } \\
\text { point), wind speed and direction, bright } \\
\text { sunshine duration, sky imagers, and sky } \\
\text { scanners }\end{array}$ & $\begin{array}{l}\text { École Nationale des Travaux } \\
\text { Publics: http://idmp.entpe.fr/ }\end{array}$ \\
\hline IrSoLaV & $\begin{array}{c}\text { 1994- } \\
\text { present } \\
\text { (Africa, } \\
\text { Europe) } \\
\\
2000- \\
\text { present } \\
\text { (Americas, } \\
\text { western } \\
\text { Asia) }\end{array}$ & $\begin{array}{l}\text { 15-min (except } \\
\text { Americas), 30- } \\
\text { min, hourly, } \\
\text { daily, monthly }\end{array}$ & $\begin{array}{c}\text { Europe, Africa, } \\
\text { Americas, western } \\
\text { Asia }\end{array}$ & $\approx 3 \mathrm{~km}$ & $\begin{array}{l}\mathrm{GHI}, \mathrm{DNI}, \mathrm{DHI}, \mathrm{GTI} \text {, temperature, } \\
\text { pressure, wind, precipitation }\end{array}$ & $\begin{array}{c}\text { IrSoLaV } \\
\text { http:///irsolav.com/ }\end{array}$ \\
\hline
\end{tabular}




\begin{tabular}{|c|c|c|c|c|c|c|}
\hline Database & $\begin{array}{l}\text { Period of } \\
\text { record }\end{array}$ & $\begin{array}{l}\text { Temporal } \\
\text { resolution }\end{array}$ & Spatial coverage & $\begin{array}{l}\text { Spatial } \\
\text { resolution }\end{array}$ & Data elements and sources & Availability \\
\hline $\begin{array}{l}\text { Management and } \\
\text { Exploitation of } \\
\text { Solar Resource } \\
\text { Knowledge } \\
\text { (MESoR) }\end{array}$ & $\begin{array}{l}\text { 1991-2005: } \\
\text { Europe and } \\
\text { Africa; } \\
\text { 1999-2006: } \\
\text { Asia }\end{array}$ & Hourly & $\begin{array}{l}\text { Europe, Western } \\
\text { Asia, Africa, parts of } \\
\text { Australia, South } \\
\text { America }\end{array}$ & $2.5 \mathrm{~km}$ & $\begin{array}{l}\mathrm{GHI}, \mathrm{DNI}, \mathrm{DHI} \text { from ground measurements } \\
\text { and modeling results }\end{array}$ & DLR: www.mesor.org/ \\
\hline METEONORM & $1991-2010$ & $\begin{array}{l}\text { 1-minute and } \\
\text { hourly modeled } \\
\text { data }\end{array}$ & Global & $\begin{array}{l}\text { Data from } 8,351 \\
\text { meteorological } \\
\text { stations are } \\
\text { interpolated to } \\
\text { establish weather } \\
\text { data at any } \\
\text { specified point. } \\
\text { Satellite: } 2 \mathrm{~km} \text { for } \\
\text { Europe, } 8 \mathrm{~km} \text { for } \\
\text { rest of the world }\end{array}$ & $\begin{array}{l}\text { Measured: monthly means of GHI, } \\
\text { temperature, humidity, precipitation, wind } \\
\text { speed and direction, and bright sunshine } \\
\text { duration. Modeled: } 1 \text {-minute and hourly } \\
\text { typical years radiation parameters (GHI, } \\
\text { DNI, DHI, GTI, downwelling infrared, } \\
\text { luminance, and ultraviolet-A and -B), } \\
\text { precipitation, and humidity parameters } \\
\text { (dew point, relative humidity, mixing ratio, } \\
\text { psychrometric temperature) }\end{array}$ & $\begin{array}{c}\text { METEOTEST, Bern, } \\
\text { Switzerland: } \\
\text { http://www.meteonorm.com/ }\end{array}$ \\
\hline $\begin{array}{l}\text { NASA Modern-Era } \\
\text { Retrospective } \\
\text { analysis for } \\
\text { Research and } \\
\text { Applications, } \\
\text { Version 2 } \\
\text { (MERRA-2) }\end{array}$ & $\begin{array}{c}1980- \\
\text { present }\end{array}$ & Hourly & Global & $0.5^{\circ} \times 0.625^{\circ}$ & $\begin{array}{l}\text { GHI, detailed information on clouds, } \\
\text { atmospheric constituents (aerosols, ...), } \\
\text { weather variables (temperature, wind...), } \\
\text { and surface albedo }\end{array}$ & $\frac{\text { https://gmao.gsfc.nasa.gov/rean }}{\text { alysis/MERRA-2/data access/ }}$ \\
\hline $\begin{array}{l}\text { NASA Surface } \\
\text { Meteorology and } \\
\text { Solar Energy }\end{array}$ & $\begin{array}{l}\text { July } 1983- \\
\text { June } 2005\end{array}$ & $\begin{array}{l}\text { Monthly and } \\
\text { annual average } \\
\text { daily totals } \\
\left(\mathrm{kWh} / \mathrm{m}^{2}\right)\end{array}$ & Global & $1 \times 1^{\circ}$ & $\begin{array}{l}\text { GHI, DNI, and DHI from a satellite remote- } \\
\text { sensing model. Also available: estimates } \\
\text { of clear-sky GHI, DNI, and DHI and tilted } \\
\text { surface irradiance, temperature, pressure, } \\
\text { humidity, precipitation, and wind speed }\end{array}$ & $\begin{array}{c}\text { NASA SSE website: } \\
\text { http://eosweb.larc.nasa.gov/sse/ }\end{array}$ \\
\hline $\begin{array}{l}\text { National Center for } \\
\text { Environmental } \\
\text { Protection/National } \\
\text { Center for } \\
\text { Atmospheric } \\
\text { Research Global } \\
\text { Reanalysis } \\
\text { Products (NCEP) } \\
\end{array}$ & 1948-2009 & 6-hour $\left(\mathrm{W} / \mathrm{m}^{2}\right)$ & Global & $2.5^{\circ}$ (nominal) & $\begin{array}{l}\mathrm{GHI} \text { and more than } 80 \text { variables, including } \\
\text { geopotential height, temperature, relative } \\
\text { humidity, and } U \text { and } \mathrm{V} \text { wind components, } \\
\text { in several coordinate systems, such as a } \\
\text { 17-pressure-level stack on } 2.5 \times 2.5^{\circ} \text { grids, } \\
28 \text { sigma-level stacks on } 192 \text { by } 94 \\
\text { Gaussian grids, and } 11 \text { isentropic-level } \\
\text { stacks on a } 2.5 \times 2.5^{\circ} \text { grid } \\
\end{array}$ & $\begin{array}{c}\text { University Center for } \\
\text { Atmospheric Research, } \\
\text { Computational and Information } \\
\text { Systems Laboratory Research } \\
\text { Data Archive: } \\
\text { http://rda.ucar.edu/datasets/ds0 } \\
\underline{90.0 /}\end{array}$ \\
\hline
\end{tabular}




\begin{tabular}{|c|c|c|c|c|c|c|}
\hline Database & $\begin{array}{l}\text { Period of } \\
\text { record }\end{array}$ & $\begin{array}{l}\text { Temporal } \\
\text { resolution }\end{array}$ & Spatial coverage & $\begin{array}{l}\text { Spatial } \\
\text { resolution }\end{array}$ & Data elements and sources & Availability \\
\hline NOAA Network & $1977-1980$ & Hourly & $\begin{array}{l}\text { United States and } \\
\text { territories }\end{array}$ & $\begin{array}{l}39 \text { measurement } \\
\text { stations }\end{array}$ & $\begin{array}{c}\text { GHI, DNI, DHI (7 stations), air } \\
\text { temperature, relative humidity, cloud } \\
\text { amounts, barometric pressure, wind speed } \\
\text { and direction at } 10 \mathrm{~m} \text {, precipitation, snow } \\
\text { cover, and weather codes measured } \\
\text { according to standard NWS operating } \\
\text { procedures. }\end{array}$ & $\begin{array}{l}\text { NCDC; National Environmental, } \\
\text { Satellite, Data, and Information } \\
\text { Service; NOAA; U.S. } \\
\text { Department of Commerce: } \\
\text { http://www.ncdc.noaa.gov/ }\end{array}$ \\
\hline $\begin{array}{l}\text { NOAA Network - } \\
\text { Integrated Surface } \\
\text { Irradiance Study } \\
\text { (ISIS) }\end{array}$ & 1995-2006 & 15-minute & $\begin{array}{c}\text { Continental United } \\
\text { States }\end{array}$ & $\begin{array}{c}\text { Nine stations: } \\
\text { Albuquerque, } \\
\text { New Mexico; } \\
\text { Bismarck, North } \\
\text { Dakota; Desert } \\
\text { Rock, Nevada; } \\
\text { Hanford, } \\
\text { California; } \\
\text { Madison, } \\
\text { Wisconsin; Oak } \\
\text { Ridge, } \\
\text { Tennessee; } \\
\text { Seattle, } \\
\text { Washington; Salt } \\
\text { Lake City, Utah; } \\
\text { Sterling, Virginia; } \\
\text { and Tallahassee, } \\
\text { Florida. } \\
\end{array}$ & GHI, DNI, DHI, and Global UVB & $\begin{array}{l}\text { NOAA, Earth Systems Research } \\
\text { Laboratory, Global Monitoring } \\
\text { Division, Boulder, Colorado: } \\
\frac{\text { ftp://ftp.srrb.noaa.gov/pub/data/i }}{\underline{\text { sis/ }}}\end{array}$ \\
\hline $\begin{array}{c}\text { NOAA Network - } \\
\text { Surface Radiation } \\
\text { Network } \\
\text { (SURFRAD) }\end{array}$ & $\begin{array}{c}\text { 1993- } \\
\text { present }\end{array}$ & $\begin{array}{c}\text { Data are } \\
\text { reported as 3- } \\
\text { minute } \\
\text { averages of 1- } \\
\text { second } \\
\text { samples before } \\
\text { January 1, } \\
2009 \text {, and 1- } \\
\text { minute } \\
\text { averages on } \\
\text { and after } \\
\text { January 1, } \\
2009 .\end{array}$ & $\begin{array}{c}\text { Continental United } \\
\text { States }\end{array}$ & $\begin{array}{l}\text { Seven stations: } \\
\text { Montana, } \\
\text { Colorado, Illinois, } \\
\text { Mississippi, } \\
\text { Pennsylvania, } \\
\text { Nevada, and } \\
\text { South Dakota }\end{array}$ & $\begin{array}{l}\text { GHI, DNI, DHI, downwelling infrared } \\
\text { irradiance, upwelling infrared irradiance, } \\
\text { and upwelling (reflected) shortwave } \\
\text { irradiance. Photosynthetically active } \\
\text { radiation, solar net radiation, infrared net } \\
\text { radiation, air temperature, relative } \\
\text { humidity, wind speed and direction (10 m } \\
\text { AGL), and all-sky images }\end{array}$ & $\begin{array}{l}\text { NOAA, Earth Systems Research } \\
\text { Laboratory, Global Monitoring } \\
\text { Division, in Boulder, Colorado: } \\
\text { ftp://ftp.srrb.noaa.gov/pub/data/s } \\
\text { urfrad and www.srrb.noaa.gov. } \\
\text { SURFRAD data are also } \\
\text { submitted to the BSRN archives: } \\
\text { www.bsrn.awi.de/ }\end{array}$ \\
\hline
\end{tabular}




\begin{tabular}{|c|c|c|c|c|c|c|}
\hline Database & $\begin{array}{l}\text { Period of } \\
\text { record }\end{array}$ & $\begin{array}{l}\text { Temporal } \\
\text { resolution }\end{array}$ & Spatial coverage & $\begin{array}{l}\text { Spatial } \\
\text { resolution }\end{array}$ & Data elements and sources & Availability \\
\hline $\begin{array}{l}\text { NREL's Solar } \\
\text { Radiation } \\
\text { Research } \\
\text { Laboratory Data } \\
\text { Center (MIDC) }\end{array}$ & $\begin{array}{c}1981- \\
\text { present }\end{array}$ & $\begin{array}{c}\text { 5-minute } \\
\text { (beginning July } \\
\text { 15, 1981); } 1- \\
\text { minute } \\
\text { (beginning } \\
\text { January 13, } \\
\text { 1999) }\end{array}$ & Golden, Colorado & $\begin{array}{c}\text { One } \\
\text { measurement } \\
\text { station }\end{array}$ & $\begin{array}{c}\text { GHI, DNI, DHI (from shadowband and } \\
\text { tracking disk), global on tilted surfaces, } \\
\text { reflected solar irradiance, ultraviolet, } \\
\text { infrared (upwelling and downwelling), } \\
\text { photometric and spectral radiometers, sky } \\
\text { imagery, and surface meteorological } \\
\text { conditions (temperature, relative humidity, } \\
\text { barometric pressure, precipitation, snow } \\
\text { cover, and wind speed and direction at } \\
\text { multiple levels) }\end{array}$ & $\begin{array}{c}\text { NREL Measurement \& } \\
\text { Instrumentation Data Center: } \\
\text { http://www.nrel.gov/midc/srrl bm } \\
\underline{\text { s/ }}\end{array}$ \\
\hline $\begin{array}{c}\text { NSRDB 1961- } \\
1990\end{array}$ & $1961-1990$ & Hourly & $\begin{array}{c}\text { United States and } \\
\text { territories }\end{array}$ & $\begin{array}{l}239 \text { stations }(56 \\
\text { stations have } \\
\text { some radiation } \\
\text { measurements) }\end{array}$ & $\begin{array}{l}\text { Hourly GHI, DNI, DHI, ETR, direct normal } \\
\text { ETR, total sky cover, opaque sky cover, } \\
\text { ceiling height, dry-bulb temperature, dew- } \\
\text { point temperature, relative humidity, } \\
\text { atmospheric pressure, horizontal visibility, } \\
\text { wind speed, wind direction, present } \\
\text { weather, AOD, total precipitable water, } \\
\text { snow depth, and number of days since } \\
\text { last snowfall }\end{array}$ & 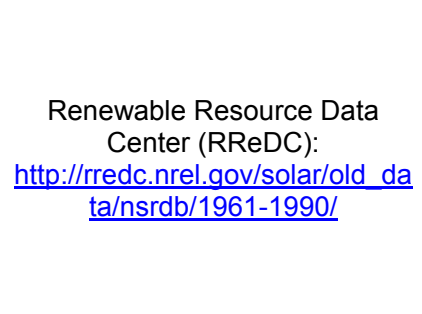 \\
\hline $\begin{array}{l}\text { NSRDB 1991- } \\
2005\end{array}$ & 1991-2005 & Hourly & United States & $\begin{array}{l}\text { 1,454 locations } \\
\text { and } 10-\mathrm{km} \text { by } 10- \\
\text { km grid (1998- } \\
2005)\end{array}$ & $\begin{array}{l}\text { Computed or modeled data: ETR on } \\
\text { surfaces horizontal and normal to the sun, } \\
\text { GHI, DNI, and DHI. Measured or observed } \\
\text { data: total sky cover, opaque sky cover, } \\
\text { dry-bulb temperature, dew-point } \\
\text { temperature, relative humidity, station } \\
\text { pressure, wind speed and direction, } \\
\text { horizontal visibility, ceiling height, } \\
\text { precipitable water, AOD, surface albedo, } \\
\text { and precipitation }\end{array}$ & $\begin{array}{c}\text { NSRDB solar fields (no } \\
\text { meteorological data); NCDC } \\
\text { distribution: } \\
\text { ftp://ftp.ncdc.noaa.gov/pub/data/ } \\
\text { nsrdb-solar/ } \\
\text { SUNY 10-km gridded data; } \\
\text { NCDC distribution: } \\
\frac{\text { ftp://ftp.ncdc.noaa.gov/pub/data/ }}{\text { nsrdb-solar/ }} \\
\text { NSRDB statistical summaries; } \\
\text { NCDC distribution: } \\
\text { ftp://ftp.ncdc.noaa.gov/pub/data/ } \\
\text { nsrdb-solar/ }\end{array}$ \\
\hline
\end{tabular}




\begin{tabular}{|c|c|c|c|c|c|c|}
\hline Database & $\begin{array}{l}\text { Period of } \\
\text { record }\end{array}$ & $\begin{array}{l}\text { Temporal } \\
\text { resolution }\end{array}$ & Spatial coverage & $\begin{array}{l}\text { Spatial } \\
\text { resolution }\end{array}$ & Data elements and sources & Availability \\
\hline $\begin{array}{l}\text { NSRDB 1991- } \\
2009\end{array}$ & 1991-2009 & Hourly & United States & $\begin{array}{l}1,454 \text { locations } \\
\text { and } 10-\mathrm{km} \text { by } 10- \\
\text { km grid (1998- } \\
2009)\end{array}$ & $\begin{array}{l}\text { Computed or modeled data: ETR on } \\
\text { surfaces horizontal and normal to the sun, } \\
\text { GHI, DNI, and DHI. Measured or observed } \\
\text { data: total sky cover, opaque sky cover, } \\
\text { dry-bulb temperature, dew-point } \\
\text { temperature, relative humidity, station } \\
\text { pressure, wind speed and direction, } \\
\text { horizontal visibility, ceiling height, } \\
\text { precipitable water, AOD, surface albedo, } \\
\text { and precipitation }\end{array}$ & $\begin{array}{c}\text { NSRDB user manual: } \\
\text { http://www.nrel.gov/docs/fy12ost } \\
\frac{\text { i/54824.pdf and }}{\text { http://RReDC.nrel.gov/solar/old }} \\
\text { data/nsrdb/1991-2010/ }\end{array}$ \\
\hline $\begin{array}{l}\text { NSRDB 1998- } \\
\quad 2015\end{array}$ & 1998-2015 & half-hourly & $\begin{array}{l}\text { Southern Canada, } \\
\text { United States, and } \\
\text { parts of South } \\
\text { America (longitude: - } \\
25^{\circ} \mathrm{E} \text { to }-175^{\circ} \mathrm{W} \text {, } \\
\text { latitude: }-20^{\circ} \mathrm{S} \text { to } 60^{\circ} \\
\mathrm{N} \text { ). }\end{array}$ & $4 \mathrm{~km}$ & $\begin{array}{l}\text { GHI, DNI, DHI, clear-sky DHI, clear-sky } \\
\text { DNI, clear-sky GHI, cloud type, dew point, } \\
\text { surface air temperature, surface pressure, } \\
\text { surface relative humidity, solar zenith } \\
\text { angle, total precipitable water, wind } \\
\text { direction, wind speed }\end{array}$ & https://nsrdb.nrel.gov \\
\hline OSI-LAF & $\begin{array}{l}2001- \\
\text { present }\end{array}$ & Hourly & $\begin{array}{l}\text { Africa, Americas, } \\
\text { Europe, western Asia }\end{array}$ & $0.05^{\circ} \times 0.05^{\circ}$ & GHI, Longwave infrared irradiance & $\begin{array}{l}\text { EUMETSAT: http://www.osi- } \\
\frac{\text { saf.org/?q=content/radiative- }}{\text { fluxes-products }}\end{array}$ \\
\hline $\begin{array}{l}\text { Pacific Northwest } \\
\text { Solar Radiation } \\
\text { Data Network }\end{array}$ & $\begin{array}{l}\text { 1977- } \\
\text { present }\end{array}$ & 5-minute & $\begin{array}{l}\text { Idaho, Montana, } \\
\text { Oregon, Utah, } \\
\text { Washington, and } \\
\text { Wyoming }\end{array}$ & $\begin{array}{l}41 \text { measurement } \\
\text { stations }\end{array}$ & $\begin{array}{c}\text { GHI, DNI, DHI, global irradiance on tilted } \\
\text { surfaces (various), spectral irradiance } \\
\text { (various), and surface meteorological data } \\
\text { (temperature, relative humidity, dew-point } \\
\text { temperature, barometric pressure, } \\
\text { precipitation, cloud cover, snow depth, } \\
\text { etc.) }\end{array}$ & $\frac{\text { http://solardat.uoregon.edu/Sola }}{\underline{\text { rData.html }}}$ \\
\hline PVGIS & $\begin{array}{l}1981- \\
\text { present }\end{array}$ & Hourly & $\begin{array}{l}\text { Europe, Africa, Asia } \\
\text { (partial) }\end{array}$ & $\begin{array}{l}\text { 1-km aggregated } \\
\text { to } 5 \text { arc-minutes } \\
(\sim 8 \mathrm{~km})\end{array}$ & $\begin{array}{c}\text { GHI, DNI, DHI, and GTI, based on the } \\
\text { CM-SAF database, optional terrain } \\
\text { shadowing }\end{array}$ & $\begin{array}{l}\text { Joint Research Center of the } \\
\text { European Commission, Institute } \\
\text { for Energy, Renewable Energy } \\
\text { Unit: } \\
\frac{\text { http://re.jrc.ec.europa.eu/pvg to }}{\text { ols/en/tools.html }}\end{array}$ \\
\hline
\end{tabular}




\begin{tabular}{|c|c|c|c|c|c|c|}
\hline Database & $\begin{array}{l}\text { Period of } \\
\text { record }\end{array}$ & $\begin{array}{l}\text { Temporal } \\
\text { resolution }\end{array}$ & Spatial coverage & $\begin{array}{l}\text { Spatial } \\
\text { resolution }\end{array}$ & Data elements and sources & Availability \\
\hline Satel-Light & $1996-2000$ & 30-minute & Europe & $\sim 5 \mathrm{~km}$ & $\begin{array}{l}\mathrm{DNI}, \mathrm{GHI}, \mathrm{DHI}, \mathrm{GTI} \text {, horizontal } \\
\text { illuminance, tilted illuminance, and sky } \\
\text { luminance distribution. }\end{array}$ & $\frac{\text { http://www.satellight.com/indexg }}{\underline{\text { S.htm }}}$ \\
\hline $\begin{array}{l}\text { Solar and Wind } \\
\text { Energy Resource } \\
\text { Assessment } \\
\text { (SWERA) }\end{array}$ & $\begin{array}{c}\text { Moderate } \\
\text { resolution: } \\
\text { 1985-1991; } \\
\text { high } \\
\text { resolution: } \\
\text { 1998-2002 }\end{array}$ & $\begin{array}{l}\text { Monthly and } \\
\text { annual average } \\
\text { daily totals } \\
\left(\mathrm{kWh} / \mathrm{m}^{2}\right.\end{array}$ & $\begin{array}{l}\text { Moderate resolution: } \\
\text { South America, } \\
\text { Central America, } \\
\text { Africa, South and } \\
\text { East Asia, Caribbean, } \\
\text { Mexico, Middle East } \\
\text { (Israel, } \\
\text { Palestine/Jordan, } \\
\text { Lebanon, Syria, Iraq, } \\
\text { Yemen, Saudi Arabia } \\
\text { [partial], and Kuwait). } \\
\text { High resolution: } \\
\text { Guatemala, Belize, El } \\
\text { Salvador, Honduras, } \\
\text { Nicaragua, partial } \\
\text { Mexico (Oaxaca), } \\
\text { Cuba, Afghanistan, } \\
\text { Pakistan, partial } \\
\text { Mexico (Chiapas, } \\
\text { Vera Cruz, northern } \\
\text { Mexico to 24 degrees } \\
\text { latitude), Dominican } \\
\text { Republic, Bhutan, } \\
\text { India (NW), Ethiopia, } \\
\text { Ghana, Kenya, Sri } \\
\text { Lanka, Nepal, } \\
\text { Bangladesh, Western } \\
\text { China, and United } \\
\text { Arab Emirates }\end{array}$ & $\begin{array}{l}\text { Moderate } \\
\text { resolution: } 40 \mathrm{~km} \text {; } \\
\text { high resolution: } 10 \\
\mathrm{~km}\end{array}$ & $\begin{array}{l}\text { GHI, DNI (DHI), and GTI from model } \\
\text { estimates based on surface } \\
\text { meteorological observations and/or } \\
\text { satellite remote-sensing input data }\end{array}$ & $\begin{array}{l}\text { Designed by the Solar and Wind } \\
\text { Energy Resource Assessment } \\
\text { program and maintained by } \\
\text { UNEP/GRID-Sioux Falls: } \\
\text { - http://maps.nrel.gov/swera } \\
\text { - Products for Brazil were } \\
\text { developed by Brazil's National } \\
\text { Institute of Space Research and } \\
\text { Laboratory of Solar } \\
\text { Energy/Federal University of } \\
\text { Santa Catarina. More } \\
\text { information about INPE is } \\
\text { available at } \\
\text { http://www.inpe.br/ingles/index.p } \\
\text { hp. - Products developed by } \\
\text { DLR are available from } \\
\text { http://www.dlr.de/tt/desktopdefa } \\
\underline{\text { ult.aspx/tabid-2885/4422 read- }} \\
\underline{6548 /}\end{array}$ \\
\hline $\begin{array}{l}\text { Solar Data } \\
\text { Warehouse }\end{array}$ & $\begin{array}{l}\text { Varies from } \\
5-25 \text { years } \\
\text { ago to the } \\
\text { present }\end{array}$ & $\begin{array}{l}\text { Hourly and } \\
\text { daily }\end{array}$ & $\begin{array}{c}\text { Continental United } \\
\text { States }\end{array}$ & $\begin{array}{l}\text { More than } 3,000 \\
\text { measurement } \\
\text { stations }\end{array}$ & $\mathrm{GHI}$ & $\frac{\text { http://www.solardatawarehouse. }}{\underline{\text { com/ }}}$ \\
\hline
\end{tabular}




\begin{tabular}{|c|c|c|c|c|c|c|}
\hline Database & $\begin{array}{l}\text { Period of } \\
\text { record }\end{array}$ & $\begin{array}{l}\text { Temporal } \\
\text { resolution }\end{array}$ & Spatial coverage & $\begin{array}{l}\text { Spatial } \\
\text { resolution }\end{array}$ & Data elements and sources & Availability \\
\hline $\begin{array}{l}\text { Solar Energy and } \\
\text { Meteorological } \\
\text { Research Training } \\
\text { Sites }\end{array}$ & $1979-1983$ & 1-minute & $\begin{array}{l}\text { Fairbanks, Alaska; } \\
\text { Atlanta, Georgia; } \\
\text { Albany, New York; } \\
\text { San Antonio, Texas }\end{array}$ & $\begin{array}{l}\text { Four } \\
\text { measurement } \\
\text { stations }\end{array}$ & $\begin{array}{c}\text { GHI, DNI, and DHI; GTI on various } \\
\text { surfaces, infrared irradiances, ultraviolet } \\
\text { and other spectral irradiance (varies), and } \\
\text { surface meteorological conditions } \\
\text { (temperature, relative humidity, pressure, } \\
\text { visibility, wind speed, and direction at } 10 \\
\text { m, precipitation, etc.) }\end{array}$ & $\begin{array}{c}\text { NREL's RReDC: } \\
\frac{\text { http://rredc.nrel.gov/solar/old da }}{\underline{\text { ta/semrts/ }}}\end{array}$ \\
\hline SolarGIS & $\begin{array}{l}1994,1999, \\
2007- \\
\text { present } \\
\text { (depends on } \\
\text { region) } \\
\end{array}$ & $\begin{array}{l}\text { 15- and 30- } \\
\text { minute }\end{array}$ & $\begin{array}{l}\text { Land area, worldwide, } \\
\text { between latitudes } 60^{\circ} \\
\mathrm{N} \text { and } 50^{\circ} \mathrm{S}\end{array}$ & $\begin{array}{c}\sim 3 \mathrm{~km} \text { (at the } \\
\text { equator) down } \\
\text { scaled to } \sim 80 \mathrm{~m} \\
\text { using SRTM-3 } \\
\text { DEM } \\
\end{array}$ & $\begin{array}{l}\text { DNI, GHI, DHI, GTI, and air temperature } \\
(2-\mathrm{m} \mathrm{AGL}) \text { and others }\end{array}$ & http://solargis.info \\
\hline SOLDAY & $\begin{array}{l}\text { January } \\
1952- \\
\text { December } \\
1976\end{array}$ & Daily & $\begin{array}{l}\text { Continental United } \\
\text { States }\end{array}$ & $\begin{array}{l}26 \text { measurement } \\
\text { stations }\end{array}$ & $\begin{array}{l}\text { Computed times of daily sunrise and } \\
\text { sunset; ETR (based on solar constant = } \\
\left.1,377 \mathrm{~W} / \mathrm{m}^{2}\right) \text {; measured GHI from } \\
\text { mechanical integrators and strip charts } \\
\text { and daily amounts calculated by summing } \\
\text { hand-computed hourly values, minutes, } \\
\text { and percent of possible sunshine; } \\
\text { temperature (maximum, minimum, mean), } \\
\text { precipitation, snowfall, snow depth, } \\
\text { weather codes, and sky cover from hourly } \\
\text { observations; None of the } 26 \text { SOLDAY } \\
\text { measurement stations are in the hourly } \\
\text { SOLMET data set. }\end{array}$ & $\begin{array}{l}\text { NCDC, NOAA, NESDIS, U.S. } \\
\text { Department of Commerce: } \\
\text { http://www.ncdc.noaa.gov/ }\end{array}$ \\
\hline SOLEMI & $\begin{array}{c}1991- \\
\text { present }\end{array}$ & 30-minute & $\begin{array}{l}\text { Europe, Africa, South } \\
\text { America, Western } \\
\text { Asia, Western } \\
\text { Australia }\end{array}$ & $2.5 \mathrm{~km}$ & GHI, DNI & $\begin{array}{c}\text { DLR: } \\
\frac{\text { http://www.dlr.de/tt/en/desktopd }}{\text { efault.aspx/tabid- }} \\
\underline{\text { 2885/4422 read-6581/ }}\end{array}$ \\
\hline SOLMET/ERSATZ & $\begin{array}{l}\text { December } \\
1951- \\
\text { December } \\
1976\end{array}$ & $\begin{array}{l}\text { Hourly (hour } \\
\text { ending in LST) }\end{array}$ & $\begin{array}{l}\text { United States and } \\
\text { territories }\end{array}$ & $\begin{array}{l}26 \text { measurement } \\
\text { stations and } 222 \\
\text { modeled stations }\end{array}$ & $\begin{array}{l}\text { ETR, GHI (observed-SOLMET or } \\
\text { modeled-ERSATZ, engineering corrected, } \\
\text { standard-year corrected), DNI (estimated } \\
\text { from GHI), minutes of sunshine, clouds } \\
\text { (ceiling height, total and opaque cloud } \\
\text { fractions, and information for up to four } \\
\text { cloud layers), and surface meteorological } \\
\text { conditions (temperature, wind speed, } \\
\text { pressure, snow cover, horizontal visibility, } \\
\text { sky condition, and current weather) }\end{array}$ & $\begin{array}{c}\text { NCDC, NOAA, NOAA's Satellite } \\
\text { and Information Service } \\
\text { (NESDIS), U.S. Department of } \\
\text { Commerce: } \\
\text { http://www.ncdc.noaa.gov/ }\end{array}$ \\
\hline
\end{tabular}

\section{$5-14$}

This report is available at no cost from the National Renewable Energy Laboratory at www.nrel.gov/publications. 


\begin{tabular}{|c|c|c|c|c|c|c|}
\hline Database & $\begin{array}{l}\text { Period of } \\
\text { record }\end{array}$ & $\begin{array}{l}\text { Temporal } \\
\text { resolution }\end{array}$ & Spatial coverage & $\begin{array}{l}\text { Spatial } \\
\text { resolution }\end{array}$ & Data elements and sources & Availability \\
\hline TMY 98-15 & 1998-2015 & Hourly & $\begin{array}{c}\text { Southern Canada, } \\
\text { United States, and } \\
\text { parts of South } \\
\text { America (longitude: - } \\
25^{\circ} \mathrm{E} \text { to }-175^{\circ} \mathrm{W}, \\
\text { latitude: }-20^{\circ} \mathrm{S} \text { to } 60^{\circ} \\
\mathrm{N} \text { ). }\end{array}$ & $4 \mathrm{~km}$ & $\begin{array}{l}\mathrm{GHI}, \mathrm{DNI}, \mathrm{DHI} \text {, cloud type, dew point, } \\
\text { surface air temperature, surface pressure, } \\
\text { wind direction, wind speed }\end{array}$ & https://nsrdb.nrel.gov \\
\hline TMY2 & $\begin{array}{l}\text { One year } \\
\text { representati } \\
\text { ve of the } \\
\text { 1961-1990 } \\
\text { NSRDB data } \\
\text { period }\end{array}$ & Hourly & $\begin{array}{c}\text { United States and } \\
\text { territories }\end{array}$ & $\begin{array}{l}239 \text { stations } \\
\text { representing the } \\
1961-1990 \\
\text { NSRDB }\end{array}$ & Same as NSRDB 1961-1991 & $\begin{array}{c}\text { NREL RReDC: } \\
\frac{\text { http://rredc.nrel.gov/solar/old da }}{\underline{\text { ta/nsrdb/1961-1990/tmy2/ }}}\end{array}$ \\
\hline TMY3 & 1991-2005 & Hourly & $\begin{array}{c}\text { United States and } \\
\text { territories }\end{array}$ & 1,020 locations & $\begin{array}{l}\text { Computed or modeled data: ETR on } \\
\text { surfaces horizontal and normal to the sun, } \\
\text { GHI and illuminance, DNI and illuminance, } \\
\text { DHI and illuminance, zenith luminance. } \\
\text { Measured or observed data: total sky } \\
\text { cover, opaque sky cover, dry-bulb } \\
\text { temperature, dew-point temperature, } \\
\text { relative humidity, station pressure, wind } \\
\text { speed and direction, horizontal visibility, } \\
\text { ceiling height, precipitable water, AOD, } \\
\text { surface albedo, and precipitation }\end{array}$ & $\begin{array}{c}\text { The NREL RReDC: } \\
\text { http://RReDC.nrel.gov/solar/old } \\
\text { data/nsrdb/1991-2005/tmy3/. }\end{array}$ \\
\hline $\begin{array}{l}\text { Western Energy } \\
\text { Supply and } \\
\text { Transmission } \\
\text { Associates Solar } \\
\text { Monitoring } \\
\text { Network }\end{array}$ & $1976-1980$ & 15-minute & $\begin{array}{l}\text { Arizona, California, } \\
\text { Colorado, Nevada, } \\
\text { New Mexico, and } \\
\text { Wyoming }\end{array}$ & $\begin{array}{l}52 \text { measurement } \\
\text { stations }\end{array}$ & $\begin{array}{c}\text { GHI, DNI, and dry-bulb temperature } \\
\text { measured with pyranometers (Eppley } \\
\text { Black and White, Eppley PSP, and the } \\
\text { Spectrolab Spectrosun SR75) and } \\
\text { pyrheliometers (Eppley NIP) in automatic } \\
\text { solar trackers. }\end{array}$ & $\begin{array}{c}\text { NREL RReDC: } \\
\frac{\text { http://rredc.nrel.gov/solar/pubs/w }}{\underline{\text { a/wa index.html }}}\end{array}$ \\
\hline
\end{tabular}




\begin{tabular}{|c|c|c|c|c|c|c|}
\hline Database & $\begin{array}{l}\text { Period of } \\
\text { record }\end{array}$ & $\begin{array}{l}\text { Temporal } \\
\text { resolution }\end{array}$ & Spatial coverage & $\begin{array}{l}\text { Spatial } \\
\text { resolution }\end{array}$ & Data elements and sources & Availability \\
\hline $\begin{array}{l}\text { World } \\
\text { Meteorological } \\
\text { Organization } \\
\text { WRDC }\end{array}$ & $\begin{array}{c}1964- \\
\text { present }\end{array}$ & $\begin{array}{l}\text { Daily totals with } \\
\text { some hourly } \\
\text { measurements } \\
\text { at a few sites }\end{array}$ & Global & $\begin{array}{l}\text { More than } 1,000 \\
\text { measurement } \\
\text { stations }\end{array}$ & $\begin{array}{l}\text { Primarily daily total GHI, radiation balance, } \\
\text { and sunshine duration, but some } \mathrm{DHI} \text { and } \\
\text { DNI. Some hourly measurements are } \\
\text { available from a few sites. }\end{array}$ & $\frac{\text { http://wrdc-mgo.nrel.gov }}{\text { http://wrdc.mgo.rssi.ru }}$ \\
\hline $\begin{array}{l}\text { National Center for } \\
\text { Environmental } \\
\text { Protection/National } \\
\text { Center for } \\
\text { Atmospheric } \\
\text { Research Global } \\
\text { Reanalysis } \\
\text { Products (NCEP) }\end{array}$ & 1948-2009 & 6 -hour $\left(\mathrm{W} / \mathrm{m}^{2}\right)$ & Global & $2.5^{\circ}$ (nominal) & $\begin{array}{l}\mathrm{GHI} \text { and more than } 80 \text { variables, including } \\
\text { geopotential height, temperature, relative } \\
\text { humidity, and } U \text { and } \mathrm{V} \text { wind components, } \\
\text { in several coordinate systems, such as a } \\
\text { 17-pressure-level stack on } 2.5 \times 2.5^{\circ} \text { grids, } \\
28 \text { sigma-level stacks on } 192 \text { by } 94 \\
\text { Gaussian grids, and } 11 \text { isentropic-level } \\
\text { stacks on a } 2.5 \times 2.5^{\circ} \text { grid }\end{array}$ & $\begin{array}{c}\text { University Center for } \\
\text { Atmospheric Research, } \\
\text { Computational and Information } \\
\text { Systems Laboratory Research } \\
\text { Data Archive: } \\
\frac{\text { http://rda.ucar.edu/datasets/ds0 }}{\underline{90.0 /}}\end{array}$ \\
\hline
\end{tabular}




\section{References}

Arguez, A., and R. S. Vose. 2011. "The Definition of the Standard WMO Climate Normal: The Key to Deriving Alternative Climate Normals." Bulletin of the American Meteorological Society 92: 699-704.

Gueymard, C. A., and D. R. Myers. 2008. "Solar Radiation Measurement: Progress in Radiometry for Improved Modeling.” In: Modeling Solar Radiation at the Earth Surface, V. Badescu (Ed.), Springer, pp. 1-27.

—. 2009. "Solar Resource for Space and Terrestrial Applications." In: Solar Cells and their Applications, L. Fraas and L. Partain (Eds.), Wiley, pp. 427-461.

Hall, I., R. Prairie, H. Anderson, and E. Boes. 1978. Generation of Typical Meteorological Years for 26 SOLMET Stations. SAND78-1601. Albuquerque, NM: Sandia National Laboratories.

Hulstrom, R. L., ed. 1989. Solar Resources. Cambridge, MA: MIT Press.

Marion, W., and K. Urban. 1995. User's Manual for TMY2s-Typical Meteorological Years Derived from the 1961-1990 National Solar Radiation Database. NREL/TP-463-7668. Golden, CO: National Renewable Energy Laboratory.

Sengupta, M., A. Habte, P. Gotseff, A. Weekley, A. Lopez, M. Anderberg, C. Molling, and A. Heidinger. 2014. "Physics-Based GOES Product for Use in NREL's National Solar Radiation Database." Preprint. Prepared for the European Photovoltaic Solar Energy Conference and Exhibition, Sept. 22-26, 2014. NREL/CP-5D00-62776. Golden, CO: National Renewable Energy Laboratory.

Wilcox, S. 2007. National Solar Radiation Database 1991-2005 Update: User's Manual. NREL/TP-581-41364. Golden, CO: National Renewable Energy Laboratory. 


\title{
6 Measurement and Model Uncertainty
}

\author{
Aron Habte, ${ }^{1}$ Daryl Myers, ${ }^{2}$ Stefan Wilbert, ${ }^{3}$ Marcel Suri, ${ }^{4}$ Manajit \\ Sengupta, ${ }^{1}$ Thomas Stoffel, ${ }^{5}$ Frank Vignola, ${ }^{6}$ and Anthony Lopez ${ }^{1}$ \\ 1. National Renewable Energy Laboratory \\ 2. Retired, National Renewable Energy Laboratory \\ 3. German Aerospace Center (DLR) \\ 4. Solargis \\ 5. Solar Resource Solutions, $L L C$ \\ 6. University of Oregon
}

To fully characterize measured or modeled solar resource data, the data set should be accompanied by a statement of uncertainty that will help the analyst to correctly apply the information and will provide the necessary context for the reliability of each value. For example, a full characterization of uncertainty provides a basis to assess the predicted output of planned solar conversion systems and is thus a key factor when determining the bankability of the project. Uncertainty can be thought of as the confidence one has in the data. However, it is important to determine the uncertainty using a standard methodology that others also can use and will obtain identical results. The Guide to the Expression of Uncertainty in Measurements (GUM) (ISO 2008) is an example of how to determine the uncertainty in measurements. GUM has been formalized by several organizations, including the International Bureau of Weights and Measurements (French acronym: BIPM), and published by the International Standards Organization (ISO).

In this chapter, the uncertainties associated with the measured or modeled solar resource data are discussed along with the validation of physical or empirical models that use such data. Precise methods to measure and model the solar resource are difficult to develop because of the rapidly changing nature of solar irradiance. While instrumentation is improving, the measurement or modeling of incident irradiance can have large uncertainties, depending on circumstances. The GUM methodology for quantifying uncertainty in either measured (Section 6.1) or modeled values (Section 6.2) is discussed in what follows. Note that the uncertainty in modeled data is typically obtained by comparison with reference measurements, which is why this development comes first.

\subsection{Measurement Uncertainty}

To characterize a quantity, referred to in the GUM terminology as the measurand, it is necessary to provide a measure of the quantity. This characterization of the measurand is incomplete without supplying the associated uncertainty. This uncertainty provides an estimate of how well the value of the measurand is known and provides a range of values that will result from measurements taken under similar circumstances with similar instruments. In general, the measurand has four general sources of uncertainty: the act of measurement, the instrument doing the measurement, the device recording the measurement, and the environment in which the measurements take place.

Every measurement only approximates the quantity being measured, and it is incomplete without a quantitative statement of uncertainty. Each element of a measurement system contributes to the final uncertainty of the data. Accurate measurements of solar radiation made at ground stations depend on the radiometer specifications, proper installation and maintenance, data acquisition 
method and accuracy, calibration method and frequency, location, environmental conditions, and possible real-time or a posteriori corrections to the data. A large portion of this overview of uncertainty in measurements of solar radiation made at ground stations is based on Habte et al. (2014, 2016b), Reda et al. (2011), Wilcox and Myers (2008), Myers et al. (2002), Stoffel et al. (2000), and Gueymard and Myers (2008a).

\subsubsection{Estimation of Calibration and Field Measurement Uncertainty}

The method to estimate uncertainty has changed significantly during the last few decades. Unfortunately, outdated terminology and methods are still used at times. This section starts with a short description of the outdated methodology, which is not recommended. This will help users, however, if the uncertainty of data is presented using older methodologies.

Historically, uncertainty analysis treated sources of uncertainty in terms of random and bias error types. Random sources were related to the standard deviation or variance of measured data sets. Biases were estimates of deviations from a "true value" primarily based on engineering judgments of the measurement system performance. Total uncertainty (UT $\mathrm{Uld}_{\text {old }}$ ) was computed as the square root of the sum of the squares for these two error types.

$$
\mathrm{UT}_{\mathrm{old}}=\left[\Sigma(\mathrm{Bias})^{2}+\Sigma\left(2 \cdot \mathrm{Random}^{2}\right]^{1 / 2}\right.
$$

The factor 2 in the random term was necessary to "inflate" the random component and ultimately provide an approximate $95 \%$ confidence interval $(k \approx 2$, for infinite degrees of freedom) for the computed value of $\mathrm{UT}_{\text {old }}$. This assumes the data points are normally distributed (i.e., random). Based on advancement in metrology science, this simple method is now replaced by a more elaborate one.

GUM is currently the accepted guide for measurement uncertainty (ISO 2008). Similarly, the method provides the expanded uncertainty for an approximate $95 \%$ confidence interval by multiplying the combined uncertainty by the coverage factor $k(k=1.96$, sometimes approximated as 2 for infinite degrees of freedom). GUM defines Type $A$ uncertainty values as derived from statistical methods and Type $B$ sources as evaluated by other means, such as scientific judgment, experience, specifications, comparisons, and calibration data. GUM defines the concept of a standard uncertainty $\left(\mathrm{u}_{\text {std }}\right)$ for each uncertainty type, which is an estimate of an equivalent standard deviation (of a specified distribution) of the source of uncertainty. In order to appropriately combine the various uncertainties, the GUM methodology uses a sensitivity coefficient (c) that is calculated from the measurement equation using partial derivatives with respect to each input variable in the equation. The combined uncertainty $\left(u_{c}\right)$ is computed from the Type A and Type B standard uncertainties summed under quadrature - the square root of the sum of the squares. GUM removes the historical factor of 2 and rather introduces the coverage factor $k$ (which depends on the known or assumed statistical distribution of uncertainties) ${ }^{1}$, which is applied to both types of uncertainty in order to compute the expanded uncertainty $\left(\mathrm{U}_{\mathrm{E}}\right)$ as:

\footnotetext{
${ }^{1} k$ is 1.96 for a Gaussian distribution for a $95 \%$ confidence level. Roughly, a $95 \%$ confidence level means that $95 \%$ of the values will be with the statistical limits defined by the uncertainty.
} 


$$
\mathrm{U}_{\mathrm{E}}=k \cdot \mathrm{u}_{\mathrm{c}}=k \cdot\left[\Sigma(\text { Type } \mathrm{B})^{2}+\Sigma(\text { Type } \mathrm{A})^{2}\right]^{1 / 2}
$$

\section{The combined uncertainty times the coverage factor ( $k$ = 1.96 for a $95 \%$ confidence interval)}

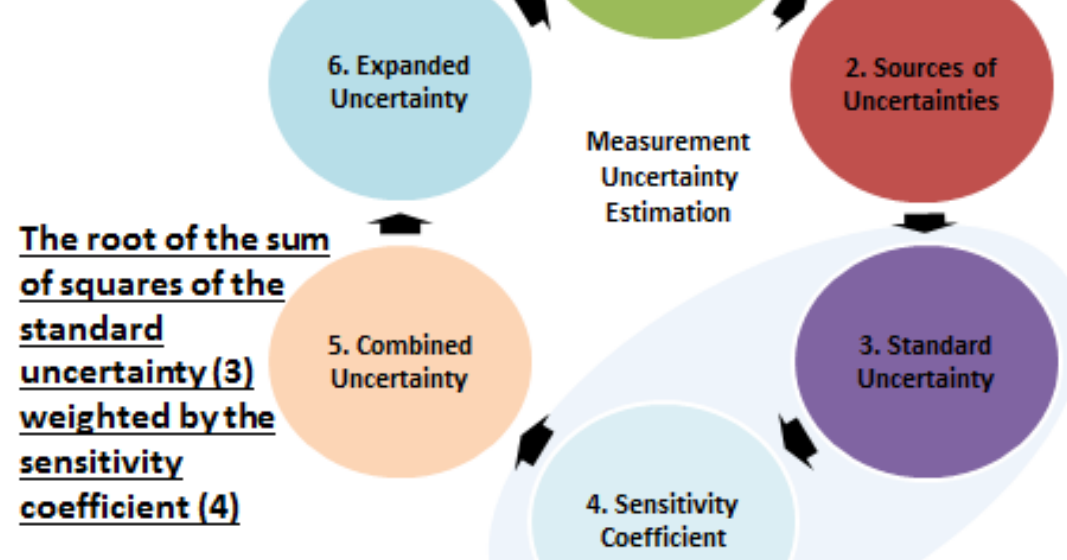

Expanded Uncertainty from given sources of uncertainties [Type B]] Uncertainties derived from statistical analysis of measurements [Type A]

\section{Partial derivative for each variable in the measurement equation}

Figure 6-1. Measurement uncertainty estimation flow chart (Habte et al. 2016b)

As shown in Figure 6-1, the GUM procedure can be summarized in six steps (Habte et al. 2016b Reda 2011):

1. Define the measurement equation for the calibration and/or measurement system: This consists of a mathematical description of the relation between sensor voltage and any other independent variables and the desired output (calibration response or engineering units for measurements). The two example equations used to quantify radiometric measurement are:

$$
E=\frac{\left(V-R_{n e t} * W_{n e t}\right)}{R} \quad \text { or } \quad E=\frac{V}{R}
$$

where

$E=$ irradiance, in $\mathrm{Wm}^{-2}$ (global horizontal irradiance [GHI], global tilted irradiance [GTI], diffuse horizontal irradiance [DHI], or direct normal irradiance [DNI]); for DNI, $R_{n e t}=0$, resulting in the equation on the right

$R=$ responsivity of the radiometer in $\mu \mathrm{V} /\left(\mathrm{Wm}^{-2}\right)$

$V=$ sensor output voltage of the radiometer in $\mu \mathrm{V}$

$R_{\text {net }}=$ net infrared responsivity of the radiometer in $\mu \mathrm{V} /\left(\mathrm{Wm}^{-2}\right)$

$W_{n e t}=$ effective net infrared irradiance measured by a collocated pyrgeometer in $\mathrm{Wm}^{-2}$

In the case of $\mathrm{GHI}$, the closure equation applies: $E=D N I * \operatorname{Cos}(Z)+D H I$, where 
$D N I=$ beam irradiance measured by a primary or standard reference standard pyrheliometer in $\mathrm{Wm}^{-2}$

$Z=$ solar zenith angle (SZA), in degrees or radians

$D H I=$ diffuse horizontal irradiance, measured by a shaded pyranometer $\left(\mathrm{Wm}^{-2}\right)$.

2. Determine sources of uncertainties: Most of the sources of uncertainties are obtained from statistical calculations, specifications from manufacturers, and previously published reports on radiometric data uncertainty or professional experience. Some of the sources of the common uncertainties come from solar zenith angular response, spectral response, non-linearity, temperature response, thermal loss, data logger accuracy, soiling, and calibration including the drift of the calibration constant(s).

3. Calculate standard uncertainty, $\boldsymbol{u}$ : In this step, an individual $u$ for each variable in the measurement equation is calculated, using either statistical methods (Type A uncertainty component) or other methods (Type B uncertainty component, such as manufacturer specifications, calibration results, and experimental or engineering experience). In the GUM method, the standard uncertainties are calculated by dividing the expanded uncertainty of each source by the corresponding statistical distribution (ASTM G213-17).

A. TYPE A uncertainty

i. Type A standard uncertainty is calculated by taking repeated measurements of the input quantity value, from which the sample mean and sample standard deviation $(S D)$ can be calculated. The Type A standard uncertainty $(u)$ can then be estimated by:

$$
S D=\sqrt{\frac{\sum_{i=1}^{n}\left(X_{i}-\bar{X}\right)^{2}}{n-1}}
$$

B. TYPE B uncertainty

i. Equation for unknown statistical distribution (common assumption: rectangular distribution): $u=\frac{U}{\sqrt{3}}$ where $U$ is the expanded uncertainty of a variable

ii. Normal distribution: $u=\frac{U}{k}$ where $k$ is a coverage factor of 2 or, more exactly, 1.96 (ISO 2008) iii. For other statistical distributions, other values are used for $k$.

4. Compute sensitivity coefficient, $\boldsymbol{c}$ : To appropriately combine the various uncertainties in the next step, the uncertainties must be weighed. The GUM method does this by calculating the sensitivity coefficients $(c)$ of the variables in a measurement equation. These coefficients affect the contribution of each input factor to the combined uncertainty of the irradiance value. Therefore, the sensitivity coefficient for each input is calculated by partial differentiation with respect to each input variable in the measurement equation. Table 6-1 shows sensitivity coefficients for the measurement equation (6-3). 
The sensitivity equations given in Table 6-1 are for two distinct situations. The calibration sensitivity is for calibrations when the GHI is calculated from reference DNI and DHI measurements. The second column is for GHI measurements in the field. The calibration sensitivities are related to the inverse of the GHI value, while the field sensitivities are related to the inverse of the responsivity.

Table 6-1. Example of Computing Sensitivity Coefficient Using Partial Derivative

Calibration Sensitivity Equations

$$
\begin{gathered}
c_{V}=\frac{\partial R}{\partial V}=\frac{1}{D N I \operatorname{Cos}(S Z A)+D H I} \\
c_{R n e t}=\frac{\partial R}{\partial R_{n e t}}=\frac{-W_{n e t}}{D N I \operatorname{Cos}(S Z A)+D H I} \\
c_{W n e t}=\frac{\partial R}{\partial W_{n e t}}=\frac{-R_{n e t}}{D N I \operatorname{Cos}(S Z A)+D H I} \\
c_{D N I}=\frac{\partial R}{\partial D N I}=\frac{-\left(V-R_{n e t} W_{n e t}\right) \operatorname{Cos}(S Z A)}{(D N I \operatorname{Cos}(S Z A)+D H I)^{2}} \\
c_{S Z A}=\frac{\partial R}{\partial S Z A}=\frac{D N I \operatorname{Sin}(S Z A)\left(V-R_{n e t} W_{n e t}\right)}{(N \operatorname{Cos}(Z)+D H I)^{2}} \\
c_{D}=\frac{\partial R}{\partial D H I}=\frac{-\left(V-R n e t W_{n e t}\right)}{(D N I \operatorname{Cos}(S Z A)+D H I)^{2}}
\end{gathered}
$$

Field Measurement Sensitivity Equations

$\mathrm{c}_{\mathrm{R}}=\frac{\partial \mathrm{G}}{\partial \mathrm{R}}=\frac{-\left(\mathrm{V}-R_{n e t} W_{n e t}\right)}{\mathrm{R}^{2}}$$$
\mathrm{C}_{R_{n e t}}=\frac{\partial \mathrm{G}}{\partial R_{n e t}}=\frac{-W_{\text {net }}}{\mathrm{R}}
$$$$
\mathrm{C}_{W_{\text {net }}}=\frac{\partial G H I}{\partial W_{\text {net }}}=\frac{-R_{\text {net }}}{\mathrm{R}}
$$

$$
\mathrm{c}_{V}=\frac{\partial \mathrm{GHI}}{\partial \mathrm{V}}=\frac{1}{\mathrm{R}}
$$

5. Combined standard uncertainty, $\boldsymbol{u}_{\boldsymbol{c}}$ : This is the combined standard uncertainty using the propagation of errors formula and quadrature (root sum of squares) method. It is applicable to both Type A and Type B sources of uncertainties. Standard uncertainties $(u)$ multiplied by their sensitivity factors $(c)$ are combined in quadrature to give the combined standard uncertainty, $u_{c}$.

$$
u_{c}=\sqrt{\sum_{j=0}^{n-1}(u * c)^{2}}
$$

where $n$ is the number of uncertain variables that are used to calculate the combined uncertainty.

6. Calculate the expanded uncertainty $\left(U_{95}\right)$ : The expanded uncertainty is calculated by multiplying the combined standard uncertainty by the coverage factor, typically by applying Student $t$-analysis to determine the appropriate value of $k$ (typically 1.96 for $95 \%$ and 3 for $98 \%$ confidence, respectively, for large data sets assuming a Gaussian distribution).

$$
U_{95}=k * u_{c}(6-6)
$$


The above six steps are applicable to the quantification of the uncertainty of both calibration and field measurements. Uncertainty in measurements begins with the uncertainty in calibration references, calibration processes, and sensor design characteristics. For example, for thermopile sensors, a calibration constant is required to convert the output voltage to the required irradiance, as discussed in Chapter 3. The resulting uncertainty in calibration factors must then be combined with the influence of additional sources of uncertainty in the field measurement instrumentation, installation methods, data acquisition, and operation and maintenance (O\&M) processes (Reda 2011).

The measurement of terrestrial solar radiation is traceable to the internationally accepted World Radiometric Reference (WRR) (ISO 1990), as discussed in Chapter 3. This internationally recognized measurement reference is a detector-based standard maintained by a group of electrically self-calibrating absolute cavity radiometers. The present accepted inherent expanded uncertainty in the WRR is $\pm 0.30 \%$ (Finsterle 2011). Reference cavity radiometers used as national and institutional standards are calibrated by comparison to the World Standard Group of absolute cavity pyrheliometers. Transfer of calibrations from the WRR to national standards results in an expanded uncertainty for these measurement standards of $\pm 0.45 \%$ (Reda et al. 2013).

Applying the GUM procedure to the case of pyrheliometer calibration, Table 6-2 summarizes the estimated uncertainties that are typically found in practice.

Table 6-2. Example of Estimated Pyrheliometer Calibration Uncertainties

\begin{tabular}{|c|c|c|c|}
\hline Type A Error Source & Ustd (\%) & Type B Error Source & Ustd $(\%)$ \\
\hline WRR transfer & 0.200 & WRR uncertainty (UE, $k=2)$ & 0.300 \\
\hline $\begin{array}{l}\text { Absolute cavity responses to } \\
\text { environmental conditions }\end{array}$ & 0.013 & $\begin{array}{l}\text { Absolute cavity bias } \\
\text { responses to environmental } \\
\text { conditions }\end{array}$ & 0.013 \\
\hline Data logger precision & 0.003 & Data logger bias $(9 \mu \mathrm{V} / 10 \mathrm{mV})$ & 0.090 \\
\hline $\begin{array}{l}\text { Pyrheliometer detector } \\
\text { temperature response }\end{array}$ & 0.250 & $\begin{array}{l}\text { Pyrheliometer detector } \\
\text { temperature response }\end{array}$ & 0.250 \\
\hline Pyrheliometer detector linearity & 0.100 & $\begin{array}{l}\text { Event-to-event temperature } \\
\text { bias }\left(10^{\circ} \mathrm{C}\right)\end{array}$ & 0.125 \\
\hline $\begin{array}{l}\text { Solar tracker alignment } \\
\text { variations }\end{array}$ & 0.125 & Solar tracker alignment bias & 0.125 \\
\hline $\begin{array}{l}\text { Pyrheliometer window spectral } \\
\text { transmittance }\end{array}$ & 0.500 & $\begin{array}{l}\text { Pyrheliometer window } \\
\text { spectral transmittance }\end{array}$ & 0.500 \\
\hline $\begin{array}{l}\text { Electromagnetic interference and } \\
\text { electromagnetic field }\end{array}$ & 0.005 & $\begin{array}{l}\text { Electromagnetic interference } \\
\text { and electromagnetic field }\end{array}$ & 0.005 \\
\hline
\end{tabular}

In addition, Table 6-3 identifies the typical sources of uncertainty considered for the overall uncertainty analysis of DNI measurements from two types of radiometers (pyrheliometers with thermopiles [TP]; rotating shadowband irradiometers [RSIs] with silicon [Si] pyranometer). Note that the contribution to uncertainty caused by insufficient maintenance (alignment, leveling, 
cleaning) can be much greater than the combined uncertainties for well-maintained instruments. As explained in Chapter 3, instruments with clear optics are more strongly affected by soiling. Hence, the uncertainty related to their operation in the field depends on the regularity and quality of their maintenance over time.

Accounting for the calibration uncertainty and other sources of measurement errors (e.g., condition of the radiometer optics or relevant issues similar to those considered for the estimates of uncertainty in calibration measurement), the expanded measurement uncertainty for subhourly DNI measurements is $\pm 1.5 \%- \pm 2.5 \%$ for a well-maintained measurement station equipped with a thermopile-based pyrheliometer and $\pm 5 \%$ for a photodiode-based RSI with state-of-the-art correction functions for systematic errors (Wilcox and Myers 2008).

For RSI instruments, one of the most crucial impacts on uncertainty is the spectral irradiance error. This is because Si-photodiode sensors only detect visible and infrared radiation in the 300 $1,200 \mathrm{~nm}$ range and have a spectral response that varies strongly within this wavelength interval. Furthermore, the role of using algorithms to reduce systematic effects and the uncertainty introduced by imperfect shading must be considered. A more detailed uncertainty analysis for RSIs following GUM can be found in Wilbert et al. (2016). The study defines a method for the derivation of the spectral error and spectral uncertainties and presents quantitative values of the spectral and overall uncertainties. The results of this detailed analysis are close to those presented in Table 6-3. For GHI, slightly lower uncertainties than for DNI were found (4\%, $k=$ 2 , after application of state-of-the-art correction functions). Advanced correction functions were found to significantly reduce the uncertainty.

A detailed uncertainty analysis for high-quality field pyranometers and pyrheliometers can be found in Vuilleumier et al. (2014). 
Table 6-3. Example of Estimated DNI Sub-Hourly Measurement Uncertainties (\%)

\begin{tabular}{|c|c|c|c|c|c|}
\hline Type A Error Source & $\underset{\mathrm{u}_{\text {std }}(\%)}{\mathrm{TP}^{\mathrm{a}}}$ & $\begin{array}{l}\mathrm{u}_{\text {std }} \\
(\%) \\
\mathrm{RSI}^{\mathrm{b}}\end{array}$ & Type B Error Source & $\begin{array}{l}\mathrm{u}_{\text {std }} \\
(\%) \\
\mathrm{TP}^{\mathrm{a}}\end{array}$ & $\begin{array}{c}\mathrm{u}_{\text {std }}(\%) \\
\mathrm{RSI}^{\mathrm{b}}\end{array}$ \\
\hline $\begin{array}{l}\text { Fossilized }^{\mathrm{C}} \text { calibration } \\
\text { error }\end{array}$ & 0.615 & 0.615 & Fossilized calibration error & 0.665 & 0.665 \\
\hline $\begin{array}{l}\text { Data logger precision }( \pm 50 \\
\mu \mathrm{V} / 10 \mathrm{mV})^{\mathrm{d}}\end{array}$ & 0.500 & 0.500 & $\begin{array}{l}\text { Data logger bias } \\
(1.7 \mu \mathrm{V} / 10 \mathrm{mV})^{\mathrm{d}}\end{array}$ & 0.020 & 0.020 \\
\hline $\begin{array}{l}\text { Si detector cosine } \\
\text { response }\end{array}$ & 0.000 & 0.500 & $\begin{array}{l}\text { Si detector cosine } \\
\text { response }\end{array}$ & 0.000 & 1.500 \\
\hline $\begin{array}{l}\text { Pyrheliometer detector } \\
\text { temperature response } \\
\left(20^{\circ} \mathrm{C}\right)\end{array}$ & 0.250 & 0.050 & $\begin{array}{l}\text { Detector temperature } \\
\text { response }\end{array}$ & 3.000 & 0.050 \\
\hline $\begin{array}{l}\text { Pyrheliometer detector } \\
\text { linearity }\end{array}$ & 0.100 & 0.100 & $\begin{array}{l}\text { Day-to-day temperature } \\
\text { bias }\left(10^{\circ} \mathrm{C}\right)\end{array}$ & 0.125 & 0.100 \\
\hline $\begin{array}{l}\text { Solar alignment variations } \\
\text { (tracker or shade band) } \\
\text { and pyranometer level for } \\
\text { Si }\end{array}$ & 0.200 & 0.100 & $\begin{array}{l}\text { Solar alignment variations } \\
\text { (tracker or shade band) } \\
\text { and pyranometer level for } \\
\text { Si }\end{array}$ & 0.200 & 0.200 \\
\hline $\begin{array}{l}\text { Pyrheliometer window } \\
\text { spectral transmittance }\end{array}$ & 0.100 & 1.000 & $\begin{array}{l}\text { Pyrheliometer window } \\
\text { spectral transmittance }\end{array}$ & 0.500 & 1.000 \\
\hline $\begin{array}{l}\text { Optical cleanliness } \\
\text { (blockage) }\end{array}$ & 0.200 & 0.100 & $\begin{array}{l}\text { Optical cleanliness } \\
\text { (blockage) }\end{array}$ & 0.250 & 0.100 \\
\hline $\begin{array}{l}\text { Electromagnetic } \\
\text { interference and } \\
\text { electromagnetic field }\end{array}$ & 0.005 & 0.005 & $\begin{array}{l}\text { Electromagnetic } \\
\text { interference and } \\
\text { electromagnetic field }\end{array}$ & 0.005 & 0.005 \\
\hline
\end{tabular}

${ }^{\mathrm{a}}$ Thermopile detector used for a pyrheliometer.

${ }^{\mathrm{b}}$ Silicon diode sensor-based RSI.

${ }^{\mathrm{c}}$ Fossilized indicates that the calibration uncertainty is always carried forward into the field.

${ }^{\mathrm{d}}$ Typical manufacturer specified accuracy: $\pm 0.05 \%$ of full-scale range (typically $50 \mathrm{mV}$ ) $-25^{\circ}-50^{\circ}$ $\mathrm{C}$; assume $10-\mathrm{mV}$ signal so $\pm 50 \mu \mathrm{V}(0.5 \%)$ with $1.67 \mu \mathrm{V}$ resolution $(0.02 \%)$.

The uncertainty of the calibration is the most important contribution to the overall uncertainty for well-maintained high-quality instruments. The calibration stability of commercially available pyranometers and pyrheliometers is generally less than a $1 \%$ and $2 \%$ change in responsivity (Rs) per year, respectively. When finally deployed in the field, factors such as accuracy of solar tracking and/or leveling, data logger accuracy, cleanliness of the windows, and frequency of recalibration may contribute more sources of uncertainty, thus resulting in expanded uncertainties of $\pm 2.0 \%- \pm 2.5 \%$ in field DNI measurements and $\pm 3.0 \%- \pm 5 \%$ in field GHI measurements from a very carefully conducted, high-quality measurement system (Reda 2011).

The typical calibration uncertainty for any thermopile pyranometer with respect to a WRR reference cavity radiometer is $\approx 0.5 \%$ at any very narrow range $\left( \pm 2^{\circ}- \pm 5^{\circ}\right)$ of SZA (Reda et al. 2008). Typically, Rs is selected as an average responsivity for a specified SZA (usually $45^{\circ}$ ). However, the irradiance is collected for a wide range of SZAs $\left(0^{\circ}-85^{\circ}\right)$, and the measurement uncertainty over the whole range is larger. As shown in Chapter 3, for some pyranometers, Rs can vary by $\pm 3 \%- \pm 10 \%$ or even more over this zenith angle interval. These effects then need to be combined with the field measurement influences, the same as with the DNI measurement 
uncertainty estimate (e.g., including pyranometer installation, data logger accuracy, cleanliness, spectral dependency, or temperature sensitivity).

If only one Rs is used for a wide range of SZAs, that value is often derived for relatively low angles. Also, the variation of responsivity with SZA and azimuth angles is typically greater for high SZAs. Hence, large uncertainties usually occur at high SZAs. These high-zenith-angle-related uncertainties occur throughout parts of the day (morning and afternoon) when the available solar resource is much smaller than typical mid-day values and/or when SZAs are smaller. Because the minimum SZAs vary throughout the year, the uncertainty in hemispherical radiation data will vary as well.

Even in the good measurement regime of mid-day under clear-sky conditions, the uncertainty of hemispherical field measurement is typically two to three times that of direct-beam measurements, or $\pm 4 \%- \pm 5 \%$ throughout a year, primarily because of seasonal variations in uncertainty. Better instrumentation design and careful applications of correction factors as a function of SZA are ways to improve (reduce) the uncertainty in GHI measurements. The alternative is to use high-quality DNI and DHI measurements using a tracking shading disk/ball to compute GHI from the closure equation. The expanded uncertainties for this calculated GHI then approach that of DNI $( \pm 2 \%)$ for clear-sky measurements.

Figure 6-2 shows the calibration traceability for pyrheliometers used to measure DNI and for pyranometers used to measure GHI or DHI. The figure indicates how uncertainties accumulate from calibration to field deployment. Broad arrow boxes show the accumulated expanded uncertainty at each phase of the process. The resulting uncertainty in field deployment for pyrheliometers is $\pm 2.0 \%$, assuming regular and high-quality maintenance. Measurement uncertainties for pyranometers used to measure $\mathrm{GHI}$ in the field range from $\pm 3.0 \%$ for SZAs between $30^{\circ}$ and $60^{\circ}$ and up to $\pm 7 \%- \pm 10 \%$ for angles greater than $60^{\circ}$, again assuming regular and high-quality maintenance.

There is currently no specific method to calibrate pyranometers in a tilted position (i.e., for the measurement of GTI). They are thus calibrated on the horizontal, hence for the GHI measurement, before deployment. Tilting a pyranometer typically alters its responsivity due to, for example, changes in convection patterns inside the dome or changes in thermal offset. This typically affects the calibration uncertainty of GTI measurements. This caveat also holds for the measurement of upwelling irradiance using a down-facing pyranometer. (This measurement is necessary to obtain the surface albedo by dividing it by the GHI.)

The calibration and assessment of calibration and field uncertainties for pyrheliometers and pyranometers are described in detail in national and international standards (ASTM G167-05, ASTM E816-05, ASTM E824-05, ASTM G183-05, ISO 9059, ISO 9846, and ISO 9847). 


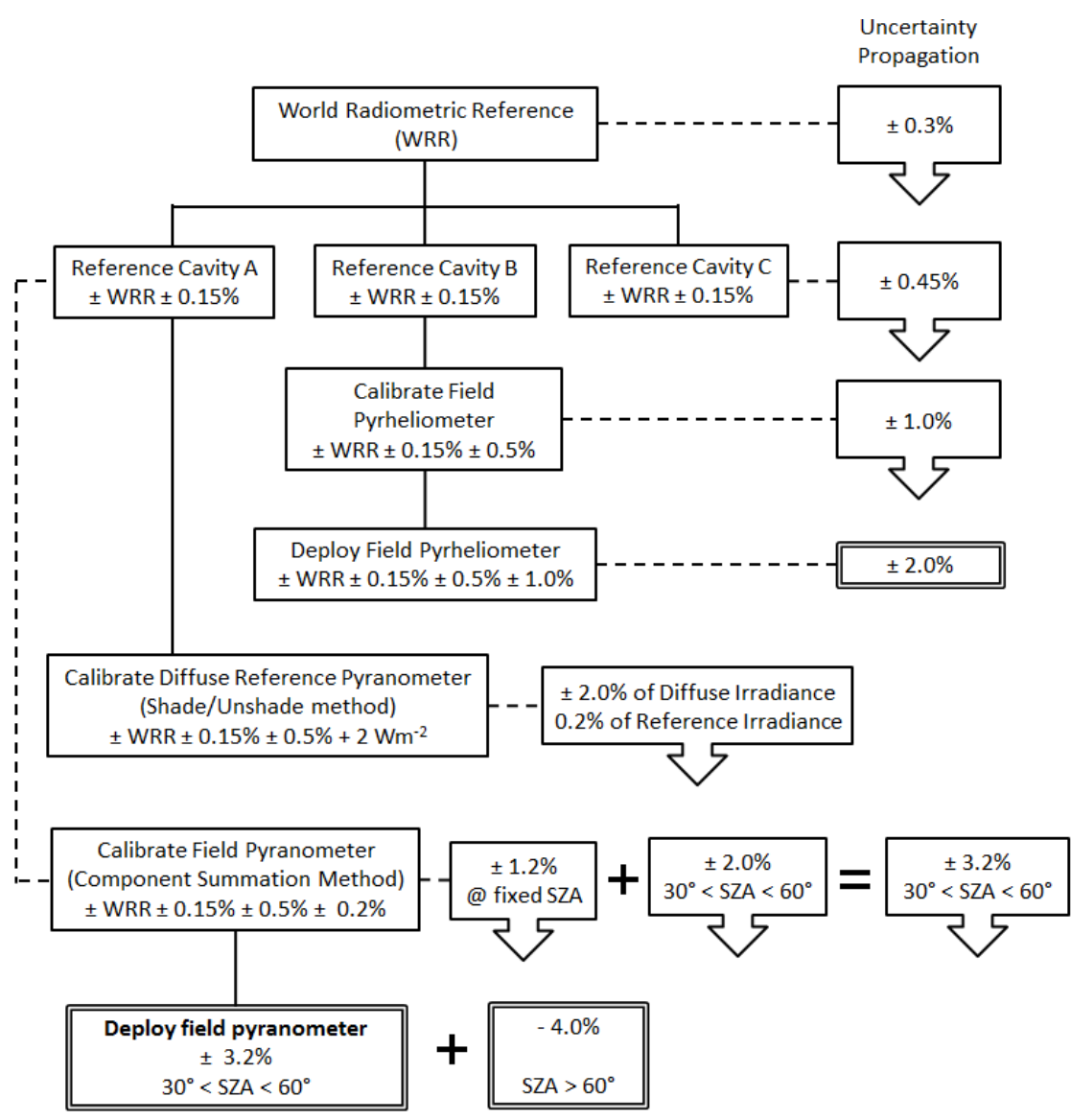

Figure 6-2. Calibration traceability and accumulation of measurement uncertainty for pyrheliometers and pyranometers (coverage factor $k=2$ ). Image by NREL

\subsection{Uncertainty Quantification of Solar Resource Estimates}

Solar radiation can be modeled in many different ways, depending on the available inputs, origin (ground-based or satellite-based), application requirements (e.g., clear-sky or all-sky conditions), and degree of detail (broadband or spectral irradiance).

Satellite-based models estimating solar radiation have used a physics-based approach using radiative transfer modeling, a semi-empirical approach relating the reflected radiance sensed by the satellite sensor directly to surface radiation, or a mix of both.

Models derived using semi-empirical correlations between ground-based irradiance measurements and reflected radiance observations from satellite sensors inherently carry the uncertainty of all these measurements. This uncertainty is embedded in the ultimate model accuracy, along with the uncertainties associated with the satellite sensors and the modeling process. Models based on $2 \%, 5 \%$, or $10 \%$ accurate ground-based irradiance measurements can be no more accurate than the data used to derive and/or validate the model. Similarly, models based on first principles of physics and radiation transfer cannot be validated or verified to a level of accuracy greater than that of the ground-based irradiance measurements. A thoroughly documented uncertainty analysis (Gueymard and Myers 2008b, 2009) is necessary to ascertain the validity of model accuracy claims. 
An understanding of the difference between the perspectives of satellite-derived irradiance values and ground-based measurements is essential when ground-based data are used to derive and validate satellite-derived irradiance values. Observations of a specific pixel (or grid cell) by a spaceborne radiometer ultimately provide (after substantial modeling) an estimate of surface radiation based on the estimated properties of those clouds and other atmospheric constituents spread throughout that pixel or a larger area. In contrast, surface irradiance observations are made by an instrument viewing the sky from a specific point. If the satellite pixel size is small enough, parallax errors enter into the comparison. Conversely, if it is too large, the radiation field over the pixel might not be homogenous enough for a correct comparison. Terrain effects may also influence a comparison in which cloudiness, elevation, and/or topographic shading may vary within a short distance.

\subsection{Historical Uncertainty Quantification Approach of Solar Resource Estimates from Models}

This section begins with a presentation of the historical uncertainty quantification approach of radiation models. This provides an overview of the progress in satellite-based data quality. Although there is no standardized method for accuracy assessment, most authors of the literature have usually reported the root mean square deviation and bias (absolute or relative). As an example, the model of Darnell et al. (1988) was used to evaluate surface radiation using cloud information from the ISCCP-C1 cloud database. The results were then compared to surface observations collected by the World Radiation Data Center (WRDC) by Darnell and Staylor (1992). The root mean square error (RMSE) from this comparison was found to be $\approx 16 \mathrm{Wm}^{-2}$, and the bias was $\approx 4 \mathrm{Wm}^{-2}$. Note that the interpretation of reported errors is dependent on the spatial and temporal resolution of the data being compared (random errors tend to decrease rapidly with increasing averaging period) and that the relative errors in modeled DNI are always greater than in $\mathrm{GHI}$ - opposite to what occurs with high-quality measurements.

According to Perez et al. (1987), satellite-based retrievals of DNI were accurate to $10 \%-12 \%$. According to Renné et al. (1999) and Zelenka et al. (1999), the target-specific comparison to ground-based observations had a relative RMSE of at least 20\%; the time-specific pixel-wide accuracy was $10 \%-12 \%$ on an hourly basis. Note that some publications report accuracy results in absolute unit (e.g., $\mathrm{Wm}^{-2}$ ), whereas others use a relative scale (percent), which complicates comparisons.

\subsection{Current Uncertainty Quantification Approach of Solar Resource Estimates from Models}

To improve modeled data integrity, a comprehensive representation of the model uncertainty method must be followed. The assessment of model uncertainty now attempts to replicate the developments made for measurement uncertainty, as detailed in Section 6.1. It is essential to use measurements of solar radiation made at ground stations from regions in various climates (or even microclimates) with the goal to perform a detailed evaluation of the modeled data set. However, measurements of solar radiation made at ground stations are temporally and spatially scarce, and they are expensive to maintain and quality control. Further, in order to perform an accurate evaluation of the model's predictions, it is critical that these ground-based irradiance measurements be of high quality and rely on low-uncertainty radiometers that follow the best practices for the collection, operation, maintenance, and quality assurance. 
Recent studies, such as those by Habte et al. (2017), Šúri and Cebecauer (2014), Wilcox (2012), or Cebecauer et al. (2011a), discussed quantification methods aimed at a comprehensive representation of the model uncertainty using the GUM method. This implements the error statistics (bias, RMSE, and uncertainty) of those ground-based irradiance measurements used to evaluate the modeled data. Knowledge of the latter's uncertainty is essential because it must be added to the model uncertainty. Equation 6-7 includes these sources and provides the uncertainty estimate for a $95 \%$ confidence interval, representing two standard deviations (coverage factor of $\sim 2)$.

$$
U_{95}=k * \sqrt{\left(\frac{U_{\text {meas }}}{k}\right)^{2}+\left(\frac{\text { bias }}{k}\right)^{2}+\left(\frac{R M S E}{k}\right)^{2}}
$$

where $U_{\text {meas }}$ is the estimated uncertainty in ground-based irradiance measurements ("ground truth"), and both bias and RMSE are derived from the model's validation analyses. As described in Section 6.1.1, these three statistics are divided by $k(\approx 2$ because a normal distribution is assumed).

Habte et al. (2017) determined the overall uncertainty of the modeled irradiance data in the National Solar Radiation Database (NSRDB 1998-2015). This estimation was made using hourly averages, daily totals, monthly means of daily totals (MMDT), and annual differences (Figure 6-3). Varying the time interval helps capture the temporal uncertainty of the specific modeled solar resource data required for each phase of a solar energy project. For instance, the annual data uncertainty estimate is important for financial analysts during the conceptual phase of a project, whereas the uncertainty in hourly data is essential during the engineering design phase and due-diligence studies. 


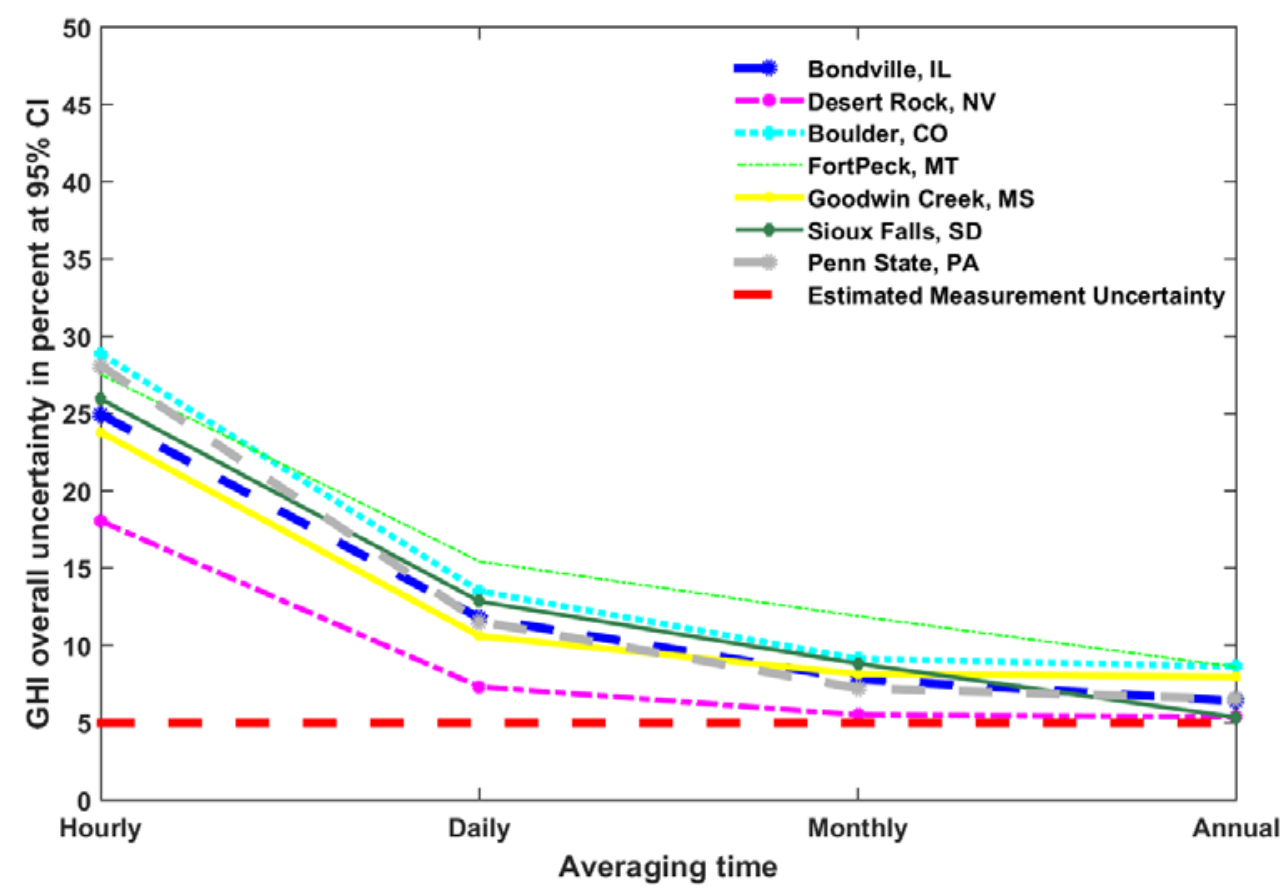

Figure 6-3. Uncertainty estimation of the modeled GHI in the NSRDB (1998-2015) for the 95\% confidence interval $(\mathrm{Cl} ; k \approx 2)$ under various time averages at seven National Oceanic and Atmospheric Administration (NOAA)SURFace RADiation (SURFRAD) locations

As shown in Figure 6-3, an uncertainty of 5\% was chosen for the measurements and kept constant throughout the averaging time because the main uncertainty of radiometers (due to calibration) did not change with averaging time (Habte et al. 2017; Reda 2011). The relative bias and RMSE associated with Figure 6-3 are shown in Figure 6-4 (left and right, respectively).
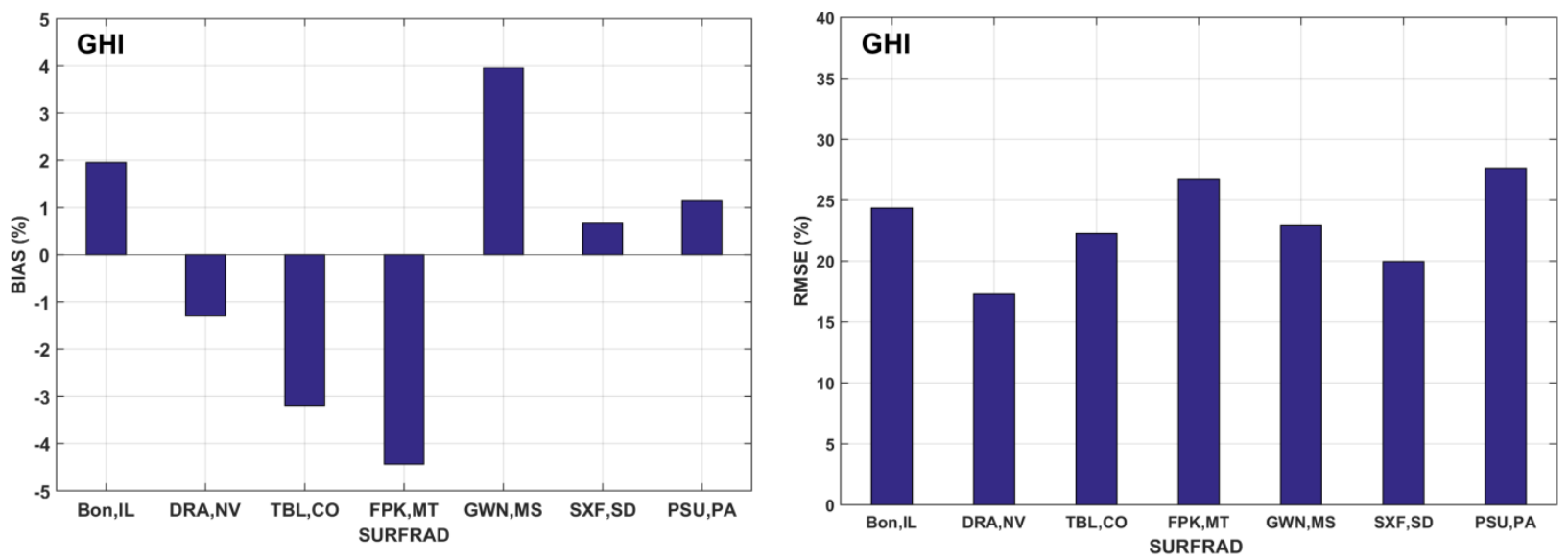

Figure 6-4. Relative bias (left) and RMSE (right); comparison results of modeled data from the NSRDB (1998-2015) relative to irradiance measurements made at seven stations from the NOAA SURFRAD network

Solargis implemented a similar approach to determine uncertainty in their satellite-derived data sets by incorporating the model uncertainty (bias and RMSE), the uncertainty of the ground- 
based irradiance measurements, and the inter-annual irradiance variability (Equation 6-8). The annual solar resource is thus allowed to vary from the long-term averages (see, e.g., Figure 6-5). A detailed discussion about the relative importance of these uncertainties is provided in Cebecauer et al.

(2011a). $u_{\text {combined }}= \pm \sqrt{\left(U_{\text {meas }}\right)^{2}+(\text { bias })^{2}+(R M S E)^{2}+\left(U_{\text {interannual varibility }}\right)^{2}}$

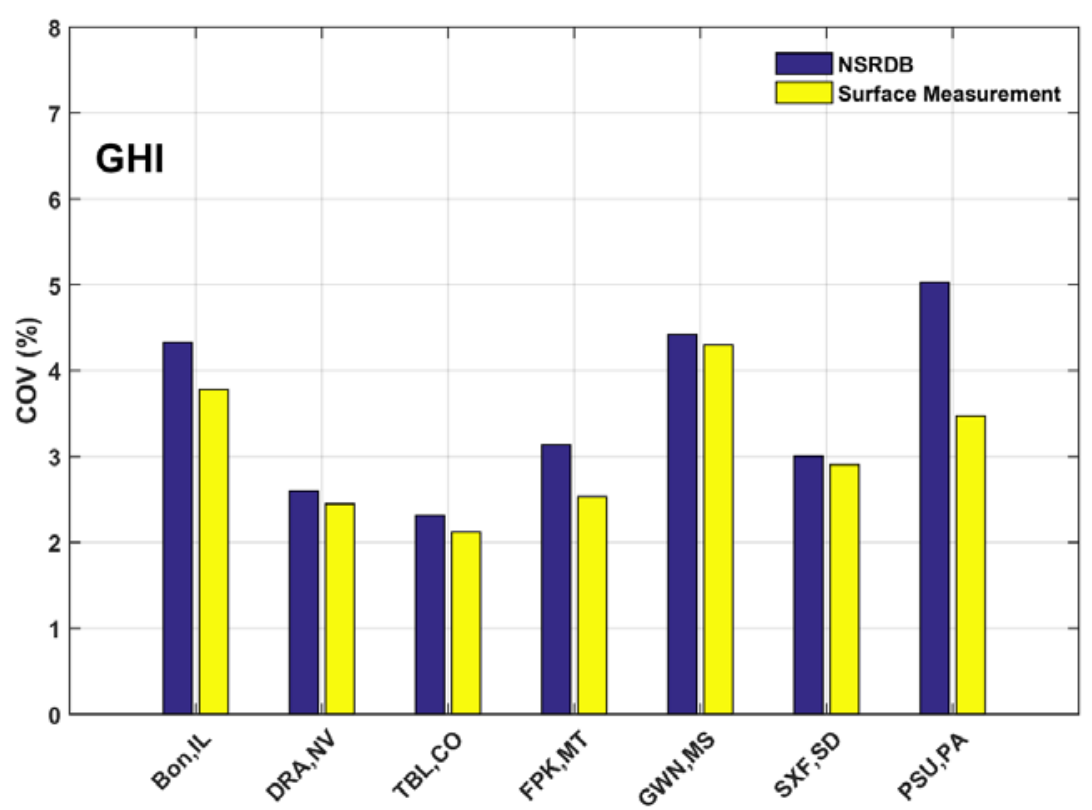

Figure 6-5. Inter-annual variability in modeled NSRDB data (1998-2015) and in ground-based irradiance measurements at seven SURFRAD sites using the coefficient of variation (COV) metric

Habte et al. (2017) calculated an inter-annual variability metric using Equations 6-9 and 6-10.

$$
\begin{aligned}
& S D=\sqrt{\left(\frac{1}{n} \sum_{i=1}^{n}\left(a_{n}-\hat{\mathrm{a}}\right)^{2}\right)} \\
& U_{\text {inter-annual varibility }(\%)}=\operatorname{COV}(\%)=\frac{\text { std }}{\hat{\mathrm{a}}} * 100
\end{aligned}
$$

where $S D$ is the standard deviation, and $a_{n}$ is the irradiance of the individual $n$ years. The mean irradiance over 18 years (1998-2015) is shown as â.

In parallel, the accuracy of satellite-derived modeled data can be determined using various other statistical indicators, such as the mean absolute error (MAE) or the Kolmogorov-Smirnov (KS) test (Massey 1951). The KS test is a rigorous nonparametric method that is currently gaining acceptance for benchmarking satellite-retrieved GHI and DNI against ground-based observations (Espinar et al. 2009; Gueymard 2014). This test has the advantage of being nonparametric and is therefore not distribution dependent. It compares the two distributions of irradiance to evaluate their resemblance. 


\subsection{Challenges with Modeled Data Uncertainty Estimation}

Satellite-derived irradiance data sets have various embedded sources of uncertainty (Cebecauer et al. 2011a; Perez et al. 2013).

The satellite observations are used for the detection of clouds. The satellite pixel represents a certain area, typically $1-100 \mathrm{~km}^{2}$. Depending on that size, some subpixel variability and cloudinduced parallax effects may contribute to higher random errors in both GHI and DNI, as suggested by previous studies (e.g., Habte et al. 2017; Cebecauer et al. 2011a; Zelenka et al. 1999). The resolution of satellite images has limits to adequately describe properties of small and scattered clouds in the case of intermittent cloud situations. In tropical rainforest climates, it is often challenging to find cloudless situations for characterizing the reference surface albedo. At high latitudes, the low satellite viewing angles introduce errors in the detection of cloud position and properties (the satellite sensor most often sees clouds from the side rather than from the top). For intermittent cloud situations, the major part of the observed random errors (evaluated by RMSE statistics) is driven by inadequacies in the cloud-related parts of the radiative transfer algorithms.

Another important issue may be caused by aerosols. They tend to affect DNI 3-4 times more than GHI, depending on the relative proportions of absorption and scattering for the specific aerosol mixture of the moment and location. For example, mineral dust is mostly scattering, whereas black carbon is highly absorbing. At any instant, the aerosol optical depth (AOD) varies spectrally, so the common use of a single broadband AOD may result in additional uncertainties. Monthly average (or "climatological") AOD data are often used for resource assessment, but this may introduce significant errors in long-term DNI estimates (Ruiz-Arias et al. 2016). This is more likely to happen over areas of biomass burning, urban air pollution, and dust storms where aerosol climatology tends to smooth out episodic high-AOD events. Therefore, AOD data with daily resolution are advantageously used in some modeling approaches (Cebecauer et al. 2011b).

In regions with variable or complex landscape patterns (e.g., high spatial variability due to land/water mosaics, complex urbanization, or mountains), the surface reflectance properties change rapidly, both over space and time domain and even over distances that are shorter than the satellite's spatial resolution. Compared to neighboring rural or natural landscapes, large urban or industrial areas have much higher and temporarily changing concentrations of aerosols and water vapor. Over mountains, rapid changes in elevation also induce rapid changes in concentration of key atmospheric constituents and in cloud properties. In addition, 3D effects and terrain shading contribute to the complexity of conditions, which are to be approximated by the solar radiation models.

Another difficulty inherent to satellite-derived data sets is the poor discrimination between clouds and snow-covered surfaces when using the visible imagery only. This is because both situations have a high reflectance with visible imagery. Hence, a clear-sky scene over a snowy ground may look like an overcast sky, resulting in a strong overestimation or underestimation of both GHI and DNI, depending on the situation. One such example is known as the "Eugene syndrome" (Gueymard and Wilcox 2011). The use of multiple channels in the visible and infrared can solve this issue but requires more analysis and computer time. 
Finally, specular reflections, especially from sandy deserts or snowy/icy surfaces during certain times of the day, may result in the satellite image being interpreted as temporarily cloudy and, thus, be an underestimation of both GHI and DNI. This issue can be resolved by theoretically estimating the probability of specular reflection for such areas and factoring that into the calculation of surface radiation.

\subsubsection{Indicative Uncertainty of Modern Satellite-Based Models}

As an example, experience based on more than 200 validation sites shows that the state-of the-art semi-empirical satellite models can estimate the annual GHI with bias in the range of $\pm 4 \%$ when normalized to daytime irradiation (Cebecauer \& Š́ri 2012). This bias value depends on the geography. It can be higher (up to at least $\pm 8 \%$ ) in complex tropical regions; in areas with high atmospheric pollution, high latitudes, high mountains, and complex terrain; and in regions with low sun angles and occurrence of snow. Typical bias for DNI estimates at a specific site is about twice that of GHI.

The main sources of increased random errors are clouds, and to a lesser extent, changes in snow cover and increased dynamics of aerosols. Over arid and semi-arid areas or during sunny seasons, the RMSE of hourly GHI values is normally in the range of $7 \%-20 \%$. In more cloudy regions with more complex weather patterns, higher dynamics of atmospheric constituents, complex landscapes, or mid latitudes, the hourly RMSE increases to $15 \%-30 \%$. Over high mountains, high latitudes, or during seasons with low sun angles and frequent occurrences of snow, the relative RMSE for GHI can be $25 \%-35 \%$ or more. Similar patterns of RMSE can be observed also for the hourly DNI but with about twice the errors just mentioned for GHI. In arid and semi-arid zones, which are of the highest interest for concentrated solar energy technologies, RMSE in the range $18 \%-30 \%$ is typical. In more cloudy regions, with higher dynamics of aerosols, RMSE reaches $25 \%-45 \%$. Finally, at high latitudes and over mountains, RMSE may exceed $45 \%$. 


\section{References}

ASTM. 2005. E816-05: Standard Test Method for Calibration of Pyrheliometers by Comparison to Reference Pyrheliometers. West Conshohocken, PA: ASTM International.

ASTM. 2005. E824-05: Transfer of Calibration from Reference to Field Radiometers. West Conshohocken, PA: ASTM International.

ASTM. 2005. G167-05: Standard Test Method for Calibration of a Pyranometer Using a Pyrheliometer. West Conshohocken, PA: ASTM International; 21 pp.

ASTM. 2005. G183-05: Standard Practice for Field Use of Pyranometers, Pyrheliometers and UV Radiometers. West Conshohocken, PA: ASTM International.

ASTM. 2017. G213-17: Standard Guide for Evaluating Uncertainty in Calibration and Field Measurements of Broadband Irradiance with Pyranometers and Pyrheliometers. West Conshohocken, PA: ASTM International.

Cebecauer T., R. Perez, and M. Š́ri. 2011a. "Comparing Performance of Solargis and SUNY Satellite Models Using Monthly and Daily Aerosol Data." Proceedings of the ISES Solar World Congress 2011, Kassel, Germany.

Cebecauer, T., M. Suri, and C. A. Gueymard. 2011b. "Uncertainty Sources in Satellite-Derived Direct Normal Irradiance: How Can Prediction Accuracy Be Improved Globally?" Proceedings of the SolarPACES Conference. Accessed December 20, 2016.

http://www.solarconsultingservices.com/Cebecauer-Uncertainty $\% 20$ satell $\% 20$ dataSolarPACES11.pdf.

Darnell, W. L., and W. F. Staylor. 1992. "Seasonal Variation of Surface Radiation Budget Derived From International Satellite Cloud Climatology Project C1 Data.” Journal of Geophysical Research 97(15): 741-60.

Darnell, W. L., W. F. Staylor, S. K. Gupta, and M. Denn. 1988. "Estimation of Surface Insolation Using Sun-Synchronous Satellite Data.” Journal of Climate 1: 820-35.

Espinar, B., L. Ramirez, A. Drews, H. G. Beyer, L. F. Zarzalejo, J. Polo, and L. Martin. 2009. "Analysis of Different Error Parameters Applied to Solar Radiation Data from Satellite and German Radiometric Stations." Solar Energy 83(1): 118-25.

Finsterle, W. 2011. WMO International Pyrheliometer Comparison: IPC-XI 27 Sept.-15 Oct. 2010—WMO IOM No. 108. Davos, Switzerland: World Meteorological Organization.

Gueymard, C. A. 2014. A Review of Validation Methodologies and Statistical Performance Indicators for Modeled Solar Radiation Data: Towards a Better Bankability of Solar Projects. Renewable and Sustainable Energy Reviews 39: 1024-34.

Gueymard, C. A., and D. R. Myers. 2008a. "Solar Radiation Measurement: Progress in Radiometry for Improved Modeling." In: Modeling Solar radiation at the Earth Surface, V. Badescu (Ed.), Springer, pp. 1-27. 
Gueymard, C. A., and D. R. Myers. 2008b. "Validation and Ranking Methodologies for Solar Radiation Models." In: Modeling Solar Radiation at the Earth Surface, V. Badescu (Ed.), Springer, pp. 479-509.

Gueymard, C.A., and D. R. Myers. 2009. "Evaluation of Conventional and High-Performance Routine Solar Radiation Measurements for Improved Solar Resource, Climatological Trends, and Radiative Modeling." Solar Energy 83:: 171-85.

Gueymard, C. A., and S. M. Wilcox. 2011. "Assessment of Spatial and Temporal Variability in the U.S. Solar Resource From Radiometric Measurements and Predictions From Models Using Ground-Based or Satellite Data." Solar Energy 85: 1068-84..

Habte, A., M. Sengupta, I. Reda, A. Andreas, and J. Konings. 2014. "Calibration and Measurement Uncertainty Estimation of Radiometric Data: Preprint." Paper presented at Solar 2014, San Francisco, California, American Solar Energy Society.

Habte, A., M. Sengupta, A. Andreas, S. Wilcox, and T. Stoffel. 2016a. "Intercomparison of 51 Radiometers for Determining Global Horizontal Irradiance and Direct Normal Irradiance Measurements." Solar Energy 133: 372-93.

Habte, A., M. Sengupta, A. Andreas, I. Reda, and J. Robinson. 2016b. "The Impact of Indoor and Outdoor Radiometer Calibration on Solar Measurements." NREL/PO-5D00-66668. Golden, CO: National Renewable Energy Laboratory. http://www.nrel.gov/docs/fy17osti/66668.pdf.

Aron Habte, Manajit Sengupta, Anthony Lopez. 2017. Evaluation of the National Solar Radiation Database (NSRDB): 1998-2015. NREL/TP-5D00-67722. http://www.nrel.gov/docs/fy17osti/67722.pdf.

ISO. 1990. ISO 9059:1990: Calibration of Field Pyrheliometers by Comparison to a Reference Pyrheliometer. Geneva, Switzerland: ISO.

ISO. 1992. ISO 9847: 1992 (E): Solar Energy-Calibration of Field Pyranometers by Comparison to a Reference Pyranometer. Geneva, Switzerland: ISO; 20 pp.

ISO. 1993. ISO 9846: 1993(E): Solar Energy-Calibration of a Pyranometer Using a Pyrheliometer. Geneva, Switzerland: ISO; 24 pp.

ISO. 2008. ISO/IEC Guide 98-3:2008(E): Uncertainty of measurement - Part 3: Guide to the expression of uncertainty in measurement (GUM: 1995).Geneva, Switzerland: ISO, December 2008, $130 \mathrm{p}$.

Myers, D. R., T. L. Stoffel, S. Wilcox, I. Reda, and A. Andreas. "Recent Progress in Reducing the Uncertainty in and Improving Pyranometer Calibrations." Journal of Solar Energy Engineering 124: 44-50.

Perez, R., R. Seals, P. Ineichen, P. Stewart, and D. Menicucci. 1987. "A New Simplified Version of the Perez Diffuse Irradiance Model for Tilted Surfaces: Description Performance Validation.” Solar Energy 39: 221-32. 
Perez, R., T. Cebecauer, and M. Súri. 2013. "Semi-Empirical Satellite Models.” In: Kleissl J. (Ed.) Solar Energy Forecasting and Resource Assessment. Academic press.

Reda, I. 2011. Method to Calculate Uncertainties in Measuring Shortwave Solar Irradiance Using Thermopile and Semiconductor Solar Radiometers. NREL/TP-3B10-52194. Golden, CO: National Renewable Energy Laboratory. http://www.nrel.gov/docs/fy11osti/52194.pdf.

Reda, I., D. Myers, and T. Stoffel. 2008. "Uncertainty Estimate for the Outdoor Calibration of Solar Pyranometers: A Metrologist Perspective.” Measure 3(4): 58-66. NREL/JA-581-41370.

Reda, I., M. Dooraghi, and A. Habte. 2013. NREL Pyrheliometer Comparison: September 16 to 27, 2013 (NPC-2013). NREL/TP-3B10-60749. Golden, CO: National Renewable Energy Laboratory.

Renné, D. S., R. Perez, A. Zelenka, C. Whitlock, and R. DiPasquale. 1999. Advances in Solar Energy: An Annual Review of Research and Development. Vol. 13. Goswami, D. Y., and Boer, K.W., Eds. Boulder, CO: American Solar Energy Society.

Ruiz-Arias, J.A., C. A. Gueymard, F. J. Santos-Alamillos, and D. Pozo-Vázquez. 2016. "Worldwide Impact of Aerosol's Time Scale on the Predicted Long-Term Concentrating Solar Power Potential. Nature Scientific Reports 6: 30546. doi:10.1038/srep30546

Stoffel, T. L., I. Reda, D. R. Myers, D. Renné, S. Wilcox, and J. Treadwell. 2000. “Current Issues in Terrestrial Solar Radiation Instrumentation for Energy, Climate, and Space Applications." Metrologia 37(5): 399-401.

Š́ri, M., and T. Cebecauer. 2014. "Satellite-Based Solar Resource Data: Model Validation Statistics Versus User's Uncertainty." Proceedings of the 43th ASES National Solar Conference.

Vuilleumier, L., M. Hauser, C. Flix, F. Vignola, P. Blanc, A. Kazantzidis, and B. Calpini. 2014. "Accuracy of Ground Surface Broadband Shortwave Radiation Monitoring." Journal of Geophysical Research Atmospheres, 119(24): 838- 860.

Wilbert, Stefan, Stefan Kleindiek, Bijan Nouri, Norbert Geuder, Aron Habte, Marko Schwandt, and Frank Vignola. 2016. "Uncertainty of Rotating Shadowband Irradiometers and SiPyranometers Including the Spectral Irradiance Error.” AIP Conference Proceedings no. 1734 (1):150009. doi: http://dx.doi.org/10.1063/1.4949241

Wilcox, S. M. 2012. National Solar Radiation Database 1991-2010 Update: User's Manual. NREL/TP-5500-54824. Golden, CO: National Renewable Energy Laboratory. http:/www.nrel.gov/docs/fy12osti/54824.pdf.

Wilcox, S.M., and D. Myers. 2008. Evaluation of Radiometers in Full-Time Use at the National Renewable Energy Laboratory Solar Radiation Research Laboratory NREL/TP-550-44627. Golden, CO: National Renewable Energy Laboratory. http://www.nrel.gov/docs/fy09osti/44627.pdf. 
Zelenka, A., R. Perez, R. Seals, and Renné, D. 1999. "Effective Accuracy of Satellite-Derived Irradiance." Theoretical and Applied Climatology 62: 199-207. 


\title{
7 Forecasting Solar Radiation
}

\author{
Elke Lorenz, ${ }^{1}$ Jose Antonio Ruiz-Arias, ${ }^{2}$ and Stefan Wilbert ${ }^{3}$ \\ 1. Fraunhofer ISE \\ 2. Solargis \\ 3. German Aerospace Center (DLR)
}

\subsection{Introduction}

Solar resource forecasting is very important for the operation and management of solar power plants. Solar radiation is highly variable because it is driven mainly by synoptic and local weather patterns. This high variability presents challenges to meeting power production and demand curves, notably in the case of photovoltaic (PV) power plants, which have little or no storage capacity. For concentrating solar power (CSP) plants, variability issues are partially mitigated by the thermal inertia of the plant, including its heat transfer fluid, heat exchangers, turbines and, potentially, coupling with a heat storage facility; however, temporally and spatially varying irradiance introduces thermal stress in critical system components and plant management issues that can result in the degradation of the overall system's performance and reduction of the plant's lifetime. The variability can also result in lower plant efficiencies compared to operation in stable conditions because optimally operating the plant is more challenging. For PV power plants that have battery storage, forecasts are helpful to schedule the charging process of the batteries at the most appropriate time, optimize the fractions of electricity delivered and stored at any instant, and thus avoid the loss of usable energy.

Solar radiation forecasting anticipates the solar radiation transients and the power production of solar energy systems, allowing for the setup of contingency mechanisms to mitigate any deviation from the required production. With the expected integration of large shares of solar power, reliable predictions of solar power production are becoming increasingly important as a basis for efficient management and operation strategies as well as for solar energy trading.

Today, solar power prediction systems are an essential part of electric grid management in countries that have substantial shares of solar power generation, among which Germany is a paradigmatic case. For example, in 2016 Germany had an installed PV power capacity of more than $40 \mathrm{GW}_{\text {peak, }}$, supplying more than $40 \%$ of the total load on sunny summer days at noon. In this context, and according to the German Renewable Energy Sources Act (a set of laws aimed at promoting renewable energies in Germany), transmission system operators are in charge of marketing and balancing the overall fluctuating PV power feed-in, which enforces the use of regional forecasts for the designated control areas. Additionally, there is optional direct marketing of PV power based on forecasts for the respective PV power plants' output. PV power is first offered on the day-ahead auction at the European Power Exchange. Subsequently, amendments based on updated forecasts can be made on the intraday market, when electricity might be traded until 45 minutes before delivery begins. Remaining deviations between scheduled and needed power are adjusted using balancing power. A similar procedure for California's electricity market is described in Mathiesen, Kleissl, and Collier (2013). Also, Kleissl (2013) describes the stakeholder needs from the perspective of independent system operators and energy traders. Hence, accurate PV power forecasts at different spatial and temporal scales are very important for cost-efficient grid integration because large errors in the day-ahead forecast can cause either very high or negative prices on the intraday market and intraday forecast errors determine the need for costly balancing power. 
Several studies have evaluated the added value of solar irradiance forecasting for solar energy applications. For example, Dumortier (2009) give a preliminary overview of such applications. Many other authors have described in detail specific use cases and benefits of solar power forecasting; following is a nonexhaustive list of some of these. In the realm of electric grids, Perez et al. (2007) evaluated the operational accuracy of end-use forecasts and their ability to predict the effective capacity of grid-connected PV power plants. Kaur et al. (2016) described the benefits of solar forecasting for energy imbalance markets. Rikos et al. (2008), Diagné et al. (2013), and Simoglou et al. (2014) examined the solar power forecasting requirements to support microgrid and island systems with respect to stability and power quality. More specifically, Martinez-Anido et al. (2016) evaluated the value of solar forecast improvements for the Independent System Operator New England. At the power plant level, Marcos et al. (2013) describe the benefits of power prediction to optimize a storage system that attenuates the power fluctuations in large PV power plants. Almeida, Perpiñan, and Narvarte (2015) have explored the skill of a nonparametric method to predict the AC power output of PV power plants.

Regarding CSP and direct normal irradiance (DNI), Wittmann et al. (2008) and Kraas, Schroedter-Homscheidt, and Madlener et al. (2013) use case studies to show the economic benefit of supplying DNI forecasts for optimized operation strategies of CSP plants. Schroedter-Homscheidt et al. (2013) evaluated the aerosol forecasting requirements for forecasts of concentrating solar electricity production. Law et al. (2014) reviewed different DNI forecasting methods and their applications for yield forecasting of CSP plants. In a later work, Law, Kay, and Taylor (2016) reviewed the benefits of short-term DNI forecasts for the CSP technology. Hirsch et al. (2014) specifically evaluated the use of $6 \mathrm{~h}$ forecasts (nowcasting) to operate CSP plants.

In a broader context, different solar radiation forecasting approaches-targeted at various time horizons-have been developed using different input data and data processing methods. A nonexhaustive list includes methods based on statistical inference on ground-observed time series (Huang et al. 2013; Lonij et al. 2013; Voyant et al. 2014; Boland and Soubdhan 2015; Graditi, Ferlito, and Adinolfi 2016), use of cloud motion vectors and other cloud advection techniques on all-sky cameras and satellite imagery (Hammer et al. 1999; Perez et al. 2010; Chow et al. 2011; Marquez and Coimbra 2013; Quesada-Ruiz et al. 2014; Schmidt et al. 2016; Lee et al. 2017; Arbizu-Barrena et al. 2017), forecasts based on numerical weather prediction (NWP) models (Mathiesen and Kleissl 2011; Lara-Fanego et al. 2012; Pelland, Galanis, and Kallos 2013; Ohtake et al. 2013 Perez et al. 2013; Jimenez et al. 2016a; Jimenez et al. 2016b) or even hybrid techniques (Marquez and Coimbra 2011; Marquez, Pedro, and Coimbra 2013; Perez et al. 2014; Dambreville et al. 2014; Wolff et al. 2016; Mazorra Aguiar et al. 2016). All these methods are explained in Section 7.2. Comprehensive general overviews can be found in Inman et al. (2013) and Antonanzas et al. (2016).

In this chapter, we provide an overview of basic concepts of solar irradiance forecasting by referring to selected examples and operational models rather than reviewing the state of the art - which can be found elsewhere, such as in Lorenz and Heinemann (2012); Inman et al. (2013); Kleissl, Schroedter-Homscheidt, and Madlener (2013); and, for PV applications, Antonanzas et al. (2016). The evaluations and comparisons of different irradiance forecasting approaches focus on global horizontal irradiance (GHI), and DNI is discussed in less detail. Nonetheless, forecasting and, in particular, evaluation methods apply to DNI to some extent. A focus on DNI forecasting can be found in Schroedter-Homscheidt and Wilbert (2017). 
Although irradiance is a key driver for solar power output, other environmental factors have a nonnegligible impact on the final power yield of the plant, such as ambient temperature, air humidity, wind speed, and wind direction. Ambient temperature and humidity, for instance, affect the PV efficiency and the thermal regime of CSP plants. Similarly, wind speed forecasting - and especially wind gust forecasting - is important to prevent strong mechanical loads in tracking systems. Therefore, the forecast of these other ancillary factors will also result in tangible benefits for the effective operation of power plants; however, they are not discussed here.

\subsection{Solar Irradiance Forecasting Methods}

Depending on the specific application and requirements with respect to forecast horizon and spatiotemporal resolution, different forecasting methods are customarily used. From short to long forecasting horizons, the most important solar forecasting methods are the following (see also Figure 7-1):

- Intra-hour forecasts with high spatial and temporal resolution. They require on-site observations of irradiance and/or cloud conditions that are processed using statistical methods and also, more recently, artificial intelligence and machine learning models. Those that are based on solar irradiance measurements and, for instance, conventional auto-regressive techniques might provide meaningful forecasts even up to a few hours ahead under stable sky conditions; however, they are rarely predictable under variable sky conditions given the chaotic behavior of the cloud system and the limited information held in point-wise observations. In these cases, the local distribution of clouds as gathered by one or more ground-based sky imagers might enhance the forecast reliability. This cloud-related information allows producing solar irradiance forecasts with temporal resolution on the order of a few minutes and spatial resolution within the range from 10$100 \mathrm{~m}$ in the form of maps covering a few square kilometers around the sky imager(s). The typical forecast horizon of these systems is 10-20 min, depending on the cloud variability and typology.

- Forecasts from 4-6 h ahead. These are sometimes referred to as nowcasts, and they are conventionally derived by extrapolating the cloud locations into the future using cloud motion vector (CMV) techniques on satellite images (see Section 7.2.2). The typical spatial resolution is from $1-5 \mathrm{~km}$ for the current generation of geostationary satellites, with forecast updates every 10-30 min.

- Intra-day and day-ahead forecasts. These are based on NWP models, which typically offer higher performance for forecast horizons more than approximately $4-6 \mathrm{~h}$ up to several days ahead. These models predict the evolution of the atmospheric system, including the formation, advection, diffusion, and dissipation of clouds based on a physical description of the dynamic processes occurring in the atmosphere and solving the system of equations that governs these processes from an observed set of initial conditions. Current global NWP models cover the Earth with spatial resolutions ranging from approximately $0.1-0.5^{\circ}$ and temporal resolutions from $1-3 \mathrm{~h}$. Regional models, which are sometimes referred to as limited area models or mesoscale models, might be subsequently used to further downscale the forecasts delivered by global models to spatial resolutions of very few kilometers and intra-hourly temporal resolutions in the area of interest.

In addition to this broad classification, when historical or near-real-time on-site solar irradiance or PV yield observations are available, these methods—and, very particularly, 
satellite-based and NWP-based methods-can be further improved by using model output statistics (MOS) techniques, sometimes also referred to as statistical downscaling techniques (whereas NWP models are known as dynamical downscaling techniques). MOS-based methods learn error patterns by comparing forecasts and observations and using them to reduce the error of the final prediction.

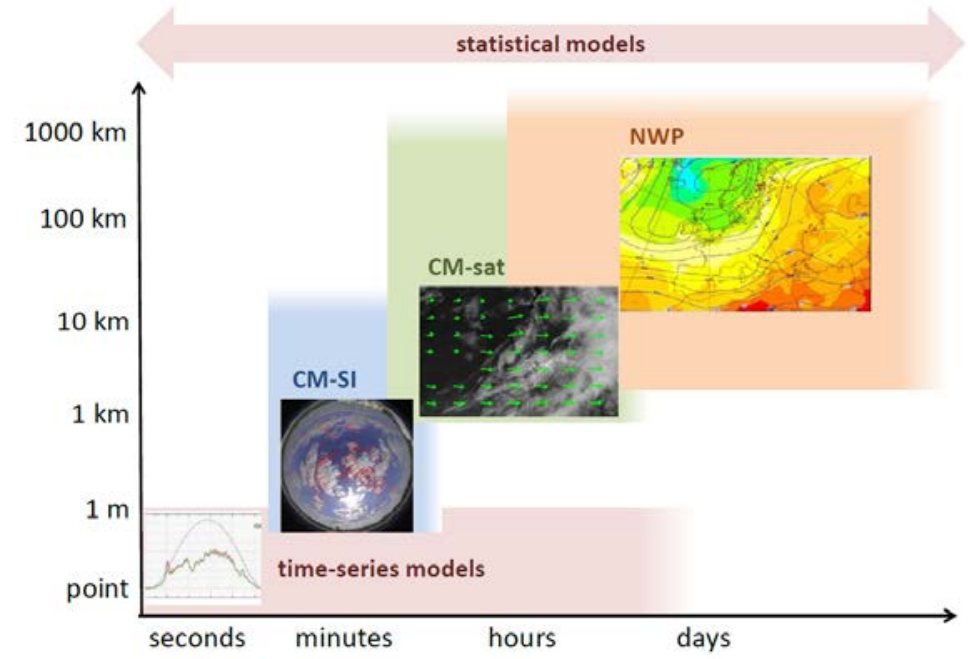

Figure 7-1. Illustration of different forecasting methods for various spatial and temporal scales. The $y$-axis shows the spatial resolution, and the $x$-axis shows the forecast horizon intended for the different forecasting techniques. CM-SI: cloud motion forecast based on sky imagers; CMsat: cloud motion forecast based on satellite images. Statistical models apply to all forecast horizons.

\subsubsection{On-Site Observations and Statistical Forecasting Models}

Statistical learning models are widely used for solar irradiance and power forecasting. The dependence between input variables (predictors) and forecast values (predictands) is established in a training phase by learning from historical data, assuming that patterns in the historical data sets are repeated in the future and thus might be exploited for forecasting. Statistical methods include classical regression methods, such as autoregressive and autoregressive integrated moving average models; as well as artificial intelligence techniques, such as artificial neural networks, k-nearest neighbors, or support vector regression. Coimbra and Pedro (2013) and Diagné et al. (2013) provide an overview of different statistical approaches used for solar irradiance forecasting. Here, we refer mainly to selected examples investigated in the International Energy Agency (IEA) Solar Heating and Cooling Programme (SHC) Task 36 and Task 46.

Intra-hour or hours-ahead solar irradiance and PV power forecasting with time series models use recent measurements of irradiance or PV power as a basic input, possibly complemented by measurements of other variables. Examples are the application of a coupled autoregressive and dynamic system model for forecasting solar radiation on an hourly timescale as described by Huang et al. (2013), the comparison of artificial neural networks and classical time series models as in Reikard (2009), and the short-term PV power prediction approach of Bacher, Madsen, and Nielsen (2009).

For any statistical model, the selection and availability of appropriate input variables as well as the optimized preprocessing of these data is of critical importance for good forecast performance. Also, the choice of the model configuration (e.g., the artificial neural network architecture or the selection of hyper-parameters in machine learning models) is essential. 
Finally, the setup of the training sample (e.g., the number of days and sites used for the training) has a remarkable influence on the forecast accuracy. Coimbra and Pedro (2013) showed the benefits of the application of a genetic algorithm to identify the most suitable artificial neural network architecture, preprocessing scheme, and training data. In the following, we briefly discuss advantages and limits of purely statistical approaches.

Statistical models exploit the autocorrelation in time series of solar irradiance, cloud cover and, possibly, other explanatory variables. Given the inherent chaotic nature of weather phenomena, any existing autocorrelation shrinks as the time lag between time series instances increases. Hence, the performance of these models is strongly determined by (1) the underlying autocorrelation of each particular weather condition and (2) decreases as forecast lead time increases. For forecast horizons up to $2 \mathrm{~h}$ ahead-possibly longer for relatively stable sky conditions - forecasts based on accurate on-site measurements and statistical methods are advantageous compared to other approaches. For longer forecast horizons, the autocorrelation becomes so small that wide-area observations (such as those from satellites) or physically-based methods (such as NWP models) are required to meet the forecast skill requirements. In this context, Bacher, Madsen, and Nielsen (2009) performed a comparison of an autoregressive model for hourly solar power forecasting combined with and without exogenous inputs from a diverse origin. They found that ground-observed data are the most important class of inputs up to approximately $2 \mathrm{~h}$ ahead, whereas the NWP forecast parameters are adequate for next-day horizons; however, these conclusions highly depend on the prevailing climate and weather conditions.

\subsubsection{Irradiance Forecasting with Cloud Motion Vectors}

In timescales of a few hours, the temporal evolution of cloud patterns is sometimes dominated by horizontal advection, with the shape of clouds remaining rather stable. In these situations, the techniques detecting clouds and their motion trajectories-overall referred to as CMV techniques - provide valuable information for irradiance forecasting. Obviously, the performance of these forecasting methods degrades as the relative importance of local processes of cloud formation and dissipation, such as strong thermally-driven convection, dominate cloud advection.

The following basic steps comprise forecasting based on CMV techniques:

- Images with cloud information are derived from satellite- or ground-based sky-imager measurements.

- Assuming stable cloud structures and optical properties, the CMVs are determined by identifying matching cloud structures in consecutive cloud images.

- To predict future cloud conditions, the CMVs are applied to the latest available cloud image assuming cloud speed persistence.

- Solar irradiance forecasts are calculated from the predicted cloud structures.

\subsubsection{Forecasting Using Ground-Based Sky Imagers}

Solar irradiance forecasts at subhourly scales with high temporal and spatial resolutions can be derived from ground-based sky images. In particular, they have the potential to capture sudden changes in irradiance, often referred to as ramps, on temporal scales of minutes or even less. Cloud fields sensed from sky imagers or from an assemble of sky imagers might be resolved in high details, allowing the partial cloud cover on large PV installations to be modeled and forecasted (see Figure 7-2). The maximum predictable horizon strongly depends 
on cloud conditions-i.e., cloud height and velocity. It is constrained by the cloud speed and the field of view of the sky imager(s). This time horizon is typically less than 15 minutes ahead.
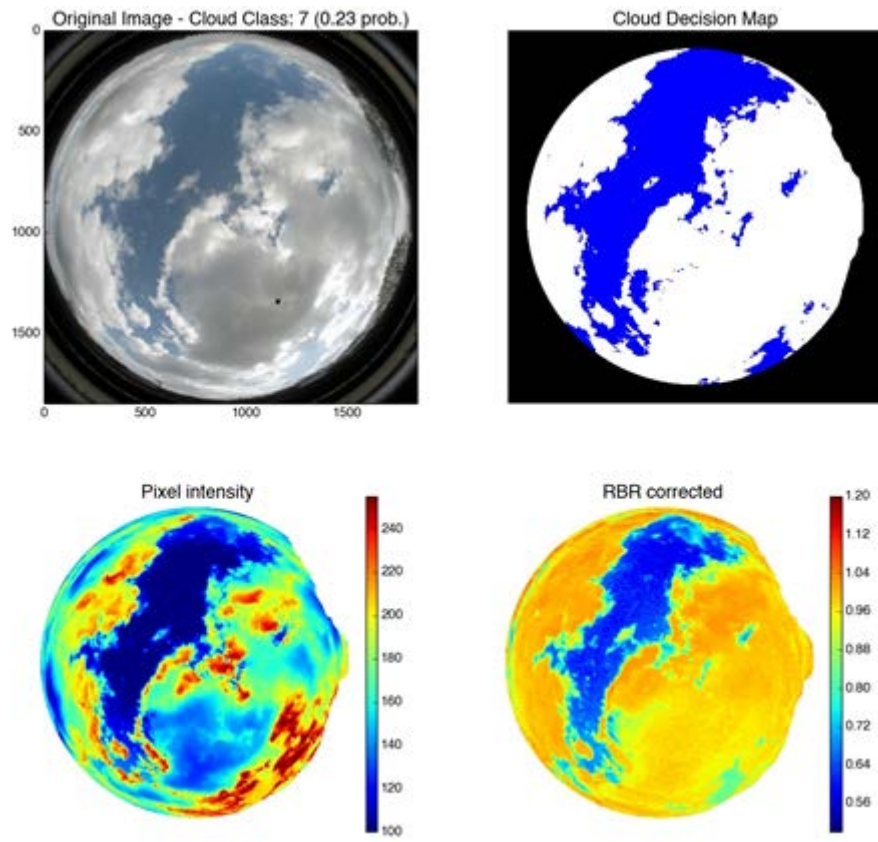

2013-04-19 12:59:00 UTC

Juelich

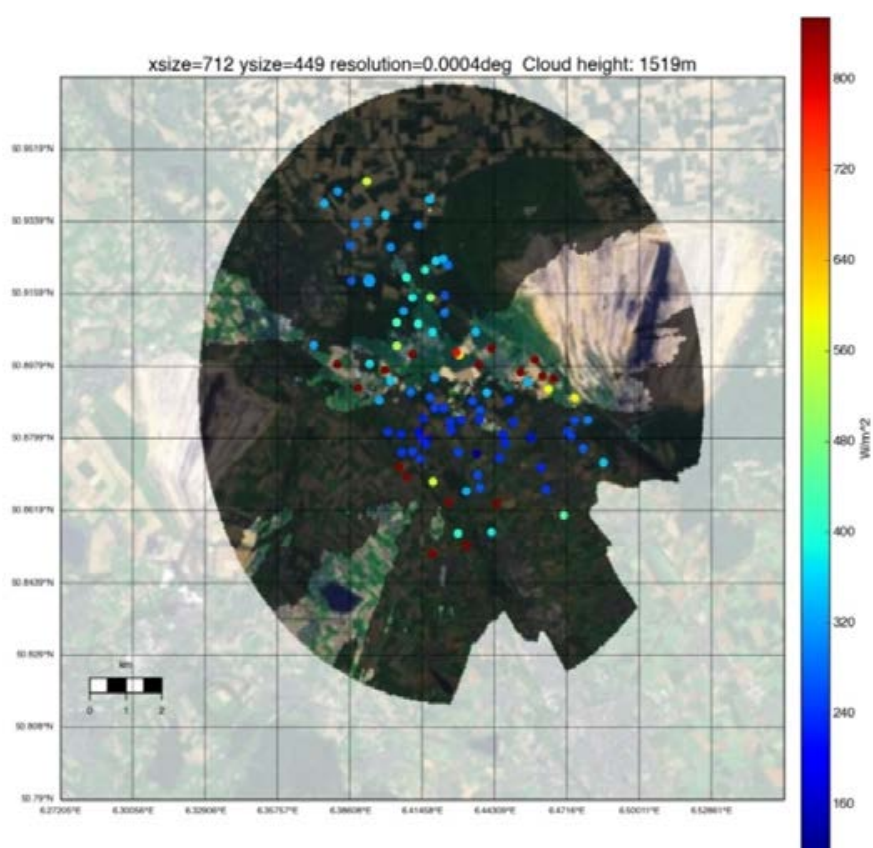

Figure 7-2. Cloud information from sky imagers: upper left, original images; upper right, cloud decision map; middle left, pixel intensity; middle right, red-blue ratio, corrected with a clearsky library; bottom, shadow map with irradiance measurements. Sky image and irradiance measurements were taken in Jülich, Germany, on April 9, 2013, at 12:59 UTC in the framework of the HOPE campaign (Macke and HOPE-Team 2014). Images from the University of Oldenburg

Short-term irradiance forecasting based on ground sky imagers is a rather new research field; a review of the state of the art is given in Urquhart et al. (2013). Currently, there is no defined standard for sky-imaging hardware, camera calibration, or image-processing techniques. 
Systems in use range from commercially available, low-cost, webcam-based, sky cameras to high-quality prototype systems developed at research institutes. These mostly use digital cameras with fish-eye lenses for photography and industry applications - for example, the sky imaging systems specifically designed for solar energy purposes at the University of California at San Diego (Urquhart et al. 2015). Some sky imager forecasts use a single camera and successive evaluation of cloud location, speed, and projection. Integrated threedimensional (3D) forecast systems have been developed more recently (Peng et al. 2015; Oberländer et al. 2015).

Cloud detection (often also referred to as cloud segmentation) from sky-imager observations is performed by evaluating different image properties. The red-to-blue ratio (RBR; Figure 72 , middle right) is often used as a main indicator for clouds because of their different spectral-scattering properties (high RBR) and clear-sky (low RBR) conditions (Shields, Johnson, and Koehler 1993; Long and DeLuisi 1998). Using radiative transfer modeling, Mejia et al. (2016) demonstrated some ambiguity in the RBR method and proposed the radiance RBR method, which leverages intensities and RBR to identify cloudy pixels. Pixel intensities (Figure 7-2, middle left) are also related to cloud cover and might be exploited as an additional feature for cloud detection. Binary cloud decision maps (Figure 7-2, top right) can be derived on the basis of threshold procedures - for example, by evaluating the RBR in relation to a clear-sky library (Chow et al. 2011) to account for nonuniform clear-sky signal over the sky hemisphere that depend on the position of the sun.

Cloud detection is particularly difficult in the circumsolar and solar disk regions because of saturated pixel information that have high RBR values not only for cloudy but also for clearsky conditions. Gauchet et al. (2012) consider the circumsolar area and the solar disk separately with an image segmentation approach, distinguishing also clear skies, bright, and dark clouds. Ghonima et al. (2012) proposed a method to differentiate between thin and thick clouds for various atmospheric conditions using a clear-sky library. Additional information on cloud type in the monitored scene, which also gives an indication on cloud optical thickness and cloud height, can be obtained with cloud classification algorithms or the use of infrared and thermographic sky imagers.

Detecting cloud motion is the next step to derive irradiance forecasts. For instance, Chow et al. (2011) identify cloud motion based on a normalized cross-correlation procedure - i.e., by maximizing the cross-correlation between shifted areas in two consecutive images; whereas Quesada-Ruiz et al. (2014) proposed a discretization method (the sector method) of the cloud image that helps to derive both direction and speed of clouds. Alternatively, cloud movement might be analyzed by applying optical flow techniques to subsequent images, as in Lucas and Kanade (1981) and Wood-Bradely, Zapata, and Pye (2012). The derived cloud motion vectors are then used to cast the observed cloud scenes into the future (Figure 7-3). For pointwise forecasts at the sky-imager location, information about cloud height is not required because the cloud movement can be parameterized in terms of "pixels per second." In contrast, for applications requiring mapping cloud shades, the cloud speed derived using CMVs needs to be expressed in meters per second, which requires knowing the cloud height, which cannot be derived using a sky imager alone.

The multiple options to determine cloud height include ground-based observations, satellite methods, and NWP data. In particular, the most accurate information on cloud-base height is obtained from ceilometers (Arbizu-Barrena et al. 2015), typically employed at airport weather stations; however, the different clouds seen in a sky image can have different cloud heights, and the ceilometer measures only the cloud height directly above it. Thus, the 
applicability of ceilometers for this purpose strongly depends on the particular cloud arrangement. Retrieving the cloud-top height from satellite images (see Section 7.2.3) gives spatially continuous information but shows large uncertainties. Alternatively, different methods to determine cloud height using combined information from more than one sky imager are described in Nguyen and Kleissl (2014); Wang, Kurtz, and Kleissl (2016); and Oberländer et al. (2015); however, they are not exempt from larger uncertainty either. Some of these methods allow deriving different cloud heights for the individual clouds seen in the sky image. Also, the combination of one sky imager's CMV in pixels per second with another device's absolute CMV in meters per second device can be used to determine the cloud height. Examples of such systems are described in Wang, Kurtz, and Kleissl (2016) and Kuhn et al. (2017a).

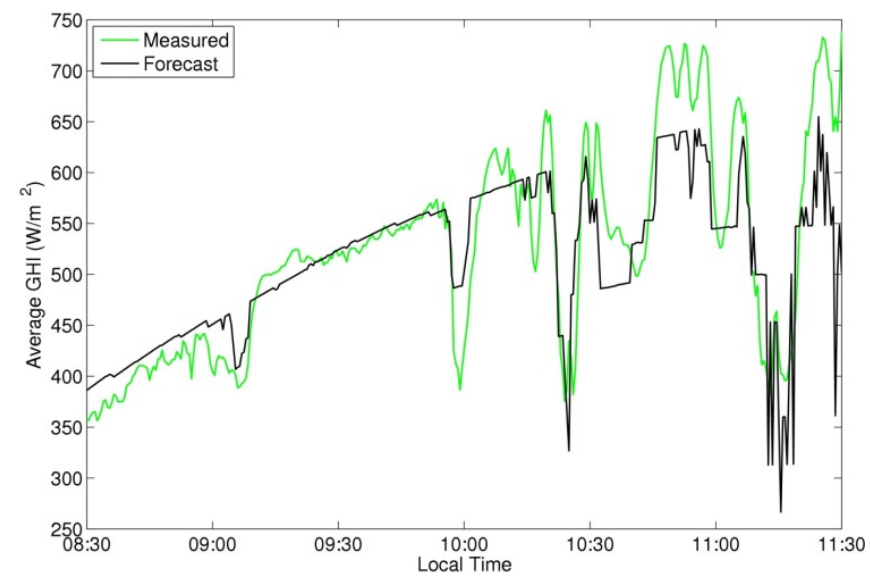

Figure 7-3. Example of 5-minute-ahead GHI forecast using a sky imager. Location: Universtity of California at San Diego, November 14, 2012. Image from University of California at San Diego Center for Energy Research

Cloud shadows maps at the surface (see Figure 7-2, bottom) are produced by projecting the forecasted cloud scenes with their assigned height using information about the position of the sun. The impact of the projection method on solar forecast accuracy can be large. Finally, solar irradiance is estimated from these cloud shadow maps. Without information about cloud optical properties and other atmospheric parameters, this is not a trivial task. Local irradiance or PV power measurements can be used to estimate irradiance or PV power for cloudy and clear skies. Urquhart et al. (2013) analyze frequency distributions of PV power normalized to clear-sky conditions to determine a clear and a cloudy mode and to assign them to shaded and unshaded cells, respectively. Schmidt et al. (2016) use the clear-sky index derived from pyranometer measurements to determine the forecasted all-sky GHI. Similarly, Blanc et al. (2017) uses the beam clear-sky index determined from the last 30 minutes of pyrheliometer measurements to derive the cloud transmittance. Gauchet et al. (2012) proposed using a regression model in combination with a clear-sky model to estimate the surface solar irradiance from segmented sky images with information about clear-sky, bright, and dark clouds; circumsolar area; and solar disk. Deriving GHI and/or DNI from sky images is also discussed in, e.g., Schmidt et al. (2016).

\subsubsection{Satellite-Based Forecasts}

Forecasts up to approximately $6 \mathrm{~h}$ ahead require wide-area observations of cloud fields. For example, assuming a maximum cloud velocity of $160 \mathrm{~km} / \mathrm{h}$, a region of approximately 2,000 $\mathrm{km}$ by $2,000 \mathrm{~km}$ needs to be covered to track arriving clouds 6 hahead. Satellite data with broad coverage are appropriate sources for these horizons. 
Cloud and irradiance information from satellite images can be derived by a variety of methods, as presented in Chapter 4. In principle, all of them can be applied to cloud predictions using CMVs to obtain forecasts of solar irradiance. In addition, there exist multiple methods to derive CMVs, as described in Section 7.2.2 for sky imagers, and as is the case in the realm of operational weather forecasting to describe wind fields at upper levels in the atmosphere.

Satellite-based nowcasting schemes for solar irradiance forecasts have been developed mostly during the past decade based on CMVs or sectoral cloud tracking (Hammer et al. 2003;

Schroedter-Homscheidt and Pulvermüller 2011). The satellite-based forecasting scheme from the University of Oldenburg in Germany (Lorenz et al. 2004; Kühnert, Lorenz, and Heineman 2013 ) is introduced here as an example of such a system. It uses images of the geostationary Meteosat Second Generation (MSG) satellites (see Chapter 4). The semiempirical HELIOSAT method (Hammer et al. 2003) is applied to obtain information about clouds and irradiance. A characteristic feature of the method is the dimensionless cloud index, which gives information about cloud transmissivity.

Cloud motion vectors are derived by identifying corresponding cloud patterns in two consecutive images (Figure 7-4). Rectangular areas, the "target areas," are defined by a size of approximately 90 kilometers by 90 kilometers to be large enough to contain information about temporally stable cloud structures and small enough that cloud motion for this area can be described by a single vector. Mean square pixel differences among target areas in consecutive images $\left(\mathrm{n}_{0}\right.$ and $\left.\mathrm{n}_{-1}\right)$ are calculated for displacements in all directions (Figure 7-4, $\mathrm{a}-\mathrm{c}$ ). The maximum possible displacement ("search area") is determined by the maximum wind speeds at typical cloud heights. The displacement that yields the minimum mean square pixel difference for a given target area is assigned as a motion vector (Figure 7-4, d). The derived motion vectors are applied to the cloud index image $\mathrm{n}_{0}$ to predict future cloud conditions. A smoothing filter is applied to the predicted cloud index image to eliminate randomly varying small-scale structures that are hardly predictable. Finally, the solar irradiance is derived from the predicted cloud index images using the HELIOSAT method (Chapter 4).

The SolarAnywhere short-term forecasting scheme (Perez and Hoff 2013) for the United States based on Geostationary Operational Environmental Satellite (GOES) images follows a similar approach to detect cloud motion and is also based on a semiempirical cloud index method (see Chapter 4). Solargis has developed a CMV short-term forecasting scheme that is run under the same principles already described but incorporates a multiresolution treatment of cloud structures. It is being currently operated over Europe, Africa, and East Asia, and it will be expanded to West Asia and the Americas. Another method presented in SchroedterHomscheidt and Pulvermüller (2011) discriminates tracking optically thin cirrus clouds from tracking optically thick cumulus or stratus clouds with respect to increased accuracy needs in direct irradiance nowcasting for concentrating technologies.

Müller and Remund (2013) proposed a method that combines cloud index values retrieved from MSG satellites with wind fields from a NWP model. The wind fields are predicted with the Weather Research and Forecasting (WRF) model (Skamarock et al. 2005) with hourly resolution and applied to forward propagation of the observed cloud patterns from the satellite imagery. Information about the height of the monitored clouds is necessary to determine the corresponding NWP model level. Müller and Remund (2013) assume fixed cloud heights for this purpose. An advantage of the application of NWP wind fields 
compared to satellite-derived CMVs is the potential to describe changes in the direction and speed of cloud movement during the extrapolation process.

$$
\begin{aligned}
& \text { image } \mathrm{n}_{-1} \text {, } \\
& \mathrm{t}-15 \mathrm{~min}
\end{aligned}
$$
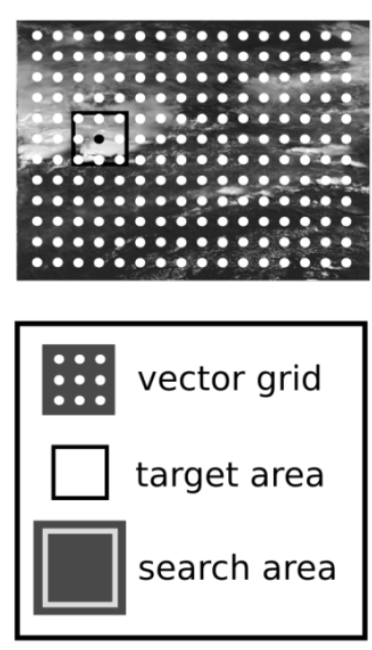

image $n_{0}$, time $\mathrm{t}$

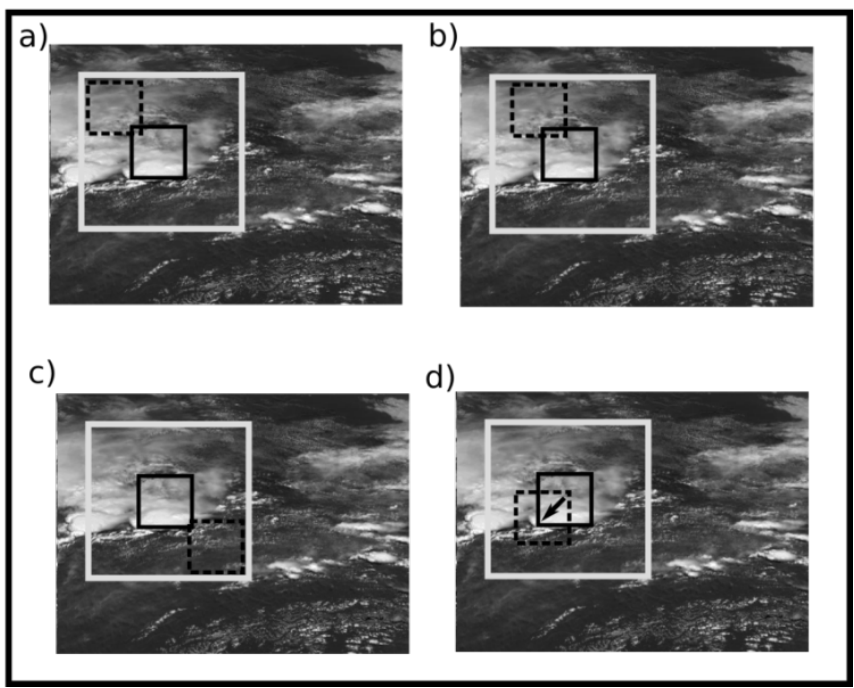

Figure 7-4. Schematic representation of the CMV derivation using satellite images. Images reproduced from Kühnert et al. (2013)

A method for satellite-based short-term forecasting using a physical cloud and irradiance retrieval scheme was introduced by Miller, Heidinger, and Sengupta (2013). The method processes GOES satellite observations with the National Oceanic and Atmospheric Administration (NOAA) Pathfinder Atmospheres Extended (PATMOS-x) retrieval package (Heidinger et al. 2014), which is a stand-alone radiative transfer code, and combines them with wind field data from the Global Forecast System (GFS) model. Cloud properties are retrieved with PATMOS-x in a first step. Next, the cloud fields are advected using GFS winds at the vertical level matching the cloud-top height as retrieved from PATMOS-x. Finally, solar irradiance at the surface is calculated with radiative transfer calculations using predicted cloud properties and additional atmospheric parameters.

\subsubsection{Irradiance Forecasting with Numerical Weather Prediction}

NWP models are routinely operated by weather services to forecast the state of the atmosphere. Starting from initial conditions that are derived from routine observations of the Earth from worldwide networks of ground and airborne sensors, the temporal evolution of the atmosphere is simulated by solving the equations that describe the physical processes occurring in the atmosphere. This physical modeling is the only feasible approach when there is little correlation between the actual observations and the forecasted values, which is typically the case for time horizons longer than approximately $5 \mathrm{~h}$ ahead. A comprehensive overview of NWP modeling is given in Kalnay (2003).

Global NWP models predict the future state of the atmosphere worldwide. To determine the initial state from which a NWP model is run, data assimilation techniques are applied to make efficient use of worldwide meteorological observations (Jones and Fletcher 2013). They include observations from ground-based weather stations, buoys, and spaceborne sensors. The simulation with NWP models involves spatial and temporal discretization, and the resolution of this discretization determines the computational cost of the simulation. In addition, many physical processes occur on spatial scales much smaller than the grid size- 
including, for example, condensation, convection, turbulence, and scattering and absorption of shortwave and longwave radiation. The effect of these unresolved processes on the mean flow at the model's grid size is evaluated with the so-called parameterizations of atmospheric physics. They include interactions of the land and surface with the atmosphere, vertical and temporal development of the planetary boundary layer, cumulus clouds triggering and cloud microphysics, as well as shortwave and longwave radiation. The physical parameterizations are a key component of the prediction with NWP models. They bridge the small-scale and large-scale processes, and they make possible the convergence of the numerical routines that solve the physical equations. Today, global NWP models are run by approximately 15 national and international weather services, and their resolution ranges from approximately $10 \mathrm{~km}-50 \mathrm{~km}$. The temporal resolution of the global model outputs is typically 1 or $3 \mathrm{~h}$, and their forecasts are normally updated every 6 or $12 \mathrm{~h}$.

Mesoscale or regional models cover only a limited area of the Earth. They take the initial and lateral boundary conditions from a previous global NWP model run and bring the spatial and temporal grid of the global NWP model down to a finer resolution. Weather services typically operate mesoscale models with a spatial resolution in the range from $1-10 \mathrm{~km}$ and they provide hourly forecasts, although higher resolutions are feasible. The higher spatial resolution allows for explicit modeling of small-scale atmospheric phenomena.

For irradiance forecasting, the parameterizations of radiation transfer and cloud properties are of special importance. Larson (2013) compares the respective model configurations with respect to GHI for four operational NWP models, including the Integrated Forecast System (IFS) of the European Center for Medium-Range Weather Forecasts (ECMWF) and the GFS run by NOAA of the United States. In particular, Larson (2013) discusses deep and shallow cumulus parameterizations, turbulent transport, stratiform microphysics and prognosed hydrometeors, cloud fraction and overlap assumptions, the description of aerosols, and the shortwave radiative transfer schemes. But he also emphasizes that "because of the strong feedback and interactions of physical processes in the atmosphere," other processes might have a significant impact on irradiance forecasting.

Today, most NWP models offer GHI as direct model output, and some also provide forecasts of direct and diffuse irradiances. Although in principle direct model output can be used for solar energy applications, in practice additional post-processing is customarily applied to improve forecast accuracy.

\subsubsection{Examples of Operational NWP Models}

The following sections describe some examples of NWP models enumerated together with their spatial resolutions and output time intervals, particularly highlighting cloud fraction parameterizations and radiation schemes. Additionally, we provide references with respect to the application and evaluation of irradiance forecasts in the context of solar energy forecasting. A comparison among GHI forecasts based on these models for the United States, Canada, and Europe is given in Perez et al. (2013). It should be emphasized that the sample of operational models and applications given here is not exhaustive; it simply summarizes the research experience and lessons learned from some research completed within the framework of the IEA SHC Task 36 and Task 46.

The IFS of the ECMWF is a global model currently being operated with a horizontal grid spacing of approximately 12 kilometers and 137 vertical levels for high-resolution deterministic forecasts. Operational output is available with a temporal resolution of $3 \mathrm{~h}$ up to 6 days ahead, with a higher resolution of $1 \mathrm{~h}$ accessible in the framework of research projects. 
The model is cycled every $12 \mathrm{~h}$. The radiation code is based on a fast version of the Rapid Radiation Transfer Model for General Circulation Models (RRTMG), adapted for use in NWP models (Mlawer et al. 1997; Iacono et al. 2008). Cloud-radiation interactions are taken Into account in detail by using the values of cloud fraction and liquid, ice, and snow water contents from the cloud scheme using the Monte Carlo Independent Column Approximation (McICA) method (Pincus, Barker, and Morcrette 2003; Morcrette et al. 2008). McICA uses a stochastic approach to infer the cloud extinction of shortwave and longwave solar radiation from only a random selection of calculations. The prognostic scheme for clouds and largescale precipitation is based on Tiedtke (1993). The ECMWF irradiance forecasts are analyzed by Lorenz et al. (2009) with respect to different relevant properties for PV power prediction applications. In addition, Lorenz et al. (2011) propose and evaluate an approach based on the ECMWF forecasts for regional PV power prediction for improved power grid integration.

NOAA's GFS is currently being operated at a spatial resolution of approximately $13 \mathrm{~km}$ and 64 vertical levels; however, the outputs are provided in a regular latitude/longitude grid with a resolution of $0.25^{\circ}$ and 46 levels, with hourly resolution up to $120 \mathrm{~h}$ ahead and $3 \mathrm{~h}$ resolution up to $240 \mathrm{~h}$ ahead. The model is cycled every $6 \mathrm{~h}$. Model physics related to clouds and radiation are summarized in Larson (2013); here, it should be mentioned that cloud fraction is a diagnostic variable in the GFS model in contrast to the IFS model. Mathiesen and Kleissl (2011) give an evaluation of intraday GHI forecasts of the GFS compared to IFS forecasts from the ECMWF and the North American Model.

Environment Canada's Canadian Meteorological Centre operates the Global Environmental Multiscale (GEM) model. It is run in different configurations, including a regional deterministic configuration (Mailhot et al. 2006) generating forecasts up to $48 \mathrm{~h}$ ahead at a 7.5-minute time step and with a spatial resolution of approximately $15 \mathrm{~km}$ at the grid center, in Canada. Pelland, Galanis, and Kallos (2013) investigated solar irradiance and PV power forecasting with post-processing applied to the high-resolution GEM forecasts.

The mesoscale (or regional) WRF model (Skamarock et al. 2005) has been developed in the framework of a long-term collaborative effort of several institutes led by the National Center for Atmospheric Research (NCAR) in the United States. Now it is a community model, meaning that it is publicly and freely available and can receive contributions from anybody. The WRF model is nonhydrostatic, has multiple nesting capabilities, and offers several schemes for each different parameterization of the atmospheric physical processes. This allows the WRF to be adapted to widely different climate conditions and different applications at virtually any region of interest. The shortwave radiation parameterization usually runs the Dudhia (1989) scheme; however, the WRF counts with up to 8 different shortwave parameterization schemes in its latest version (v. 3.9, 2017) including the RRTMG radiative scheme already mentioned for the ECMWF's IFS model but also other advanced and research-class radiative models, such as Goddard (Chou and Suarez 1999), CAM (Collins et al. 2004), and Fu-Liou-Gu (Gu et al. 2011). The user can select any of these schemes. The current WRF's cloud fraction schemes are diagnostic. The impact of the resolved topography on the downward solar radiation can be optionally included in the computations. The direct aerosol impact can be also modeled using built-in climatologies or inputs from the user.

The ability of the WRF model and its precursor, the Mesoscale Model of fifth generation (MM5), to produce solar radiation data have been evaluated in the past (Guichard et al. 2003; Zamora et al. 2003; Zamora et al. 2005; Ruiz-Arias et al. 2008; Wen et al. 2011). More recently, and mostly within the framework of solar energy applications, the WRF model has been profusely evaluated. For instance, within the framework of the IEA SHC Task 36 and 
Task 46, Lara-Fanego et al. (2012) evaluated 3-day-ahead hourly and 10-minute WRF forecasts of GHI and DNI in Spain; Perez and Hoff (2013) conducted a benchmarking study of multiple NWP models, including WRF, throughout European and North American radiometric sites; and Lorenz et al. (2016) recently compared the GHI predictions of multiple models, including WRF, and various model configurations in Europe. Many other studies during the last few years have addressed the model evaluation at different worldwide regions: Isvoranu and Badescu (2015) in Romania; Zempila et al. (2016) in Greece; Aryaputera, Yang, and Walsh (2015) in Singapore; He, Yuan, and Yang (2016) in China; Lima et al. (2016) in Brazil; and Sosa-Tinoco et al. (2016) in Mexico, to name a few.

Other studies have analyzed the causes behind model errors, and some have even proposed improvements. For instance, Mathiesen, Collier, and Kleissl (2013) proposed a direct cloud assimilation technique tailored for the WRF model to improve its representation of clouds along the California coastline for improved solar radiation forecasts. Ruiz-Arias et al. (2013) performed surface clear-sky shortwave radiative closure intercomparisons of various shortwave radiation schemes, including RRTMG, Goodard, and Dudhia in which RRTMG showed the highest performance, whereas some deficiencies were found in the Goddard radiative scheme. A correction for these deficiencies was recently proposed by Zhong, RuizArias, and Kleissl (2016). Ruiz-Arias, Dudhia, and Gueymard (2014) proposed a parameterization of the shortwave aerosol optical properties for surface direct and diffuse irradiances assessment. Later on, Ruiz-Arias et al. (2015) showed the problems of the WRF at simulating convective clouds in the Iberian Peninsula and highlighted the need for a dedicated shallow cumulus scheme to reduce model biases.

An important milestone in the use of the WRF for solar radiation applications has been the recent development of WRF-Solar, a dedicated suite of WRF parameterizations for solar radiation forecasting (Deng et al. 2014; Ruiz-Arias, Dudhia, and Gueymard 2014; Thompson and Eidhammer 2014) within the U.S. Department of Energy project Sun4Cast (Haupt et al. 2016). Some of these improvements, and others, have been summarized in Jimenez et al. (2016b). The Sun4Cast project has contributed also to the development of the Multisensor Advection Diffusion nowCast (MADCast) system (Descombes et al. 2014), which is a particular configuration of the WRF model for fast assimilation of satellite reflectance images to obtain a proxy field to cloud fraction that can be subsequently advected in the WRF and used to compute solar radiation nowcasts. A comparative evaluation of WRF-Solar, MADCast, and satellite-based forecasts is presented in Lee et al. (2017).

The WRF model is operated for solar irradiance forecasting at several public and private entities such as Solargis, Slovakia; Meteotest, Switzerland; GL-Garrad Hassan (Mathiesen, Kleissl, and Collier 2013); and the Atmospheric Sciences Research Center of the University of Albany as part of the operational air quality forecasting program and AWS Truepower in the United States.

The High Resolution Limited Area Model (HIRLAM) ${ }^{1}$ is a hydrostatic regional NWP model operated by several national meteorological services in Europe, such as the Spanish National Weather Service and the Danish Meteorological Institute. The Spanish National Weather Service runs HIRLAM four times per day in three spatial configurations (one covering Europe at a resolution of 16 kilometers and two covering Spain and the Canary Islands, respectively, at a resolution of 5 kilometers) with 40 vertical levels. The Danish Meteorological Institute runs its highest resolution HIRLAM model, "SKA," for an area

\footnotetext{
${ }^{1}$ See http://www.hirlam.org.
} 
covering Northwestern Europe with a grid size of 0.03 degrees (3 kilometers) and 65 vertical levels. HIRLAM uses the clear-sky irradiance scheme of Savijärvi (1990) and the cloud scheme of Wyser, Rontu, and Savijarvi (1999). The nonhydrostatic HARMONIE regional NWP model is being run experimentally by the Spanish Weather Service daily over Spain and the Balearic Islands at a resolution of 2.5 kilometers, with 65 vertical levels.

The regional weather forecasting system SKIRON (Kallos 1997) is operated for solar energy applications at the Spanish National Renewable Energy Center (Gastón et al. 2009).

\subsubsection{Statistical Post-Processing Methods}

Statistical models are used to derive irradiance forecasts based on measurements, usually without involving any physical modeling (time series models with no exogenous input); however, they also play an important role in enhancing the outputs of NWP models and can be applied to CMV forecasts (see Figure 7-5). Different terminology is used for this combination of statistical and physical forecasting methods, depending on the perspective of the researchers. The community of statistical modeling and artificial intelligence refers to these models as statistical models with exogenous input. Meteorologists commonly use the terms statistical post-processing or, more specifically, model output statistics (MOS) in the context of NWP, which is the terminology adopted here.

Post-processing methods are applied to forecast model outputs to:

- Reduce model errors, possibly, by considering unaccounted or partially accounted local and regional effects (e.g., topography and aerosols)

- Derive parameters that are not direct model outputs (i.e., DNI)

- Combine the outputs of different models.

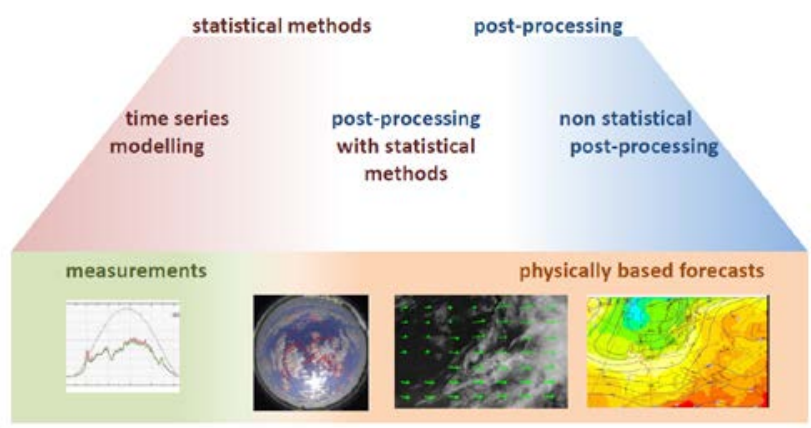

Figure 7-5. Overview of the spectrum of applications of statistical post-processing methods for solar radiation forecasts. Erratum: "Physically based forecasts" should state "CMV-based and NWP-based forecasts."

In the following sections, we give a short overview of different statistical post-processing methods for the highlighted applications.

\subsubsection{Model Output Statistics to Reduce Forecast Errors}

Model output statistics are widely used to refine the output of NWP models, primarily to account for local variations in weather and surface conditions (Glahn and Lowry 1972) using measurements and/or climatology for specific locations as a basis to adapt the forecasts. For example, MOS techniques constitute a powerful tool to adapt the results from NWP or satellite-based models to site-specific conditions (Gueymard et al. 2012). For solar irradiance 
forecasting, satellite-derived values might be used instead of ground measurements. The set of predictors consists of NWP output and might be extended by including any relevant information - for example, prior observations and climatological values.

Originally, the term model output statistics was associated with the use of regression equations; however, a generalization of this concept involves other statistical approaches. Lorenz et al. (2009) applied a bias correction MOS based on solar elevation and clear-sky index to ECMWF irradiance forecasts (see also Section 7.3.7). Kalman filters have also been proposed by Pelland, Galanis, and Kallos (2013) to improve irradiance forecasts of the Canadian GEM model and Diagné et al. (2014) with WRF solar irradiance forecasts. Marquez and Coimbra (2011) investigated the application of artificial neural networks to predicted variables from a weather forecasting database, and Gastón et al. (2009) use a machine-learning algorithm to enhance the SKIRON solar irradiance forecasts. Pierro et al. (2015) proposed a MOS technique to correct WRF GHI forecasts by coupling two different intermediate MOS consisting of correlations with relative humidity and artificial neural networks, respectively.

\subsubsection{Post-Processing to Derive Additional Parameters}

This post-processing method might consist of the use of empirically-based or physicallybased models that compute the desired parameter from the direct outputs of the forecasting model. For instance, Perez et al. (2007) proposed an empirical solar radiation forecast model relating sky-cover predictions from the National Digital Forecast Database to the clear-sky index of global irradiance. The PV power forecasting approaches presented in Lorenz et al. (2011) and Pelland, Galanis, and Kallos (2013) involve empirical models to derive the planeof-array (POA) irradiance as input for PV simulation models. Another common example of empirically-based post-processing is the transposition of the predicted GHI to POA irradiance in PV simulations. This first requires splitting GHI into its direct and diffuse irradiance components. For that purpose, a number of empirical diffuse or direct fraction models are available, originally developed for application to measurements or satellite data. These models are also being used in DNI forecasting systems that are based on a GHI forecast (e.g., Schroedter-Homscheidt, Benedetti, and Killius 2016). Many models have been proposed in the last decades for this purpose. Gueymard and Ruiz-Arias (2015) and Aler et al. (2017) present an unprecedented worldwide evaluation of 140 of these models proposed throughout the last 50 years. Next, the direct and diffuse components are casted to the POA. The transposition of the direct irradiance is straightforward, subject only to geometric considerations. The transposition of the diffuse irradiance requires, again, an empirical model for the directional distribution of radiance over the sky, describing anisotropic effects such as horizon brightening and circumsolar irradiance (Perez et al. 1987; Gueymard 1987; Hay 1979). Validation studies of these transposition models are provided by Behr (1997); David, Lauret, and Boland (2013); Gueymard (2009); Ineichen (2011); and Kambezidis et al. (1994). The validation of combined separation and transposition models has been undertaken by Gueymard (2009); Orehounig, Dervishi, and Mahdavi (2014); Lave et al. (2015); and Yang et al. (2016).

Likewise, physical post-processing approaches can be also used to derive parameters that are not provided as direct model output. Most often, they are required for forecasting DNI and better consideration of aerosols. Breitkreuz et al. (2009) proposed a forecasting approach for direct and diffuse irradiance based on the combination of a chemistry transport model and a NWP model in which forecasts of aerosol optical depth (AOD) are directly collected from the chemistry transport model outputs. These aerosol forecasts together with other remote 
sensing data (ground albedo, ozone) and NWP parameters (water vapor, clouds) are used as input to radiation transfer calculations to derive the irradiance forecasts. A similar approach is used by Lara-Fanego et al. (2012) to derive DNI from the WRF model outputs using aerosol observations from the MODIS sensor onboard the Terra Satellite.

\subsubsection{Combination of Forecast Model Outputs}

Combining the output of different models can increase the forecast accuracy considerably when compared to single-model forecasts. First, simple averaging is beneficial for models with similar accuracy, exploiting the fact that forecast errors of different models are usually not perfectly correlated (Perez et al. 2013).

Combining methods using more advanced techniques might additionally account for strengths and weaknesses of the different models for certain situations - for example, by adapting the contribution of each model depending on the weather situation. In particular, they might be applied to establish a forecast consensus covering horizons from several minutes to several days ahead by integrating measurements, climate monitoring, and NWP forecasts. Various approaches to this aim have been proposed. For instance, Lorenz et al. (2012) used a weighted average with weights optimized for each forecast horizon. Sanfilippo et al. (2015) applied a multi-modeling approach to solar forecasting that uses supervised classification of forecasting evaluation results to select the best predictions from persistence, support vector regression, and diverse stochastic models. Wolff et al. (2016) and Mazorra Aguiar et al. (2017) combine forecasts based on support vector regression machines and artificial neural networks, respectively. Yang et al. (2017) use a hierarchical scheme and minimization of the trace of the forecast error covariance matrix. Within the context of the Sun4Cast project, NCAR's DICast system (Myers et al. 2011; Myers et al. 2012) have been applied to blending multiple solar radiation forecasts. This system-which has already been applied in other forecasting areas, such as transportation, agriculture and wind energyconsists of a two-step process: (1) a statistical bias correction process using a dynamic MOS and (2) optimization of the model blending weights for each lead time (Haupt et al. 2016).

\subsection{Evaluation of Irradiance Forecasts}

The evaluation of solar irradiance forecasts provides users with necessary information about forecast accuracy and helps them to choose different forecasting products or assess the risk when using a particular forecast as a basis for decisions. An extensive overview of forecast verification methods is given in Jolliffe and Stephenson (2003).

The quality of forecasts is evaluated by assessing their similarity to reference data. Most often irradiance measurements are used as reference data-commonly referred to as ground truth data. Nevertheless, reference data are always affected by a certain degree of uncertainty (see Chapter 6). Also, satellite-retrieved irradiance values or the output of a detailed physical model might serve as a reference.

In the following, we give an outline of the most standard evaluation methods, including statistical error measures; comparison to reference models using the skill score parameter; and other important aspects, such as the representation of the observed frequency distribution and the forecast goodness as a function of solar position, hour of the day, cloud variability or even spatiotemporal averaging. We introduce these concepts using examples from an observational data set made of hourly pyranometer measurements from 18 weather stations of the German Weather Service in Germany spanning the period from March 2013 to February 2014 (Lorenz et al. 2016) and forecasts from two NWP models: (1) the high-resolution 
deterministic global IFS model, operated at the ECMWF, with spatial resolution of $0.125,3-$ hourly outputs, and forecast horizon of $24 \mathrm{~h}$ issued every day at 00:00 UTC; and (2) the highresolution regional HIRLAM SKA model, operated at the Danish Meteorological Institute, with spatial resolution of $3 \mathrm{~km}$, hourly outputs, and forecast horizons from 4-9 h ahead, issued every day at 00:00 UTC, 06:00 UTC, 12:00 UTC, and 18:00 UTC.

\subsubsection{Error Measures}

As in the case of measurement data, forecast data should be accompanied by uncertainty information. Such uncertainty data are often derived from the comparison of forecasts to measurements, reference forecasts models, or historical irradiance data. The best practice is to provide at least an analysis of the statistical error measures described in Section 7.3.1.1. Further, the comparison to trivial reference models (Section 7.3.1.2) and a more detailed analysis of the forecast errors as discussed in Section 7.3.2 through Section 7.3.4 is recommended.

\subsubsection{Statistical Error Measures}

Statistical error measures are applied for quantitative forecast evaluation. Here, a number of error measures - the most commonly used-based on first-order statistics are presented.

The error of a single measurement is given as:

$$
\varepsilon_{i}=I_{\text {pred }, i}-I_{\text {meas }, i},
$$

where $I_{\text {pred,i }}$ denotes a predicted irradiance value (GHI or DNI), and $I_{\text {meas }, i}$ is the corresponding measured value.

To evaluate the forecast accuracy of the solar power predictions, it is common practice to use the root mean square error (RMSE):

$$
\operatorname{RMSE}=\frac{1}{\sqrt{N}} \sqrt{\sum_{i=1}^{N} \varepsilon_{i}^{2}}
$$

with $\mathrm{N}$ the number of data pairs. Only daytime values are considered for the evaluation. Relative errors for the irradiance forecast are generally derived by normalization with respect to mean measured irradiance of a given time interval, e.g., one day from sunrise to sunset. In contrast, relative errors of PV power forecasts for utility applications are often normalized to the installed power rather than the mean measured value (e.g., Lorenz et al. 2011).

The RMSE can be split into different parts, related to systematic and stochastic components of forecast errors. The bias:

$$
\text { bias }=\bar{\varepsilon}=\frac{1}{N} \sum_{i=1}^{N} \varepsilon_{i}
$$

is the difference between the mean of predicted and measured values (systematic error). A positive bias means that the predicted values exceed the measurements on average.

The standard deviation of the errors, stderr: 


$$
\text { stderr }=\sigma(\varepsilon)=\frac{1}{\sqrt{N}} \sqrt{\sum_{i=1}^{N}\left(\varepsilon_{i}-\bar{\varepsilon}\right)^{2}}
$$

gives information on the spread of the errors around their mean value. It might be further decomposed into one part related to the error amplitude $\left(\sigma\left(I_{\text {pred }}\right)-\sigma\left(I_{\text {meas }}\right)\right)$ and another part related to the correlation coefficient, $r$, of the time series, defined as:

$$
r=\frac{\sum_{i=1}^{n}\left(I_{\text {pred }, i}-\overline{I_{\text {pred }}}\right) \cdot\left(I_{\text {meas }, i}-\overline{I_{\text {meas }}}\right)}{\sigma\left(I_{\text {pred }}\right) \sigma\left(I_{\text {meas }}\right)}
$$

Overall, the complete decomposition of RMSE yields:

$$
\operatorname{RMSE}^{2}=\text { bias }^{2}+\left(\sigma\left(I_{\text {pred }}\right)-\sigma\left(I_{\text {meas }}\right)\right)^{2}+2 \sigma\left(I_{\text {meas }}\right) \sigma\left(I_{\text {pred }}\right)(1-r)
$$

Some terms of the RMSE can be removed with a simple linear post-processing as $i$ is justified in the following. A linear transformation of a time series $I_{\text {pred }}$ with coefficients $\alpha$ and $\beta$ is given by:

$$
I_{\text {pred }, L R}=\alpha I_{\text {pred }}+\beta
$$

To minimize the RMSE, the mean value and the standard deviation $\sigma\left(I_{\text {pred }}\right)$ of the predicted time series are adjusted in a way that bias vanishes and stderr is minimized (see also Beyer et al. [2009]). The minimum RMSE that can be achieved with a linear transformation, $R M S E_{L R}$, is determined by the standard deviation of the measurements and the correlation $r$ between measurements and predictions:

$$
\mathrm{RMSE}_{\mathrm{LR}}=\sigma\left(I_{\text {meas }}\right) \sqrt{1-r} \text {. }
$$

The standard deviation of the linearly-transformed predictions verifies:

$$
\sigma\left(I_{\text {pred,LR }}\right)=\sigma\left(I_{\text {meas }}\right) r
$$

Therefore, for a perfect correlation between measurements and predictions (i.e., $r=1$ ), $\mathrm{RMSE}_{\mathrm{LR}}$ reaches its minimum possible value (i.e., $\mathrm{RMSE}_{\mathrm{LR}}=0$ ) per Eq. (8-7), and the standard deviation of measurements and the linearly-transformed predictions are the same in virtue of Eq. (8-8). Note that their means are also the same because bias is removed by the linear transformation. In contrast, if the correlation is smaller than one, $\mathrm{RMSE}_{\mathrm{LR}}$ will not be zero anymore and the standard deviation of the linearly-transformed prediction will be smaller than the actual standard deviation of the measurements.

Another common measure to assess forecast accuracy is the mean absolute error:

$$
\mathrm{MAE}=\frac{1}{N} \sum_{i=1}^{N}\left|\varepsilon_{i}\right|
$$

which is recommended in Hoff et al. (2012) as a preferred measure, in particular for reporting relative errors. 
From a user's point of view, ultimately the impact of forecast errors on their application will be decisive for the choice of the most suitable error measure. Mean absolute error (MAE) is appropriate for applications with linear cost functions - i.e., when the costs caused by a wrong forecast are proportional to the forecast error. RMSE is more sensitive to large forecast errors and hence suitable for applications when small errors are more tolerable and larger errors cause disproportionately high costs, which is the case for many applications in the energy market and for grid management issues.

\subsubsection{Skill Score and Persistence Forecast Model}

Skill score (also referred to as forecast skill) is used to quantify the forecast performance compared to a reference model. The parameter normally used for this comparison is RMSE, although others such as MAE or mean square error are often used. The skill score is then defined as the difference between the comparison parameter (e.g., RMSE) for both the reference model and forecast model divided by the difference between the comparison parameter for the reference model and a perfect model (note that a perfect model yields zero RMSE). For the particular case of RMSE, the skill score $\mathrm{SS}_{\mathrm{RMSE}}$ is calculated as:

$$
\mathrm{SS}_{\mathrm{RMSE}}=\frac{\mathrm{RMSE}_{\mathrm{ref}}-\mathrm{RMSE}}{\mathrm{RMSE}_{\mathrm{ref}}},
$$

where $\mathrm{RMSE}_{\text {ref }}$ refers to the reference model and RMSE refers to the investigated forecasting algorithm (Coimbra and Pedro 2013). Its value thus ranges from 1 (perfect forecast) to 0 (reference forecast). A negative value indicates performance worse than the reference. Skill scores might be applied only for comparisons to a simple reference model and also for intercomparisons to different forecasting approaches (improvement scores).

In solar radiation forecasting, the most simple and widely used forecast reference model is persistence. This is a trivial model that assumes the current situation does not change in the future during the forecasted lead time. Various methods derive persistence forecasts. In the following, we present some of the most widely used.

For day-ahead forecasts, the simplest approach is to assume that irradiance I (GHI or DNI) persists. That is:

$$
\mathrm{I}_{\text {per,24h }}(\mathrm{t})=\mathrm{I}_{\text {meas }}(\mathrm{t}-24 \mathrm{~h}) .
$$

A more elaborated option for GHI, which produces more accurate forecasts, is to separate the clear and cloudy contributions to solar radiation and to assume that only the cloudy component (i.e., the random component of GHI) will persist during the forecast lead time. The clear component is deterministic and can be forecasted with reasonable accuracy. In this modeling approach, the persisting magnitude is the clear-sky index, $\mathrm{kt}^{*}{ }_{\text {meas }}$, calculated from the measured GHI. For forecast horizons of several hours $(\Delta t)$ ahead, persistence $G I_{\text {per, } \Delta t}$ for the time, $\mathrm{t}$, is then defined as:

$$
\mathrm{GHI}_{\text {per kt } * \Delta \mathrm{t}}(\mathrm{t})=\mathrm{GHI}_{\text {clear }}(\mathrm{t}) \mathrm{k}_{\text {meas }}^{*}(\mathrm{t}-\Delta \mathrm{t}) .
$$

For day-ahead forecasting, the persistence value of average clear-sky index during the previous day is a more suitable persistence model than the simple approach $\mathrm{GHI}_{\text {per,24h }}$ described above (see Beyer et al. 2009). For DNI, a similar approach can be used based on the beam clear-sky index or the Linke turbidity (Kuhn et al. 2017b). Another reference model much less used is based on climatological mean values. 


\subsubsection{Analysis of Forecast Error with Respect to Solar Elevation}

A special feature of solar irradiance is its very strong deterministic component determined by the daily and seasonal course of the sun. This deterministic signal strongly influences the forecast error signal. Hence, to investigate the solar irradiance forecast errors, sometimes it is advisable to evaluate only the nondeterministic part of solar radiation, primarily caused by errors in the representation of cloud. To this aim, instead of GHI forecast errors, the analyzed variable is often clear-sky index forecast error.

Figure 7-6 shows the RMSE and BIAS of clear-sky index kt* as a function of cosine of solar zenith angle (Figure 8-6, left) and time of the day (Figure 7-6, right) for two different NWP model forecasts (IFS and SKA). The two models show similar behavior: RMSE increases with low solar zenith angles or, equivalently, during morning and evening hours, as is also the case of the magnitude of BIAS. This error pattern is very often caused by a deficient parameterization in the physical models of the atmospheric transport of radiation for low solar altitudes, which is a well-known flaw of the two-stream schemes used in most NWP models as well as other model limitations such as three-dimensional effects and atmospheric refraction issues whose impact is enhanced for low solar altitudes.
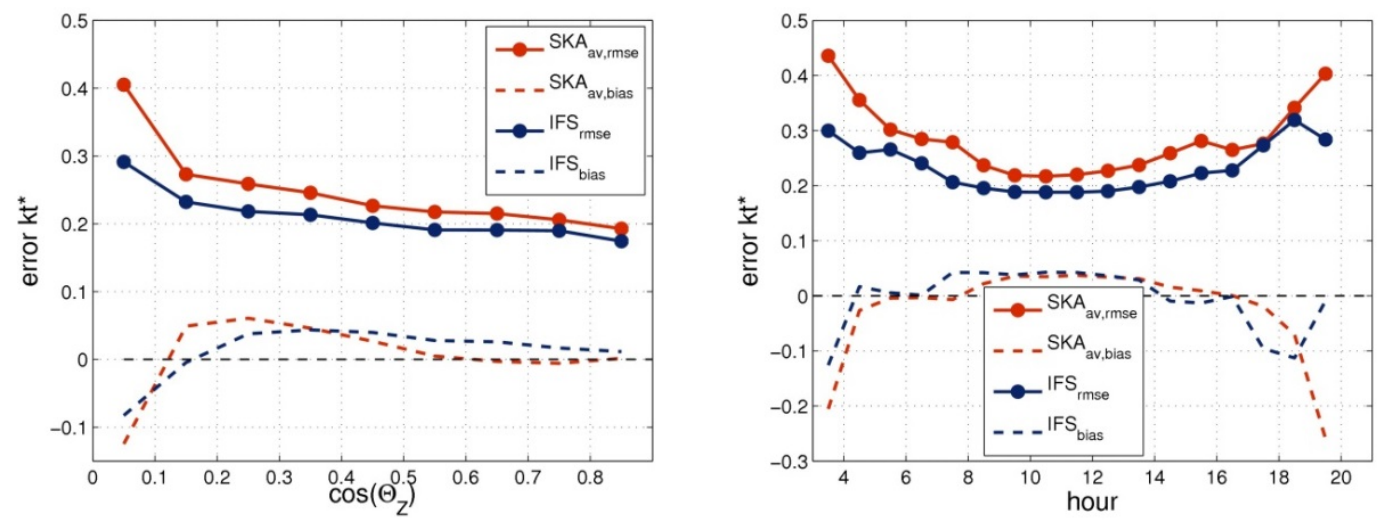

Figure 7-6. Clear-sky forecast error (solid lines for RMSE; dashed lines for mean bias error) as a function of cosine of solar zenith angle (left panel) and hour of the day (right panel) for the forecasts issued by the IFS and SKA NWP models (blue and red lines, respectively). The evaluated period is March 1, 2013-February 28, 2014.

\subsubsection{Analysis of Forecast Error with Respect to Cloud Variability and Spatiotemporal Averaging}

Cloud variability has a strong impact on solar forecasting accuracy. The forecasts generally show good agreement with measurements for clear-sky or even completely overcast days, which basically have a constant clear-sky index. In contrast, considerable deviations from the measurements are typically observed for days with variable clouds. An evaluation of the SKA forecast errors as a function of the variability of clear-sky index of the measurements, here represented by the standard deviation of $\mathrm{kt}^{*}$ meas throughout 5 hours, is shown in Figure 7-7. It also shows this dependence for multiple spatial and temporal averaging configurations of the SKA forecasts. Overall, Figure 7-7 shows:

1. The forecast error grows with enhanced cloud variability.

2. Spatial and temporal forecast averages result in reduced RMSE values, going from negligible reductions for very stable conditions to large reductions for highly variable conditions. 
Regarding the first point, as for the case of solar altitude (Section 7.3.2), the solar radiation forecast error shows a quite clear dependency with respect to cloud variability and, more generally, with respect to the cloudy conditions. The combination of these two error trends has been proposed as an efficient method to reduce the systematic error in the NWP model forecasts using a post-processing MOS (Section 7.2.4.1). In particular, Lorenz et al. (2009) used a polynomial function with cosine of solar zenith angle and clear-sky index as independent variables to parameterize the forecast bias error from historical forecasts compared to observations and subtract the parameterized error from operational forecasts. This approach has been adapted and evaluated also for other NWP models and different climates. Mathiesen and Kleissl (2011) found improved accuracies when applying it to three different NWP models - GFS, North American Model, and IFS - for stations in the continental United States; as did Pelland, Galanis, and Kallos (2013) for the Canadian GEM model; and Müeller and Remund (2013) for the WRF forecasts in Switzerland.

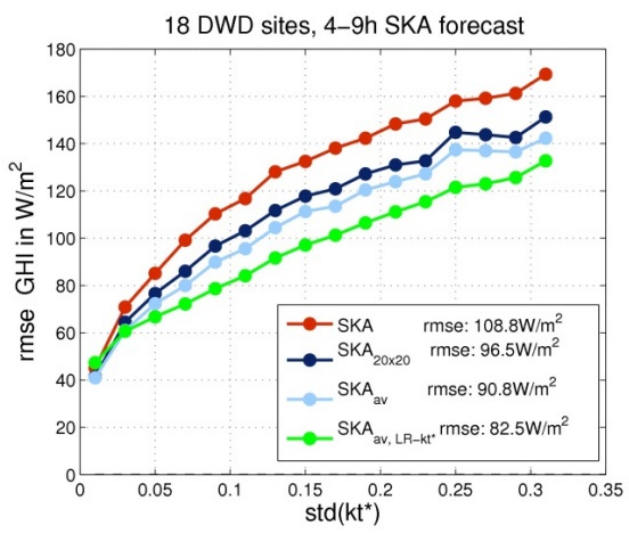

Figure 7-7. RMSE of various versions of the SKA forecasts as a function of the standard deviation of kt*meas([red] SKA: nearest grid point, [dark blue] SKA20x20 averaged throughout 20-by-20 grid points, [light blue] SKAav $5 \mathrm{~h}$ sliding mean of clear-sky index of the average throughout 20-by-20 grid points, and [green] SKAav, LR.kt*: linear regression of kt $^{*}$ applied to SKAAV). The evaluated period is April 3, 2013-February 28, 2014; training set: last 30 days, all sites.

Regarding the second point, the rationale of the RMSE decreases when an averaging scheme is applied is the existence of small correlations among the pixels over which the averaging scheme is applied. This leads to random error cancellations during the averaging process. In contrast, for stable conditions, when the correlation among neighboring pixels is very high, the cancellation of random errors is small.

The optimal region size and time interval for RMSE reduction using averaging depend on the correlation structure among neighboring forecasts, both in time and space. Multiple studies have been conducted in this respect. For instance, a detailed evaluation of irradiance forecasts from the Canadian GEM model resulted in a reduction of forecast errors from $10 \%-15 \%$ when the model outputs were averaged throughout several hundred kilometers (Pelland, Galanis, and Kallos 2013). A similar improvement was achieved for the WRF forecasts provided by Meteotest with averages over an area of $50 \mathrm{~km}$ by $50 \mathrm{~km}$ (Müeller and Remund 2012), whereas Mathiesen and Kleissl (2011) reported $100 \mathrm{~km}$ by $100 \mathrm{~km}$ as a suitable averaging area for irradiance forecasts using the GFS and North American Mesoscale forecast system models. The benefit of horizon-dependent smoothing filters for CMV forecasts is also shown in Lorenz, Hammer, and Heinemann (2004) and Küehnert, Lorenz, and Heineman (2013). 
The reduction of RMSE by spatial and temporal averaging can be extrapolated to the particular case in which the forecasting model performance is evaluated throughout multiple sites along a wide region (also referred to as regional forecast) or for coarser temporal granularities such as monthly and yearly. In these cases, there is a reduction of random errors with respect to point-wise evaluations that make regional forecasting more accurate than point-wise forecasting. Again, the extent of the reduction depends on the particular correlation levels among the aggregated values in each case. An analysis of regional forecast errors for different region sizes and different forecast models is given in Lorenz et al. (2009), Küehnert et al. (2013), and Lorenz and Heinemann (2012).

The temporal and spatial averaging can be also considered for sky imager forecasts. It has been found that in a nowcasting system with four sky imagers and per day with many transient clouds, the DNI RMSE for 10-min-ahead forecasts is reduced from $13.0 \%$ to $6.5 \%$ using averages of $4 \mathrm{~km}^{2}$ and $15 \mathrm{~min}$ with respect to pixel-wise forecasts (Kuhn et al. 2017c).

Despite the goodness of spatiotemporal averaging for reducing the forecast RMSE, it has a collateral effect on the frequency distribution of forecasted data because the averaging step will cut down extreme forecasted values and will distort the original forecast data frequency distribution. As a consequence, averaging forecasts must be used only in applications when a reliable forecast frequency distribution, compared to the observed frequency distribution, is not critical.

\subsubsection{Analysis of the Frequency Distributions of Forecasted Values}

The ability of a model to reproduce the observed frequency distribution of both solar irradiance and clear-sky index is a required property for some applications. In addition, it provides insightful information regarding potential problems in the forecast model.

Figure 7-8 shows the probability density function (PDF) kt* for forecasts of the SKA and IFS NWP models, as in Figure 7-6, and the actual PDF obtained from observations. This plots shows that the SKA model systematically overpredicts clear-sky situations and underpredicts overcast conditions. Consequently, intermediate situations are underrepresented. Oppositely, the IFS model underrepresents very clear and very cloudy conditions and overrepresents intermediate situations.

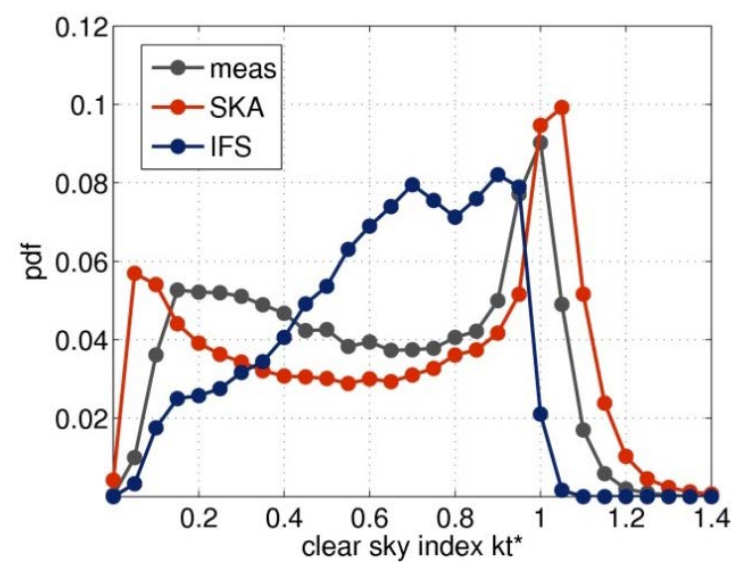

Figure 7-8. Probability density function of the clear-sky index derived from measurements (gray), SKA model forecasts (red), and IFS model forecasts (blue). The evaluated period is March 1, 2013-February 28, 2014; $\cos \left(\Theta_{\mathrm{Z}}\right)>0.1$. 
Although it gives insightful information about the forecast performance, the similarity of the distribution functions of measurements and forecasts does not guarantee an accurate forecast because it does not include information regarding the correct timing of the modeled events.

A quantitative evaluation of the agreement between the observed and forecasted distribution functions can be done using the Kolmogorov-Smirnov integral (Espinar et al. 2008), which is usually applied to distribution functions of GHI or DNI rather than to kt* (Beyer et al. 2009, Perez and Hoff 2013).

\subsection{Recommendations for Solar Irradiance Forecasting}

Forecasting models and methods are continuously evolving, and this makes difficult to elaborate definite guidelines for solar radiation forecasting; however, some broad and general guidelines and recommendations for the selection of solar forecast products aligned with the current state of the art are listed in the following. For the reader particularly interested in DNI nowcasting applications, Ranchin et al. (2017) provide additional recommendations.

- The simultaneous use of independent forecasting methods targeted at the same or different forecast lead times is recommended (e.g., in-situ observations, sky imagers, satellite and NWP, enhanced with statistical methods).

- The forecast products must be validated at various locations featuring different weather and climate conditions, especially those relevant for the site(s) of interest.

- Forecast values should be paired with some sort of uncertainty information, in the form of confidence intervals, quantiles, or similar.

- If available, operational integration of on-site measurements has large potential for forecast improvement, particularly for the shortest forecast time horizons.

- The forecast models should treat cloud extinction of solar radiation as a continuous magnitude. In other words, models treating clouds as binary objects with respect to cloud extinction should be avoided.

- High spatial and temporal resolution forecast products are preferred for wind speed, temperature, and relative humidity, as well as solar radiation for some applications.

With respect to DNI forecast, in addition to the previous recommendations, and given the high sensitivity of DNI to atmospheric aerosols, the following recommendations should also be considered:

- In locations with high temporal variability of aerosols, DNI forecasts should include forecasts of total AOD satellite-based aerosol observations are preferred, especially in areas prone to dust storms.

- In locations with low temporal variability of aerosols or for aerosol nowcasting, monitoring AOD using on-site sun-photometers or pyrheliometers is helpful for modeling DNI. 


\section{References}

Aler, R., I.M. Galván, J.A. Ruiz-Arias, and C.A. Gueymard. 2017. "Improving the Separation of Direct and Diffuse Solar Radiation Components Using Machine Learning by Gradient Boosting." Solar Energy 150:558-569. doi:10.1016/j.solener.2017.05.018.

Almeida, M.P., O. Perpiñán, and L. Narvarte. 2015. "PV Power Forecast Using a Nonparametric PV Model.” Solar Energy 115:354 -368. 10.1016/j.solener.2015.03.006.

Antonanzas, J., N. Osorio, R. Escobar, R. Urraca, F.-J. Martinez-de-Pison, and F. Antonanzas-Torres. 2016. "Review of Photovoltaic Power Forecasting. Solar Energy 136: 78-111. doi; 10.1016/j.solener.2016.06.069.

Arbizu-Barrena, C., D. Pozo-Vázquez, J.A. Ruiz-Arias, and J. Tovar-Pescador. 2015. "Macroscopic Cloud Properties in the WRF NWP Model: An Assessment Using Sky Camera and Ceilometer Data." Journal of Geophyscal Research: Atmospheres 120:1-16. 10.1002/2015JD023502.

Arbizu-Barrena, C., J.A. Ruiz-Arias, F.J. Rodríguez-Benítez, D. Pozo-Vázquez, and J. TovarPescador. 2017. "Short-Term Solar Radiation Forecasting by Advecting and Diffusing MSG Cloud Index. Solar Energy.

Aryaputera, A.W., D. Yang, and W.M. Walsh. 2015. "Day-Ahead Solar Irradiance Forecasting in a Tropical Environment." Journal of Solar Energy Engineering: Transactions of the ASME 137(5):051009.

Bacher, P., H. Madsen, and H.A. Nielsen. 2009. "Online Short-Term Solar Power Forecasting." Solar Energy 83:1,772-1,783.

Behr, H.D. 1997. "Solar Radiation on Tilted South Oriented Surfaces: Validation of Transfer Models.” Solar Energy 61:399-413.

Beyer, H.G., J.P. Martinez, M. Suri, J.L. Torres, E. Lorenz, S.C. Müller, C. Hoyer-Klick, and P. Ineichen. 2009. Management and Exploitation of Solar Resource Knowledge: $\mathrm{Ca}-$ Contract No. 038665-D 1.1.3. Report on Benchmarking of Radiation Products (Technical Report). http://www.mesor.org/docs/MESoR Benchmarking of radiation products.pdf.

Blanc, P., P. Massip, A. Kazantzidis, P. Tzoumanikas, P. Kuhn, S. Wilbert, D. Schüler, C. Prahl. 2017. "Short-Term Forecasting of High Resolution Local DNI Maps with Multiple Fish-Eye Cameras in Stereoscopic Mode." SolarPACES: AIP Proceedings.

Boland, J., Soubdhan, T., 2015. "Spatial-Temporal Forecasting of Solar Radiation." Renewable Energy 75:607-616. doi: 10.1016/j.renene.2014.10.035.

Breitkreuz, H., M. Schroedter-Homscheidt, T. Holzer-Popp, and S. Dech. 2009. "ShortRange Direct and Diffuse Irradiance Forecasts for Solar Energy Applications Based on Aerosol Chemical Transport and Numerical Weather Modeling." Journal of Applied Meteorology and Climatology 48:1,766.

Chou, M.-D., and M.J. Suarez. 1999. A Solar Radiation Parameterization for Atmospheric Studies (Technical Report NASA/TM-1999-104606, vol. 15, Technical Report Series on 
Global Modeling and Data Assimilation). Greenbelt, MD: National Aeronautics and Space Administration Goddard Space Flight Center.

Chow, C.W., et al. 2011. "Intra-Hour Forecasting With a Total Sky Imager at the UC San Diego Solar Energy Testbed." Solar Energy 85(11):2,881-2,893. doi:

10.1016/j.solener.2011.08.025.

Coimbra, C.F.M., and H.T.C. Pedro. 2013. "Stochastic-Learning Methods.” In Solar Energy Forecasting and Resource Assessment, edited by J. Kleiss1, 383-406. Boston: Academic Press. doi: 10.1016/B978-0-12-397177-7.00015-2.

Collins, W.D., et al. 2004. Description of the NCAR Community Atmosphere Model (CAM 3.0) (NCAR Technical Note NCAR/TN-464+ STR, 226). Boulder, CO: National Center for Atmospheric Research.

Dambreville, R., P. Blanc, J. Chanussot, and D. Boldo. 2014. "Very Short-Term Forecasting of the Global Horizontal Irradiance Using a Spatio-Temporal Autoregressive Model." Renewable Energy 72: 291-300. doi: 10.1016/j.renene.2014.07.012.

David, M., P. Lauret, and J. Boland. 2013. "Evaluating Tilted Plane Models for Solar Radiation Using Comprehensive Testing Procedures at a Southern Hemisphere Location." Renewable Energy 51:124-131.

Deng, A., B. Gaudet, J. Dudhia, and K. Alapaty. 2014. "Implementation and Evaluation of a New Shallow Convection Scheme." Paper presented at the WRF: 94th American Meteorological Society Annual Meeting, 26th Conference on Weather Analysis and Forecasting/22nd conference on Numerical Weather Prediction, Atlanta, Georgia, February $2-6$.

Descombes, G., T. Auligné, H.-C. Lin, D. Xu, C. Schwartz, and F. Vandenberghe. 2014. Multisensor Advection Diffusion nowCast (MADCast) for Cloud Analysis and Short-Term Prediction (NCAR Tech. Note NCAR/TN-509+STR). Boulder, CO: National Center for Atmospheric Research. doi:10.5065/D62V2D37.

Diagné, H.M., et al. 2013. "Review of Solar Irradiance Forecasting Methods and a Proposition for Small-Scale Insular Grids." Renewable and Sustainable Energy Reviews 27:65-76. doi: 10.1016/j.rser.2013.06.042.

Diagné, M., M. David, J. Boland, N. Schmutz, and P. Lauret. 2014. "Post-Processing of Solar Irradiance Forecasts from WRF Model at Reunion Island.” Solar Energy 105:99-108.

Dudhia, J. 1989. "Numerical Study of Convection Observed During the Winter Monsoon Experiment Using a Mesoscale Two-Dimensional Model.” Journal of Atmospheric Science 46(20):3077-3107, doi:10.1175/ 1520-046919890463C3077:NSOCOD3E2.0.CO;2.

Dumortier, D. 2009. Management and Exploitation of Solar Resource Knowledge: Contract No. 038665-D 1.2.1 \& D1.2.2 Description of Solar Resource Products: Summary of Benchmarking Results and Examples of Use (Technical Report). http://www.mesor.org/docs/MESoR_Handbook_on_best_practices.pdf. 
Espinar, B., L. Ramirez, A. Drews, H.G. Beyer, L.F. Zarzalejo, J. Polo, and L. Martin. 2008. "Analysis of Different Error Parameters Applied to Solar Radiation Data from Satellite and German Radiometric Stations." Solar Energy 83(1).

Gastón, M., E. Lorenz, S. Lozano, D. Heinemann, M. Blanco, and L. Ramírez. 2009. "Comparison of Global Irradiance Forecasting Approaches." Paper presented at the SolarPACES Conference, Berlin, Germany, September 15-18, 2009.

Gauchet C., P. Blanc, B. Espinar, B. Charbonnier, and D. Demengel. 2012. "Surface Solar Irradiance Estimation with Low-Cost Fish-Eye Camera." Paper presented at COST WIRE workshop on remote sensing measurements for renewable energy, DTU Risoe, Denmark.

German Renewable Energy Sources Act 2017. Accessed on May 2017; https://www.bmwi.de/Redaktion/EN/Dossier/renewable-energy.html

Ghonima M.S., B. Urquhart, C.W. Chow, J.E. Shields, A. Cazorla, and J. Kleissl. 2012. “A Method for Cloud Detection and Opacity Classification Based on Ground-Based Sky Imagery." Atmospheric Measurement Techniques 5:2,881-2,892. doi:10.5194/amt-5-28812012.

Glahn H.R., and D.A. Lowry. 1972. "The Use of Model Output Statistics (MOS) in Objective Weather Forecasting." Journal of Applied Meteorology and Climatology 11:1,203-1,211.

Graditi, G., S. Ferlito, and G. Adinolfi. 2016. "Comparison of Photovoltaic Plant Power Production Prediction Methods Using a Large Measured Dataset," Renewable Energy 90:513-519. doi: 10.1016/j.renene.2016.01.027.

Gu, Y., K.N. Liou, S.C. Ou, and R. Fovell. 2011. "Cirrus Cloud Simulations Using WRF with Improved Radiation Parameterization and Increased Vertical Resolution.” Journal of Geophysical Research 116(D06119). doi:10.1029/2010JD014574.

Gueymard, C.A. 1987. "An Anisotropic Solar Irradiance Model for Tilted Surfaces and Its Comparison with Selected Engineering Algorithms.” Solar Energy 38:367-386.

Gueymard, C.A. 2009. "Direct and Indirect Uncertainties in the Prediction of Tilted Irradiance for Solar Engineering Applications." Solar Energy 83(3): 432-444.

Gueymard, C.A., and J.A. Ruiz-Arias. 2015. "Extensive Worldwide Validation and Climate Sensitivity Analysis of Direct Irradiance Predictions from 1-Min Global Irradiance." Solar Energy 128:1-30. doi: 10.1016/j.solener.2015.10.010.

Gueymard, C.A., W.T. Gustafson, G. Bender, A. Etringer, and P. Storck. 2012. "Evaluation of Procedures to Improve Solar Resource Assessments: Optimum Use of Short-Term Data from a Local Weather Station to Correct Bias in Long-Term Satellite Derived Solar Radiation Time Series." Presented at the ASES-WREF Conference, Denver, Colorado.

Guichard, F., D.B. Parsons, J. Dudhia, and J. Bresch. 2003. "Evaluating Mesoscale Model Predictions of Clouds and Radiation with SGP ARM Data Over a Seasonal Timescale." Monthly Weather Review 131:926-944. 
Hammer, A., D. Heinemann, C. Hoyer, R. Kuhlemann, E. Lorenz, R. Müller, and H.G. Beyer. 2003. "Solar Energy Assessment Using Remote Sensing Technologies." Remote Sensing of Environment 86(3):423-432. doi: 10.1016/S0034-4257(03)00083-X.

Hammer, A., D. Heinemann, E. Lorenz, and B. Lückehe. 1999. "Short-Term Forecasting of Solar Radiation: A Statistical Approach Using Satellite Data." Solar Energy 67(1-3):139_ 150. doi: 10.1016/S0038-092X(00)00038-4.

Haupt, S.E., et al. 2016. The Sun4Cast Solar Power Forecasting System: The Result of the Public-Private-Academic Partnership to Advance Solar Power Forecasting (NCAR Technical Note NCAR/TN-526+STR). Boulder, CO: National Center for Atmospheric Research. doi: 10.5065/D6N58JR2.

Hay, J.E. 1979. Study of Shortwave Radiation on Non-horizontal Surfaces (Technical Report 79-12). Downsview, Ontario: Atmospheric Environment Service.

He, X., C. Yuan, and Z. Yang. 2016. "Performance Evaluation of Chinese Solar Radiation Forecast Based on Three Global Forecast Back Ground Fields." Taiyangneng Xuebao/Acta Energiae Solaris Sinica 37(4):897-904.

Heidinger, A.K., M.J. Foster, A. Walther, and X. Zhao. 2014. "The Pathfinder Atmospheres: Extended AVHRR Climate Dataset." Bulletin of the American Meteorological Society 95:909-922. doi: 10.1175/BAMS-D-12-00246.1.

Hirsch, T., N. Martin, L. Gonzalez, M. Biencinto, S. Wilbert, M. Schroedter-Homscheidt, F. Chenlo, and J.F. Feldhoff. 2014. Technical Report on the Functional Requirements for the Nowcasting Method. Cologne, Germany: DLR Institute of Solar Research, CIEMAT.

Hoff, T.E., R. Perez, J. Kleissl, D. Renne, and J. Stein. 2012. "Reporting of Irradiance Modeling Relative Prediction Errors." Progress in Photovoltaics: Research and Applications 21:1,514-1,519.

Huang, J., M. Korolkiewicz, M. Agrawal, and J. Boland. 2013. "Forecasting Solar Radiation on an Hourly Time Scale Using a Coupled AutoRegressive and Dynamical System (CARDS) Model." Solar Energy 87:136-149. doi: 10.1016/j.solener.2012.10.012.

Iacono, M.J., J.S. Delamere, E.J. Mlawer, M.W. Shephard, S.A. Clough, and W.D. Collins. 2008. "Radiative Forcing by Long-Lived Greenhouse Gases: Calculations with the AER Radiative Transfer Models.” Journal of Geophysical Research 113:D13103. doi:10.1029/ 2008JD009944.

Ineichen, P. 2011. Global Irradiance on Tilted and Oriented Planes: Model Validations (Technical Report). Geneva, Switzerland: University of Geneva. http://archiveouverte.unige.ch/unige:23519.

Inman R.H., H.T.C. Pedro, C.F.M. Coimbra. 2013. "Solar Forecasting Methods for Renewable Energy Integration." Progress in Energy and Combustion Science 39:535-576. doi:10.1016/j.pecs.2013.06.002.

Isvoranu, D., and V. Badescu. 2015. "Comparison Between Measurements and WRF Numerical Simulation of Global Solar Irradiation in Romania." Analele Universitatii de Vest din Timisoara, Seria Fizica LVII:24-33. 
Jimenez, P.A., J.P. Hacker, J. Dudhia, S.E. Haupt, J.A. Ruiz-Arias, C.A. Gueymard, G. Thompson, T. Eidhammer, and A. Deng. 2016a. WRF-Solar: An Augmented NWP Model for Solar Power Prediction. Model Description and Clear-Sky Assessment." Bulletin of the American Meteorological Society 97:1,249-1,264. doi: 10.1175/BAMS-D-14-00279.1.

Jimenez, P.A., S. Alessandrini, S.E. Haupt, A. Deng, B. Kosovic, J.A. Lee, L.D. Monache. 2016b. "The Role of Unresolved Clouds on Short-Range Global Horizontal Irradiance Predictability." Monthly Weather Review 144(9):3,099-3,107. doi: 10.1175/MWR-D-160104.1

Jolliffe I.T., D.B. Stephenson, eds. 2011. Forecast Verification: A Practitioner's Guide in Atmospheric Science: Second Edition. New York: Wiley.

Jones, A.S., and S.J. Fletcher. 2013. "Data Assimilation in Numerical Weather Prediction and Sample Applications." In Solar Energy Forecasting and Resource Assessment, edited by J. Kleissl, 319-355. Boston, MA: Academic Press. doi:10.1016/B978-0-12-397177-7.00013-9.

Kallos, G. 1997. “The Regional Weather Forecasting System SKIRON.” Paper presented at the Symposium on Regional Weather Prediction on Parallel Computer Environments, Athens, Greece, October 15-17.

Kalnay, E. 2003. Atmospheric Modeling, Data Assimilation and Predictability. New York: Cambridge University Press.

Kambezidis, H.D., B.E. Psiloglou, and C.A. Gueymard. 1994. "Measurements and Models for Total Solar Irradiance on Inclined Surface in Athens, Greece.” Solar Energy 53(2):177185.

Kaur, A., L. Nonnenmacher, H.T.C. Pedro, and C.F.M. Coimbra. 2016. "Benefits of Solar Forecasting for Energy Imbalance Markets." Renewable Energy 86:819-830. doi: 10.1016/j.renene.2015.09.011.

Kleissl, J. 2013. Solar Energy Forecasting and Resource Assessment. Boston, MA: Academic Press. doi:10.1016/B978-0-12-397177-7.01001-9.

Kraas, B., M. Schroedter-Homscheidt, and R. Madlener. 2013. "Economic Merits of a Stateof-the-Art Concentrating Solar Power Forecasting System for Participation in the Spanish Electricity Market." Solar Energy 93:244-255.

Kuhn, P., S. Wilbert, C. Prahl, D. Schüler, T. Haase, T. Hirsch, M. Wittmann, R. Pitz- Paal, P. Blanc, L. Ramirez, L. Zarzalejo, A. Meyer, and L. Vuilleumier. 2017a. "Shadow Camera System for the Generation of Solar Irradiance Maps." Solar Energy 157: 157-170.

Kuhn, P., S. Wilbert, D. Schüler, C. Prahl, T. Haase, L. Ramirez, L. Zarzalejo, A. Meyer, L. Vuilleumier, P. Blanc, J. Dubrana, A. Kazantzidis, M. Schroedter-Homscheidt, T. Hirsch, and R. Pitz-Paal. 2017b. "Validation of Spatially Resolved All Sky Imager Derived DNI Nowcasts." Proceedings of SolarPACES2016.

Kuhn, P., S. Wilbert, C. Prahl, A. Kazantzidis, L. Ramirez, L. Zarzalejo, L. Vuilleumier, P. Blanc, and R. Pitz-Paal. 2017c. "Shadow Camera System for the Validation of Nowcasted Plant-Size Irradiance Maps." Paper presented at the European Meteorological Society Annual Meeting, Dublin, Ireland, September 4-8.Kühnert, J., E. Lorenz, and D. Heineman. 2013. 
"Satellite-Based Irradiance and Power Forecasting for the German Energy Market." In Solar Energy Forecasting and Resource Assessment, edited J. Kleissl, 267-297. Boston, MA: Academic Press. doi:10.1016/B978-0-12-397177-7.00011-5.

Kurtz, B., and J. Kleissl. 2017 "Measuring Diffuse, Direct, and Global Irradiance Using a Sky Imager.” Solar Energy 141:311-322.

Lara-Fanego, V., J.A. Ruiz-Arias, D. Pozo-Vázquez, F.J. Santos-Alamillos, J. TovarPescador. 2012. "Evaluation of the WRF Model Solar Irradiance Forecasts in Andalusia (Southern Spain).” Solar Energy 86(8):2,200-2,217. doi: 10.1016/j.solener.2011.02.014.

Larson V.E. 2013. "Forecasting Solar Irradiance with Numerical Weather Prediction Models." In Solar Energy Forecasting and Resource Assessment, edited by J. Kleissl, 299318. Boston, MA: Academic Press.

Lave, M., W. Hayes, A. Pohl, and C.W. Hansen. 2015. "Evaluation of Global Horizontal Irradiance to Plane of Array Irradiance Models at Locations across the United States." IEEE Journal of Photovoltaics 99:1-10.

Law, E.W., A.A. Prasad, M. Kay, and R.A. Taylor. 2014. "Direct Normal Irradiance Forecasting and Its Application to Concentrated Solar Thermal Output Forecasting: A Review." Solar Energy 108:287-307. doi: 10.1016/j.solener.2014.07.008.

Law, E.W., M. Kay, and R.A. Taylor. 2016. "Evaluating the Benefits of Using Short-Term Direct Normal Irradiance Forecasts to Operate a Concentrated Solar Thermal Plant." Solar Energy 140:93-108. doi: 10.1016/j.solener.2016.10.037.

Lee, J.A., S.E. Haupt, P.A. Jimenez, M.A. Rogers, S.D. Miller, and T.C. McCandless. 2017. "Solar Irradiance Nowcasting Case Studies Near Sacramento." Journal of Applied Meteorology and Climatology 56(1):85-108. doi: 10.1175/JAMC-D-16-0183.1.

Lima, F.J.L., F.R. Martins, E.B. Pereira, E. Lorenz, and D. Heinemann. 2016. "Forecast for Surface Solar Irradiance at the Brazilian Northeastern Region Using NWP Model and Artificial Neural Networks." Renewable Energy 87:807-818.

Long, C.N., and J.J. DeLuisi. 1998. "Development of an Automated Hemispheric Sky Imager for Cloud Fraction Retrievals." Paper presented at the 10th Symposium on Meteorological Observations and Instrumentation, Phoenix, Arizona, January 11-16, pp. 171-174.

Lonij, V.P.A., A.E. Brooks, A.D. Cronin, M. Leuthold, and K. Koch. 2013. "Intra-Hour Forecasts of Solar Power Production Using Measurements from a Network of Irradiance Sensors." Solar Energy 97:58-66. doi: 10.1016/j.solener.2013.08.002.

Lorenz, E., A. Hammer, and D. Heinemann. 2004. "Short-Term Forecasting of Solar Radiation Based on Satellite Data." Paper presented at the EUROSUN2004, ISES Europe Solar Congress, Freiburg, Germany.

Lorenz, E., and D. Heinemann. 2012. "Prediction of Solar Irradiance and Photovoltaic Power." Comprehensive Renewable Energy 1:239-292. doi:10.1016/B978-0-08-0878720.00114-1. 
Lorenz, E., J. Kühnert, D. Heinemann, K.P. Nielsen, J. Remund, and S.C. Müller. 2016. "Comparison of Global Horizontal Irradiance Forecasts Based on Numerical Weather Prediction Models with Different Spatio-Temporal Resolutions." Progress in Photovoltaics: Research and Applications 24(12):1,626-1,640.

Lorenz, E., J. Remund, S.C. Müller, W. Traunmüller, G. Steinmaurer, D. Pozo, J.A. RuizArias, V.L. Fanego, L. Ramirez, M.G. Romeo, C. Kurz, L.M. Pomares, C.G. Guerrero. 2009. "Benchmarking of Different Approaches to Forecast Solar Irradiance." Paper presented at the 24th European Photovoltaic Solar Energy Conference, Hamburg, Germany, September 2125 .

Lorenz, E., T. Scheidsteger, J. Hurka, D. Heinemann, and C. Kurz. 2011. "Regional PV Power Prediction for Improved Grid Integration." Progress in Photovoltaics: Research and Applications 19:757-771. doi:10.1002/pip.1033

Lucas, B., and T. Kanade. 1981. "An Iterative Image Registration Technique with an Application to Stereovision." Paper presented at the Imaging Understanding Workshop, Pittsburgh, Pennsylvania.

Macke, A., and HOPE-Team. 2014. "The $\mathrm{HD}(\mathrm{CP})^{2}$ Observational Prototype Experiment HOPE: An Overview." ACP Special Issue: $H D(C P)^{2}$ Observational Prototype Experiment.

Mailhot, J., S. Belair, L. Lefaivre, B. Bilodeau, M. Desgagne, C. Girard, A. Glazer, A.M. Leduc, A. Methot, A. Patoine, A. Plante, A. Rahill, T. Robinson, D. Talbot, A. Tremblay, P.A. Vaillancourt, and A. Zadra. 2006. "The 15-km Version of the Canadian Regional Forecast System.” Atmosphere-Ocean 44:133-149.

Marcos et al. 2013. "Attenuation of Power Fluctuations in Large PV Power Plants: The Use of Prediction to Optimize the Storage System." Paper presented at the 28th European Photovoltaic Solar Energy Conference (PVSEC), September $30^{\text {th }}$-October $4^{\text {th }}$, Paris, France.

Marquez, R., and C.F.M. Coimbra. 2011. "Forecasting of Global and Direct Solar Irradiance Using Stochastic Learning Methods, Ground Experiments and the NWS Database." Solar Energy 85(5):746-756. doi: 10.1016/j.solener.2011.01.007.

Marquez, R., and C.F.M. Coimbra. 2013. "Intra-Hour DNI Forecasting Based on Cloud Tracking Image Analysis.” Solar Energy 91:327-336. doi:10.1016/j.solener.2012.09.018.

Marquez, R., H.T.C. Pedro, and C.F.M. Coimbra. 2013. "Hybrid Solar Forecasting Method Uses Satellite Imaging and Ground Telemetry as Inputs to ANNs." Solar Energy 92:176-188. doi: 10.1016/j.solener.2013.02.023.

Martinez-Anido, C.B., B. Botor, A.R. Florita, C. Draxl, S. Lu, H.F. Hamann, and B.-M. Hodge. 2016. "The Value of Day-Ahead Solar Power Forecasting Improvement." Solar Energy 129:192-203.

Mathiesen, P., and J. Kleissl. 2011. "Evaluation of Numerical Weather Prediction for IntraDay Solar Forecasting in the Continental United States." Solar Energy 85(5):967-977. doi:10.1016/j.solener.2011.02.013. 
Mathiesen, P., C. Collier, and J. Kleissl. 2013. "A High-Resolution, Cloud-Assimilating Numerical Weather Prediction Model for Solar Irradiance Forecasting." Solar Energy 92:47_ 61.

Mathiesen, P., J. Kleissl, and C. Collier. 2013. "Case Studies of Solar Forecasting with the Weather Research and Forecasting Model at GL-Garrad Hassan." In Solar Energy Forecasting and Resource Assessment, edited by J. Kleiss1, 357-382. Boston, MA: Academic Press. doi:10.1016/B978-0-12-397177-7.00014-0.

Mazorra Aguiar, L., B. Pereira, P. Lauret, F. Díaz, and M. David. 2016. "Combining Solar Irradiance Measurements, Satellite-Derived Data and a Numerical Weather Prediction Model to Improve Intra-Day Solar Forecasting." Renewable Energy 97:599-610. doi:

10.1016/j.renene.2016.06.018.

Mejia, F.A., B. Kurtz, K. Murray, L.M. Hinkelman, M. Sengupta, Y. Xie, and J. Kleissl. 2016. "Coupling Sky Images with Radiative Transfer Models: A New Method to Estimate Cloud Optical Depth." Atmospheric Measurement Techniques 9:4,151-4,165. doi: 10.5194/amt-9-4151-2016.

Miller, S.D., A.K. Heidinger, and M. Sengupta. 2013. "Physically Based Satellite Methods." In Solar Energy Forecasting and Resource Assessment, edited by J. Kleissl, 49-79. Boston, MA: Academic Press. doi:10.1016/B978-0-12-397177-7.00003-6.

Mlawer, E.J., S.J. Taubman, P.D. Brown, M.J. Iacono, and S.A. Clough. 1997. "Radiative Transfer for Inhomogeneous Atmospheres: RRTM, a Validated Correlated-k Model for the Longwave." Journal of Geophysical Research 102 :16,663-16,682. doi:10.1029/97JD00237.

Morcrette, J., H.W. Barker, J.N. Cole, M.J. Iacono, and R. Pincus. 2008. "Impact of a New Radiation Package, McRad, in the ECMWF Integrated Forecasting System." Mon. Wea. Rev., 136:4,773-4,798. doi: 10.1175/2008MWR2363.1.

Müller, S.C., and J. Remund. 2014. "Satellite Based Shortest Term Solar Energy Forecast System for Entire Europe for the Next Hours." $29^{\text {th }}$ European Photovoltaic Solar Energy Conference and Exhibition, September 22 - September 26, Amsterdam, The Netherlands. doi: 10.4229/EUPVSEC20142014-5BV.1.6.

Myers, W., G. Wiener, S. Linden, and S.E. Haupt. 2011. “A Consensus Forecasting Approach for Improved Turbine Hub Height Wind Speed Predictions.” Paper presented at WindPower 2011, Anaheim, California, May 24, 2011.

Myers, W., S. Linden, G. Wiener, and S.E. Haupt. 2012. "A Consensus Wind Forecasting System." Paper presented at the World Renewable Energy Forum, Denver, Colorado, May 17.

Nguyen, D., and J. Kleiss1. 2014. "Stereographic Methods for Cloud Base Height Determination Using Two Sky Imagers.” Solar Energy 107:495-509.

Oberländer, D., C. Prahl, S. Wilbert, S. Müller, B. Stanicki, and N. Hanrieder. 2015. "Cloud Shadow Maps from Whole Sky Imagers and Voxel Carving." Presented at the ICEM, June 23 -26 , Boulder, Colorado. 
Ohtake, H., K. Shimose, J. Gari da Silva Fonseca Jr., T. Takashima, T. Oozeki, and Y. Yamada. 2013. "Accuracy of the Solar Irradiance Forecasts of the Japan Meteorological Agency Mesoscale Model for the Kanto Region, Japan." Solar Energy 98(Part B):138-152. doi:10.1016/j.solener.2012.10.007.

Orehounig, K., S. Dervishi, and A. Mahdavi. 2014. "Computational Derivation of Irradiance on Building Surfaces: An Empirically-Based Model Comparison." Renewable Energy 71:185-192.

Pelland, S., G. Galanis, and G. Kallos. 2013. "Solar and Photovoltaic Forecasting through Post-Processing of the Global Environmental Multiscale Numerical Weather Prediction Model." Progress in Photovoltaics: Research and Applications 21:284-296. doi:10.1002/pip.1180.

Peng, Z., D. Yu, D. Huang, J. Heiser, S. Yoo, and P. Kalb. 2015. "3D Cloud Detection and Tracking System for Solar Forecast Using Multiple Sky Imagers.” Solar Energy 118:496519. doi: 10.1016/j.solener.2015.05.037.

Perez, R., A. Kankiewicz, J. Schlemmer, K. Hemker, and S. Kivalov. 2014. “A New Operational Solar Resource Forecast Model Service for PV Fleet Simulation.” Paper presented at the IEEE 40th Photovoltaic Specialist Conference (PVSC), June 8 - 13, Denver, Colorado. doi: 10.1109/PVSC.2014.6925204

Perez, R., and T. Hoff. 2013. "SolarAnywhere Forecasting." In Solar Energy Forecasting and Resource Assessment, edited by J. Kleissl, 233-265. Boston, MA: Academic Press. doi:10.1016/B978-0-12-397177-7.00010-3.

Perez, R., E. Lorenz, S. Pelland, M. Beauharnois, G. Van Knowe, K. Hemker Jr., D. Heinemann, J. Remund, S.C. Müller, W. Traunmüller, G. Steinmauer, D. Pozo, J.A. RuizArias, V. Lara-Fanego, L. Ramirez-Santigosa, M. Gaston-Romero, and L.M. Pomares. 2013. "Comparison of Numerical Weather Prediction Solar Irradiance Forecasts in the U.S., Canada and Europe.” Solar Energy 94:305-326. doi:/10.1016/j.solener.2013.05.005.

Perez, R., K. Moore, S. Wilcox, D. Renné, and A. Zelenka. 2007. "Forecasting Solar Radiation: Preliminary Evaluation of an Approach Based Upon the National Forecast Database." Solar Energy 81(6):809-812. doi:10.1016/j.solener.2006.09.009.

Perez, R., R. Seals, P. Ineichen, R. Stewart, and D. Menicucci. 1987. “A New Simplified Version of the Perez Diffuse Irradiance Model for Tilted Surfaces." Solar Energy 39(3):221231. doi:10.1016/S0038-092X(87)80031-2.

Perez, R., S. Kivalov, J. Schlemmer, K. Hemker Jr., D. Renné, T.E. Hoff. 2010. "Validation of Short and Medium Term Operational Solar Radiation Forecasts in the U.S." Solar Energy 84(12):2,161-2,172. doi: 10.1016/j.solener.2010.08.014

Pierro, M., F. Bucci, C. Cornaro, F. Maggioni, A. Perotto, M. Pravettoni, and F. Spada. 2015. "Model Output Statistics Cascade to Improve Day Ahead Solar Irradiance Forecast." Solar Energy 117:99-113.

Pincus, R., H.W. Barker, and J.-J. Morcrette. 2003. “A Fast, Flexible, Approximate Technique for Computing Radiative Transfer in Inhomogeneous Cloud Fields.” Journal of Geophysical Research 108(D13):4376. doi:10.1029/2002JD003322. 
Quesada-Ruiz, S., Y. Chu, J. Tovar-Pescador, H.T.C. Pedro, and C.F.M. Coimbra. 2014. "Cloud-Tracking Methodology for Intra-Hour DNI Forecasting." Solar Energy 102:267-275. doi:10.1016/j.solener.2014.01.030.

Ranchin, T., S. Wilbert, M. Schroedter-Homscheidt, and Y.-M. Saint-Drénan. 2017. Best Practice Guideline for DNI Nowcasting (DNICast Project Report).

Reikard, G. 2009. "Predicting Solar Radiation at High Resolutions: A Comparison of Time Series Forecasts." Solar Energy 83(3):342-349. doi:10.1016/j.solener.2008.08.007.

Rikos, E., S. Tselepis, C. Hoyer-Klick, and M. Schroedter-Homscheidt. 2008. "Stability and Power Quality Issues in Microgrids under Weather Disturbances: An Implementation to the Kythnos Island Power System." IEEE Journal of Special Topics in Earth Observations and Remote Sensing 1:170-179. doi:10.1109/JSTARS.2008.2010557.

Ruiz-Arias, J.A., C. Arbizu-Barrena, F.J. Santos-Alamillos, J. Tovar-Pescador, and D. PozoVazquez. 2015. "Assessing the Surface Solar Radiation Budget in the WRF Model: A SpatioTemporal Analysis of the Bias and its Causes." Monthly Weather Review 144:703-711. doi: 10.1175/MWR-D-15-0262.1.

Ruiz-Arias, J.A., D. Pozo-Vázquez, N. Sánchez-Sánchez, J.P. Montávez, A. Hayas-Barrú, and J. Tovar-Pescador. 2008. "Evaluation of Two MM5-PBL Parameterizations for Solar Radiation and Temperature Estimation in the South-Eastern Area of the Iberian Peninsula." Il Nuovo Cimento C 31(5-6):825-842. doi: 10.1393/ncc/i2009-10343-6.

Ruiz-Arias, J.A., J. Dudhia, and C.A. Gueymard. 2014. "A Simple Parameterization of the Short-Wave Aerosol Optical Properties for Surface Direct and Diffuse Irradiances Assessment in a Numerical Weather Model." Geoscientific Model Development 7:593-629. doi: 10.5194/gmd-7-1159-2014.

Ruiz-Arias, J.A., J. Dudhia, F.J. Santos-Alamillos, and D. Pozo-Vázquez. 2013. "Surface Clear-Sky Shortwave Radiative Closure Intercomparisons in the Weather Research and Forecasting Model.” Journal of Geophysical Research: Atmospheres 118. doi:10.1002/jgrd.50778.

Sanfilippo, A., L. Martin-Pomares, N. Mohandes, D. Perez-Astudillo, and D. Bachour. 2016. "An Adaptive Multi-Modeling Approach to Solar Nowcasting." Solar Energy 125(February):77-85. ISSN 0038-092X, https://doi.org/10.1016/j.solener.2015.11.041.

Savijärvi, H. 1990. "Fast Radiation Parameterization Schemes for Mesoscale and ShortRange Forecast Models.” Journal of Applied Meteorology 29:437-447, doi:10.1175/15200450(1990)029<0437:FRPSFM>2.0.CO;2.

Schmidt, T., J. Kalisch, E. Lorenz, and D. Heinemann. 2016. "Evaluating the SpatioTemporal Performance of Sky-Imager-Based Solar Irradiance Analysis and Forecasts." Atmospheric Chemistry and Physics 16:3,399-3,412. doi:10.5194/acp-16-3399-2016.

Schroedter-Homscheidt, M., A. Oumbe, A. Benedetti, and J.-J. Morcrette. 2013. "Aerosols for Concentrating Solar Electricity Production Forecasts: Requirement Quantification and ECMWF/MACC Aerosol Forecast Assessment." Bulletin of the American Meteorological Society 94(6):903-914. doi: 10.1175/BAMS-D-11-00259.1. 
Schroedter-Homscheidt, M., and A. Benedetti, and N. Killius. 2016. "Verification of ECMWF and ECMWF/MACC's Global and Direct Irradiance Forecasts with Respect to Solar Electricity Production Forecasts." Meteorology Z. doi:/10.1127/metz/2016/0676.

Schroedter-Homscheidt, M., and B. Pulvermüller. 2011. "Verification of Direct Normal Irradiance Forecasts for the Concentrating Solar Thermal Power Plant Andasol-3 Location." Paper presented at SolarPACES, September 20 - 23, Granada, Spain.

Schroedter-Homscheidt, M., and S. Wilbert. 2017. "Methods to Provide Meteorological Forecasts for Optimum CSP Systems Operations." In The Performance of Concentrated Solar Power (CSP) Systems: Analysis, Measurement and Assessment-1st Edition, edited by Peter Heller. Woodhead Publishing, 2017, Pages 253-281, ISBN 9780081004470 , https://doi.org/10.1016/B978-0-08-100447-0.00008-0. (http://www.sciencedirect.com/science/article/pii/B9780081004470000080).

Shields, J.E., R.W. Johnson, and T.L. Koehler. 1993. "Automated Whole Sky Imaging Systems for Cloud Field Assessment." Paper presented at the Fourth Symposium on Global Change Studies, 17-22 January, American Meteorological Society, Boston.

Simoglou, C.K.,et al. 2014. "An Advanced Model for the Efficient and Reliable Short-Term Operation of Insular Electricity Networks with High Renewable Energy Sources Penetration." Renewable and Sustainable Energy Reviews 38:415-427. doi: 10.1016/j.rser.2014.06.015.

Skamarock, W.C., J.B. Klemp, J. Dudhia, D.O. Gill, D.M. Barker, W. Wang, J.G. Powers. 2005. A Description of the Advanced Research WRF Version 2 (NCAR Technical Note NCAR/TN-468+STR). Boulder, CO: National Center for Atmospheric Research.

Sosa-Tinoco, I., J. Peralta-Jaramillo, C. Otero-Casal, A. López-Agüera, G. Miguez-Macho, and I. Rodríguez-Cabo. 2016. "Validation of a Global Horizontal Irradiation Assessment from a Numerical Weather Prediction Model in the South of Sonora-Mexico." Renewable Energy 90:105-113.

Thompson, G., and T. Eidhammer. 2014. "A Study of Aerosol Impact on Clouds and Precipitation Development in a Large Winter Cyclone.” Journal of Atmospheric Sciences 71:3,636-3,658.

Tiedtke, M. 1993. "Representation of Clouds in Large-Scale Models." Monthly Weather Review 121:3,040-3,061.

Urquhart, B., B. Kurtz, E. Dahlin, M. Ghonima, J.E. Shields, and J. Kleissl. 2015. "Development of a Sky Imaging System for Short-Term Solar Power Forecasting." Atmospheric Measurement Techniques 8(2):875-890. doi:10.5194/amt-8-875-2015.

Urquhart, B., M. Ghonima, D. Nguyen, B. Kurtz, C.W. Chow, and J. Kleissl. 2013. "SkyImaging Systems for Short-Term Forecasting." In Solar Energy Forecasting and Resource Assessment, edited by J. Kleissl, 195-232. Boston, MA: Academic Press. doi:10.1016/B9780-12-397177-7.00009-7.

Voyant, C., C. Darras, M. Muselli, C. Paoli, M.-L. Nivet, and P. Poggi. 2014. "Bayesian Rules and Stochastic Models for High Accuracy Prediction of Solar Radiation." Applied Energy 114:218-226. doi: 10.1016/j.apenergy.2013.09.051. 
Wang, G., B. Kurtz, and J. Kleiss1. 2016. "Cloud Base Height from Sky Imager and Cloud Speed Sensor.” Solar Energy 131:208-221.

Wen, X., S. Lu, L. Shang, Y. Ao, Y. Bao, and W. Li. 2011. "Numerical Simulations of Radiation Budget Using WRF Model over the Jinta Oasis-Gobi." Taiyangneng Xuebao/Acta Energiae Solaris Sinica 32(3):346-353.

Wittmann, M., H. Breitkreuz, M. Schroedter-Homscheidt, and M. Eck. 2008. "Case Studies on the Use of Solar Irradiance Forecast for Optimized Operation Strategies of Solar Thermal Power Plants." IEEE Journal of Selected Topics in Applied Earth Observations and Remote Sensing 1(1):18-27. doi: 10.1109/JSTARS.2008.2001152.

Wolff, B., J. Kühnert, E. Lorenz, O. Kramer, and D. Heinemann. 2016. “Comparing Support Vector Regression for PV Power Forecasting to a Physical Modeling Approach Using Measurement, Numerical Weather Prediction and Cloud Motion Data." Solar Energy 135:197-208. doi: 10.1016/j.solener.2016.05.051.

Wood-Bradley, P., J. Zapata, and J. Pye. 2012. "Cloud Tracking with Optical Flow for ShortTerm Solar Forecasting." Paper presented at the $50^{\text {th }}$ Conference of the Australian Solar Energy Society, Melbourne, December..

Wyser, K., L. Rontu, and H. Savijarvi. 1999. "Introducing the Effective Radius into a Fast Radiation Scheme of a Mesoscale Model. " Contributions to Atmospheric Physics 72(3):205218.

Yang, D. 2016. "Solar Radiation on Inclined Surfaces: Corrections and Benchmarks." Solar Energy 136:288-302. doi:10.1016/j.solener.2016.06.062.

Yang, D., H. Quan, V.R. Disfani, and L. Liu. 2017. "Reconciling Solar Forecasts: Geographical Hierarchy.” Solar Energy 146:276-286.

Zamora, R., S. Solomon, E. Dutton, J. Bao, M. Trainer, R. Portmann, A. White, D. Nelson, and R. McNider. 2003. "Comparing MM5 Radiative Fluxes with Observations Gathered During the 1995 and 1999 Nashville Southern Oxidants Studies.” Journal of Geophysical Research 108:4050. doi: 10.1029/2002JD002122.

Zamora, R.J., E.G. Dutton, M. Trainer, S.A. McKeen, J.M. Wilczak, and Y.-T. Hou. 2005. "The Accuracy of Solar Irradiance Calculations Used in Mesoscale Numerical Weather Prediction." Monthly Weather Review 133:783-792.

Zempila, M.-M., T.M. Giannaros, A. Bais, D. Melas, and A. Kazantzidis. 2016. "Evaluation of WRF Shortwave Radiation Parameterizations in Predicting Global Horizontal Irradiance in Greece.” Renewable Energy 86:831-840.

Zhong, X., J.A. Ruiz-Arias, and J. Kleissl. 2016. "Dissecting Surface Clear-Sky Irradiance Bias in Numerical Weather Prediction: Application and Corrections to the New Goddard Shortwave Scheme.” Solar Energy 132:103-113. doi: 10.1016/j.solener.2016.03.009. 


\title{
8 Applying Solar Resource Data to Solar Energy Projects
}

\author{
Lourdes Ramírez, ${ }^{1}$ Chris Gueymard, ${ }^{2}$ Dave Renné, ${ }^{3}$ Kristian Pagh Nielsen, ${ }^{4}$ \\ Luis Zarzalejo, ${ }^{5}$ Stefan Wilbert, ${ }^{6}$ and Aron Dobos ${ }^{7}$ \\ 1. Centro de Investigaciones Energéticas, Medioambientales y Tecnológicas \\ (CIEMAT) \\ 2. Solar Consulting Services \\ 3. Dave Renné Renewables, LLC \\ 4. Danish Meteorological Institute \\ 5. Centro Investigaciones Energéticas, Medioambientales y Tecnológicas \\ 6. German Aerospace Center (DLR) \\ 7. Envision Energy, Ltd.
}

\subsection{Introduction to Solar Resources for Solar Energy Projects}

As discussed in previous chapters, solar resource evaluation covers a wide range of topics and applications. Most of these applications are related to projects involving solar radiation energy conversion. In what follows, these are referred to as "solar energy projects" and include electricity production applications (photovoltaics, solar thermal electricity), solar heating applications (central solar heating for district heating, local domestic heating and cooling), water and air applications (disinfection, desalination, decontamination), and energy conservation (for building applications).

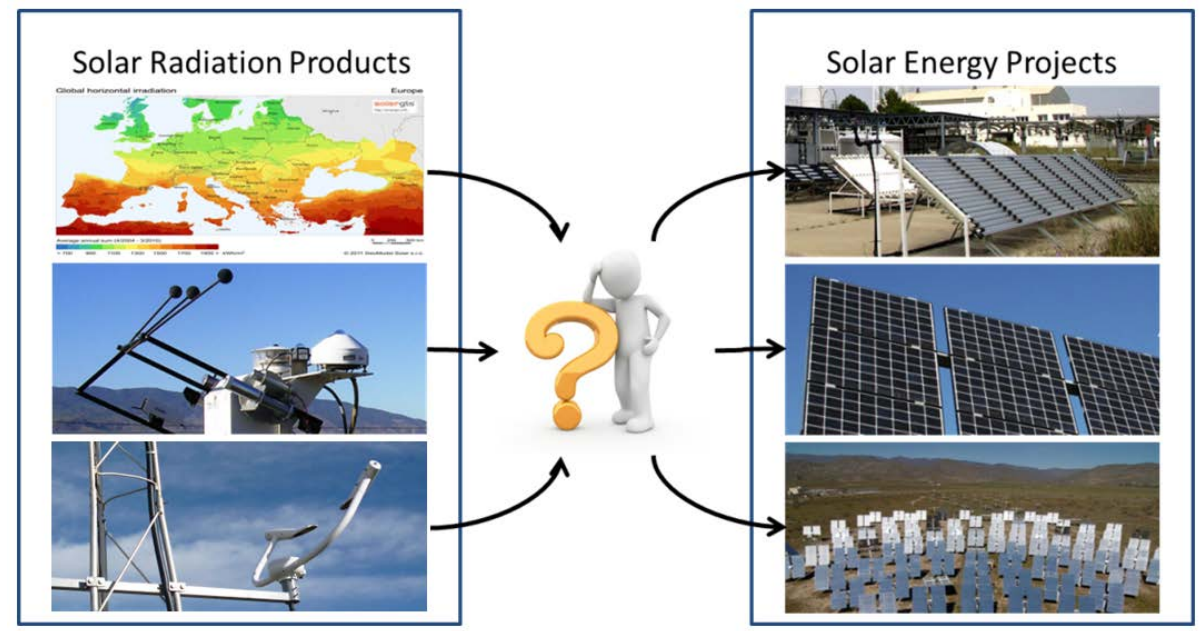

Figure 8-1. Different solar radiation products or evaluation methodologies (left) described in previous chapters can be applied to solar energy projects (right). Image by Ramirez

The overall goal in applying solar resource data to solar energy projects is to help the project developer or investor identify the best estimates or methodology to get the optimal solar resource and weather information to address each one of the project stages. Hence, this chapter summarizes all available information as well as guidance on the type of solar resource relevant to each of these stages. In addition, some information about how to generate data sets for energy simulations is also provided. Details on project stages are developed more thoroughly in Sections 2-5, corresponding to what is shown in Figure 8-2. In Section 6, special needs of solar resource data for different types of solar projects are included. Finally, Section 7 provides a summary for further reference. 


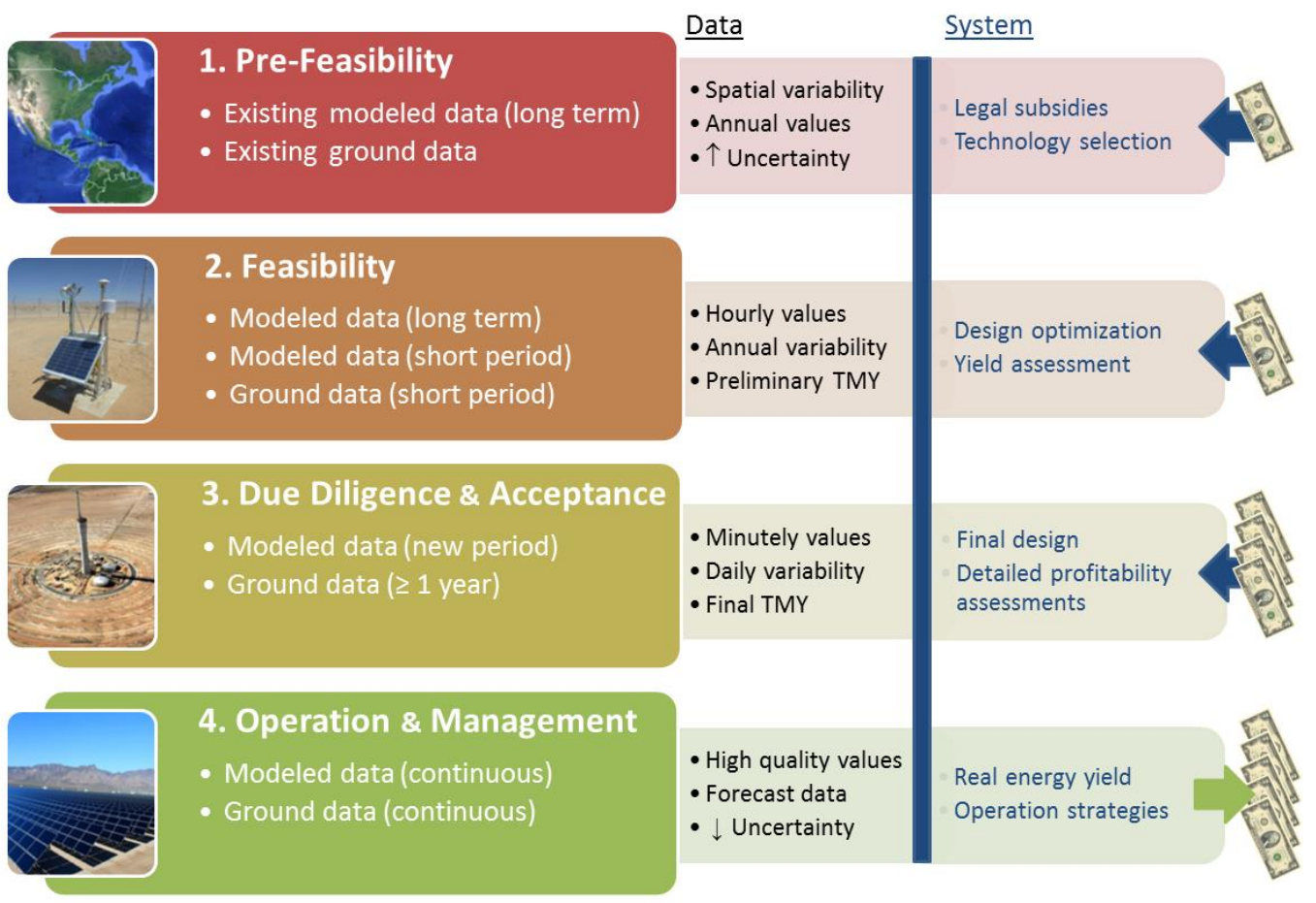

Figure 8-2. Solar radiation needs at four stages of a solar power project. Pictures from www:

(1) Google Earth view; (2) CSP Services measurement station of low maintenance requirements; (3) Gemasolar CSP 20MW 15 hours of storage capacity under construction; (4) Copper Mountain PV 552 MW. Image by Ramirez

The exact needs for solar resource data for a project depend on the project characteristics and how it is financed. Typically, a large solar power project will require several years of highquality modeled data and at least one year of on-site measured data at the final stages of the project execution. The on-site data have to be collected using the measurement procedures described in Chapter 3, in formats directly relevant to the type of technology being considered. The modeled data can be obtained from one of the sources reviewed in Chapter 4 and 5

For the first stages of the project execution, project developers can rely on a number of information sources. In most countries, solar radiation data sources include limited on-site information of varying quality, such as:

- Nearby measurements that may or may not be precisely applicable to the site because of spatial and temporal variability

- Satellite-derived irradiance estimates

- Estimations from reanalysis of numerical weather prediction (NWP) models.

Assuming that no high-quality on-site data are available during the site screening and prefeasibility stages, energy estimates must be derived from these three sources or improved data sets from commercial vendors. During feasibility assessments, including engineering analysis and due diligence, some period (one year or more) of high-quality measurements are assumed to be available at the site. However, these relative short-term measurements have to be used by the solar resource provider and combined with long-term modeled data to ultimately derive a long-term record that removes the bias in the original modeled time series, 
while still capturing seasonal trends and the inter-annual variability of solar resources for the site. This merging process is usually referred to as "site adaptation" and is described in Section 8.3.3. During the project's operation stage, on-site high-quality ground-based measurements are normally necessary to evaluate the performance of the system in real time. These measurements can be supplemented to some extent, or in some cases replaced, by ongoing estimations such as satellite-derived data sets for the region or for the specific site.

The project developer should consult Figure 8-3 throughout the stages of project development. In a solar power project, some questions have to be addressed at each stage, as presented in the following sections. Sections 8.1.1-8.1.4 provide some specific information that could help in the interpretation of Figure 8-3 and also present topics that are addressed in Sections 8.2-8.5.

\subsubsection{Site Selection for Solar Energy Projects}

At the first stages of a project, some questions related to its exact location may still be open.

- What proposed site location(s) need(s) to be evaluated?

- Has a single site been chosen?

- Is the developer making a choice from among two or more sites or still "prospecting" from a wider area?

If choosing among multiple sites, the developer would benefit from using maps and other graphical techniques to evaluate the estimated resource, as well as its variability and uncertainty.

- What are the temporal and spatial characteristics of the data sources available to the developer, and how do these characteristics influence the evaluation of system performance?

Regarding temporal characteristics, measured solar data apply to a specific location and are usually recorded at short time intervals $(1-10 \mathrm{~min})$; then they are averaged to the desired time interval (often hourly in the early project phase). Modeled data such as satellite-derived data usually represent snapshots in time because of the scanning characteristics of spaceborne radiometers and are typically considered to represent 5-60-minute averages.

For most of the modeled gridded databases derived from geosynchronous satellite imagery, the individual pixel (or cell) size is in the range of $1-10 \mathrm{~km}$ but depends on the specific model configuration, the specific instrument, and on the pixel's geographic location.

\subsubsection{Predicted Plant Output Throughout its Project Life}

The important questions that need to be addressed here are as follows:

- What will be the energy produced during each year (or even each month) of the project's life?

- How is a time series of solar radiation data generated for the energy simulation of a specific project?

- How can data sets provide projections throughout the next 20-30 years so that the cash flow (revenue minus expenses) can be evaluated throughout the life of the project? 
Use of a typical meteorological year (TMY) (Marion and Urban 1995) is a popular method for solar system simulation. Much commercial or public-domain energy simulation software relies on TMYs to describe the hourly characteristics of the local solar resource. By design, however, a TMY only represents average or "median" conditions and, thus, does not provide information about the real variability or possible extremes through the system lifetime. Note, moreover, that different locations may have more or less inter-annual variability. For instance, locations subject to monsoon effects will have high inter-annual variability during summer. Typically, on-site data are not available for more than one year at the final stage of the project preparation. Time series of satellite-derived modeled data covering the last 15-25 years are available from various (mostly commercial) providers. Longer-term (up to 55 years) modeled irradiance data sets are available from only a number of sites such as from the NRSDB in the United States. ${ }^{1}$ Long measured time series covering many decades also exist but for only a few stations in the world.

\subsubsection{Solar Radiation Needs During Plant Construction or Due Diligence Processes}

Solar radiation information is necessary to answer new questions at this stage:

- How reliable is the plant output prediction?

- What is the expected uncertainty of the solar radiation estimations?

- What is the margin of error in the annual (or monthly) cash-flow estimate?

Once the plant is under construction, different situations can occur. In a case where the developer is not the final owner or is not in charge of the operations and maintenance (O\&M), a due diligence is included in the evaluation process. For this, solar energy consultancy services should be requested by each financial investor. The predictions of plant output that were made previously can now be updated if new solar radiation data (measurements or modeled estimates) are available. Even if the developer is the final owner, the uncertainty in the production results can be updated. All solar resource predictions that have been obtained from possibly different consultancy services must be compared and the corresponding uncertainties must be deemed in reasonable agreement.

\subsubsection{System Operating Strategies and Actual Performance}

The following situations are frequent and must be addressed:

- What kind of irradiance data should be used to conduct studies on grid integration, load matching, or system intermittency?

In this case, daily, hourly, or sub-hourly data are typically needed for a specific time period, which cannot be provided by TMY data.

- How importantly can the temporal variability in the solar resource affect the plant's revenue?

Most large solar energy projects are built to produce electricity for the public utility grid. Most utilities buy electricity from producers at different rates during the day, depending on their load pattern, which can also vary on a seasonal basis. It is thus in the interest of the plant owner and operator to maximize electricity dispatch when its value is largest. Hence, time-ofday pricing vs. production potential becomes an essential variable to be optimized. This is

\footnotetext{
${ }^{1}$ See http://rredc.nrel.gov/solar/old_data/nsrdb/.
} 
possible using storage systems, and it is also related to maintenance scheduling. This constitutes an extension of the general situation described above and requires much more information than just the estimate of the annual average production based on a TMY. For example, if a solar power plant includes significant storage capacity, a complex daily analysis including measured on-site irradiance and forecasts is needed to determine when the system will fill storage, to which level, and when it will rather directly provide power to the grid during daylight hours. Storage greatly mitigates the effect of system intermittency, but accurate real-time on-site measurements are additionally needed in order to take the best decisions related to O\&M. In such an example, accurate solar forecasts up to one day ahead, continuously improved by the real-time data, actually constitute the main tool to guide the decision process. Forecasts for more than one day ahead are required, for example, for electricity market participation and maintenance scheduling. In extreme cases, it is also possible that the local electric utility has to disrupt the normal production schedule of solar power plants equipped with storage and ask their operators to increase or decrease production to avoid grid instability. This is, again, a situation that requires good real-time irradiance measurements and solar forecasts. 


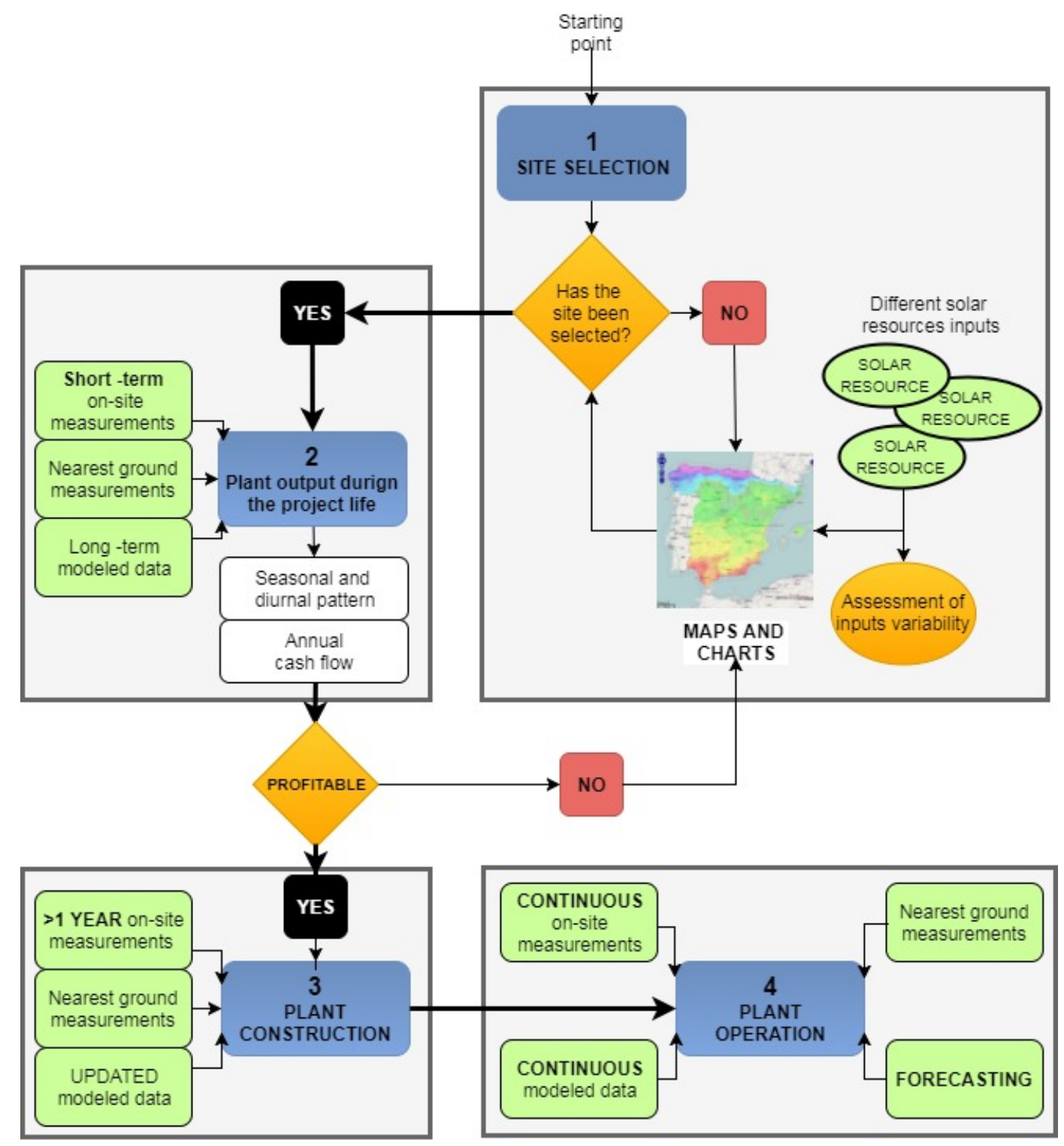

Figure 8-3. Flowchart of the solar radiation data needs (in green) for a solar power project. Image by Ramirez

\subsection{Data Applications for Site Screening and Prefeasibility Assessment}

\subsubsection{The Site Screening Process}

In the early stages of project development, a prefeasibility assessment of possible sites is undertaken. A desired outcome at this stage is the estimated annual energy production that could be expected from the solar energy system in various proposed locations. Historical solar resource data sets are generally used at this stage, often in the form of maps, or from publicly available or commercial gridded data like those discussed in Chapter 4 and 5 . These data sets use a fairly consistent methodology to reliably identify the regions of highest solar potential. The maps should be used to make a preliminary assessment of the solar resource, assuming a relatively large potential for error (up to $\approx 15 \%$, depending on the data provider and region). Thus, if a desirable level of solar resource for a solar plant project is a daily mean of $7.0 \mathrm{kWh} / \mathrm{m}^{2}$, sites with mapped resource values down to approximately $6.0 \mathrm{kWh} / \mathrm{m}^{2}$ 
should be considered. Typically, concentrating solar projects require a much larger solar resource than non-concentrating technologies. Hence, PV projects may still be economically viable even if the solar resource is relatively low, inasmuch as the power production has a sufficient value on the market.

A "first order" prefeasibility assessment includes the analysis of potential for various technologies. In the case of solar thermal electricity (STE) technologies, such studies were conducted for the southwestern United States by the National Renewable Energy Laboratory's (NREL's) Concentrating Solar Power Program (Mehos and Perez 2005), ${ }^{2}$ for instance. Using geographic information system (GIS) screening techniques, resource maps were developed that highlighted regions potentially suitable for project development after considering various land-use constraints - such as protected land areas, sloping terrain, and distance from transmission (Figure 8-4). The results of these studies show that, even with these constraints, vast areas in the southwestern United States are potentially suitable for STE development. Maps such as these have been valuable to project developers to highlight specific regions in which various levels of site prospecting and prefeasibility analysis can take place.

Other studies are now done by various groups to evaluate the solar potential of PV installations on building roofs at the scale of a specific city. Such studies require GIS data at very high resolution (better than $1 \mathrm{~m}$ ), which are usually provided by light detection and ranging (LiDAR) techniques and sophisticated shading analyses (Huang et al. 2015; Jakubiec and Reinhart 2013; Le et al. 2016; Martínez-Rubio et al. 2016; Mohajeri et al. 2016; Santos et al. 2014; Tooke et al. 2012).

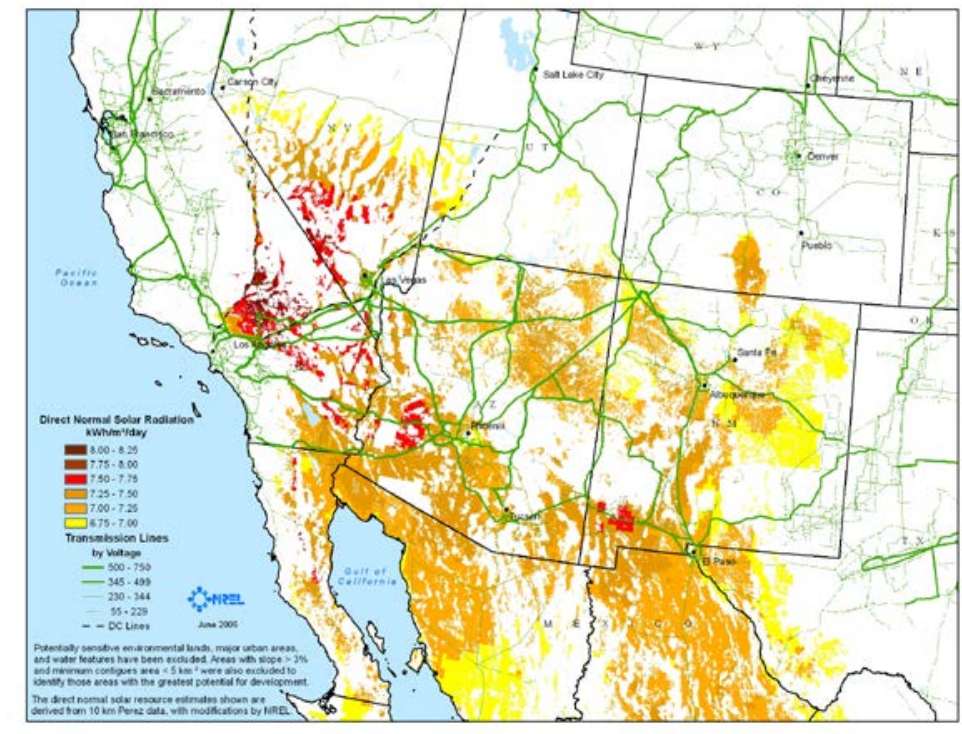

Figure 8-4. CSP prospects of the southwest United States. GIS analysis for available site selection using direct normal irradiance (DNI) resource, land use, and $3 \%$ terrain slope. Image by NREL (Mehos and Perez 2005)

Navarro et al. (2016) made a comparison of STE potential assessment methodologies and showed the need for providing intercomparable results, while pointing out the importance of some constraints like the terrain's slope. A methodology called land constraints, radiation,

\footnotetext{
${ }^{2}$ See www.nrel.gov/csp/data-tools.html.
} 
and slope considerations (LRS) was proposed, harmonizing the treatment of these three main inputs. Figure $8-5$ shows how the slope consideration (1\%, $2 \%$, or $3 \%$ of maximum slope) affects the site selection for what became a real STE power plant in Spain. Only when accepting a maximum slope of $3 \%$, the whole power plant was eventually placed in a suitable area.
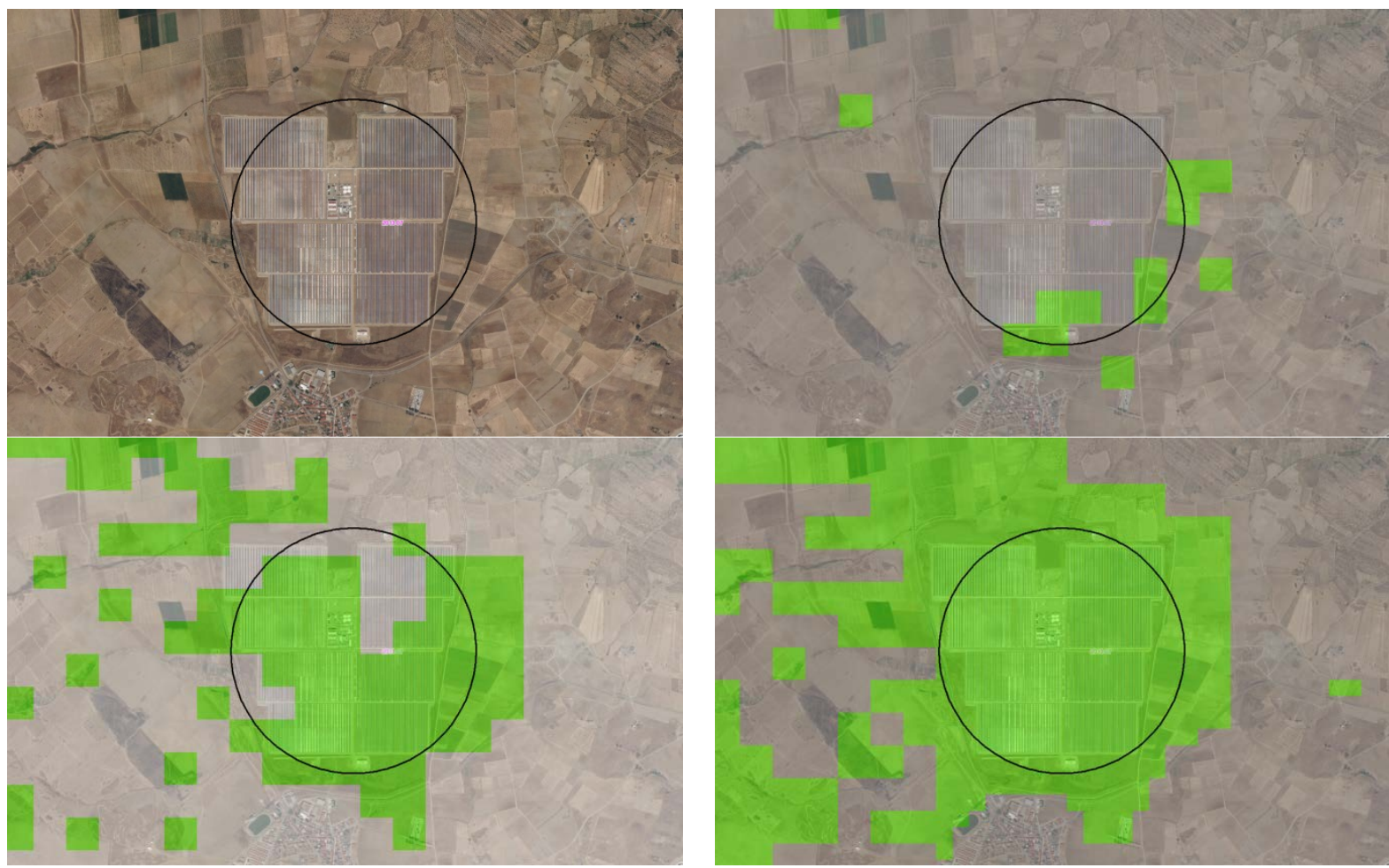

Figure 8-5. Buffer around the Orellana STE plant (Spain) and the suitable areas by the LRS1 (upper right), LRS2 (bottom left), and LRS3 (bottom right) methodologies with maximum slopes of $1 \%, 2 \%$, and $3 \%$. Suitable zones are shown in green (Navarro et al. 2016)

With the introduction of powerful, easy-to-use software tools and web pages, such as Solar Advisor Model (SAM), ${ }^{3}$ Greenius, ${ }^{4}$ RETScreen, ${ }^{5}$ Global Atlas for Renewable Energy, ${ }^{6}$ or the Solar Power Prospector website, ${ }^{7}$ many analysts now expect to use maps and time-dependent modeling of their prospective solar systems as part of the preliminary analysis. Considerable care must be taken to choose the correct irradiance data sets for input to the model. Experts recommend multiple years of at least hourly input data, rather than data from only 1 year or even TMYs, to assess the effects of inter-annual variability of the solar resource on year-toyear system performance. Each hourly data set should be evaluated at least to determine whether the monthly mean values from hourly data match the best estimate of monthly mean values at the proposed site (Meyer et al. 2008). In most cases, the bankability of large projects requires on-site measurements during at least 1 year to validate the long-term modeled time series and correct them if needed using an appropriate site adaptation technique.

\footnotetext{
${ }^{3}$ See www.nrel.gov/analysis/analysis tools tech sol.html.

${ }^{4}$ See http://freegreenius.dlr.de/.

${ }^{5} \mathrm{See}$ http://www.nrcan.gc.ca/energy/software-tools/7465.

${ }_{7}^{6}$ See https://irena.masdar.ac.ae/gallery/\#gallery.

${ }^{7}$ See http://maps.nrel.gov/node/10/.
} 


\subsubsection{Influence of Aerosols}

For solar energy projects, a key step in site screening is to implement a concept called clean air prospecting. This is of special importance for concentrating solar technologies (CST) projects because DNI is more strongly affected by the aerosol optical depth (AOD) than GHI and GTI are. In deserts and other areas with high solar resource, most sites have low annual average cloud cover. Over these areas, the annual average DNI is strongly influenced by AOD. Understanding the AOD characteristics is vital to assessing the solar resource and the performance of CST installations.

AOD is a measure of the optical attenuation effects caused by various types of particles in the atmosphere, collectively called aerosols. These include dust and sand particulates, air pollution, smoke from wildfires and agricultural burning, and sea salt (near coastlines). Over arid or desert areas, the average AOD may be sufficiently low for CST plants even if dust events occur on an infrequent basis. Note, however, that the higher the annual average AOD, the higher its temporal variability (Gueymard 2012), which can be an issue for CST projects in particular.

The analyst should take into account the following questions about the site:

- What are sources of potential aerosols? These may include:

○ Dust storms

- Air pollution

- Fires

- Proximity to urban areas

- Proximity to dirt roads with heavy traffic or to areas where biomass burning is frequent

- Proximity to fossil fuel power plants, mines, etc.

- Does the area have good visibility most of the time? Are distant hills or features visible without the effects of haze?

- No visible haze would indicate that the AOD is indeed low, and, therefore, the irradiance is similar to the modeled map values.

- If the area is known to have some form of visible haze, aerosols are likely to be a problem at the site. Further research or measurements may be necessary.

Typically, areas of higher uncertainty in AOD are coastlines, deserts, or around urban areas. Because such areas may be good candidates for solar energy for economic and infrastructure reasons, additional measurements (of AOD and/or irradiance) may be necessary to resolve whether a site is sufficiently protected from sources of aerosols.

\subsubsection{Volcanic Aerosols in the Stratosphere}

Debris from volcanic eruptions affect the solar resource over large areas (at the country, continental, or global scale) but typically last only while the eruptions last. Very large eruptions, such as El Chichón or Pinatubo, impacted the solar resource globally for up to $\approx 3$ years. The main impact of volcanic aerosols on the solar resource arises when significant amounts of $\mathrm{SO}_{2}$ (sulfur dioxide) are ejected into the stably stratified stratosphere. Smaller volcanic eruptions do not reach the stratosphere and, thus, have short-lived local effects only. $\mathrm{SO}_{2}$ is converted into sulfuric acid aerosol droplets that scatter solar irradiance very 
efficiently. These droplets affect DNI more than GHI due to their optical properties. The droplets can stay in suspension for several years after an eruption. The AOD of the droplets decays exponentially with a decay time of 1-2 years (Crowley and Unterman 2013; Robock 2000).

The last large-scale volcanic eruption was Pinatubo in 1991. Thus, many recent data sets do not cover this. One exception is the NSRDB (Wilcox 2007). Data from this can be used to estimate the effect this eruption had on the solar resource (Vignola et al. 2013). Using ice core data of the long-term past, the probability of a volcanic eruption as strong or stronger than Pinatubo (stratospheric AOD $\geq 0.2$ ) is estimated to be $30 \%$ in the coming decade (Sigl et al. 2015), which is significant.

\subsubsection{Comparison of Modeled Irradiation Resource Data}

During the first stages of a solar project, solar radiation information may be available from different sources, as discussed in Chapter 4 and 5. Having many sources of irradiance data is better than having none, but the question of selecting the best possible source then arises. This may be done through detailed comparisons between them and validation against highquality measurements. Some concepts related to such tasks are stated below. The definitions of variability and error proposed below can help to understand the observed differences among databases better, even though these definitions are not always agreed upon by all analysts or applicable to all possible applications. In addition to these concepts, detailed discussions on uncertainty definition, characterization, and calculation are provided in Chapter 6.

Variability: Expected or actual dispersion of a variable during a specific period (temporal variability) or over a specific area (spatial variability). It can be expressed as the coefficient of variation (COV) for variables having a normal distribution, as the variance for any other known statistical distribution, or as the interquartile range when the distribution is unknown. $\mathrm{COV}$ is obtained by dividing the standard deviation by the mean of the population or sample.

Variability relates to the analyzed time period (e.g., yearly variability of daily GHI; long-term variability of DNI) or to a given geographic area (e.g., spatial variability of DNI over an area of $50 \times 50 \mathrm{~km})$.

Error: Difference (or deviation) between a measured or estimated value vs. the value of the measurand/quantity. The latter value has to be measured or estimated by an adequate procedure using specialized and well-maintained instruments, harmonized protocols, or international standards. The individual error at instant $i$ can be expressed as

$$
e_{y_{i}}=y_{i}-x_{i}
$$

where $e_{y_{i}}$ is the error of the estimate $y_{i}$, and $x_{i}$ is the value of the quantity.

Common error expressions are mean bias error (MBE or BIAS), mean absolute error (MAE), mean square error (MSE), and root mean square error (RMSE). When the most probable value is actually uncertain itself (which is the most general case), the error should rather be referred to as a difference or deviation; then letter $\mathrm{E}$ is replaced by $\mathrm{D}$ without any change in the calculation (Gueymard 2014).

Typically, some individual errors or differences are of higher magnitude than uncertainties. In general, the accuracy of modeled data is reported in terms of conventional error statistics. In 
addition, Espinar et al. (2009) proposed a new statistical indicator, called KSI (KolmogorovSmirnov Integral). It evaluates the area between the distribution functions from the two tested samples. Gueymard (2014) reviewed this and many other statistical indicators for solar radiation series comparison and applied them to the performance evaluation of a variety of clear-sky radiation models.

A study conducted by the Management and Exploitation of Solar Resource Knowledge (MESoR) project in Europe (Beyer et al. 2008) provided insights into the spatial distribution of irradiance variability by cross-comparing five different data sources. Inherent differences were found between databases based on in-situ (ground) measurement interpolations and those based on satellite observations, as well as in the methods used to process such data. Those databases relying on the interpolation of ground observations were sensitive to the quality and completeness of ground measurements and to the density of the measurement network. Terrain effects (e.g., shadowing by surrounding terrain) played a role in solar radiation modeling over hilly and mountainous regions. The spatial resolution of the input data and the selected digital elevation model (DEM) were identified as factors with direct impact on the accuracy of the estimates. Finally, in order to compare modeled data properly, it is important to take into account how each model deals with cloud identification and AOD characterization (Ruiz-Arias et al. 2016). This is particularly important in the case of DNI due to its higher sensitivity to AOD than GHI.

The quality and spatial detail of satellite-derived or numerical databases are determined by the specific input data used in the models. As can be expected, the main parameters are those describing the cloud properties and then those describing the optical state of the atmosphere, such as the atmospheric turbidity due to aerosols or water vapor (Ineichen and Perez 2002; Ruiz-Arias et al. 2016). Regarding DNI more specifically, AOD is the most important variable under clear-sky conditions (Gueymard and George 2005). Cebecauer et al. (2011) provided a comprehensive and qualitative review of the different factors (including terrain) affecting the accuracy of DNI modeling.

The studies conducted so far provide only a preliminary outline of the state of the art of current knowledge in irradiance modeling. These studies still do not fully address the needs of the solar energy industry, so further work is needed to improve knowledge and decrease the uncertainties. In most cases, similar studies must be performed for the sites of interest within an individual project.

\subsubsection{Variability of the Solar Resource}

Predicting the behavior of existing or future solar systems assumes that the temporal and spatial irradiance variability can be adequately characterized with measurements and/or modeled data. It is easy to take care of the deterministic variability due to location, date, and time of day. What matters most is the variability (temporal or spatial) due to that in weather and climate.

With some knowledge about the inter-annual irradiance variability at a specific site, users can in principle select a particular experimental period to adequately characterize the solar resource. Although, ideally, such on-site measurement campaigns should last many years, practical reasons limit them to 1 year or less in the majority of cases, which increases the uncertainty in the long-term estimates. Likewise, with knowledge of the spatial variability over the area around a measurement station, users can evaluate the applicability of those measurements to a location some distance away. Knowledge of variability then becomes valuable when deciding how long to make measurements at a particular location and whether 
the characteristics of the solar resource at that location can be extrapolated to other nearby locations.

Gueymard and Wilcox (2011) analyzed 8 years of data (1998-2005) from the U.S. NSRDB in the realms of temporal and spatial variability. Results provided the annual spatial and temporal variability of DNI, GHI, and GTI over the continental United States. The analysis of temporal variability summarized the COV values in each $10-\mathrm{km}$ by $10-\mathrm{km}$ cell in the State University of New York (SUNY) satellite-derived data constituting the version of the NSRDB then available. The COV is the standard deviation of a set of values divided by their average. As could be expected, the variability of DNI is larger than that of GHI for a given location. Interestingly, the temporal DNI variability was found to vary largely over the United States and even within a single state. For instance, COV varied from a low of $0.5 \%$ in south-central Washington to a high of $15.8 \%$ in northwest Washington, as shown in Figure 8-6. This was explained by the high humidity and cloudiness in the first case and the much dryer and clearer conditions in the second. For the case of spatial variability, square matrices of several cells were analyzed. Two matrix sizes, $3 \times 3$ and $5 \times 5$, were analyzed, using the annual average maps of DNI, GHI, and GTI for the whole period. These matrices represent areas of approximately $30 \mathrm{~km}$ by $30 \mathrm{~km}$ and $50 \mathrm{~km}$ by $50 \mathrm{~km}$, respectively, and likewise roughly represent an area within $15 \mathrm{~km}$ and $25 \mathrm{~km}$ of a measurement site, respectively. COV was found to vary between $0.1 \%$ in central Missouri to $\approx 11.5 \%$ along a corridor between Los Angeles and San Bernardino, California. Variability tends to be higher in coastal areas and in mountainous areas. Greater variability occurs in the 5-by-5 matrix, which is to be expected because of the effects of terrain. Furthermore, the general pattern of high and low variability remains the same between the two matrix sizes, indicating that at locations of significant variability, most of it occurs over short distances.

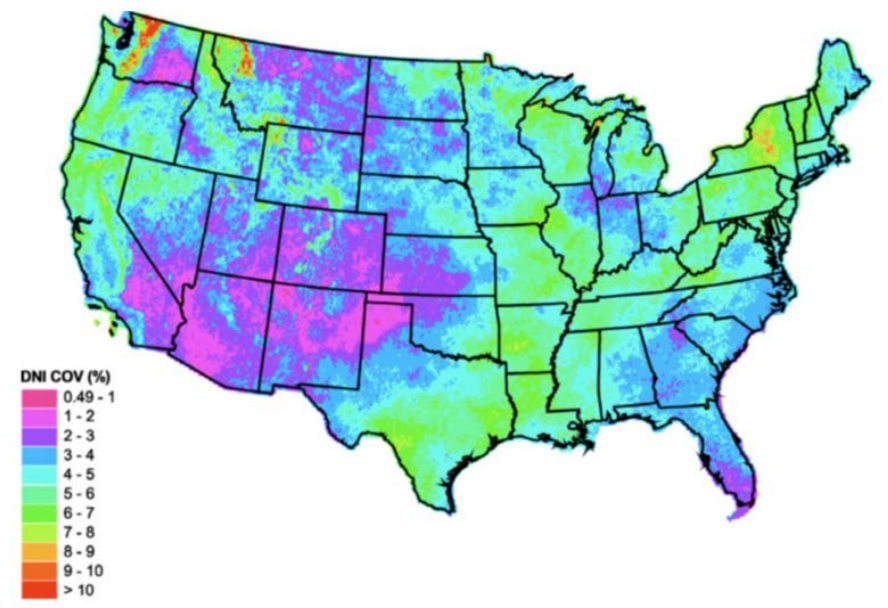

Figure 8-6. Inter-annual DNI variability (COV as percent) for 1998-2005 (Gueymard and Wilcox 2011)

Using these variability statistics, users can better understand the extent of measurements required to best characterize the solar resource for a particular application. In areas with low inter-annual variability, a shorter measurement period may suffice. In areas with low spatial variability, a measurement station could possibly represent the solar resource at nearby locations (e.g., within 10-50 km), avoiding the need for additional measurements. An analyst can use this information to better build confidence in a data set as being sufficient for an analysis and can use these data to understand the consistency of future solar power plant performance and how that relates to the economic viability of a particular location. 
Similar analysis was carried out for a longer gridded data set (1998-2009) and its corresponding TMY (Habte et al. 2014). The spatial variability analysis was implemented by comparing a center pixel to neighboring pixels. A higher variation in the DNI compared to the GHI was attributed to the opacity of sky and also aerosols (Gueymard and Wilcox 2011). The magnitude of spatial variation in DNI increased rapidly as the distance between the center pixel and the farthest pixel increased, as could be expected from the previous study. The variation of DNI with adjacent pixels can thus provide system performance analysts with essential information about how the system energy output tends to be spatially variable in certain locations. The temporal variability was also analyzed using a standard deviation for the monthly gridded NSRDB (1998-2009) data.

Fernández-Peruchena et al. (2016) tested the assumption of normal distribution in annual GHI and in DNI values, which was assumed by Cebecauer and Suri (2015). Results from the Kolmogorov-Smirnov (KS) and Shapiro-Wilk normality tests indicate that the assumption that annual GHI values follow a normal distribution cannot be rejected at all 10 tested locations. In the case of DNI, five tests were applied to the annual DNI series for evaluating the Weibull goodness of fit at six locations. Results weakly reject the null hypothesis. Moreover, the normality tests applied to the same annual DNI data provide rejection of the null hypothesis only at two of the six tested locations.

Taking into account all these results, the temporal variability has to be further analyzed in order to be able to clarify whether annual, monthly, or seasonal solar radiation values can be properly assumed independent and just random samples of the same population or, conversely, represent different probabilistic models having, for example, a stationary behavior.

\subsection{Data Applications for Feasibility Assessments}

After the analyst has selected one or more candidate sites for an engineering feasibility assessment, the next problem facing solar power plant project developers is to obtain data sets that can guarantee the most reliable calculation of annual or inter-annual system performance. There are different possible situations depending on the availability of shortterm and/or long-term measured data sets and/or of other modeled data sources. Usually, at least one whole year of local measurements is required to guarantee the bankability of large solar energy projects (Ramírez et al. 2012).

Solar system simulations typically use two different types of irradiance time series: (1) an annual meteorological data set related to the mean expected year-usually referred to as TMY or typical reference year (TRY); or (2) annual meteorological data corresponding to a year with a low irradiance in order to test the project's revenue and financial stress under quasi "worst-case scenario" conditions. The following subsections provide a review of the current methodologies and possible improvements for the generation of these two types of data sets. Section 8.3.3 provides a view of post-processing and site adaptation methodologies for reducing uncertainty in the data sets used for generating the series for simulation.

\subsubsection{Typical Meteorological Data for Solar Energy System Simulations}

Typical meteorological data sets with hourly (or sub-hourly) resolution are used as the standard input to a wide range of solar energy system simulation software. Such a data set is specifically constructed to provide analysts with a compact annual data set normally holding 8,760 hourly meteorological values that typify conditions at a specific location throughout a longer period from 10-30 years. 
The first yearly data sets were the standard year (Benseman and Cook 1969), the TRY (Andersen et al. 1974; Crawley 1998; Lund 1974), and the TMY (Hall et al. 1978). The Hall method was used by NREL after the completion of the first NSRDB (1961-1990) in the mid1990s (Marion and Urban 1995). The NSRDB(1961-1990) represented a 30-year hourly data set of measured and (mostly) modeled ground-based solar radiation elements for 239 weather stations located throughout the United States, and the TMY data set generated from the NSRDB(1961-1990) was branded as TMY2 to distinguish it from the Hall et al. (1978) version. Later, when the NSRDB was updated, some further refinements were also made to the TMY2 data set, and the updated data set was branded as TMY3 (Wilcox and Marion 2008). TMY3 was generated using NSRDB (1961 - 1990) and NSRDB (1991 - 2005). Any available type of TMY is widely used throughout the solar community, in addition to the building community. In all TMY construction methods, 12 typical meteorological months (TMMs) are selected by finding the specific year for which the cumulative frequency distribution of selected weather elements (including irradiance and temperature) is closest to that of the mean or median for that month. The longer-term distributions are determined for each month using data from the full period of record. The TMMs are then concatenated, essentially without modification, to form a single year with a serially complete data record. The resulting yearly data set may contain measured or modeled time series of solar radiation and surface meteorological data, although some hourly records may contain filled or interpolated data for incomplete periods.

TMY and TRY data sets were originally designed for simplified building energy simulations and thus contain a number of weather variables that are important for that application but not necessarily for solar applications. TMYs were also suitable for the limited computing resources available at the time. Today, if long-term meteorological time series that include high-quality solar irradiance measurements are available for a location, these should be used instead.

TMY and TRY data sets are still widely used by building designers and solar engineers for rough modeling of renewable energy conversion systems and their preliminary design. Although not designed to provide meteorological extremes, these data have natural diurnal and seasonal variations and represent a year of typical climatic conditions for a location. TMYs should not be used to predict weather or solar resources for a particular period of time, for preparing the project's final design, or for evaluating real-time energy production. Because a TMY represents "typical" (or more precisely, median) conditions over a long period, such as 30 years, it is not suited to analyze the system's response to worst-case weather conditions that could occur in the future. For that reason, it is unfortunate that many software applications are designed to exclusively use TMY data to predict the typical performance of a solar conversion system, which may lead to various issues.

What distinguishes the different yearly data sets is how the TMMs are chosen. Different meteorological variables may be considered important and weighted accordingly in the selection process. For solar heating and cooling (SHC) purposes, GHI, temperature, and relative humidity are of primary importance and are the basis for the TMM selection for design reference years (DRY) (Lund 1995; Skartveit et al. 1994) and energy reference years (ERY) (ISO 15927-4 2005). In the NREL TMY data sets, wind speed and DNI are also considered in the selection. The representative solar year (RSY) (Ramírez et al. 2012) format is specifically designed for STE projects, for which DNI is the primary meteorological variable. 
There are some available products of gridded satellite-derived data sets, such as typical direct normal irradiance year (TDY) and typical global horizontal irradiance year (TGY), that are exclusively selected from DNI and GHI data, respectively, per the NREL method (Habte et al. 2014) or the SolarGIS method (Cebecauer and Suri 2015). A review of methodologies for creating reduced meteorological data sets is provided by Nielsen et al. (2017). Some standardization bodies have promulgated standards in an attempt to harmonize all the existing methodologies. For instance, ISO 15927-4 (2005) focused on the hydrothermal performance of buildings for assessing the annual energy use for heating and cooling. A Spanish standard (AENOR 2014) targets STE power plants and constitutes the first draft for the final IEC Technical Commission TC-117 Technical Specification (TS) 62862-1-2.

To prepare TMYs, the underlying long-term data series are rarely provided by local measurements due to their paucity. Most generally, TMYs are prepared from modeled data, which typically include some bias in the irradiance data. To avoid this issue, the developer must perform a site adaptation of the long-term modeled data by applying appropriate fitting methodologies to local measurements, as mentioned above and described further in Section 8.3.3. The AENOR (2014) standard proposes an additional way to obtain annual data series, using a whole year of valid on-site measurements as the input data for the series generation. Once the values of the TMMs are found using the long-term fitted estimations, days are changed in each month to achieve the TMM value for that month. The methodology defines the limited number of changes, the limited number of repetitions, and the maximum distance for a day's substitutions. The use of this type of series aims to avoid fitting problems in the daily profiles and the need of increasing the temporal resolution of some data sets. Even more refinements are proposed by Lara-Fanego et al. (2016).

\subsubsection{Inter-Annual Variability and Probabilities of Exceedance}

In the case of large solar energy projects, bankability requirements are stringent; hence, reliable profitability and annual payback assessments have to be performed. Thus, probabilistic information about the energy output is needed. This must be based on probabilistic solar resource time series that correctly account for extreme situations, which obviously require the statistical examination of long-term time series.

A preliminary step is to first determine the minimum period during which ground measurements should be taken at a proposed site before the true long-term mean is captured because this is the most critical characteristic of the solar resource. Another way to look at the problem is to ask how representative a 1-year average is to the "true" climatological (nominally 30 years) mean. In the wind industry, a rule of thumb is that it takes 10 years of on-site wind measurements to obtain a mean annual wind speed that is within $\pm 10 \%$ of the true long-term mean, which is generally required by financial institutions. But in a case with only 1 or 2 years of on-site measurements, these data may be all that is available to a financial institution conducting due diligence on a project.

Tomson et al. (2008) showed that the mean annual GHI in any year is virtually independent from that of the previous year, which implies that even many years of on-site measurements may not represent the long-term mean. Gueymard and Wilcox (2009) analyzed the long-term data from four stations with continuous high-quality measurements over $\approx 25$ years to examine how many individual years would be required to converge to the long-term mean and whether the inter-annual irradiance variability changes significantly from one site to another. Sorting the data from the worst years (largest anomalies) to the best years (smallest anomalies), the results show (Figure 8-7) that first, there is much lower inter-annual 
variability in GHI than in DNI. GHI is almost always within $\pm 5 \%$ of the true long-term mean after only 1 year of measurement. The situation is quite different for DNI. After only 1 year of measurements, the study shows that the estimate of the average DNI is no better than $\pm 10 \%- \pm 20 \%$ of the true long-term mean. At two of the sites, more than 10 years of measurements are required to be within $\pm 5 \%$ of the true long-term DNI mean, which is consistent with the findings in the wind energy industry. Note, however, that the worst years were associated with strong volcanic activity, which significantly impacts DNI.
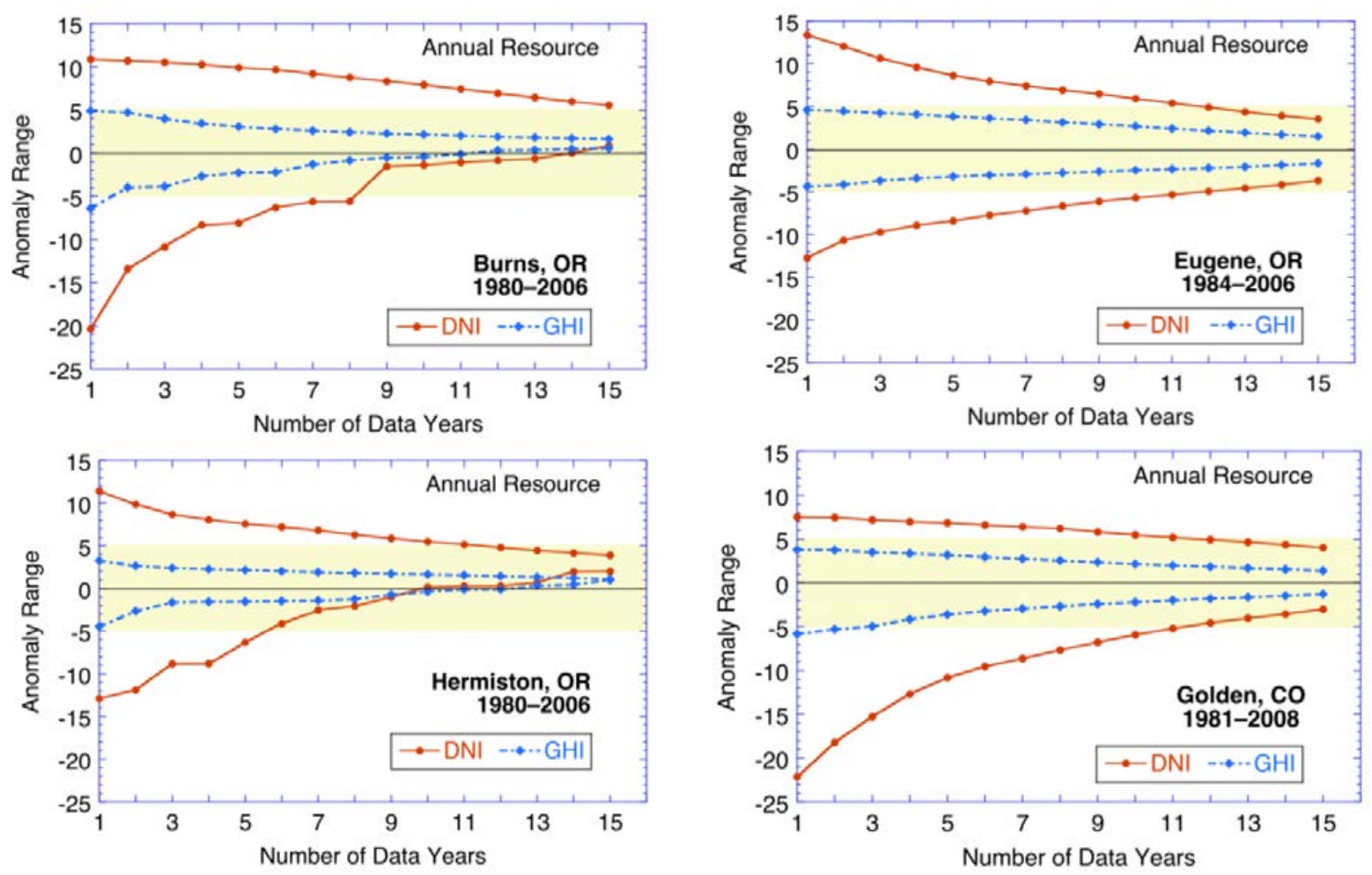

Figure 8-7. Number of years to stabilize DNI and GHI at (clockwise from upper left) Burns, Oregon; Eugene, Oregon; Hermiston, Oregon; and Golden, Colorado. Specific sorting from the worst years (largest anomalies) to the best years (smallest anomalies)

(Gueymard and Wilcox 2009)

Because long-term on-site measurements are the exception more than the rule, these results underline the importance of relying on an independent long-term data set, which, in practice, means a satellite-derived data set. This is necessary to reduce the uncertainty in the long-term average DNI estimates for a proposed STE site and provide reasonable due diligence of a plant's estimated performance throughout the life of the project. This and additional concepts related to the development of specialized TMYs or annual series for energy simulation are described by Vignola et al. (2012).

Financial institutions evaluate the risk of uncertainty in solar resource data through the probability of exceedance (POE). POE, which is also denoted by "P," is the complementary value of a percentile value. In the case of POE50, its value matches the $50^{\text {th }}$ percentile and is the result of achieving an annual energy production based on the long-term median resource value. For this value, the probability of reaching a higher energy value is $50 \%$. For example, TMYs represent the P50 value. In contrast, for POE90, the risk that an annual energy value is not reached is $10 \%(90 \%$ of all values in a distribution exceed the POE90 value). The POE90 corresponds to the $10^{\text {th }}$ percentile. Often, the notation P90 is used instead of the clearer term POE90. Care must be taken because POE90 is understood in some cases as the $90^{\text {th }}$ 
percentile, and hence, using the notation POE90 is recommended. Depending on a project's size and financial institution, the solar resource's "bad years" may be examined using various POEs, from lax (POE75) to stringent (POE90, 95 or even 99). In addition, these estimates are examined in relation with the uncertainty in the data. High uncertainty is always an issue, even if the POE results appear favorable. The combination of probabilistic performance modeling and uncertainty inherent to various components of the system (including the solar resource) requires specialized developments (Ho et al. 2011; Ho and Kolb 2010).

The effect of reducing the uncertainty of the true long-term solar resource at a site in order to reduce financial risk is demonstrated in Figure 8-8, taken from a study by Moody's Investors Services (2010) (see the discussion in Renné 2016). Figure 8-8 shows that with only 1 year of data, the uncertainty in both the value and the distribution of the true long-term mean is much higher than with 10 years of data. Thus, assuming that a long-term annual data set follows a Gaussian or normal distribution (which is not necessarily the case), the standard deviation that is assigned to a 1-year data set is much higher than for a 10-year data set because there is more information associated with the 10-year data set. Although the median value of the distribution does not change between the 1-year and the 10-year distribution curves, the POE90 value increases with the additional knowledge (higher confidence) associated with having a 10-year data set, which would lower the financial risk of the project. The uncertainty in the irradiance estimates (from modeling or measurement) is also considered to ultimately evaluate the uncertainty in the PoE and correct it if necessary.

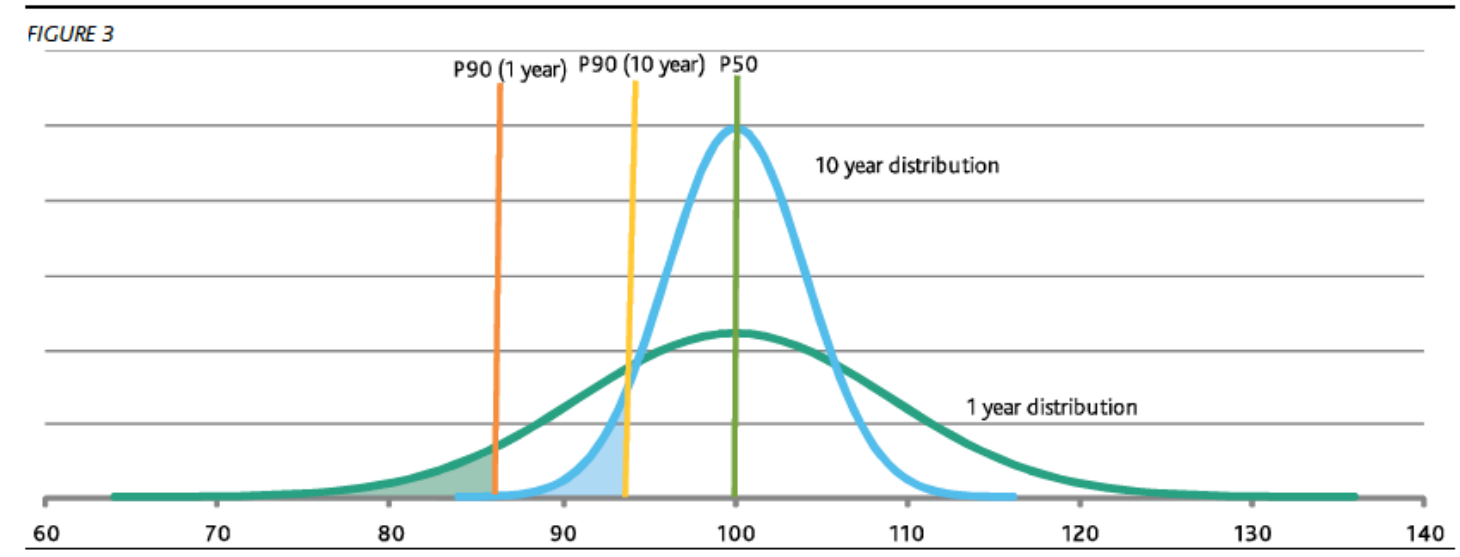

Figure 8-8. With only 1 year of data, the uncertainty of the value of the true long-term mean is much higher than with 10 years of data (Moody's Investors Services 2010)

The statistical calculations above assume that long-term irradiance data follow a normal (or Gaussian) distribution. This assumption, however, may not be correct. For example, Dobos et al. (2012) have looked at the NSRDB GHI and DNI data for Phoenix, Arizona, and produced cumulative distribution functions (CDFs) based on 30 separate annual data sets to illustrate the concept of P50 and POE90. Figure 8-9 shows that if the annual Phoenix data were fit to a normal distribution (solid line) at $\mathrm{CDF}=0.1$ (which corresponds to the POE90 value), an annual GHI of $1.96 \mathrm{MWh} / \mathrm{m}^{2}$ would be exceeded $90 \%$ of the years (or conversely, the solar resource would fall below this value $10 \%$ of the years). Similarly, for DNI, the annual solar resource exceeds $2.2 \mathrm{MWh} / \mathrm{m}^{2} 90 \%$ of the years. However, for Phoenix the long-term solar data does not follow a normal distribution, and Figure 8-9 shows that the POE90 value is somewhat lower in Phoenix when determined from an empirical vs. a normal distribution. Further discussion on these points can be found in Renné $(2017,2016)$. 

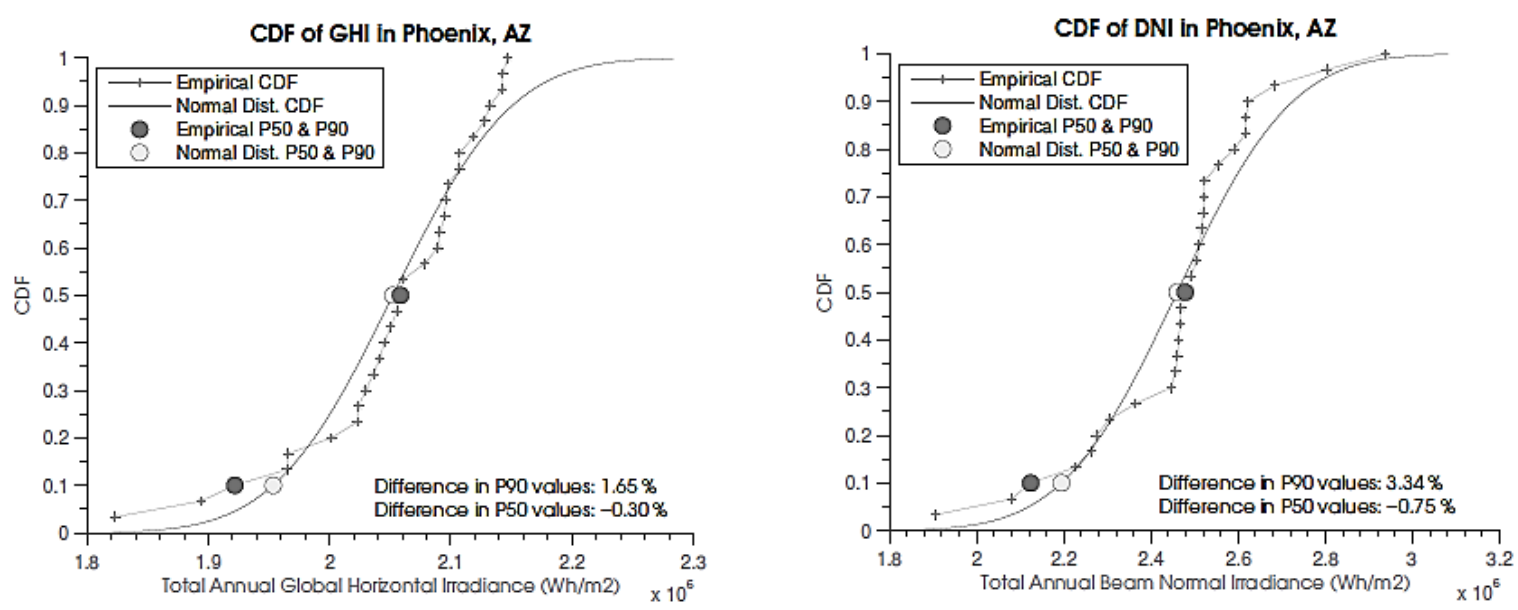

Figure 8-9. (Left) Annual GHI data fitted to a normal distribution (solid line) for Phoenix, Arizona. (Right) Same as left plot but for DNI. Note that each gray circle covers a marker (+).

As discussed by Pavón et al. (2016) and Ramírez et al. (2017), there are several issues related to a POE estimate. The first one is the assumption that, for instance, an irradiance at the POE90 level is proportional to the POE90 of the produced energy output, or "yield," which actually constitutes an approximation only. Additional elements are thus needed to: (1) identify the most appropriate POE value; and (2) construct a specific time series for that POE using hourly or sub-hourly data during a year, whose sum is that specified POE value. A statistically based estimation of the POE value depends on the assumed probability distribution. This probability distribution may be approximated with the normal distribution in the case of annual GHI, but for DNI there is no evidence that a normal, a log-normal, or a Weibull distribution would always be the best choice. When 10 , or preferably more than 20 , whole years of local measurements or modeled estimates are available, methodologies based on the cumulative distribution function should be used, such as that proposed by Peruchena et al. (2016). In addition, whereas conventional TMYs only provide median (POE50) resource information, new techniques are developed to construct meteorological years for bankability scenarios that correspond to the POE90, for example (Cebecauer and Suri 2015; Lara-Fanego et al. 2016).

An additional issue is the resolution of the data time series used for energy simulations. For STE projects, for instance, the yield and probabilistic predictions obtained with hourly data may differ substantially from those using 1-minute or 5-minute data (Hirsch et al. 2010; Meybodi et al. 2017). Satellite-derived irradiance time series are typically not available at a temporal resolution better than 15 minutes. Some stochastic methods have been proposed to derive 1-minute or 5-minute irradiance from data at coarser resolution (Grantham et al. 2017; Hofmann et al. 2014).

Instead of using a limited number of yearly data sets for simulation, the use of Monte Carlo methodologies to generate an unlimited number of yearly series is proposed by Nielsen et al. (2017). This methodology allows the solar resource assessment, and thus the energy output calculation, to be performed in a similar way as what is currently used for estimating other essential variables in the economic assessment of solar power plants. The generation of hundreds of such plausible years has been demonstrated by Fernández-Peruchena et al. (2015) and Meybodi et al. (2017). Other authors (Ho et al. 2011; Ho and Kolb 2010) found issues with the Monte Carlo approach and rather suggest the Latin hypercube sampling method. 
It is worth mentioning that including long-term trends, or even International Panel of Climate Change (IPCC) scenarios (IPCC 2000), may improve the accuracy of the solar plant yield prediction (i.e., during the complete solar facility lifetime). This is offered by the

Meteonorm ${ }^{8}$ software, for instance. Aerosol pollution scenarios are also important for the future GHI and DNI resource. For instance, a decrease of the solar resource has occurred in many Asian countries during the recent past, and this trend may continue in the foreseeable future.

\subsubsection{Combining Data Sets and Site Adaptation}

A solar energy project relies on solar resource data. Long-term input data sets always have uncertainty. If its magnitude can be precisely evaluated, investors can derive the risk of the project and evaluate whether the performance of the system could be lower than desired. Reducing uncertainty in solar resource data is thus a key step toward producing bankable projects.

Sometimes combining data sets and site adaptation are called post-processing techniques (Janotte et al. 2017). These cover a wide variety of methodologies that are applied to improve direct model or retrieval outputs and reducing uncertainty. Some studies propose a chain of methodologies (Blanc et al. 2012) that ends with the final site adaptation (Figure 8-10). All the post-processes try to improve the model behavior using the long-term direct model output and simultaneous, additional data from, for example, a shorter period or a lower spatial resolution and look for a relationship. After that, the relationship is applied to a longer period or a higher spatial resolution of the model output.

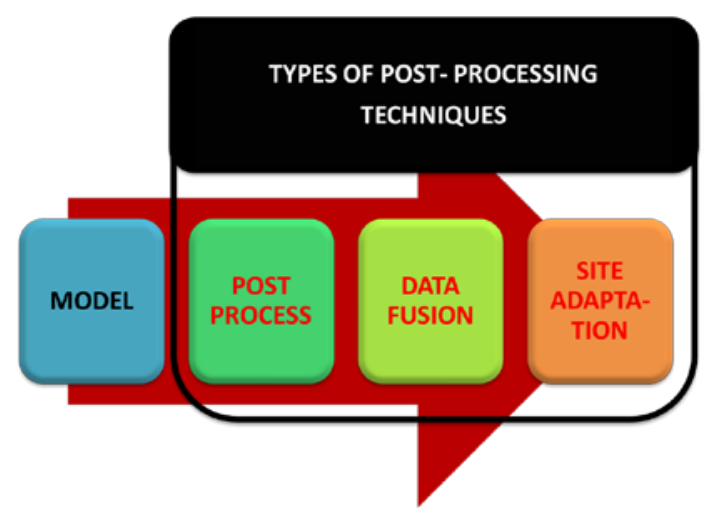

Figure 8-10. Added value and accuracy chain for solar radiation model output (Janotte et al. 2017)

Two main post-processing cases can be distinguished: (1) regional adaptation or (2) local adaptation. Regional adaptation is performed by model developers using available related modeled variables, as well as all available ground measurements in the geographical area covered by the specific product data set. Examples of regional post-processing are reported frequently by model developers (e.g., Bernardos et al. 2015; Ruiz-Arias et al. 2015). Local adaptations are performed by experts using the outcome from a data set from a single location (possibly subjected to a preliminary regional adaptation) and local ground measurements. These local ground measurements are commonly not available for the product development and must be collected for the specific project developer. This assessment focuses on the specific location and manages as many databases as possible as well as at least 1 year of valid

\footnotetext{
${ }^{8}$ See http://www.meteonorm.com/.
} 
local measurements (Ramírez et al. 2012). Various techniques are possible, with varied degrees of sophistication, including methods derived from the post-processing of meteorological forecasts based on NWP modeling (Gueymard et al. 2012; Polo et al. 2016). The principle of some of the simplest methods is summarized in what follows.

The most straightforward method is the ratio estimation. In this method, the ratio of a selected period of concurrent independent data sets (a short-term measurement data set concurrent with the long-term modeled data set) is calculated, and then the ratio is applied to the remaining long-term data set (Gueymard and Wilcox 2009). However, the uncertainty in this approach increases if the biases in either data set vary over time. Furthermore, if there are long-term trends in the data, a longer measured data set is required.

One basic approach for combining data sets from various independent sources uses a weighting criteria (Meyer et al. 2008). The weighting factors can be determined based on known data uncertainties. Although this method was designed primarily for merging modeled data sets, measured data sets can also be included if their uncertainties are known. This approach uses all available data for each time period, but short-term data do not have impact on long-term data and vice versa.

Another approach for site adaptation is to use the CDF of ground-based data to improve satellite-derived data (Schumann et al. 2011). This approach reduces bias errors and improves the satellite data's KSI. Mieslinger et al. (2014) have recently improved this approach by determining both the systematic and random deviations of the data sets based on mean bias (for systematic) and standard deviation (for random) statistics and using the KSIs to quantify the differences in the CDFs. Polynomial expressions are then used for the site adaptation. This approach is especially useful when large deviations between the satellite-derived and the ground-measured data exist. The approach requires at least 1 year of overlapping data, but preliminary results show that the corresponding biases approach zero.

The quality of the solar resource data used in a solar project ultimately determines (to a large extent) its production uncertainty and, therefore, the overall project risk (Renné 2016). The following two options are recommended:

- Collect high-quality on-site ground data for many years. This is the best way to provide the lowest risk data set to a financial organization; however, it is generally impractical due to the time required to collect sufficient data (e.g., 2 years for GHI or 10 years for DNI).

- Combine long-term modeled (e.g., satellite-derived) data with short-term high-quality on-site measured data. This approach, known as site adaptation, is the most practical and cost-effective approach for developing reliable solar data for a project and offers the lowest risk to developers without causing lengthy project delays. First, collect high-quality on-site ground data for a short time (1 year or less). This is more practical approach compared to long-term on-site measurements with respect to investor time scales and, thus, has become a quasi-standard procedure. However, the additional long-term data are needed because with the short-term measurement alone, one obtains no knowledge of inter-annual variability and solar resource trends, even if, by chance, the measurement period is representative of the long-term solar resource. Next, multi-year satellite-derived or other model-derived data sets are obtained. In the previous sections, it was noted that ancillary weather data, such as weather satellite imagery or ground observations of cloud cover, can be used to 
produce long-term data sets wherever measurements are not available (which is the typical case). Combination of several of these model-derived data sets can potentially lead to a reduction of uncertainty. For investors, however, the risk of using only modeled data is higher than if actual high-quality measured data for the same time period were available. Therefore, methods are applied to combine the available data sets. As mentioned above, Polo et al. (2016) provide a comprehensive review of these methods.

\subsection{Solar Radiation Needs During Plant Construction: Yield Estimation}

This section provides a summary of general approaches of using solar resource data (as described in Chapters 1-5) to estimate the yield of solar energy systems. First, PV systems are discussed, followed by concentrating systems. This section incorporates some excerpts from the previous version of this handbook (Sengupta et al. 2015) due to their long-lasting interest.

In the case of STE, the conversion from concentrated light into electricity involves a solar receiver model, the transfer of the heat to the turbine, and the storage and the operation of the turbine. For CPV, the module performance, the inverters, and, if present, a battery system, must be modeled.

\subsubsection{Yield Estimation of Non-Concentrating PV Projects}

The value of electricity generated by a PV plant depends on the amount of electricity generated and on the grid's need for that electricity at the time it is generated (i.e., its load curve). A quantitative understanding of the specific solar resource for the intended location and orientation of the PV array is essential to evaluate the first quantity. The relevant solar input for yield calculation is the irradiance incident on the plane of array (POA) (i.e., GTI), although other parameters (particularly ambient temperature, wind, and soiling) also impact the system's output. This section provides only a high-level overview; more detailed descriptions of PV system modeling can be found in Ellis et al. (2011). Three general approaches exist to estimating a PV system's yield. These are presented in order of increasing accuracy.

\subsubsection{Performance Ratio Method}

The output of a PV plant can be characterized by the performance ratio (PR) metric, which describes the fraction of electricity generated by the plant relative to what the plant would generate if it always operated at its nameplate efficiency (International Electrotechnical Commission 1998). Typically, new PV plants operate with performance ratios of $0.8 \pm 0.1$ (van Sark et al. 2012). Thus, if the annual solar resource available for a given site and given array orientation (fixed or tracking) is determined, the annual output of the system can be estimated according to the following equation:

Annual output $(\mathrm{kWh})=\mathrm{PR} *$ solar resource $\left(\mathrm{kWh} / \mathrm{m}^{2}\right) * \mathrm{PV}$ plant size $\left(\mathrm{m}^{2}\right)$ 2)

or

Annual output $(\mathrm{kWh})=\mathrm{PR} *$ normalized solar resource $(\mathrm{kWh} / \mathrm{kW}) * \mathrm{PV}$ plant size $(\mathrm{kW}) \quad(8-3)$ 
where the PV plant size is derived from the sum of each module nameplate rating, which is obtained under standard test conditions $\left(\mathrm{STC} ; 1 \mathrm{~kW} / \mathrm{m}^{2}\right.$ ). Deviations from this estimate can be expected due to the inter-annual variability of the solar resource and the variability of the performance ratio (van Sark et al. 2012). Contributors to low performance ratios include

- Shading losses

- Soiling or snow-coverage losses

- High-temperature operation

- Undersized inverters, making them "clip" the plant output part of the time

- Older plants that have experienced degradation

- Modules that have below nameplate performance (today, many manufacturers bin modules so that the performance is equal to or greater than the nameplate value, whereas in past years, manufacturers often placed modules in the bin with the larger nameplate value).

Contributors to high performance ratios include:

- Operation in a cold climate

- Modules with low-temperature coefficients (typically, CdTe and high-efficiency silicon modules tend to have the lowest temperature coefficients)

- Modules that generate power well above the nameplate rating.

The performance ratio method is simple but may not be accurate in all cases. For instance, van Sark et al. (2012) found a few older PV systems with performance ratios less than 50\%. The method is particularly useful, however, to compare the performance of existing systems or to quickly use solar resource data that may not be available to the alternate performance models presented in the next sections. Otherwise, using a more sophisticated performance model is most likely the better approach.

\subsubsection{Simple PV Performance Models}

Among other simple models, NREL's PVWatts or photovoltaic geographical information system (PVGIS) are free online tools that estimate the electric energy production of roof- or ground-mounted PV systems based on a few simple inputs. ${ }^{9,}{ }^{10}$ The user enters a street address or geographic coordinates of the system's location and specifies the system size, array orientation, and the module technology type. The two example tools also allow the modeling of tracked PV systems. The tools then calculate estimated values for the system's annual energy incident on the installation and the electricity production. In the case of PVWatts, hourly energy production and the monetary value of the produced electricity is calculated. For the latter, the user provides some information about the system's cost and electricity rates.

By default, PVWatts uses the TMY2 data set for locations in the United States. Other solar resource data options are available for world locations, but in most cases, some spatial extrapolation is implied. Advanced users may change default assumptions for losses caused

\footnotetext{
${ }^{9}$ See http://pvwatts.nrel.gov.

${ }^{10} \mathrm{See}$ http://re.jrc.ec.europa.eu/pvgis/.
} 
by shading, soiling, and other factors. Full details about the underlying PVWatts algorithms can be found in Dobos (2014).

PVGIS provides a geographical assessment of solar resource and performance of photovoltaic technology. PVGIS was originally developed for Europe but now has been extended to Africa and Asia.

In summary, such tools provide a very convenient and more accurate analysis method than the performance ratio described in the previous section, so it is recommended any time a quick estimate is needed.

\subsubsection{Detailed PV System Performance Models}

More accurate estimates of PV system performance can be obtained by setting up a detailed model of the PV plant that includes choosing specific modules and inverters, an array layout, detailed losses, and shading analysis. Examples of freely available programs that include such detailed performance models are SAM,${ }^{11}$ RETScreen, ${ }^{12}$ and Greenius ${ }^{13}$ (Quaschning et al. 2001). Other commercial programs exist, such as archelios ${ }^{\mathrm{TM}}, \mathrm{PV} * \mathrm{SOL}$, or PVSYST. As an example, the software SAM is introduced below.

SAM is a desktop software developed by NREL that enables the calculation of both detailed system performance and economics for a PV plant (Blair et al. 2014). It integrates several detailed models and databases of thousands of commercially available components that are used to accurately predict the performance of specific PV modules and inverters and their combinations in an array. SAM supports complex system designs that may have multiple subarrays and estimations of shading losses for systems arranged in regularly spaced rows because of their irregular obstructions and detailed accounting of other losses in the system. SAM's PV model calculates energy production for each hour of the year using the user's selection of any solar resource data file. The accuracy of the estimation of annual yield from the PV plant is still dependent on the uncertainty and variability of the solar resource, but the more sophisticated component and system-level algorithms can more accurately model the response of a PV plant to changing meteorological conditions. The accuracy of the model is often dependent on the user's ability to estimate losses from soiling and/or snow. To validate its estimates, SAM has been compared to measured power plant performance data from numerous systems (Freeman et al. 2013), as well as to other free and commercial PV modeling tools (Freeman et al. 2014).

SAM combines its PV plant performance model with an economic analysis that handles complex utility tariff structures, incentives, plant installation and operating cost information, and financial structures to calculate a full multi-year cash flow and some economic metrics, such as levelized cost of electricity (LCOE) and net present value (NPV). These outputs may facilitate decision making for people involved in the PV industry, such as project managers and engineers, policy analysts, technology developers, and energy systems researchers.

\footnotetext{
${ }^{11}$ See http://sam.nrel.gov.

12 See http://www.nrcan.gc.ca/energy/software-tools/7465.

${ }^{13}$ See http://freegreenius.dlr.de.
} 


\subsubsection{Yield Estimation of Concentrating Solar Technology Projects}

Concentrating solar technology (CST) plant yield models consist of two parts: One model for optical performance and another for the conversion of concentrated light to electricity, process heat, or chemical energy.

In what follows, a brief description of the available types of optical performance model is provided because that is where solar resource data are used as inputs. Some of these models include software modules for the ultimate conversion into electricity or for cost calculations (e.g., SAM, greenius, HFLCAL).

Optical performance models can be separated into different categories: Ray-tracing tools, analytical optical performance models, and models that determine the optical performance with lookup tables or parameterizations of the solar position relative to the collector.

\subsubsection{Ray-Tracing Tools}

The incident solar irradiance can be described as a multitude of solar rays transmitted from the sun to the concentrators and finally to the receiver. Ray-tracing tools such as STRAL (Belhomme et al. 2009), SolTRACE (Wendelin 2003), MIRVAL (Leary and Hankins 1979), or SPRAY (Buck 2010) calculate the path of the sun's rays from the sun's disk and its immediate sky annulus surrounding it (collectively referred to as "sunshape") to the receiver by application of physical laws. Monte Carlo techniques are often implemented to allow for tractable calculation times.

For the sake of illustration, one method of ray tracing that is available in SPRAY is explained here. The method selects one concentrator element after another and traces a given number of rays from the current element. After calculating the vector to the center of the sun, the appropriate sunshape is included. This is done by calculating an angular deviation of the ray vector from the center of the sun based on the probability density function corresponding to the user-defined sunshape. The specific ray under scrutiny is then related to a power calculated as the product of the incident DNI and the projected area of the current concentrator element divided by the number of rays per element. Then the path of the ray is followed until it reaches the receiver. This ray-tracing method can be based on actual measurements of the plant's geometry or on its design geometry varied by typical optical errors. In addition to the intercepted power, its distribution on the receiver is determined, so ray-tracing tools are also used for the detailed design of plant components.

\subsubsection{Analytical Optical Performance Models}

One example for a calculation method that uses an analytical approach is the Bendt-Rabl model (Bendt et al. 1979; Bendt and Rabl 1981). To accelerate calculations, analytical equations are derived and solved to describe the ray's path throughout the optical system. For example, the model can be used for parabolic troughs and solar dishes. In a first step, an angular acceptance function is determined from the design geometry. The angular acceptance function is defined by the fraction of rays incident on the aperture at a specific angle that ultimately reach the receiver. The second step consists of determining an effective source that includes both the user-defined sunshape and any possible deviation from the design geometry. The optical errors of a CST collector are described as Gaussian-distributed independent uncertainties. Their combination is also a Gaussian distribution with a standard deviation, which is often called an optical error. The function that describes the optical errors is then combined with the sunshape, using convolution. For line-focusing systems, such as parabolic troughs, a further integration step is required because the effect of circumsolar 
radiation on the incident irradiance depends strongly on angle. Finally, the intercepted radiation can be determined by summing the product of the effective source and the acceptance function over all angles.

Similar analytical methods are used in HELIOS (Vittitoe and Biggs 1981), DELSOL (Kistler 1986), and HFLCAL (Schwarzbözl et al. 2009).

\subsubsection{Lookup Table-Based Optical Performance Models}

The fastest way to determine the optical performance of a CST collector uses only parameterizations or lookup tables that describe the change of the optical performance with solar position. The necessary parameters can be derived from experimental data, the aforementioned analytical performance models, or ray-tracing tools. Such lookup tables or parameterizations are used in SAM (Blair et al. 2014) and greenius (Dersch et al. 2011; Quaschning et al. 2001). These examples also include the modeling of the conversion of the concentrated radiation to electricity or heat and economic analysis.

\subsubsection{Additional Meteorological Input Parameters for Yield Models}

GTI is by far the most important meteorological input parameter for PV models and DNI for CST models; however, further parameters must be provided for accurate yield analysis. The influence of ambient temperature and wind on the PV efficiency was briefly mentioned before. High wind speed might also force the plant operators to set the solar collectors or panels to their stow position. Thermal losses are influenced by wind (convection) and ambient temperature. Humidity and pressure have an effect on the thermodynamic performance mainly of a CSP plant. A discussion of the influence of the different meteorological parameters on CSP can be found in Chhatbar and Meyer (2011). Recently, other parameters such as soiling have gained interest in PV and CST projects. The soiling issue is investigated by Wolfertstetter et al. (2014) and Sarver et al. (2013). In the case of central receiver CSP plants, the extinction of the solar radiation between the heliostat and the receivers is another important meteorological input requested by developers. For extinction between heliostat and receiver, see the detailed review by Hanrieder et al. (2017).

\subsubsection{Additional Solar Radiation Characteristics for Yield Models}

Directly connected to the resource itself (GHI, GTI, or DNI) is its spectral and angular distribution. The case of the spectral distribution is mainly relevant to the case of PV projects because the energy yield of solar cells is a function of their spectral response and of the spectral distribution of the incident irradiance.

The angular distribution of the incident irradiance is not critical for PV systems, although smart-tracking systems can rapidly modify the tilt and/or azimuth of the GTI to maximize production in response to changing cloudiness. In the case of CST plants (mainly in the case of central receivers), the sunshape characterization is also of interest. Depending on the selected CST simulation software, the sunshape may have to be provided by the user. The first two types of models mentioned above - ray-tracing and analytical models - need both the sunshape and DNI as input variables. The third model type (lookup tables) requires only DNI as input because a default sunshape is fixed in the model. A discussion of the influence of the selection of the sunshape data can be found in Wilbert (2014). 


\subsection{Solar Resource Data for Power Plant Characterization and Operation}

This section discusses a variety of approaches for monitoring the solar resource at an existing power plant to better understand the plant's performance. The performance of a solar energy system is directly linked to the current meteorological conditions. For flat-plate thermal collectors and PV, the production is roughly proportional to the incident GTI. For CST, DNI is the most important parameter; however, additional meteorological variables need to be monitored, as discussed above. In summary, the real-time monitoring of meteorological conditions at the system's location is important to:

- Evaluate a performance guarantee (acceptance testing)

- Assess the power plant performance to improve yield predictions and gain knowledge toward improvements in future plants

- Identify conditions of poor performance, including evidence of soiling, shading, hardware malfunction, or degradation, which might lead to warranty replacement, etc.

\subsubsection{Performance Guarantee}

Different methods exist to evaluate the plant's performance guarantee. In all cases, on-site measurements of the solar resource are necessary.

For CST, acceptance tests involve DNI. For flat-plate thermal collectors and PV, the yield prediction is generally based on GHI (even though the actual resource corresponds to GTI). Hence, it is also common for a performance guarantee to use GHI as the basis for determining whether a plant has performed as promised; however, some companies have noted that the performance characterization of a PV plant can be accomplished with a lower uncertainty by using GTI instead (reducing the uncertainty inherent to the approximate transposition procedure transforms GHI into GTI). Moreover, specific irradiance sensors (such as reference cells or reference modules that closely match the PV module response) may be chosen to match the expected response of the PV modules (thus reducing angle-ofincidence and spectral effects). Specifying GHI remains the best option if, for instance, a PV system is constituted of different sections with POAs of different tilts or azimuths, which may be the case over complex terrain. If the performance guarantee is specified in terms of GTI, the plant efficiency characterized during the performance guarantee evaluation may differ from the efficiency that might have been estimated in an earlier step with a model rather than using historical GHI data. Also, the placement of all sensors must (1) be in the correct plane (which is easy to confirm when the sensor is in the horizontal plane but not as easy for other orientations); and (2) experience the expected local conditions (ground albedo and shading) if the sensor is not in the horizontal plane (Kurtz et al. 2014).

Additional meteorological variables must be measured, as discussed above for yield predictions. Depending on the size of the solar system, more than one measurement point must be considered if the evaluation takes place during partly cloudy-sky conditions.

Acceptance tests for CSP systems are discussed in Janotte et al. (2012) and Kearney (2013, 2009).

\subsubsection{Power Plant Performance Monitoring}

During power plant operation, knowledge of the current meteorological conditions and of the real-time status of the plant are of high importance. Also, the future meteorological

conditions are useful. Therefore, both solar resource measurements and forecasts are essential 
parts of many large solar systems. For CSP installations, such measurements and forecasts are fundamental. Although many PV plants can operate successfully with only episodic intervention, measurements and forecasts can also be advantageous. There can also be value to washing a PV array as a function of meteorological conditions (e.g., frequency of recent precipitation). Moreover, equipment malfunctions can be detected more quickly if the PV plant output is being continually compared to the expected output based on actual meteorological conditions. There is wide agreement that a matched reference cell in the POA is the best choice when the goal is to identify the need for power plant maintenance.

For CST, DNI measurements are involved along with the other parameters mentioned above.

\subsubsection{Solar Radiation Forecasting Needs for Solar Projects Operation and Maintenance}

Forecasting the production of a solar power plant can considerably improve its profitability (Ramirez and Vindel 2017). At the O\&M steps, accurate predictions of the plant-average solar resource are needed for both thermal and PV power plants. The most important forecasted parameter is GHI in the case of PV plants without concentration and DNI in the case of CSP plants.

Detailed explanations on solar radiation forecasting methodologies and the current state of the art are provided in Chapter 7. The specific forecasting needs depend on the intended application. Essentially, the solar radiation forecasts can be used for either planning maintenance works or for operation optimization.

\subsubsection{Planning Maintenance Works}

Maintenance work is needed in all types of solar plants. Examples of such work include technical closure, replacement of defective components, cleaning of modules or mirrors, or even conducting characterization tests. Depending on the expected duration of the maintenance work, the required forecasts correspond to different time horizons. Usually, a technical plant closure must be planned ahead of time and would occur at a fixed date, based on the long-term forecasting on a monthly basis, whereas minor maintenance work would be decided based on day-ahead forecasts.

\subsubsection{Operation and Revenue Optimization}

For operation optimization, forecasting knowledge will help the improvement of the electricity sales by better matching production with demand if the plant is equipped with storage, particularly in the case of CSP projects. In that case, the plant's annual revenue is conditioned by the quality of the solar forecasts (Ferretti et al. 2016). Thus, especially when subject to a fluctuating electricity market, the plant's revenue can be maximized if production can be predicted appropriately. If there are ramp rate limitations defined by the grid operator, the yield of a PV plant with batteries can be improved by storing excess PV energy during positive ramps and using energy from the batteries during negative ramps. Forecasts can help to manage the battery storage for this application and to limit the required storage capacity. In Figure 8-11, the role of meteorological variables in the demand and energy generation is shown. 


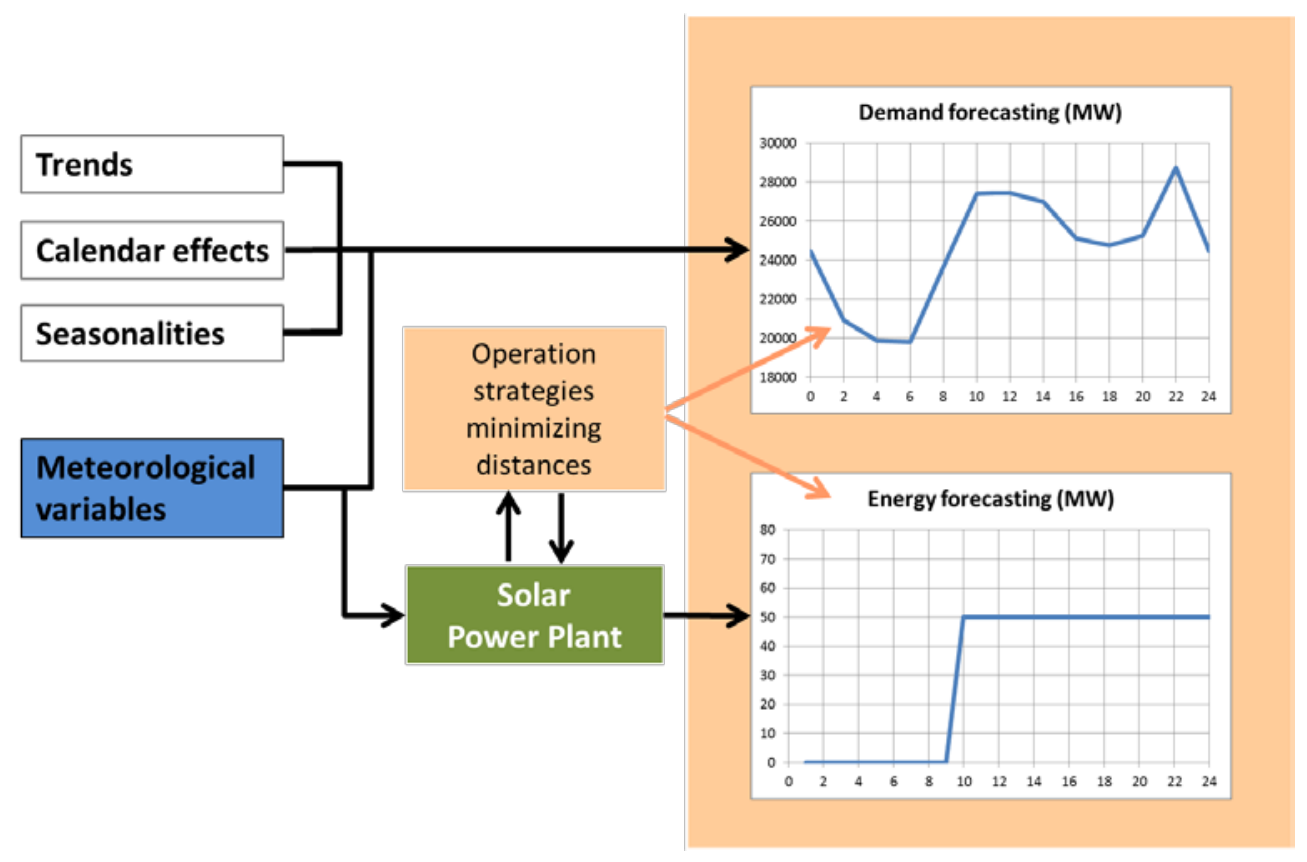

Figure 8-11. Importance of weather variables in forecasted demand and energy production. Image from Ramirez and Vindel 2017

\subsection{Applying Solar Resource Data to Other Types of Solar Energy Projects}

\subsubsection{Projects Using Flat-Plate Thermal Collectors}

Energy simulation tools for flat-plate thermal collector systems usually include a suite of modules describing the thermal receiver and thermal losses of the piping, parasitic losses, and thermal storage. Some traditional tools for these simulations are Polysun ${ }^{14}$ and $\mathrm{T}^{*}$ Sol. $^{15}$ However, some of the aforementioned software tools include these types of systems. This is the case for the software tools RETScreen, ${ }^{16} \mathrm{SAM},{ }^{17}$ or Greenius. ${ }^{18}$ Although the irradiance in the flat-plate collector plane (GTI) is the physically relevant irradiance, the separate specification of DNI and DHI can be of interest. Individual incidence angle modifiers can be used to determine the efficiency of the DNI and DHI conversion, respectively, for a given solar position.

\subsubsection{Solar Heating and Cooling in Buildings, Smart Cities, and Smart Grids}

Solar heating and cooling in buildings (SDHtake-off Project 2012), Smart Cities, and Smart Grids are projects that include solar systems among other energy systems or energy conservation measures. Solar radiation data are still needed for sizing, simulation, and evaluation. Note, in particular, that TMY and TRY/DRY (Crawley 1998; Hall et al. 1978; Lund 1974) were originally developed for building applications.

\footnotetext{
${ }^{14}$ See http://www.velasolaris.com/.

${ }^{15}$ See http:/www.valentin-software.com/.

${ }^{16}$ See http://www.nrcan.gc.ca/energy/software-tools/7465.

${ }^{17}$ See http://www.sam.nrel.gov.

${ }^{18}$ See http://freegreenius.dlr.de.
} 


\subsubsection{Chemical Applications}

Solar resource data are required for several chemical applications. These can be divided into two main topics due to their different use of the solar resource: desalination and photochemical applications.

\subsubsection{Solar Desalination}

Global demand for freshwater is continuously increasing due to population growth and economic development. To meet this increasing demand, desalination has become the most important source of freshwater for drinking and agriculture in some world regions with huge solar energy potentials, such as the Middle East and North Africa region (MENA) (Isaka 2012).

The main desalination technologies include (Goswami 2015):

- Thermal processes using either thermal energy and/or electricity as the energy input. Thermal desalination involves distillation processes where saline water is heated to vaporize it in order to subsequently obtain freshwater from the steam cooling and condensation.

- Membrane-based processes using only electricity as energy input. This kind of desalination uses reverse osmosis (RO) membranes to separate freshwater from saline water; saline feed-water is brought to the surface of a membrane, which selectively lets water traverse it while blocking salts.

Solar thermal desalination consists of desalinating seawater via Multi Stage Flash (MSF) or Multi Effect Distillation (MED), using solar heat as the energy input. This methodology is the most promising desalination process based on renewable energy. A desalination plant consists of two parts: a solar heat collector and a distiller. Desalination plants associated with an STE power plant are considered very promising. In this case it is possible to use both the thermal energy and electricity produced from the plant, allowing a variety of design solutions that combine electricity power and heat generation with water desalination. As previously discussed, the STE part of the desalination project needs several years of high-quality on-site data for simulation and design optimization purposes or site-adapted data time series of low bias, similar to regular STE plants.

A system using PV technology can alternatively be connected directly to membrane desalination processes, such as RO or electrodialysis (ED), which use electricity as energy input. Many small PV-based desalination systems have been installed worldwide, especially in remote areas and islands. As in the case of a standard PV plant, GTI data are needed as the most relevant solar input for these systems.

\subsubsection{Solar Photocatalysis: Detoxification and Disinfection of Fluids}

Solar photocatalytic detoxification and disinfection processes constitute a solution for the treatment of contaminated groundwater, industrial wastewater, air, or soil (Malato 2004). The development of these processes has reached a point where the solar technology can be competitive with conventional treatment methods, particularly in isolated locations with high solar potential, which can be the case at many agricultural farms.

Solar photochemistry can be defined as the technology that collects solar photons and introduces them in an adequate reactor volume to promote specific chemical reactions (Blanco and Malato 2010). The equipment that performs this function is a solar collector. 
Traditionally, solar collector systems have been classified into three types depending on the level of concentration attained by them, which is directly related to the achievable system temperature. For photochemical applications more specifically, compound parabolic collector (CPC) photo-reactors are used. CPCs are static concentrators with a relatively large acceptance angle. Hence, they can utilize DNI and the part of DHI that emanates from the circumsolar region. Their low concentration ratio is enough to make the thermal fluid inside them reach temperatures over $100^{\circ} \mathrm{C}$. Figure 8-12 illustrates two different photo-reactors installed at Plataforma Solar de Almería (PSA) ${ }^{19}$ (CIEMAT, Spain).
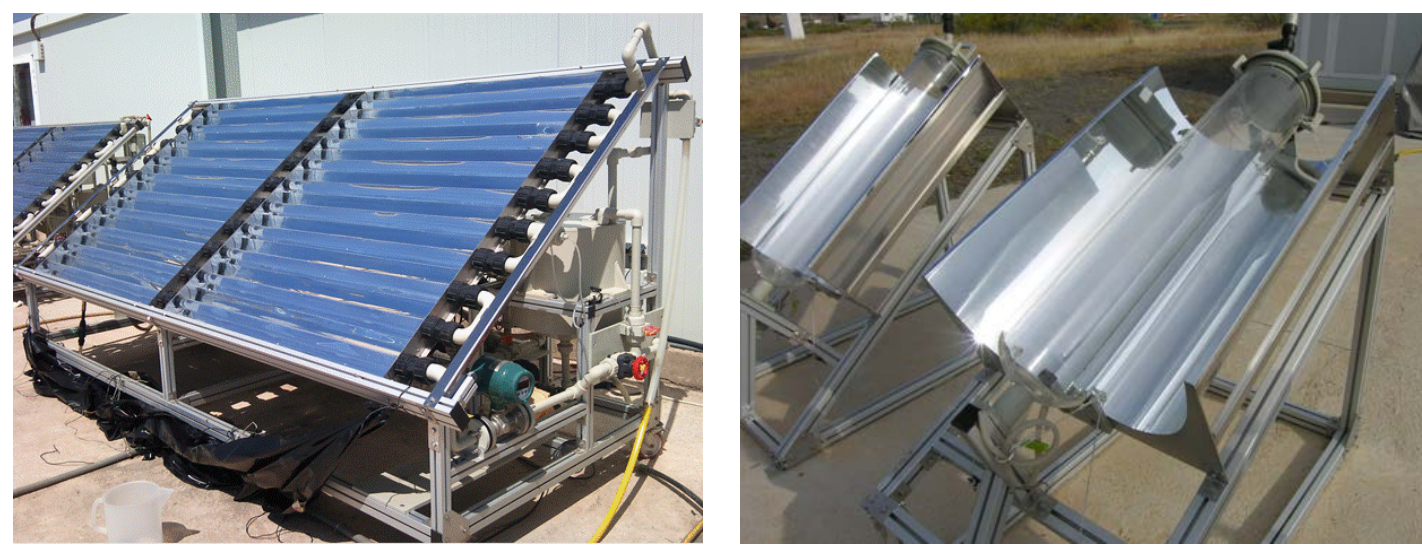

Figure 8-12. CPC photo-reactors installed at PSA for solar water disinfection applications: (left) CPC-SODIS, (right) FITOSOL-2. (Source: PSA, CIEMAT http://www.psa.es/en/instalaciones/aguas.php)

The photochemical reactor must contain the working fluid, including the catalyst or sensitizer, and must transmit solar UV light efficiently with minimal pressure drop across the system. Also, it must provide good mass transfer from the fluid stream to an illuminated photocatalyst or sensitizer surface in order to have a reaction rate as high as possible.

The requirements for solar photochemical reactors are similar to any other photochemical reactor, with the particularity that their light input comes from the sun rather than from a lamp. For this reason, and according to the working temperature, the collector must be tilted or mounted on a tracking system with one or two axes. Depending on the type of solar collector, tilted or direct UV solar irradiance data will be needed. In most cases, this variable must be modeled from DNI and/or GHI data.

\subsection{Summary of Application of Solar Resource Data}

This chapter summarized available information as well as guidance on the type of solar resource relevant to each stage of a solar project. In a general introduction, the chapter goals were described as well as several questions related to the topics it covers. This chapter attempted to inform readers about their specific needs relative to solar radiation data and how this depends on the type of solar project and on the project's stage. Figure 8-3 showed a summary of the solar radiation needs at different steps of the project. The information provided here is applicable to the case of big solar energy projects, mainly PV and STE, and also to the case of building energy performance evaluations.

${ }^{19}$ See http://www.psa.es/en/instalaciones/aguas.php. 
Maps should be used to make a preliminary assessment of the solar resource, cautiously assuming a fairly large potential for error. GIS tools and resources are commonly used at this step for convenience. Various spatial resolutions need to be used when addressing projects at the regional or national scale - compared to the case of the neighborhood or city scale. Using these tools, maps of solar radiation, and simple energy production models, the potential of the energy output from different technologies can be evaluated and compared. Using GIS tools for potential assessment, terrain slopes and additional land constraints have to be considered. During this screening process, the nature of local aerosols and their spatio-temporal variability may have to be considered. Because widely different sources of information may be available at that stage, it is important to define appropriate comparison parameters among the solar resource data sources and clarify the definition of variability, error, and uncertainty. Thus, variability has to be identified mainly at the inter-annual level and distinguished from the uncertainty of the model. Ideally, taking into account the uncertainties from each data source, a common "most probable" range should be obtained, which should include the expected or "true" value. Temporal and spatial variability is addressed in this chapter through the COV, which can be determined by using long time series of measured or modeled data for the site and its surroundings.

In the feasibility assessment stage, typical solar radiation series are needed for plant simulation and economic analysis. Typical annual time series are provided by a TMY, TRY, DRY, TGY, or TDY. In addition to the review of typical meteorological data series generation for solar energy simulation, this chapter reviewed the proposed procedures for the analysis of the inter-annual variability and the generation of series of a specific "Probabilities of Exceedance," usually the POE90. Thus, in order to evaluate the profitability and payback of a project, simulations of its behavior during bad years are needed. A specific section dealt with combining data sets and site adaptation processes. These steps are very important for a precise feasibility assessment and to guarantee bankability, particularly for projects with large associated investments.

During or before the plant's construction phase, solar radiation data are needed to refine the yield estimation and minimize the expected profitability uncertainties. Thus, the value of the energy generated by a solar installation depends on the system's output and on the price offered for that energy at the time it is generated. Methods for yield estimation of nonconcentrating PV projects and of CST projects have been discussed. Additional meteorological inputs that are necessary for yield estimation, as well as solar radiation characteristics such as its spectral and angular distribution, were also discussed.

For power plant characterization and operation, the solar resource information must include high-quality on-site measurements (to qualify the plant's operation) as well as accurate irradiance forecasts (for operation and revenue optimization).

This chapter's final section discussed the type of solar radiation data needed for different types of solar projects (flat-plate thermal collectors, solar heating and cooling in buildings, smart cities and smart grids, solar desalination, and other chemical applications). 


\section{References}

AENOR, 2014. Centrales termosolares. Procedimiento de generación de Año Solar Representativo, UNE. UNE 206011.

Andersen, B., Eidorff, S., Lund, H., Pedersen, E., Rosenørn, S., Valbjørn, O., 1974.

Referenceåret - Vejrdata for VVS beregninger, (The Reference Year - Weather data for HVAC-calculations), Report no. 89. Danish Building Research Institute.

Belhomme, B., Pitz-Paal, R., Schwarzbozl, P., Ulmer, S., 2009. A New Fast Ray Tracing Tool for High-Precision Simulation of Heliostat Fields. Journal of Solar Energy Engineering. 131, 31002. doi:10.1115/1.3139139

Bendt, P., Rabl, A., 1981. Optical analysis of point focus parabolic radiation concentrators. Applied Optics. 20, 674. doi:10.1364/AO.20.000674

Bendt, P., Rabl, A., Gaul, H.W., Reed, K.A., 1979. Optical analysis and optimization of line focus solar collectors, SERI/TR-34-09. Solar Energy Research Institute, Golden, Colorado.

Benseman, R.F., Cook, F.W., 1969. Solar radiation in New Zealand--The standard year. New Zeal. J. Sci. 12, 698-708.

Bernardos, A., Ramírez, L., Martín, L., Gastón, M., Vindel, J.M., Liria, J., FernandezPeruchena, C.M., 2015. Solar Resource Mapping in Tanzania, The World Bank Documents \& Reports.

Beyer, H.G., Dumortier, D., Wald, L., Martinoli, M., Schilings, C., Gschwind, B., Menard, L., Gaboardi, E., Ramirez-Santigosa, L., Polo, J., Cebecauer, T., Huld, T., Suri, M., de Blas, M., Lorenz, E., Pfatischer, R., Remund, J., Ineichen, P., Tsvetkov, A., Hofierka, J., 2008. Management and Exploitation of Solar Resource Knowledge, in: EUROSUN Conference. Lisbon, Portugal.

Blair, N., Dobos, A.P., Freeman, J., Neises, T., Wagner, M., Ferguson, T., Gilman, P., Janzou, S., 2014. System advisor model, SAM 2014.1. 14: General description, NREL/TP6A20-61019. National Renewable Energy Laboratory, Golden, Colorado.

Blanc, P., Boilley, A., Killius, N., Lefevre, M., Massip, P., Schroedter-Homscheidt, M., Wald, L., 2012. Methods for Data Fusion, in: Project Endorse.

Blanco, J., Malato, S., 2010. Solar Energy Conversion and Photoenergy System - Thermal Systems and Desalination Plants: v.3., EOLSS. EOLSS Publishers Co Ltd.

Buck, R. 2010. Solar Power Raytracing Tool, SPRAY, User Manual. Version 2.6, 20.3.2010. DLR.

Cebecauer, T., Suri, M., 2015. Typical Meteorological Year Data: SolarGIS Approach. Energy Procedia 69, 1958-1969. doi:10.1016/j.egypro.2015.03.195

Cebecauer, T., Šúri, M., Gueymard, C.A., 2011. Uncertainty sources in satellite-derived direct normal irradiance: How can prediction accuracy be improved globally?, SolarPACES Conference. Granada, Spain. 
Chhatbar, K., Meyer, R., 2011. The Influence of Meteorological Parameters on the Energy Yield of Solar Thermal Power Plants, SolarPACES Conference. Granada, Spain.

Crawley, D.B., 1998. Which Weather Data Should You Use for Energy Simulations of Commercial Buildings?, in: ASHRAE Transactions. 104 (2), TO-98-2-2, p. 18.

Crowley, T.J., Unterman, M.B., 2013. Technical details concerning development of a $1200 \mathrm{yr}$ proxy index for global volcanism. Earth System Science Data, 5:187-197. doi:10.5194/essd5-187-2013

Dersch, J., Schwarzbözl, P., Richert, T., 2011. Annual Yield Analysis of Solar Tower Power Plants With GREENIUS. Journal of Solar Energy Engineering. 133, 31017.

doi:10.1115/1.4004355

Dobos, A., Gilman, P., Kasberg, M., 2012. P50/P90 Analysis for Solar Energy Systems Using the System Advisor Model Preprint, in: World Renewable Energy Congress.

NREL/CP-6A20-54488, Denver, Colorado.

Dobos, A.P., 2014. PVWatts Version 5 Manual (NREL/TP-6A20-62641).

Ellis, A., Behnke, M., Barker, C., 2011. PV System models for Grid Planning Studies, in: 37th IEEE Photovoltaic Specialists Conference Proceedings. Seattle, p. 5.

Espinar, B., Ramírez, L., Drews, A., Beyer, H.G., Zarzalejo, L.F., Polo, J., Martín, L., 2009. Analysis of different comparison parameters applied to solar radiation data from satellite and German radiometric stations. Solar Energy, 83:118-125. doi:10.1016/j.solener.2008.07.009

Fernández-Peruchena, C.M., Gastón, M., Sánchez, M., García-Barberena, J., Blanco, M., Bernardos, A., 2015. MUS: A multiscale stochastic model for generating plausible meteorological years designed for multiyear solar energy yield simulations. Solar Energy, 120: 244-256. doi:10.1016/j.solener.2015.07.037

Fernández Peruchena, C.M., Ramírez, L., Silva-Pérez, M.A., Lara, V., Bermejo, D., Gastón, M., Moreno-Tejera, S., Pulgar, J., Liria, J., Macías, S., Gonzalez, R., Bernardos, A., Castillo, N., Bolinaga, B., Valenzuela, R.X., Zarzalejo, L.F., Ramı, L., Silva-pe, M.A., Ferna, C.M., Moreno-Tejera, S., Lara, V., Bermejo, D., Liria, J., Macı, S., Bolinaga, B., Valenzuela, R.X., Zarzalejo, L.F., 2016. A statistical characterization of the long-term solar resource: Towards risk assessment for solar power projects. Solar Energy, 123: 29-39. doi:

http://dx.doi.org/10.1016/j.solener.2015.10.051

Ferretti, F., Hogendijk, C., Aga, V., Ehrsam, A., 2016. Addressing Forecast Uncertainty Impact on CSP Annual Performance, in: SolarPACES Conference. Abu Dhabi, UAE.

Freeman, J., Whitmore, J., Blair, N., Dobos, A.P., 2014. Validation of multiple tools for flat plate photovoltaic modeling against measured data, NREL/TP-6A20-61497. National Renewable Energy Laboratory, Golden, Colorado.

Freeman, J., Whitmore, J., Kaffine, L., Blair, N., Dobos, A.P., 2013. System Advisor Model: Flat Plate Photovoltaic Performance Modeling Validation Report, NREL/TP-6A20-60204.

National Renewable Energy Laboratory, Golden, Colorado. 
Goswami, D.Y., 2015. Principles of Solar Engineering, Third Edition, CRC Press. Boca Raton. doi:10.1088/1751-8113/44/8/085201

Grantham, A.P., Pudney, P.J., Ward, L.A., Belusko, M., Boland, J.W., 2017. Generating synthetic five-minute solar irradiance values from hourly observations. Solar Energy, 147: 209-221. doi:10.1016/j.solener.2017.03.026

Gueymard, C.A., 2014. A review of validation methodologies and statistical performance indicators for modeled solar radiation data: Towards a better bankability of solar projects. Renewable and Sustainable Energy Reviews, 39:1024-1034. doi:10.1016/j.rser.2014.07.117

Gueymard, C.A., 2012. Temporal variability in direct and global irradiance at various time scales as affected by aerosols. Solar Energy, 86:3544-3553.

doi:10.1016/j.solener.2012.01.013

Gueymard, C.A., George, R., 2005. Gridded aerosol optical depth climatological datasets over continents for solar radiation modeling, in: ISES Solar World Congress. Orlando, Florida.

Gueymard, C.A., Gustafson, W.T., Bender, G., Etringer, A., Storck, P., 2012. Evaluation of procedures to improve solar resource assessments: Optimum use of short-term data from a local weather station to correct bias in long-term satellite-derived solar radiation time series, in: World Renewable Energy Forum, Denver, CO, American Solar Energy Society.

Gueymard, C.A., Wilcox, S.M., 2011. Assessment of spatial and temporal variability in the US solar resource from radiometric measurements and predictions from models using ground-based or satellite data. Solar Energy, 85:1068-1084.

doi:10.1016/j.solener.2011.02.030

Gueymard, C., Wilcox, S., 2009. Spatial and temporal variability in the solar resource: Assessing the value of short-term measurements at potential solar power plant sites, in: ASES National Solar Conference. Buffalo, New York.

Habte, A., Lopez, A., Sengupta, M., Wilcox, S., 2014. Temporal and Spatial Comparison of Gridded TMY, TDY, and TGY Data Sets, NREL/TP-5D00-60886. National Renewable Energy Laboratory, Golden, Colorado.

Hall, I.J.R., Prairie, R.R., Anderson, H.E., Boes, E.C., 1978. Generation of Typical Meteorological Years for 26 SOLMET Stations, SAND78-1601. Sandia National Laboratories, Albuquerque, NM, USA.

Hanrieder, N., Wilbert, S., Mancera-Guevara, D., Buck, R., Giuliano, S., Pitz-Paal, R., 2017. Atmospheric extinction in solar tower plants - A review. Solar Energy, 152: 193-207. doi:10.1016/j.solener.2017.01.013

Hirsch, T., Schenk, H., Schmidt, N., Meyer, R., 2010. Dynamics of oil-based Parabolic Trough Plants - impact of transient behaviour on energy yield, in: SolarPACES Conference. Perpignan, France.

Ho, C.K., Khalsa, S.S., Kolb, G.J., 2011. Methods for probabilistic modeling of concentrating solar power plants. Solar Energy, 85:669-675. doi:10.1016/j.solener.2010.05.004 
Ho, C.K., Kolb, G.J., 2010. Incorporating Uncertainty into Probabilistic Performance Models of Concentrating Solar Power Plants. Journal of Solar Energy Engineering, 132:31012. doi:10.1115/1.4001468

Hofmann, M., Riechelmann, S., Crisosto, C., Mubarak, R., Seckmeyer, G., 2014. Improved Synthesis of Global Irradiance with One-Minute Resolution for PV System Simulations. International Journal of Photoenergy, 1-10. doi:10.1155/2014/808509

Huang, Y., Chen, Z., Wu, B., Chen, L., Mao, W., Zhao, F., Wu, J., Wu, J., Yu, B., 2015. Estimating Roof Solar Energy Potential in the Downtown Area Using a GPU-Accelerated Solar Radiation Model and Airborne LiDAR Data. Remote Sensing, 7:17212-17233. doi: $10.3390 /$ rs 71215877

Ineichen, P., Perez, R., 2002. A new airmass independent formulation for the linke turbidity coefficient. Solar Energy, 73:151-157. doi:10.1016/S0038-092X(02)00045-2

International Electrotechnical Commission, 1998. Photovoltaic system performance monitoring - Guidelines for measurement, data exchange and analysis, IEC 61724.

IPCC, 2000. Summary for Policymakers: Emissions Scenarios. A Special Report of Working Group III of the Intergovernmental Panel on Climate Change, Group. doi:92-9169-113-5

Isaka, M., 2012. Water desalination using renewable energy. Technology brief. IEA-ETSAP \& IRENA.

ISO 15927-4, 2005. Hygrothermal performance of buildings -- Calculation and presentation of climatic data -- Part 4: Hourly data for assessing the annual energy use for heating and cooling. International Organization for Standardization, Geneva, Switzerland.

Jakubiec, J.A., Reinhart, C.F., 2013. A method for predicting city-wide electricity gains from photovoltaic panels based on LiDAR and GIS data combined with hourly Daysim simulations. Solar Energy, 93:127-143. doi:10.1016/j.solener.2013.03.022

Janotte, N., Lüpfert, E., Pitz-Paal, R., 2012. Acceptance Testing and Advanced Evaluation Strategies for Commercial Parabolic Trough Solar Fields, SolarPACES Conference. Marrakesch, Morocco.

Janotte, N., Wilbert, S., Sallaberry, F., Schroedter-Homscheidt, M., Ramirez, L., 2017. 2 Principles of CSP performance assessment, The Performance of Concentrated Solar Power (CSP) Systems. pp. 31-64. doi:10.1016/B978-0-08-100447-0.00002-X

Kearney, D., 2013. Utility-Scale Power Tower Solar Systems: Performance Acceptance Test Guidelines.

Kearney, D., 2009. Utility-Scale Parabolic Trough Solar Systems: Performance Acceptance Test Guidelines: April 2009 - December 2010, NREL/SR-5500-48895. National Renewable Energy Laboratory, Golden, Colorado.

Kistler, B.L., 1986. A User's Manual for DELSOL3: A Computer Code for Calculating the Optical Performance and Optimal System Design for Solar Thermal Central Receiver Plants, SAND86-8018. 
Kurtz, S., Krishnani, P., Freeman, J., Flottemesch, R., Riley, E., Dierauf, T., Newmiller, J., Ngan, L., Jordan, D., Kimber, A., 2014. PV system energy test, in: 2014 IEEE 40th Photovoltaic Specialist Conference (PVSC). IEEE, pp. 0879-0884.

doi:10.1109/PVSC.2014.6925055

Lara-Fanego, V., Pulgar, J., Fernández-Peruchena, C.M., Gastón, M., Moreno, S., Ramírez, L., Valenzuela, R.X., Bermejo, D., Silva, M., Pavón, M., Bernardos, A., Zarzalejo, L.F., Macías, S., 2016. A novel procedure for generating solar irradiance TSYs, SolarPACES Conference. Abu Dhabi, UAE.

Le, T., Kholdi, D., Xie, H., Dong, B., Vega, R., 2016. LiDAR-Based Solar Mapping for Distributed Solar Plant Design and Grid Integration in San Antonio, Texas. Remote Sens. 8, 247. doi: $10.3390 /$ rs 8030247

Leary, P.L., Hankins, J.D., 1979. User's guide for MIRVAL: a computer code for comparing designs of heliostat-receiver optics for central receiver solar power plants, SAND-77-8280. Sandia National Laboratories, Livermore, CA (USA).

Lund, H., 1995. Design Reference Years. Task 9, Solar Heating and Cooling. International Energy Agency.

Lund, H., 1974. The "Reference Year", a set of climatic data for Environmental Engineering. Also published as: Report no. 32, Thermal Insulation Laboratory, Technical University of Denmark, in: Second Symposium on the Use of Computers for Environmental Engineering Related to Building. Paris (France), p. 12.

Malato, S., 2004. Photocatalitic reactors for the treatment of liquid wastewater in the presence of solar radiation, in: 1st Seminar of "Advanced Oxidation Methods of the Treatment of Liquid and Air Waste." Thessaloniki , p. 16.

Marion, W., Urban, K., 1995. User's Manual for TMY2's, Typical Meteorological Years, Derived from the 1961-1990 National Solar Radiation Data Base, NREL. National Renewable Energy Laboratory, Golden, Colorado.

Martínez-Rubio, A., Sanz-Adan, F., Santamaría-Peña, J., Martínez, A., 2016. Evaluating solar irradiance over facades in high building cities, based on LiDAR technology. Applied Energy, 183:133-147. doi:10.1016/j.apenergy.2016.08.163

Mehos, M., Perez, R., 2005. Mining for Solar Resources: U.S. Southwest Provides Vast Potential, NREL/JA-550-37799. National Renewable Energy Laboratory, Golden, Colorado.

Meybodi, M.A., Ramírez-Santigosa, L., Beath, A.C., 2017. A Study on the Impact of Time Resolution in Solar Data on the Performance CSP Plants. Renewable Energy. doi: http://dx.doi.org/10.1016/j.renene.2017.03.024

Meyer, R., Geuder, N., Lorenz, E., Hammer, A., Ossietzky, C. Von, Oldenburg, U., Beyer, H.G., 2008. Combining solar irradiance measurements and various satellite-derived products to a site-specific best estimate, in: SolarPACES Conference. pp. 1-8.

Mieslinger, T., Ament, F., Chhatbar, K., Meyer, R., 2014. A New Method for Fusion of Measured and Model-derived Solar Radiation Time-series. Energy Procedia 48, 1617-1626. doi:10.1016/j.egypro.2014.02.182 
Mohajeri, N., Upadhyay, G., Gudmundsson, A., Assouline, D., Kämpf, J., Scartezzini, J.-L., 2016. Effects of urban compactness on solar energy potential. Renewable Energy, 93:469482. doi:10.1016/j.renene.2016.02.053

Moody's Incestors Services, July 28, 2010. PV Solar Power Generation Projects. Special Comment.

Navarro, A.A., Ramírez, L., Domínguez, P., Blanco, M., Polo, J., Zarza, E., 2016. Review and validation of Solar Thermal Electricity potential methodologies. Energy Conversion Management, 126: 42-50. doi:10.1016/j.enconman.2016.07.070

Nielsen, K.P., Blanc, P., Vignola, F., Ramírez, L., Blanco, M., Meyer, R., 2017. Discussion of currently used practices for: "Creation of Meteorological Data Sets for CSP/STE Performance Simulations." SolarPACES rep., http://www.solarpaces.org/images/BeyondTMY_Discussion_of_current_methods_v3 0.pdf.

Pavón, M., Fernández, C.M., Silva, M., Moreno, S., Guisado, M. V., Bernardos, A., 2016. Statistical Analysis of CSP Plants by Simulating Extensive Meteorological Series, in: SolarPACES Conference. Abu Dhabi, UAE.

Peruchena, C.M.F., Ramírez, L., Silva, M., Lara, V., Bermejo, D., Gastón, M., Moreno, S., Pulgar, J., Liria, J., Macías, S., Gonzalez, R., Bernardos, A., Castillo, N., Bolinaga, B., Valenzuela, R.X., Zarzalejo, L., 2016. A methodology for calculating percentile values of annual direct normal solar irradiation series, in: SolarPACES Conference. p. 150005. doi:10.1063/1.4949237

Polo, J., Wilbert, S., Ruiz-Arias, J.A., Meyer, R., Gueymard, C., Súri, M., Martín, L., Mieslinger, T., Blanc, P., Grant, I., Boland, J., Ineichen, P., Remund, J., Escobar, R., Troccoli, A., Sengupta, M., Nielsen, K.P., Renne, D., Geuder, N., Cebecauer, T., 2016. Preliminary survey on site-adaptation techniques for satellite-derived and reanalysis solar radiation datasets. Solar Energy, 132:25-37. doi:10.1016/j.solener.2016.03.001

Quaschning, V., Ortmanns, W., Kistner, R., Geyer, M., 2001. Greenius: A New Simulation Environment for Technical and Economical Analysis of Renewable Independent Power Projects, Solar Forum 2001. pp. 413-418.

Ramírez, L., Barnechea, B., Bernardos, A., Bolinaga, B., Cony, M., Moreno, S., Orive, R., Polo, J., Redondo, C., Salbidegoitia, I.B., Serrano, L., Tovar, J., Zarzalejo, L.F., 2012. Towards the standardization of procedures for solar radiation data series generation, SolarPACES Conference. p. 5.

Ramírez, L., Pagh, K., Vignola, F., Blanco, M., Blanc, P., Meyer, R., Wilbert, 2017. Road Map for Creation of Advanced Meteorological Data Sets for CSP Performance Simulations, SolarPACES rep. http://www.solarpaces.org/images/SolarPACES-

T5 BeyondTMY_Road_Map.pdf.

Ramirez, L., Vindel, J.M., 2017. Forecasting and nowcasting of DNI for concentrating solar thermal systems, in: Blanco, M., Ramirez Santigosa, L. (Eds.), Advances in Concentrating Solar Thermal Research and Technology. Elsevier, pp. 293-310. doi:10.1016/B978-0-08100516-3.00013-7 
Renné, D.S., 2017. Effective Solar Resource Methodologies for Sustainable PV Applications, in: Photovoltaics for Sustainable Electricity and Buildings., A. Sayigh, Ed. Springer International Publishing, pp. 25-53. doi:10.1007/978-3-319-39280-6_3

Renné, D.S., 2016. Resource assessment and site selection for solar heating and cooling systems, in: Advances in Solar Heating and Cooling, R. Wang and T. Ge, Eds. Elsevier, pp. 13-41. doi:10.1016/B978-0-08-100301-5.00002-3

Robock, A., 2000. Volcanic eruptions and climate. Review in Geophysics, 38:191-219. doi:10.1029/1998RG000054

Ruiz-Arias, J.A., Quesada-Ruiz, S., Fernández, E.F., Gueymard, C.A. 2015. Optimal combination of gridded and ground-observed solar radiation data for regional solar resource assessment. Solar Energy, 112:411-424. doi:10.1016/j.solener.2014.12.011.

Ruiz-Arias, J.A., Gueymard, C.A., Quesada-Ruiz, S., Santos-Alamillos, F.J., Pozo-Vázquez, D., 2016. Bias induced by the AOD representation time scale in long-term solar radiation calculations. Part 1: Sensitivity of the AOD distribution to the representation time scale. Solar Energy, 137:608-620. doi:10.1016/j.solener.2016.06.026.

Santos, T., Gomes, N., Freire, S., Brito, M.C., Santos, L., Tenedório, J.A., 2014. Applications of solar mapping in the urban environment. Applied Geography, 51:48-57.

doi:10.1016/j.apgeog.2014.03.008

Sarver, T., Al-Qaraghuli, A., Kazmerski, L.L., 2013. A comprehensive review of the impact of dust on the use of solar energy: History, investigations, results, literature, and mitigation approaches. Renewable and Sustainable Energy Reviews, 22:698-733.

doi:10.1016/j.rser.2012.12.065

Schumann, K., Beyer, H.G., Chhatbar, K., Meyer, R., 2011. Improving Satellite-Derived Solar Resource Analysis with Parallel Ground-Based Measurements. ISES Solar World Congress.

Schwarzbözl, P., Pitz-Paal, R., Schmitz, M., 2009. Visual HFLCAL - A Software Tool for Layout and Optimisation of Heliostat Fields, SolarPACES Conference. Berlin, Germany.

SDHtake-off Project, 2012. Solar district heating guidelines. Collection of fact sheets WP3 D3.1 \&amp; D3.2. Intelligent Energy. EU.

Sengupta, M., Habte, A., Kurtz, S., Dobos, A., Wilbert, S., Lorenz, E., Stoffel, T., Renné, D., Gueymard, C., Myers, D., Wilcox, S., Blanc, P., Perez, R., 2015. Best practices handbook for the collection and use of solar resource data for solar energy applications, NREL/TP-5D0063112. National Renewable Energy Laboratory, Golden, Colorado.

http://www.nrel.gov/docs/fy15osti/63112.pdf

Sigl, M., Winstrup, M., McConnell, J.R., Welten, K.C., Plunkett, G., Ludlow, F., Büntgen, U., Caffee, M., Chellman, N., Dahl-Jensen, D., Fischer, H., Kipfstuhl, S., Kostick, C., Maselli, O.J., Mekhaldi, F., Mulvaney, R., Muscheler, R., Pasteris, D.R., Pilcher, J.R., Salzer, M., Schüpbach, S., Steffensen, J.P., Vinther, B.M., Woodruff, T.E., 2015. Timing and climate forcing of volcanic eruptions for the past 2,500 years. Nature 523, 543-9.

doi:10.1038/nature14565 
Skartveit, A., Lund, H., Olseth, J.A., 1994. The Design Reference Year, DNMI Det Norske Meteorlogiske institutt.

Tomson, T., Russak, V., Kallis, A., 2008. Dynamic Behavior of Solar Radiation, in: Modeling Solar Radiation at the Earth's Surface. Springer Berlin Heidelberg, Berlin, Heidelberg, pp. 257-281. doi:10.1007/978-3-540-77455-6_10

Tooke, T.R., Coops, N.C., Christen, A., Gurtuna, O., Prévot, A., 2012. Integrated irradiance modelling in the urban environment based on remotely sensed data. Solar Energy, 86:29232934. doi:10.1016/j.solener.2012.06.026

van Sark, W.G.J.H.M., N.H., R., Müller, B., 2012. Review of PV Performance Ratio Development, World Renewable Energy Congress. Denver, Colorado, pp. 1-6.

doi:10.13140/2.1.2138.7204

Vignola, F., Grover, C., Lemon, N., McMahan, A., 2012. Building a bankable solar radiation dataset. Solar Energy, 86:2218-2229. doi:10.1016/j.solener.2012.05.013

Vignola, F.E., McMahan, A.C., Grover, C.N., 2013. Bankable Solar-Radiation Datasets, Solar Energy Forecasting and Resource Assessment. Elsevier, pp. 97-131. doi:10.1016/B978-0-12-397177-7.00005-X

Vittitoe, C.N., Biggs, F., 1981. User's guide to HELIOS: A computer program for modeling the optical behavior of reflecting solar concentrators. Part 1: Introduction and code input, NASA STI/Recon Technical Report N. NASA Astrophysics Data System.

Wendelin, T., 2003. SolTRACE: A New Optical Modeling Tool for Concentrating Solar Optics, in: Solar Energy. ASME, pp. 253-260. doi:10.1115/ISEC2003-44090

Wilbert, S., 2014. Determination of Circumsolar Radiation and its Effect on Concentrating Solar Power, PhD Thesis (RWTH-CONV-145307). Faculty of Mechanical Engineering. RWTH Aachen University.

Wilcox, S., 2007. National Solar Radiation Database 1991-2005 Update: User's Manual, NREL/TP-581-41364. National Renewable Energy Laboratory, Golden, Colorado. doi:10.2172/901864

Wilcox, S., Marion, W., 2008. Users Manual for TMY3 Data Sets, NREL/TP-581-43156. National Renewable Energy Laboratory, Golden, Colorado.

Wolfertstetter, F., Pottler, K., Geuder, N., Affolter, R., Merrouni, A.A., Mezrhab, A., PitzPaal, R., 2014. Monitoring of Mirror and Sensor Soiling with TraCS for Improved Quality of Ground based Irradiance Measurements. Energy Procedia, 49:2422-2432. doi:10.1016/j.egypro.2014.03.257 


\title{
9 Future Work
}

\author{
Jan Remund, ${ }^{1}$ Jan Kleissl, ${ }^{2}$ and Stefan Wilbert ${ }^{3}$ \\ 1. Meteotest \\ 2. University of California \\ 3. German Aerospace Center (DLR)
}

Advancing renewable energy technologies will require improvements to our understanding of solar radiation resources. This chapter briefly describes the areas of research and development identified as emerging technology needs. The International Energy Agency (IEA) Photovoltaic Power System (PVPS) Task 16 work plan on "solar resource for high penetration and large-scale applications" seeks to address significant parts of the research and development needs presented below.

\subsection{Forecasting Solar Radiation and Solar Power}

Solar power forecasting will be an essential component of the future energy supply system using large amounts of variable solar power. Currently, solar power forecasting systems already contribute to the successful integration of considerable amounts of solar power to the power grid.

Current research in irradiance and solar power forecasting covers all the different approaches presented in the earlier chapters of this handbook. Improvements in Numerical Weather Prediction (NWP)-based irradiance forecasting may emerge from improvements in resolution, data assimilation, and parameterizations of clouds and radiation. In particular, the development and application of rapid-update-cycle models ${ }^{1}$ has a high potential to improve intraday forecasting. However, such models need to be properly initialized. Hence, satellite detection of cloud height and optical depth as well as other atmospheric states remain research priorities. A further opportunity emerges from the expansion of Large Eddy Simulation (LES) approaches to larger and larger domain sizes that may soon bring operational mesoscale forecasting within reach (Schalkwijk et al. 2015). LES will reduce the need for cloud parameterization approaches that are challenged by the disparity between grid resolution and cloud turbulence scales in standard NWP.

Forecasting techniques based on cloud motion will benefit from enhancements in cloud detection approaches for both satellite-based and sky-imager-based methods. Improved update cycles of satellite imagery (e.g., 5-minute updates in the United States through Geostationary Operational Environmental Satellite-R Series [GOES-R]) will provide some information that could so far only be derived from sky imagers for very short-term forecasting. It has become increasingly clear that accurate physics-based forecasting with sky imagers requires 3D reconstruction of the cloud field (Kurtz et al. forthcoming), and further research in this area is required.

With respect to statistical methods, apart from model development, the availability of highquality and up-to-date measurement data of solar irradiance and solar power will be of critical importance. Increasingly more powerful Artificial Intelligence techniques are being developed, which should lead to substantial progress. Finally, an optimized combination of different physical and statistical models will be an advantage for any solar power prediction system.

\footnotetext{
${ }^{1}$ See for an example in the United States: https://ruc.noaa.gov/.
} 


\subsubsection{Probabilistic Solar Forecasting}

Specification of the expected uncertainty of solar irradiance or power predictions for different weather situations provides valuable additional information to forecast users and serves as a decision aid. Therefore, users are increasingly demanding that probabilistic forecasts replace traditional deterministic ones. An advantage of probabilistic forecasts is that forecast uncertainty can also be quantified. Probabilistic forecasting may be based on the use of NWP ensemble prediction systems as well as on the statistical analysis of the distributions of historic predictions and measurements. As in deterministic forecasting, ultimately the combination of physical and statistical methods will lead to the best results.

\subsubsection{Solar Forecast Evaluation}

A proper accuracy assessment is essential for further model development as well as a basis for decision making for users who rely on forecasts. The work performed in the IEA Solar Heating and Cooling Programme's (SHC's) Task 36 and 46 to establish a transparent framework for forecast evaluation is currently continued and extended in the IEA PVPS Task 16. This includes the investigation of new evaluation measures and concepts - for example, to assess variability information about different spatial and temporal scales as well as NWP and cloud motion vector (CMV) model intercomparison studies for different climatic regions.

\subsection{High-Resolution Spatio-Temporal Data}

Most solar resource applications for solar energy systems require long-term data but not necessarily high temporal or spatial resolution. However, there are several applications where solar resource data at high spatial resolution and especially high temporal resolution are beneficial. An example is the electrical generation by concentrating solar thermal electric power plants due to their thermal lags. Calculation of self-consumption shares of PV ("behind the meter") needs high temporal resolution because the variability of the solar resource on buildings and on their load is very high.

Aspects of variability will be investigated with the goal to make recommendations on the number of stations in a power plant or region and on the use of other sensors for this purpose, such as allsky imagers.

Historical long-term solar resource data are typically available for hourly time intervals. More sub-hourly time-series data (ideally 1 minute) with dense spatial coverage (e.g., $1 \mathrm{~km}$ or less) are needed. Higher resolution continental-scale databases are starting to become available (e.g., Monitoring Atmospheric Composition and Climate - Radiation [MACC-RAD], as described in Chapters 4 and 5 , or various commercial products). ${ }^{2}$

For the creation of consistent long-term irradiance data sets, reanalysis models offer a lot of potential. Future work should also be related to cloud assimilation methods and cloud physics because their current weakness is to underestimate clouds and overestimate surface irradiance.

With satellite update cycles limited to 15 minutes over most regions of the globe, 1-minute data have to be derived through modeling. A few generic models that provide high-resolution spatio-

\footnotetext{
${ }^{2}$ For example, https://www.solaranywhere.com/validation/data/resolution/ provides 1-km, 1-minute data and http://solargis.com/products/time-series-and-tmy-data/overview/ provides 250-m, 10-minute data.
} 
temporal data have emerged (Yang et al. 2014; Bright et al. 2017; Hofmann et al. 2014; Munkhammar et al. 2017; Ngoko et al. 2014), but further improvements and validation are needed. IEA PVPS Task 16 will seek to generate further progress in this area.

Photodiode sensors and data acquisition equipment can easily acquire solar irradiance data in intervals as often as 1 second (Wilcox and Myers 2008). Research is underway to deploy solar resource measurement stations that will provide high-resolution data at single locations and within the collector fields. Regarding radiation measurement, future work will also be related to recommendations on meteorological stations, including their operation and the selection of measurement devices depending on the site, the development stages of the solar project, and its size in terms of power.

Further improvement of solar radiometers to avoid soiling of instruments (especially pyrheliometers) should be made. Pyranometers would benefit from a decrease in both cosine errors and lower thermal offsets. All thermopile radiometers would benefit from faster sensors. The uncertainty of the radiation data is another topic in itself. Detailed analysis of the uncertainty of all involved resource products and the combined final products will need to be carried out by various agencies.

Because the maintenance of meteorological ground stations is often a fundamental problem for collecting high-quality data, instruments that require less maintenance than common pyranometers and pyrheliometers with thermal sensors are of interest. Such sensors and their calibration and application must be standardized to increase their applicability to solar resource assessments. In that regard, more versatile and capable all-sky imagers could also lead to the development of a multipurpose single instrument to simultaneously obtain solar irradiance, AOD, thin and thick cloud fractions, and short-term forecasts.

Future work will be necessary in the field of quality control and gap-filling. Standards for adequate irradiance control filters and gap-filling need to be developed. A standard method for the detection of soiling and its correction should also be developed.

\subsection{Additional Measurands}

Although direct normal irradiance (DNI), global horizontal irradiance (GHI), or global tilted irradiance (GTI) are the most important meteorological input parameters for the prediction of the solar power plant yield, several additional parameters must also be provided at high resolution for accurate yield analysis. One important parameter is wind speed due to its spatial variability and effect on panel temperature and concentrating solar power (CSP) mirror stability. Other solar energy-specific measurands include ambient temperature, soiling rates of plant components, the sunshape/circumsolar radiation, and the extinction of radiation between the mirror and the receivers (Hanrieder et al. 2017). The latter two could be modeled from AOD if it could become a common measurand, using, for example, inexpensive sunphotometers. Often, no site-specific information on these properties is available, which results in remarkable impacts on the accuracy of the yield prediction. Hence, these parameters have to be studied in more detail in the future. This topic will be investigated under Subtask 1 of the IEA PVPS Task 16. 


\subsection{Effects of Climate Change on Solar Resource Assessments}

Changes in atmospheric aerosol loading from natural causes or industrial pollution, changing patterns of precipitation and cloudiness, temperature extremes, and other climatic variables may have recently affected the solar resource and may affect its future even further (Huber et al. 2016). This could be important when estimating the performance of a solar power plant throughout the system design life (e.g., 25 years). Research is needed to advance climate modeling capabilities and merge the outputs of these models with advanced system performance models. The identification and treatment of years with impact of exceptionally strong volcanic eruptions should be standardized because they impact the probabilities of exceedance, which are a key factor in the bankability of large solar systems. Further investigation is needed, including liaising with the volcanology scientific community. Subtask 2 in the IEA PVPS Task 16 will seed further progress in this area.

\subsection{Advanced Creation of Typical Meteorological Years (TMY) and Exceedance Values}

Another field of future work is the creation of more appropriate TMY data sets, including the years corresponding to exceedance probabilities other from 50\% (e.g., 90\%, 95\%, and 10\%). The methods should consider various approaches (e.g., Monte Carlo simulation) and realistic statistical distributions of the yearly irradiation. Furthermore, the methods to determine the accuracy of the exceedance values should be improved. 


\section{References}

Bright, J.M., O. Babacan, J. Kleissl, P. G. Taylor, and R. Crook. 2017. "A Synthetic, Spatially Decorrelating Solar Irradiance Generator and Application to a LV Grid Model With High PV Penetration." Solar Energy 147: 83-98.

Hanrieder, N., S. Wilbert, D. Mancera-Guevara, R. Buck, S. Giuliano, and R. Pitz-Paal. 2017. "Atmospheric Extinction in Solar Tower Plants-A Review." Solar Energy 152, 193-207, ISSN 0038-092X, http://dx.doi.org/10.1016/j.solener.2017.01.013.

Hofmann, M., S. Riechelmann, C. Crisosto, R. Mubarak, and G. Seckmeyer. 2014. "Improved Synthesis of Global Irradiance with One-Minute Resolution for PV System Simulations." International Journal of Photoenergy. 2014, Article ID 808509. doi:10.1155/2014/808509.

Huber, I., L. Bugliaro, M. Ponater, H. Garny, C. Emde, and B. Mayer. 2016. "Do Climate Models Project Changes in Solar Resources?" Solar Energy 129: 65-84.

Kurtz, B., F. A. Mejia, and J. Kleissl. Forthcoming. "A Virtual Sky Imager Testbed for Solar Energy Forecasting." Solar Energy accepted with revisions.

Munkhammar, J., J. Widén, and L. M. Hinkelman.. 2017. “A Copula Method for Simulating Correlated Instantaneous Solar Irradiance in Spatial Networks.” Solar Energy 143: 10-21.

Ngoko, B. O., H. Sugihara, and T. Funaki. 2014. "Synthetic Generation of High Temporal Resolution Solar Radiation Data Using Markov Models.” Solar Energy 103: 160-70.

Schalkwijk, J., H. J. Jonker, A. P. Siebesma, and E. Van Meijgaard. 2015. "Weather Forecasting Using GPU-Based Large-Eddy Simulations.” Bulletin of the American Meteorological Society 96(5): 715-23.

Wilcox, S. M., and D. Myers. 2008. Evaluation of Radiometers in Full-Time Use at the National Renewable Energy Laboratory Solar Radiation Research Laboratory. NREL/TP-550-44627.

Golden, CO: National Renewable Energy Laboratory. http://www.nrel.gov/docs/fy09osti/44627.pdf.

Yang, D., Z. Dong, T. Reindl, P. Jirutitijaroen, and W. M. Walsh. 2014. "Solar Irradiance Forecasting Using Spatio-Temporal Empirical Kriging and Vector Autoregressive Models With Parameter Shrinkage." Solar Energy 103(0): 550-62 